\title{
Infrared and Ultraviolet Star Formation in Brightest Cluster Galaxies in the ACCEPT Sample
}

\author{
Aaron S. Hoffer \\ Michigan State University, Physics 83 Astronomy Dept., East Lansing, MI 48824-2320 \\ hofferaa@msu.edu \\ Megan Donahue \\ Michigan State University, Physics 83 Astronomy Dept., East Lansing, MI 48824-2320 \\ donahue@pa.msu.edu \\ Amalia Hicks \\ Michigan State University, Physics 83 Astronomy Dept., East Lansing, MI 48824-2320 \\ hicksam@msu.edu \\ R.S. Barthelemy \\ Western Michigan University, Physics Dept., Kalamazoo, MI 49008-5252 \\ ramon.s.barthelemy@wmich.edu
}

\begin{abstract}
We present infrared (IR) and ultraviolet (UV) photometry for a sample of brightest cluster galaxies (BCGs). The BCGs are from a heterogeneous but uniformly characterized sample, the Archive of Chandra Cluster Entropy Profile Tables (ACCEPT), of X-ray galaxy clusters from the Chandra X-ray telescope archive with published gas temperature, density, and entropy profiles. We use archival Galaxy Evolution Explorer (GALEX), Spitzer Space Telescope, and Two Micron All Sky Survey (2MASS) observations to assemble spectral energy distributions (SEDs) and colors for BCGs. We find that while the SEDs of some BCGs follow the expectation of red, dust-free old stellar populations, many exhibit signatures of recent star formation in the form of excess UV or mid-IR emission, or both. We establish a mean near-UV (NUV) to $2 M A S S \mathrm{~K}$ color of $6.59 \pm 0.34$ for quiescent BCGs. We use this mean color to quantify the UV excess associated with star formation in the active BCGs. We use both fits to a template of an evolved stellar population and library of starburst models and mid-IR star formation relations to estimate the obscured star formation rates. We show that many of the BCGs in X-ray clusters with low central gas entropy exhibit enhanced UV (38\%) and
\end{abstract}


mid-IR emission (43\%) from 8-160 microns, above that expected from an old stellar population. These excesses are consistent with on-going star formation activity in the BCG, star formation that appears to be enabled by the presence of high density, X-ray emitting intergalactic gas in the the core of the cluster of galaxies. This hot, X-ray emitting gas may provide the enhanced ambient pressure and some of the fuel to trigger the star formation. This result is consistent with previous works that showed that BCGs in clusters with low central gas entropies host $\mathrm{H} \alpha$ emission-line nebulae and radio sources, while clusters with high central gas entropy exhibit none of these features. GALEX UV and Spitzer mid-IR measurements combined provide a complete picture of unobscured and obscured star formation occurring in these systems. We present IR and UV photometry and estimated equivalent continuous star formation rates for a sample of brightest cluster galaxies.

Subject headings: galaxies: elliptical and lenticular, $\mathrm{cD}$

\section{Introduction}

The basic story underlying our current models for the formation of galaxies and clusters of galaxies is that baryonic matter falls into dark matter potential wells, cools to make cold molecular clouds, which then form stars and supermassive black holes. The state of the gas as it falls, the morphology of the accretion, the source of the dust that catalyzes formation of molecular clouds, the physical processes determining the gas temperatures and phases are all uncertain. Simply put, we do not know the full story of how intergalactic gas eventually forms stars and black holes.

Brightest Cluster Galaxies (BCGs) provide unique opportunities for the investigation of the role of hot intergalactic gas in galaxy formation, and in particular its role in affecting the evolution of the star formation and active galactic nucleus (AGN) activity in the central galaxy in the most massive dark matter halos in the universe. The intergalactic gas bound to a massive cluster of galaxies - its intracluster medium (ICM) - outweighs the stars in those galaxies by a factor of 5-10 (e.g., David et al. 1990; Arnaud et al. 1992; Gonzalez et al. 2007). The BCGs in the centers of $\mathrm{X}$-ray clusters where the gas has a short cooling time (or equivalently, low gas entropy) exhibit signs of activity (e.g. radio sources, emission-line nebulae, excess blue or ultraviolet light) that are rare in BCGs in other clusters of galaxies (Hu et al. 1985; Burns 1990; Cavagnolo et al. 2008; Rafferty et al. 2008; Sanderson et al. 2009; Sun 2009). The activity in the BCGs of this category of clusters has been presented as evidence that hot ICM condenses into cold dusty gas that subsequently forms stars. Such BCGs may be hosting real-life versions of late-time $(z<1)$ accretion onto supermassive black holes in central galaxies; but the role of the hot ICM in AGN or star formation activity is not entirely clear.

The simplest hypothesis for how hot gas cools when it is confined to a massive dark halo fails. The first X-ray observations of the ICM in galaxy clusters indicated that some clusters have 
a high central gas densities and central cooling times shorter than the age of the universe(e.g., Fabian \& Nulsen (1977); Cowie \& Binney (1977)). In this scenario, such gas cools slowly, loses pressure support, compresses, allowing gas from the outer parts of the cluster to settle gently into the center. The inferred mass accretion rates could be as large as $1000 \mathrm{M}_{\odot} \mathrm{yr}^{-1}$ (Fabian 1994). Such clusters were dubbed "cooling flows." However, higher resolution X-ray spectroscopy showed that the luminous emission lines one would expect from gas cooling smoothly from $10^{8} \mathrm{~K}$ to non-Xray emitting temperatures were not present (Peterson et al. 2003). Nevertheless, such clusters do exhibit cool cores with radii $\sim 50-100 \mathrm{kpc}$, where $k T_{\text {core }} \sim 1 / 2-1 / 3$ of that found in the outer radii. These clusters are now often called "cool core" clusters.

With spatially resolved X-ray spectroscopy, cool core clusters can be classified by the distribution of gas entropy of the galaxy cluster. The cluster entropy is a thermodynamic quantity. Conveniently, in a gas of pure hydrogen emitting thermal bremsstrahlung radiation, the cooling time can be written down solely in terms of the gas entropy. The gas entropy $S$ is proportional to the logarithm of the quanitity $K=\mathrm{T}_{\mathrm{X}} \mathrm{n}_{\mathrm{e}}^{-2 / 3}$, conventionally reported in units of $\mathrm{keV} \mathrm{cm}^{2}$. Donahue et al. (2006) radially fit entropy profiles with a functional form $\mathrm{K}(\mathrm{r})=\mathrm{K}_{0}+\mathrm{K}_{\mathrm{x}}\left(\mathrm{r} / \mathrm{r}_{\mathrm{x}}\right)^{\alpha}$, where $K_{0}$ is the central entropy in excess above the power law fit. Cavagnolo et al. (2008) extended this procedure to the entire Chandra archive, creating the Archive of Chandra Cluster Entropy Profile Tables 1 (ACCEPT). Galaxy clusters with high central entropy often contain quiescent brightest cluster galaxies (BCGs) or exhibit evidence for significant merger or interactions. The empirical boundary between clusters which occasionally host active BCGs and clusters which never host them is $\mathrm{K}_{0} \sim 30 \mathrm{keV} \mathrm{cm}^{2}$, an entropy associated with an ICM cooling time of $\sim 1$ Gyr (Voit et al. 2008). Furthermore, about $70 \%$ of the BCGs in those cool core clusters host radio sources, and about half of those host extended emission-line nebula characteristic of lowionization nuclear emission-line regions (LINERs; but are more extended) (Heckman et al. 1989; Crawford et al. 1999; Donahue et al. 2010). Cavagnolo et al. (2008) and Rafferty et al. (2008) have shown that only those BCGs inhabiting clusters with low central gas entropies (short central gas cooling times, high central gas densities) present low-ionization emission-line nebulae ( $\mathrm{H} \alpha$ ), blue gradients, or radio sources.

In this paper, we look for signatures strongly associated with star formation, ultraviolet (UV) excesses and mid-infrared (mid-IR) emission from dust, in the ACCEPT sample of well-studied Xray clusters. Since even an evolved stellar population emits some UV (and mid-IR), we characterize the stellar content of the BCG using Two Micron All Sky Survey (2MASS) K-band photometry and photometry from the IRAC instrument on the Spitzer Space Telescope, short-wavelength 3.6 and 4.5 micron bands, where available. To estimate the contribution of recent star formation we measure the ultraviolet (UV) emission with the Galaxy Evolution Explorer (GALEX) observations. The UV samples the peak of emission in short-lived $\mathrm{O}$ and B stars, thus tracking recent, unobscured star formation. Most of the star formation in the universe occurs hidden within cold, dusty

\footnotetext{
${ }^{1}$ http://www.pa.msu.edu/astro/MC2/accept/
} 
molecular clouds. The dust in these clouds absorbs the UV and optical light of buried stars and re-emits this light as mid-IR thermal radiation typical of dust at $\sim 100 \mathrm{~K}$. Some of this reprocessed emission emerges in the form of features, such as the emission complexes associated with polycyclic aromatic hydrocarbons (PAHs) (Donahue et al. 2011). Puzzlingly, powerful $\mathrm{H}_{2}$ features appear to be nearly ubiquitous in systems with $\mathrm{H} \alpha$ nebulae, at levels unlikely to be associated with typical star formation processes (Elston \& Maloney 1994; Jaffe \& Bremer 1997; Donahue et al. 2000, 2011; Egami et al. 2006). Even colder dust (20-30K) in the far-IR has been seen with Herschel (Edge et al. 2010a) (Edge et al. 2010b), and Edge (2001) detected significant masses of CO.

The measurements of star formation in BCGs based on UV or mid-IR information to date have been relatively limited. For example, Catinella et al. (2010) report that star formation efficiency varies little over a wide range of galaxy masses in a massive galaxy sample. However, while that sample includes 190 massive galaxies observed with GALEX and Arecibo, it has very few BCGs. Donahue et al. (2010) assessed the UV properties of the BCGs in a representative sample of $30 \mathrm{X}$ ray selected clusters from the Representative XMM-Newton Cluster Structure Survey (REXCESS) (Böhringer et al. 2007), while most UV studies are of a limited set of the most extreme emission-line BCGs (e.g., Hicks et al. 2010; O'Dea et al. 2010). Quillen et al. (2008) and O'Dea et al. (2008) studied 62 BCGs with Spitzer, selected for their luminous $\mathrm{H} \alpha$. To expand upon these studies, we present an assessment of the UV, near-IR and mid-IR properties of BCGs in a well-studied sample of X-ray clusters. This sample is larger and more diverse than previous studies, as it includes quiescent BCGs along with the most extreme cool-core BCGs. In Section 2 we briefly describe the original X-ray cluster sample, and give an overview of the GALEX and Spitzer observations. We describe how the BCGs are identified. In Section 3 we discuss the data reduction process for the images in the Spitzer, GALEX, and 2MASS archives. Our discussion and analysis of the data is in Section 4. We present estimates of the equivalent continuous UV and IR star formation rates in this section. UV colors are compared to those in Wang et al. (2010). We present a summary of the observations, detections, and emission excesses in Table 1. We conclude the paper in Section 5. For all calculations the assumed cosmology is $H_{0}=70 \mathrm{~km} \mathrm{~s}^{-1} \mathrm{Mpc}^{-1}, \Omega_{M}=0.3, \Omega_{\Lambda}=0.7$.

\section{Observations}

\subsection{Chandra X-Ray Observations}

The original galaxy cluster sample is from the ACCEPT database (Cavagnolo et al. 2009), which includes 239 galaxy clusters. This sample is a selection of all galaxy clusters in the Chandra archive as of August 2008 that met a minimum flux criterion. The clusters were selected to construct entropy profiles and provide central entropy estimates. To be able to accurately measure the entropy profiles, temperature gradients were required to have a precision better than $\Delta \mathrm{kT}_{\mathrm{X}} \approx \pm 1.0 \mathrm{keV}$. Cavagnolo et al. (2009) therefore required at least three concentric annuli with a minimum of 2500 counts each. The search resulted in 317 observations of 239 galaxy clusters. Six groups from the flux- 
limited Highest X-ray Flux Galaxy Cluster Sample (HIFLUGCS) sample (Reiprich \& Böhringer 2002) were added to the collection and a number of clusters with analysis complications were removed. (All additional objects are listed in Cavagnolo et al. (2009).) This sample is not a formally complete sample, but, by and large, these clusters were not selected to be included in the Chandra program because of the UV and mid-IR properties of their brightest cluster galaxies. An interestingly large fraction of these clusters now have been observed by GALEX and Spitzer, and so the time is right for a uniform analysis of the X-ray, UV, and mid-IR properties of the BCGs in the sample.

\subsection{MASS Observations - BCG identification}

We used the 2MASS archive and previous literature to determine the locations of the the BCGs in these galaxy clusters (Table 2). The brightest cluster galaxies were initially identified by their $2 M A S S$ position. The locations of the BCGs were determined using a visual inspection (including source brightness and morphology) with $2 M A S S$ J-band images $5^{\prime} \times 5^{\prime}$ in size centered on the X-ray centroid to determine the brightest galaxy in the cluster. This visual inspection was followed up with NASA/IPAC Extragalactic Database (NED)2 and the Set of Identifications, Measurements, and Bibliography for Astronomical Data (SIMBAD) 3 object searches within $2^{\prime}$ of the X-ray centroid to verify the redshifts of the candidate BCGs. All objects in the 2MASS Extended Source Catalog (Jarrett et al. 2003) were checked for redshift information and any other indication that they are the brightest galaxy in the cluster. Some BCGs were too distant to have associated 2MASS catalog entries. The BCGs of these distant clusters were identified using a literature search for journal articles indicating the location of the BCG in the cluster, and are named by their right ascension and declination. For the clusters in the Sloan Digital Sky Survey (SDSS) footprint, color information and brightness in the optical (u'g'r'i'z') from the data release 7 (DR7) were used to verify the BCG selections (Leisman et al. 2011). In a small number of cases we revised the original selection of $2 M A S S$ location (Abell 2034, RXJ1022.1+3830,4C+55.16, Abell 2069, Abell 368, and Abell 2255). Table 2 gives each cluster and the $2 M A S S$ coordinate for the brightest cluster galaxy.

In Table 3 we list the physical separation of the BCGs from the X-ray centroid of their host galaxy clusters. While most BCGs lie near the X-ray centroid of their galaxy cluster, consistent with their identification as $\mathrm{cD}$ galaxies, there are a few that are very far from the center. The physical distance between the X-ray centroid and the BCG is plotted as a histogram in Figure 1 . The BCG we identified is twice as likely to be within 10 projected kpc of its X-ray centroid in low entropy clusters $(74 \%)$ compared to high entropy clusters $(37 \%)$. Note that all BCGs in a low $\mathrm{K}_{0}$ system are within $40 \mathrm{kpc}$ of their cluster's X-ray centroid.

${ }^{2}$ http://nedwww.ipac.caltech.edu/

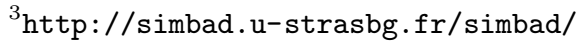




\subsection{GALEX Observations}

The Galaxy Evolution Explorer $(G A L E X)$ obtains images in the near UV (NUV) at $\lambda_{e f f}=$ $2267 \AA$ (bandpass with a full width at half maximun (FWHM) of $269 \AA$ ) and the far UV (FUV) at $\lambda_{\text {eff }}=1516 \AA$ (FWHM of $616 \AA$ ) (Martin et al. 2005). There are a total of 168 BCGs in our initial sample with GALEX observations in the GALEX archive as of 2011 October. We then searched the GALEX Release Six (GR6) catalog for a UV source within $5^{\prime \prime}$ of the 2MASS BCG location. In the cases where there were multiple observations, the observation with the highest signal to noise was used. Table 2 gives the GALEX object identifiers for each BCG detected. Note that not all observations will have an object identifier as the BCG may have gone undetected in the GALEX archive.

\subsection{Spitzer Observations}

We analyzed archival Spitzer Infrared Array Camera (IRAC) and the Multiband Imaging Photometer for SIRTF (MIPS) observations. IRAC has four near infrared wavebands at 3.6, 4.5, 5.8, and $8.0 \mu \mathrm{m}$ (Fazio et al. 2004). MIPS (Rieke et al. 2004) operates in the mid-IR and has three wavebands at 24, 70, and $160 \mu \mathrm{m}$. The Spitzer imaging observations selected for analysis were aimed within $1^{\prime}$ from the X-ray centroid. The Astronomical Observing Request (AOR) numbers are given in Table 2. There are 79 brightest cluster galaxies in ACCEPT with IRAC observations and 100 ACCEPT BCGs with MIPS observations as of 2010 December.

\section{Aperture Photometry and Colors}

\subsection{GALEX UV Photometry}

We used GALEX aperture photometry provided in the GALEX catalog and GALEXView 4 . We chose apertures to match GALEX measurements and derive colors with photometry from other catalogs (e.g. 2MASS, SDSS) and with our Spitzer aperture photometry. The optimal aperture for the UV is determined by comparing the estimate of the total flux given in GALEXView to the circular aperture flux. The circular aperture chosen is the one with the flux measurement nearest to the estimated total flux value. For most BCGs the two largest aperture radii $\left(12.8^{\prime \prime}\right.$ and $17.3^{\prime \prime}$ ) were used. The minimum allowed aperture radius was 9.0" to avoid aperture correction (the FWHM of GALEX observations are $\sim 4.5^{\prime \prime}-6^{\prime \prime}$ ). The GALEX-detected UV emission is usually centrally concentrated so generally, the UV emission lies within a radius of $9^{\prime \prime}$ even when the angular size, as seen in the optical, of the galaxy is larger. Therefore, the GALEX aperture size is an approximate upper limit on the size of the UV star formation region. Some of the UV

${ }^{4}$ http://galex.stsci.edu/GalexView/ 
light is produced by evolved stars (e.g., O'Connell 1999) so we use the NUV-K color to estimate how much UV comes from recent star formation. We make photometric measurements within sufficiently large apertures to minimize the degree to which aperture corrections could affect our conclusions. The magnitudes are converted from the magnitudes given in the GALEX catalog to AB magnitudes using zeropoints of 20.08 magnitudes for the NUV and 18.82 magnitudes for the FUV (Morrissey et al. 2007). The Galactic extinction corrections are applied from Schlegel et al. (1998) assuming a ratio of 3.1 for $A_{V} / E_{B}-\mathrm{V}$. The NUV correction assumed is $3.25 A_{V}$ and the FUV correction is $2.5 \mathrm{~A}_{\mathrm{V}}$. UV photometry is presented in Table 4.

\section{2. $\quad G A L E X$ UV Upper Limits}

To estimate the detection threshold for $G A L E X$ observations, we evaluated the cataloged fluxes of all the well-detected sources with a magnitude error $<0.35(S / N \gtrsim 3)$ within $1^{\circ}$ of the BCG targets. Our GALEX upper limits are based on detections of peaked sources, i.e. point sources and compact emission regions. A uniform, extended source that fills the aperture will have a higher detection threshold than this estimate. We plot these fluxes as a function of their individual exposure times in Figure 2. The estimated detection threshold is inferred from the upper envelope of these points, which is approximated here by curves $\propto t^{1 / 2}$. For the exposure times typical of the allsky imaging survey (AIS) the estimate for the upper limit in AB magnitudes is $19+1.25 \times \log t_{N U V}$ for an exposure time $t_{N U V}$ in seconds. Similarly the function for AB magnitude upper limit for the $\mathrm{FUV}$ is $18.5+1.25 \times \log t_{F U V}$. This relation underestimates the $G A L E X$ sensitivity for longer exposure times, longer than $\sim 500$ seconds. There are 9 BCGs (Abell 2319, 3C 295, Abell 611, Abell 665, Abell 1942, Abell 2631, CL J1226.9+3332, HCG 62, and Abell 2219) which had UV exposure times greater than 500 seconds and have a nondetection. For these objects we looked in the field and set the upper limit to be equal to the dimmest source that was detected (with a magnitude error less than 0.35). We report this estimated $3 \sigma$ upper limit for all cases where the BCG was undetected and when the GALEX source flux had a large error (>0.35 mag), indicating a highly uncertain detection. For BCGs with NUV upper limits, the 2MASS fluxes are matched with a $7^{\prime \prime}$ aperture such that they are similar in size to the GALEX PSF.

\subsection{Spitzer Near and Mid IR Photometry}

For the vast majority of the observations, Spitzer photometry was measured from the final pipeline product post-Basic Calibration Data (pbcd). The pipeline data were flux calibrated in units of MJy steradian ${ }^{-1}$ (Reach et al. 2005) from the IRAC pipeline version S18.7.0 and the MIPS pipeline version 16.1.0. For the four IRAC wavebands, fluxes were measured inside a circular aperture with a radius of $r=14.3 \mathrm{kpc} \mathrm{h}_{70}^{-1}$. We wrote an IDL program to perform all aperture flux measurements for Spitzer (Donahue et al. 2010). The circular aperture is centered on the BCG location in Table 2, The backgrounds were computed from an annulus with an inner radius of 
$35^{\prime \prime}$ and an outer radius of $45^{\prime \prime}$ for objects which have an angular radius smaller than $35^{\prime \prime}$. For objects with flux beyond the nominal aperture, the background was computed with an annulus with an inner radius of $1.1 \times$ the radius for the object and an outer radius $1.3 \times$ the radius of the object. To estimate the mean background counts we fit a Gaussian to a histogram of counts per pixel in the background annulus. This procedure provides a background estimate that is robust to possible sources of contamination (e.g. foreground stars) that increase the counts in a small number of background pixels but do not significantly influence the mean of the Gaussian.

Most of the galaxies detected by MIPS are essentially point sources because the FWHM of the point spread function (PSF) for 24,70 , and $160 \mu \mathrm{m}$ are $6^{\prime \prime}, 18^{\prime \prime}$, and $40^{\prime \prime}$, respectively. We measure MIPS fluxes using the same IDL code. Since not all of the flux from the PSF falls in the aperture, MIPS aperture fluxes are corrected using the same aperture correction methods in $\S 4.3 .4$ of the MIPS Handbook 5 . The 24 micron aperture radius is fixed at $13^{\prime \prime}$ with a background annulus of $15-25^{\prime \prime}$ giving a flux correction factor of 1.167. Similarly, the 70 micron aperture radius is fixed at $35^{\prime \prime}$ with a background annulus of 40-60", and a correction factor, assuming a $30 \mathrm{~K}$ source, of 1.22. The 160 micron observations were measured at an aperture radius of $40^{\prime \prime}$ with a background annulus of 64-128", and a correction factor of 1.752 (also assuming a 30K source). We also derived flux estimates using software provided by the Spitzer Science Center, APEX in MOPEX (Makovoz \& Marleau 2005), to cross-check our aperture flux measurements. The standard input parameters were used and residual images were created to assess whether the source was completely subtracted. For all sources with proper subtraction, the flux measurement from APEX was compared to the aperture measurement and we verified they were consistent within the cited errors. Only the flux values calculated from apertures are included in Table 5. In Table1 detections and excesses are equivalent for the 70 and 160 micron observations as we do not have an a priori belief that quiescent BCGs should exhibit 70 and 160 micron emission.

For the closest and, likely, spatially-extended BCGs, the fluxes from APEX were systematically lower than the aperture flux estimates. To determine whether any BCG had extended emission or contamination from unrelated point sources, we compared aperture-corrected flux measurements with $13^{\prime \prime}$ and $35^{\prime \prime}$ apertures, and we inspected the 24 micron images for point source contamination within the $35^{\prime \prime}$ radius aperture. Visible contamination was classified as either insignificant, because the difference between the two aperture-corrected estimates was smaller than the statistical uncertainty of those fluxes, or significant. We inspected all detections for possible contamination inside $35^{\prime \prime}$ but we only found potential contamination in the annulus between the $13^{\prime \prime}$ and the $35^{\prime \prime}$ radii (i.e. we saw no obvious sources of contamination inside $13^{\prime \prime}$ ). Therefore, we do not expect contamination to affect the 24 micron point source flux measurements listed in Table 5 . However, the existence of any contaminating source seen at 24 microns is flagged for our 70 and 160 micron photometry in Table 5, which uses larger apertures. (Refer to the footnotes in Table 5 for a description of the contamination categories.)

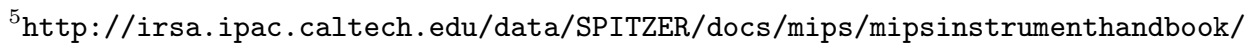


BCGs which did not have point source contamination visible at 24 microns but showed an increase in flux over that expected for a point source in the larger $35^{\prime \prime}$ aperture are considered extended. All the objects which have been identified as such are, unsurprisingly, nearby galaxies. Instead of correcting the fluxes of these objects as if they were point sources at 24 microns, the fluxes for these galaxies are reported for the large apertures we used for the IRAC photometry. (One exception, the BCG NGC 4636, was measured at a $35^{\prime \prime}$ radius instead because of significant point source contamination beyond this aperture.)

To more directly account for 70 micron contamination, if a 70 micron source was listed as a detection and the 24 micron measurement indicated contamination, the 70 micron image was inspected for contaminating sources. If a 70 micron detected source inside the aperture appears to come from an object other than the BCG (i.e. its centroid is consistent with that of a non-BCG galaxy) then the detection was downgraded to a conservative upper limit. However, these 70 micron upper limits are based on photometry using a smaller, $16^{\prime \prime}$ aperture radius with the corresponding point source correction of 1.94 to avoid including flux from extraneous point sources in the upper limit. The 70 micron upper limits estimated through this method are noted in the table. There are two BCGs, Abell 2744a and MS 04516-0305, that are contaminated at 70 microns as well as 160 microns. Upper limits for their 160 micron photometry were found using the same $16^{\prime \prime}$ aperture radius with the corresponding point source correction of 4.697 .

The standard photometric error of $5 \%$ is used for the IRAC points as the systematic errors were always much larger than the statistical errors. For MIPS the standard errors are 10\%, 20\%, $20 \%$ for 24, 70, and 160 microns, respectively. These standard errors are usually good estimates except in the case of lower $S / N$ detections for which statistical uncertanties are important (i.e. $S / N=5-20$ ). We report the total errors (including statistical and systematic uncertainties) for MIPS with the flux measurements in Table 5 .

For MIPS, upper limits were estimated for detections that are below $5 \sigma$. The standard deviation of the observation was calculated in the same manner as Donahue et al. (2010). If the standard aperture flux had a $S / N<5$ the filtered data were used instead. The background on these data are better controlled, but the MIPS Handbook warns that low surface brightness emission in the filtered data will be lost. Therefore, the filtered data were only used when the standard source detection fell below the $5 \sigma$ limit. Those filtered images that are still below the $5 \sigma$ detection threshold were assigned a $5 \sigma$ upper limit for that detection. If a BCG is undetected with the standard mosaic but is detected $(>5 \sigma)$ using the filtered data it is considered a filtered detection and is labelled as such in the Table 5. There were many observations that were considered filtered detections in our first pass through the data, but were revised to upper limits because of 70 micron contamination from non-BCG sources.

For a few of the nearest and brightest BCGs there was an issue with the final data products in the Spitzer pipeline. In these cases, the BCG contained a spurious point source that was much brighter than the rest of the galaxy. These very bright artifacts proved to not be physical because 
the anomalous levels were not detected in the individual BCD frames. We mosaicked the individual BCD frames with the MOPEX software using the standard mosiacking procedure and settings. The new mosaic images did not exhibit the spurious point sources. The fluxes were then calculated from the new images and were in agreement with the original images if the point source was masked out. Those AORs which required remosaicking are noted in Table 2 .

\subsection{MASS Near IR Observations}

2MASS J, $\mathrm{H}$ and $\mathrm{K}$ fluxes and errors are extracted from the 2MASS Extended Object Catalog (Jarrett et al. 2003). The catalog provides aperture photometry between $5^{\prime \prime}$ and $60^{\prime \prime}$ in radius. For a few large galaxies (e.g. M87, M49, NGC 4696) the aperture photometry was taken from the 2MASS Large Galaxy Atlas. The measurements were converted from the system's Vega magnitudes to Janskys using the AB magnitude conversions $(0.9,1.37$, and 1.84 mag for J, H, and K bands, respectively) provided in Cohen et al. (2003). We correct 2MASS magnitudes for Galactic extinction: $A_{K}=0.112 A_{V}, A_{J}=0.276 A_{V}, A_{H}=0.176 A_{V}$ (Schlegel et al. 1998). In order to derive flux ratios normalized to emission dominated by the old stellar population sampled in the near-infrared, we matched apertures in the near-IR with those at other wavelengths. Therefore we estimated $2 M A S S$ photometry (presented in Table 6) for each source in three apertures: (1) the GALEX aperture for $N U V-K,(2)$ the IRAC aperture of $r=14.3 h_{70}^{-1} \mathrm{kpc}$ for IRAC to near-IR flux ratios, and (3) the 24 micron aperture (for K-band only). After extinction and k-correction, Figure 3 shows that

the BCGs have no trend in their $\mathrm{K}$ band luminosity (the mean is $1.6_{-0.4}^{+0.7} \times 10^{44} \mathrm{erg} \mathrm{s}^{-1} \mathrm{~h}_{70}^{-2}$ ) as a function of redshift or $K_{0}$ of these galaxy clusters.

\section{Discussion}

\subsection{UV Excess and Color}

The UV excess is determined by comparing $N U V-K$ colors, plotted in Figure 4 against excess entropy $K_{0}$ from Cavagnolo et al. (2009). The baseline for quiescent BCGs is visible in this figure. The BCGs with excess UV emission, over and above the UV found in quiescent BCGS, are only in the low $\mathrm{K}_{0}$ galaxy clusters in the ACCEPT sample. While we find no BCGs with excess UV emission in galaxy clusters with high central entropy, there are many quiescent BCGs in low central entropy clusters. From our sample we estimate the typical $N U V-K$ color for quiescent BCGs from the mean and standard deviation of all BCGs with central entropies above $30 \mathrm{keV} \mathrm{cm}{ }^{2}$. We derive a mean color of inert BCGs is $6.59 \pm 0.34$. In contrast, the mean color for BCGs in clusters with central entropies less than $30 \mathrm{keV} \mathrm{cm}^{2}$ is $6.11 \pm 0.99$. We define a color excess $\Delta_{\mathrm{c}}=6.59-(\mathrm{NUV}-\mathrm{K})$. This excess will be used in $\S 4.3$ to estimate the equivalent continuous star formation rate. The color excess is simply defined such that blue light in excess of quiescent BCGs in high entropy clusters can easily be translated into a UV luminosity associated 
with continuous unobscured star formation. BCGs are considered to have a NUV excess in Table 1 if their NUV-K color is at least $1 \sigma$ bluer than the mean color of inert BCGs. We see that $38 \%$ of low central entropy clusters in our sample have a NUV-K excess. The BCGs with the bluest colors are in Abell 426, Abell 1664, and RX J1504.1-0248 which have colors around 3.0.

We plot the $F U V-N U V$ and $N U V-K$ colors for BCGs in Figure 5. Contamination from line emission from Ly $\alpha$ may occur if the redshifted Ly $\alpha$ line is included in the FUV bandpass (within

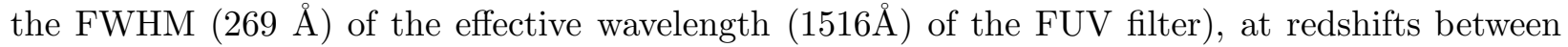
$0.15-0.36$. The right figure plots only nearby BCGs $(\mathrm{z}<0.15)$ to address this possible effect. Excluding the BCGs which may be contaminated by line emission ( $\mathrm{z}>0.15$ ), we do not detect a significant FUV-NUV color difference between bluer BCGs (with NUV-K colors less than 6.3) and redder BCGs (with NUV-K colors greater than 6.3). The mean of the FUV-NUV color for bluer BCGs is $0.73 \pm 0.57$ while the mean of redder BCGs is $0.79 \pm 0.30$.

Wang et al. (2010) uses GALEX and SDSS to measure colors on a sample of 113 nearby $(\mathrm{z}<0.1)$ optically selected BCGs and compare them to a sample of field galaxies. Also, they compare their results to a sample of 21 X-ray selected BCGs from Rafferty et al. (2008) which included BCGs in both cool-core and non-cool-core clusters. From Figure 7 in Wang et al. (2010), the distribution of the FUV-NUV color is consistent with ours with a mean that better matches the photometry from their outer apertures (radius covers $90 \%$ of the light) than that measured within their inner apertures (radius covers $50 \%$ of the light). Similarly, their NUV-r colors are consistent with our NUV-K colors, after transformation between SDSS r and 2MASS K bands, assuming those bands are only affected by emission from the old stellar population.

\subsection{IR Color}

The ratios of 8.0 to 3.6 micron fluxes track the ratios of infrared emission from polycyclic aromatic hydrocarbons (PAHs), stochastically heated hot dust grains, and possibly rotationally excited molecular hydrogen and other emission lines (e.g., Donahue et al. 2011) to emission from stars. We plot these ratios as a function of redshift in Figure 6. The line shows the expectation for a passively evolving stellar population with an age of $10 \mathrm{Gyr}$ at $z=0$. After normalizing the ratio for the stellar population, we determine the total number that are at least $1 \sigma$ above the normalized mean for BCGs in high $K_{0}$ clusters $(1.014 \pm 0.061)$ and refer to those as BCGs with excess 8.0 micron emission in Table 1. The points that lie well above this line are likely to have some form of hot dust and/or PAH emission as the observed IRAC 8.0 micron color is sensitive to only strong PAH features. In Figure 7 the IRAC ratio of 4.5 to 3.6 micron fluxes from Spitzer are plotted against redshift, similarly to the plot from Quillen et al. (2008). Similar to what we have done for the 8.0 to 3.6 micron ratio, we normalize the 4.5 to 3.6 micron ratio for a passively evolving stellar population with an age of $10 \mathrm{Gyr}$ at $z=0$. We then determine a mean of the normalized ratio for BCGs in high $K_{0}$ clusters $(1.048 \pm 0.019)$. All BCGs at least $1 \sigma$ in excess of the mean are considered to have excess 4.5 micron emission. 
For both the 8.0 to 3.6 micron flux ratio and the 4.5 to 3.6 micron flux ratio, the only BCGs with excesses over and above a passively evolving old stellar population are those that inhabit clusters with low central entropies, as shown in Figure 7 and Figure 6. In Figure 8 the 8.0 to 3.6 micron ratio and the 4.5 to 3.6 micron ratio are strongly correlated $(r=0.92,15 \sigma$ for objects with mid-IR detections and/or NUV-K excesses), which is expected if the excess 4.5 micron emission is generated by processes related to that producing the 8.0 micron emission. The functional fit plotted is

$$
\log _{10}\left(\mathrm{~F}_{8.0 \mu \mathrm{m}} / \mathrm{F}_{3.6 \mu \mathrm{m}}\right)=(0.153 \pm 0.002)+(5.422 \pm 0.021) \times \log _{10}\left(\mathrm{~F}_{4.5 \mu \mathrm{m}} / \mathrm{F}_{3.6 \mu \mathrm{m}}\right) .
$$

Both the ratios have been normalized for passive evolution. As long as the IRAC calibration was consistent over time, these are precise relative flux ratios, independent of the flux calibration. The absolute flux ratios are precise to about $2 \%$. The 8.0 and 4.5 micron bandpasses will include PAH and mid-IR emission line features associated with activity seen in cool core BCGs (Donahue et al. 2011). The emission of dust-free, evolved stellar populations in these same bandpasses is similar to the Rayleigh-Jeans tail of a black body, decreasing steeply to longer wavelengths. We see two BCGs HCG 62 and Abell 1644 that show an excess in both normalized ratios however neither shows a NUV-K excess. Abell 1644 was not observed in MIPS and we expect to see a detection in the 70 micron waveband based on this correlation. HCG 62 has a 70 micron upper limit which may be related to the selection effect that it is a very low $K_{0}$ galaxy group.

We assess the presence of a luminous dust component, likely to be obscured star formation but also could be contributed by an AGN, by looking at the 24 micron to K-band (2.2 micron) flux ratio plotted against the central entropy in Figure 9. We note a similar pattern here as found in the UV excess plots (Figure 4), that the low $K_{0}$ galaxy clusters are far more likely to host BCGs with warm dust. The possible exception to this pattern is Abell 521, which is a high entropy cluster with an elevated 24 micron to $\mathrm{K}$ band flux ratio. However, as seen in Ferrari et al. (2006) there is a low entropy, compact, X-ray corona(Sun et al. 2007) (i.e. a "mini-cooling core") around the BCG in Abell 521, embedded in a cluster with otherwise high entropy. Excess 24 micron emission is estimated by determining the mean of the $24-\mathrm{K}$ ratio of BCGs in high $K_{0}$ systems (excluding Abell 521) and any BCG with at least $1 \sigma$ above this mean $(0.063 \pm 0.050)$ is considered to have excess 24 micron emission. We see that $43 \%$ of the cool cores in our sample have an excess in their 24 micron to $\mathrm{K}$ band ratio. The BCGs with the most extreme 24 micron to $\mathrm{K}$ band ratios are in $\mathrm{ZwCl} 0857.9+2107$ and Cygnus A with a ratio of about 20. BCGs in Abell 426 and Abell 1068 also have large ratios around 10. All four objects likely have some AGN contribution. We see the scatter (i.e. standard deviation) in the ratio $\log _{10}\left(\mathrm{~F}_{24 \mu \mathrm{m}} / \mathrm{F}_{\mathrm{K}}\right)$ is 0.81 for BCGs in low central entropy clusters.

We can compare IR ratios in BCGs to those of normal star-forming galaxies and starbursts, similar to Figure 1 in Johnson et al. (2007a). The ratios for the BCGs are plotted in Figure 10 as well as the SINGS galaxies (Kennicutt et al. 2003). Similar to their sample of a wide range of galaxies, the BCGs in our sample have the same colors as star-forming galaxies in SINGS. We note that some of the nearby BCGs have a higher ratio of 8 micron to 24 micron emission by a 
factor of 2. This ratio may indicate a relatively larger contribution from PAH emission over very warm dust. Also, this bandpass may include contributions from the S(4) transition of molecular hydrogen. Rotationally excited molecular hydrogen lines are extremely luminous in some BCGs, and these same lines are not bright in star forming galaxies (Donahue et al. 2011). It is possible that some of the excess emission at the 8.0 micron may be contributed by molecular hydrogen.

\subsection{Star Formation Rates (SFRs)}

The UV color excess, $\Delta_{c}$ defined in $\S 4.1$, can be used to estimate the excess UV luminosity due to unobscured star formation:

$$
\mathrm{L}_{\mathrm{SFR}}=\mathrm{L}_{\nu}\left(1-10^{-\Delta_{\mathrm{c}} / 2.5}\right)
$$

where the specfic luminosity $\mathrm{L}_{\nu}$ is converted from the NUV AB magnitude, corrected for Galactic extinction. The NUV k-correction for a star forming spectrum is negligible out to moderate redshifts (Hicks et al. 2010). The unobscured star formation rate is then estimated from the relation in Kennicutt (1998) and listed in Table 7. The total UV luminosity is estimated to be $\mathrm{L}_{\mathrm{UV}} \sim \nu \mathrm{L}_{\nu}$ using $\nu=\mathrm{c} / 2267 \AA$. Upper limits are based on $3 \sigma$ uncertainties in UV excesses.

The obscured star formation rate is estimated in two ways, (1) by fitting Groves et al. (2008) starburst models and a model of an old stellar population to the 2MASS and Spitzer IRAC/MIPS infrared data points, and (2) from using calibrated conversions of IR luminosity (mostly 24 and 70 micron luminosities) to SFRs. In the first case, we present a sum of the two models, with independent normalizations. Star formation rates were determined for all BCGs with data from at least $2 M A S S$ and the $24 \mu \mathrm{m}$ band of MIPS. To estimate rest-frame IR luminosities based on the 24 and 70 micron fluxes, k-corrections were applied such that $\mathrm{L}_{\mathrm{rest}}=\mathrm{kL}_{\mathrm{obs}}$. The corrections were found using the best-fit Groves model for that individual galaxy and convolving it with the MIPS bandpass, both in the rest frame and the observed frame of the galaxy. The actual 70 micron corrections do not depend very much on the specific Groves starburst model. However, the 24 micron point usually falls around a minimum in the spectrum, which causes a larger scatter in the relation for a give redshift (up to 30\%) The 24 micron k-corrections are in the range (0.125-1.056), the 70 micron k-corrections are in the range (0.738-1.879). The total IR luminosity, $\mathrm{L}_{\text {dust }}$, is estimated by integrating the total scaled starburst model over $\lambda \lambda 8-1000 \mu \mathrm{m}$. We plot the UV star formation rate against the IR star formation rate in Figure 11.

Calibrated conversions for star formation rates from 24 and 70 micron luminosities were used from Calzetti et al. (2010). The 70 micron luminosity conversion to a SFR was from Equations (21) and (22) from this paper, depending on the luminosity of that galaxy. The 24 micron SFR relation

was from Equation (6) which is from $\mathrm{Wu}$ et al. (2005). From Figure 12 we have a comparison between these conversions and the model calibrated star formation rate. The 24 micron luminosity 
is not as good at predicting the bolometric IR luminosity (and the integrated star formation) because it does not sample as close to the cold dust mid-IR emission peak as the 70 micron luminosity. The 70 micron flux is much closer to the peak and is likely a better estimate of the IR luminosity and the obscured SFR.

We estimate the IR excess IRX $=\log _{10}\left(\mathrm{~L}_{\text {dust }} / \mathrm{L}_{\mathrm{UV}}\right)$ and plot it against the $F U V-N U V$ color (Figure 13), similar to Figure 6 in the Johnson et al. (2007a) paper, which presents UV and IR data for a sample of star-forming disk galaxies and starburst galaxies. In an earlier comparison of BCGs with star-forming galaxies, Hicks et al. (2010) found that the cool core BCGs in their sample tended to be bluer in UV color and have a large scatter in IRX compared to those properties in the galaxies in Johnson et al. (2007a). We do see the larger scatter in IRX for those BCGs that have a bluer UV color. We note that most of the BCGs in our plot are found in low central entropy clusters because those are the only BCGs with FUV, NUV, and Spitzer mid-IR detections.

\subsection{Star Formation and Cluster Entropy Profiles}

We have shown here and in previous works (e.g., Cavagnolo et al. 2008, 2009; Rafferty et al. 2008), that BCGs in clusters with low central entropy $\left(K_{0}\right)$ are the only BCGs to exhibit signs of vigorous star formation. The upper threshold for activity in BCGs appears to be around $30 \mathrm{keV}$

$\mathrm{cm}^{2}$. Table 1 presents the subsamples with excess emission. We investigate here to see whether the strength of the signatures of activity, the UV and mid-IR excess, exhibited any trend with the central entropy floor or other cluster property.

Here we take the derived star formation rates as simply indicative of the level of star formation activity. By assuming the star formation is constant, we have taken a nominal assumption about the conversion factors and the starburst models, and translated luminosities into SFRs. We are not claiming that the star formation is continuous. Distinctions between continuous star formations, simple single-burst models of a single age, and convolutions of more complicated star formation histories are well beyond the scope of broad-band photometric data and global measurements. For example, extremely recent star formation is best tracked with $\mathrm{H} \alpha$, but the $\mathrm{H} \alpha$ fluxes available from the literature are typically from long-slit spectra, and therefore can underestimate emission line flux if some of it is located outside the central $2^{\prime \prime}$ or so. $\mathrm{H} \alpha$ can also be affected by dust extinction in heavily obscured regions; $\mathrm{H} \alpha$ can be produced by mechanisms other than by recombination in star formation regions. Mid-IR emission provides a pretty reliable assessment of the obscured star formation energy output, since it is like a bolometric measure of luminosity emitted by dust. At low star formation rates, the colder dust, heated by evolved stars can contribute to the longer wavelength emission, so the lowest IR SFRs in our sample (below about 0.1 solar masses per year) may be regarded as upper limits. The UV light from a galaxy is very sensitive to the presence of hot stars if some of their light escapes the galaxy. We do not attempt to correct the UV light for internal extinction, so the UV and the mid-IR are sampling complementary components of any star formation-related light. 
A sum of the UV and IR SFRs is therefore a best estimate of something akin to the total star formation rate of the $\mathrm{BCG}$, and even the most conservative interpretation is that they indicate the current luminosity of star formation in the BCG. We do not see any correlation between the entropy profile and the strength of star formation signatures (e.g. the UV or the mid-IR luminosities of the BCGs with various X-ray gas quantities, $K_{0}$ or the value of the entropy profile at $20 \mathrm{kpc}(K(r=20 \mathrm{kpc})))$. In Figure 14 we plot the quantities of SFR and $K_{0}$. Upon first glance, there may seem to be a trend for the detected lowest entropy systems to have the lowest star-formation luminosities. However, these are lowest redshift groups in the ACCEPT sample, with lower luminosities and masses overall. They are quite nearby, so the ones that are wellobserved by Chandra have entropy profiles that probe the sub kpc-scales. Excluding the groups (or including the upper limits for BCGs in groups without evidence for star formation activity) erases any semblance of a trend. To test that we were not missing a trend because the best fit $K_{0}$ could be biased high for the more distant clusters (see Cavagnolo et al 2009), we plot $K_{0}$ and SFR for the BCGs with z between 0.05 and 0.15. In this subsample, no trend is visible. Furthermore, the expected trend would be that the lowest entropy systems would have the largest star formation luminosities because the gas has the shortest detected cooling times. Therefore we see no evidence for a simple relation between central gas entropy or cooling time and the estimated SFR.

\subsection{ICM Gas Cooling and Star Formation in BCGs}

While the presence of high density, high pressure intracluster gas seems to be a prerequisite for a BCG to host some star formation, role of the intracluster gas is not quite clear. The current paradigm suggests that some of the hot gas cools and forms stars, but a gas that has been at X-ray temperatures for some time has likely sputtered away any grains it may have had. The lifetime of a typical Galactic dust grain in $10^{7} \mathrm{~K}$ gas is of order 10 million years (Draine \& Salpeter 1979). Dust-free gas forms molecular hydrogen only very slowly (e.g., Bromm et al. 2009). Voit \& Donahue (2011) show that for BCGs with measured reservoirs of $\mathrm{CO}$ (and $\mathrm{H}_{2}$ ), the gas residence time (= $M\left(H_{2}\right) / S F R$ ) for BCGs is very similar to that of star-forming disk galaxies at $\mathrm{SFR}<10 \mathrm{M}_{\odot} \mathrm{yr}^{-1}$, around a Gyr. For BCGs with rapid SFRs, the residence time is similar to that of starbursts with similar SFRs $\left(\sim 10^{7}-10^{8} \mathrm{yrs}\right)$. They calculate that if much of the stellar winds and ejecta of evolved stars in the BCG are retained by the BCG, perhaps as a consequence of the higher intracluster pressures, this gas could fuel much of the existing star formation occuring at a steady rate. Certainly for BCGs with $\mathrm{SFR} \sim 10$ solar masses per year or less, the stellar ejecta is a source of material that has mass of similar order of magnitude to any source of cooled ICM gas.

However, for galaxies with gas reservoirs of $10^{10}$ solar masses or more, cooled ICM appears to be required to supply the molecular clouds. The stellar ejecta or contributions from the ISM of dusty galaxies (e.g. Sparks et al. 1989) may provide dusty seeds that may mix with the ICM and significantly accelerate its cooling. The larger SFRs cannot be sustained at a steady rate, given the gas supply, and just as in starburst galaxies, must be a short-term situation. The gas may 
accumulate over a longer period. Given that $\sim 1 / 3$ of low redshift cool core galaxies exhibit $\mathrm{H} \alpha$, a similar fraction of cool core BCGs (or possibly fewer, if some of the $\mathrm{H} \alpha$ emission is not related to SF) are in the star-forming state. Therefore, such galaxies could accumulate the ejecta of their stellar inhabitants into molecular clouds for Gigayears, then experience a burst once a threshold surface density of molecular hydrogen was achieved.

The empirical correlation between the presence low-entropy ICM and the star formation in the central BCG is incontrovertible. However, the common interpretation of this correlation that cooled ICM fuels the star formation has not been backed up by a physically plausible theory for how the hot ICM cools and makes cold and dusty molecular clouds. The resident stellar population is an obvious source of dust (and cool gas) that should not be neglected.

\section{Conclusions}

We present photometry for brightest cluster galaxies in the ACCEPT cluster sample, derived from GALEX, Spitzer, and 2MASS archival observations. This sample includes 239 clusters which were well-observed by Chandra up until late 2008, with hot gas entropy profiles uniformly extracted (Cavagnolo et al. 2009). We identified the BCGs in all of the clusters. In our BCG identification, it is twice as likely to be within 10 projected kpc of its X-ray centroid in low entropy clusters $(74 \%)$ compared to high entropy clusters $(37 \%)$.

Similar to what has been seen in other star formation indicators (e.g. $\mathrm{H} \alpha$ ), galaxy clusters with low central gas entropies (also known as "cool core" clusters) are the only clusters to host BCGs with infrared and UV excesses above those from the old stellar population. The entropy threshold of $30 \mathrm{keV} \mathrm{cm}^{2}$ is consistent with the entropy threshold identified by other work (Cavagnolo et al. 2008; Rafferty et al. 2008; Cavagnolo et al. 2009). We found 168 observations by the near UV imaging by GALEX, of which 112 BCGs were detected. We found a mean NUV-K (6.59 \pm 0.34$)$ color seen in quiescent BCGs and use that to quantify excess UV emission in individual BCGs. Of the 84 clusters with low central gas entropy , 32 (38\%) hosted BCGs with a UV excess, while none of the clusters with high central gas entropy did. The scatter (i.e. standard deviation) in the NUV-K AB color of BCGs in low entropy clusters is considerably higher at 0.99 . We did not detect a difference between the mean UV color (FUV-NUV) of BCGs (not including those with possible Ly $\alpha$ contamination), within the error, for low and high entropy clusters.

Similarly, we detected excess infrared emission in some BCGs in low gas entropy clusters over a large range of infrared bands (e.g. 4.5, 8.0, 24, and 70 microns) and no excess in BCGs in high central entropy clusters. The mid-IR emission ratios for BCGs (including quiescent BCGs with mid-IR detections) are consistent with, and span a similar range to, galaxies studied in the Sloan Digital Sky Survey (SDSS) galaxies with a range of star forming properties by Johnson et al. (2007b). For example, 24 of the obeserved 56 BCGs (43\%) in low entropy clusters show excess 24

micron to $\mathrm{K}$ band emission. The standard deviation of the ratio $\log _{10}\left(\mathrm{~F}_{24 \mu \mathrm{m}} / \mathrm{F}_{\mathrm{K}}\right)$ is 0.81 in these 
BCGs. We also see a strong correlation between excess 4.5 micron and 8.0 micron fluxes that may indicate correlated PAH emission in both of these bands, when the PAH emission is strong.

The excess emission seen in the UV can be used in conjunction with the IR emission to estimate a total star formation rate, accounting for both obscured and unobscured star formation. The UV and IR estimates give complementary information whereas $\mathrm{H} \alpha$ may be affected by contaminating contributions from other sources (e.g. dust extinction, shocks) or limited by technique (e.g. incomplete spatial coverage in long slit spectroscopy, contamination by N II in narrow band imaging). Additionally, the multi-wavelength coverage (as opposed to single band measurements) can help to further constrain possible sources of the excess emission. We see that the near-IR to far-IR emission is consistent with a combination of a starburst model and an old stellar population. Clear signs of these empirical correlations and significant dust emission in some low entropy clusters can help constrain star formation estimates in these BCGs. Aside from the previously noted upper threshold for activity at $K_{0}=30 \mathrm{keV} \mathrm{cm}{ }^{2}$, we do not detect a correlation between the level of luminosities

or excesses with $K_{0}$ (or equivalently, central cooling time.) However, whether the gas fueling this activity comes from cooling of the ICM or other processes, is not so clear. A significant, massive evolved stellar population in these galaxies may produce dusty gas which may be confined by the hot gas and it may provide the seeds of condensation for the gas from the hot, and presumably dust-free, intracluster medium (Voit \& Donahue 2011).

Support for this research was provided by Spitzer contracts JPL RSA 1377112 (MSU RC065166) and JPL 1353923 (MSU RC065195). M. Donahue and A. Hicks were partially supported by a Long Term Space Astrophysics grant NASA NNG05GD82G (MSU RC062757). We would also like to thank Deborah Haarsma and Luke Leisman for their helpful discussion on BCG identification and Mark Voit for his comments on the text. This research has made use of the SIMBAD database, operated at CDS, Strasbourg, France. This research has made use of the NASA/IPAC Extragalac-

tic Database (NED) which is operated by the Jet Propulsion Laboratory, California Institute of Technology, under contract with the National Aeronautics and Space Administration.

Facility: GALEX, Spitzer (IRAC,MIPS), CTIO:2MASS, FLWO:2MASS

\section{REFERENCES}

Arnaud, M., Rothenflug, R., Boulade, O., Vigroux, L., \& Vangioni-Flam, E. 1992, A\&A, 254, 49

Böhringer, H., et al. 2007, A\&A, 469, 363

Bromm, V., Yoshida, N., Hernquist, L., \& McKee, C. F. 2009, Nature, 459, 49

Burns, J. O. 1990, AJ, 99, 14

Calzetti, D., et al. 2010, ApJ, 714, 1256 
Catinella, B., et al. 2010, MNRAS, 403, 683

Cavagnolo, K. W., Donahue, M., Voit, G. M., \& Sun, M. 2008, ApJ, 683, L107

—. 2009, ApJS, 182, 12

Cohen, M., Wheaton, W. A., \& Megeath, S. T. 2003, AJ, 126, 1090

Cowie, L. L., \& Binney, J. 1977, ApJ, 215, 723

Crawford, C. S., Allen, S. W., Ebeling, H., Edge, A. C., \& Fabian, A. C. 1999, MNRAS, 306, 857

David, L. P., Arnaud, K. A., Forman, W., \& Jones, C. 1990, ApJ, 356, 32

Donahue, M., de Messières, G. E., O'Connell, R. W., Voit, G. M., Hoffer, A., McNamara, B. R., \& Nulsen, P. E. J. 2011, ApJ, 732, 40

Donahue, M., Horner, D. J., Cavagnolo, K. W., \& Voit, G. M. 2006, ApJ, 643, 730

Donahue, M., Mack, J., Voit, G. M., Sparks, W., Elston, R., \& Maloney, P. R. 2000, ApJ, 545, 670

Donahue, M., et al. 2010, ApJ, 715, 881

Draine, B. T., \& Salpeter, E. E. 1979, ApJ, 231, 77

Edge, A. C. 2001, MNRAS, 328, 762

Edge, A. C., et al. 2010a, A\&A, 518

-. 2010b, A\&A, 518, L47+

Egami, E., Rieke, G. H., Fadda, D., \& Hines, D. C. 2006, ApJ, 652, L21

Elston, R., \& Maloney, P. 1994, in Astrophysics and Space Science Library, Vol. 190, Astronomy with Arrays, The Next Generation, ed. I. S. McLean, 169-+

Fabian, A. C. 1994, ARA\&A, 32, 277

Fabian, A. C., \& Nulsen, P. E. J. 1977, MNRAS, 180, 479

Fazio, G. G., et al. 2004, ApJS, 154, 10

Ferrari, C., Arnaud, M., Ettori, S., Maurogordato, S., \& Rho, J. 2006, A\&A, 446, 417

Gonzalez, A. H., Zaritsky, D., \& Zabludoff, A. I. 2007, ApJ, 666, 147

Groves, B., Dopita, M. A., Sutherland, R. S., Kewley, L. J., Fischera, J., Leitherer, C., Brandl, B., \& van Breugel, W. 2008, ApJS, 176, 438

Heckman, T. M., Baum, S. A., van Breugel, W. J. M., \& McCarthy, P. 1989, ApJ, 338, 48 
Hicks, A. K., Mushotzky, R., \& Donahue, M. 2010, ApJ, 719, 1844

Hu, E. M., Cowie, L. L., \& Wang, Z. 1985, ApJS, 59, 447

Jaffe, W., \& Bremer, M. N. 1997, MNRAS, 284, L1

Jarrett, T. H., Chester, T., Cutri, R., Schneider, S. E., \& Huchra, J. P. 2003, AJ, 125, 525

Johnson, B. D., et al. 2007a, ApJS, 173, 377

-. 2007b, ApJS, 173, 392

Kennicutt, Jr., R. C. 1998, ARA\&A, 36, 189

Kennicutt, Jr., R. C., et al. 2003, PASP, 115, 928

Leisman, L., Haarsma, D. B., Sebald, D. A., \& ACCEPT team. 2011, in Bulletin of the American Astronomical Society, Vol. 43, American Astronomical Society Meeting Abstracts \#217, \#149.08-+

Makovoz, D., \& Marleau, F. R. 2005, PASP, 117, 1113

Martin, D. C., et al. 2005, ApJ, 619, L1

Morrissey, P., et al. 2007, ApJS, 173, 682

O'Connell, R. W. 1999, ARA\&A, 37, 603

O'Dea, C. P., et al. 2008, ApJ, 681, 1035

O’Dea, K. P., et al. 2010, ApJ, 719, 1619

Peterson, J. R., Kahn, S. M., Paerels, F. B. S., Kaastra, J. S., Tamura, T., Bleeker, J. A. M., Ferrigno, C., \& Jernigan, J. G. 2003, ApJ, 590, 207

Quillen, A. C., et al. 2008, ApJS, 176, 39

Rafferty, D. A., McNamara, B. R., \& Nulsen, P. E. J. 2008, ApJ, 687, 899

Reach, W. T., et al. 2005, PASP, 117, 978

Reiprich, T. H., \& Böhringer, H. 2002, ApJ, 567, 716

Rieke, G. H., et al. 2004, ApJS, 154, 25

Sanderson, A. J. R., Edge, A. C., \& Smith, G. P. 2009, MNRAS, 398, 1698

Schlegel, D. J., Finkbeiner, D. P., \& Davis, M. 1998, ApJ, 500, 525

Sparks, W. B., Paresce, F., \& Macchetto, D. 1989, ApJ, 347, L65 
Sun, M. 2009, ApJ, 704, 1586

Sun, M., Jones, C., Forman, W., Vikhlinin, A., Donahue, M., \& Voit, M. 2007, ApJ, 657, 197

Voit, G. M., Cavagnolo, K. W., Donahue, M., Rafferty, D. A., McNamara, B. R., \& Nulsen, P. E. J. 2008, ApJ, 681, L5

Voit, G. M., \& Donahue, M. 2011, ApJ, 738, L24+

Wang, J., Overzier, R., Kauffmann, G., von der Linden, A., \& Kong, X. 2010, MNRAS, 401, 433

Wu, H., Cao, C., Hao, C.-N., Liu, F.-S., Wang, J.-L., Xia, X.-Y., Deng, Z.-G., \& Young, C. K.-S. 2005, ApJ, 632, L79 
Table 1. Summary of Observations and Detections

\begin{tabular}{ccccc}
\hline \hline Waveband & Observations & Observations $\left(K_{0} \leq 30 \mathrm{keV} \mathrm{cm}^{2}\right)$ & Detections & Excess \\
\hline NUV & 168 & 84 & 112 & $32^{\mathrm{a}}$ \\
4.5 micron & 76 & 52 & 76 & 13 \\
8.0 micron & 76 & 52 & 76 & 43 \\
24 micron & 98 & 56 & 94 & $24^{\mathrm{b}}$ \\
70 micron & 65 & 46 & 32 & 32 \\
160 micron & 33 & 21 & 16 & 16 \\
\hline
\end{tabular}

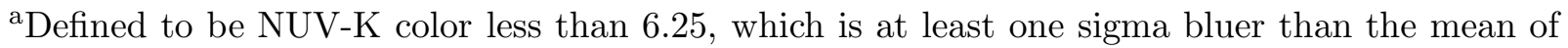
the quiescent BCGs.

${ }^{\mathrm{b}}$ Defined to be a 24 micron to $\mathrm{K}$ band ratio greater than 0.113 , which is at least one sigma greater than the mean of the quiescent BCGs.

Note. - Refer to appropriate section of text for definitions of Detections and Excess. 
Table 2. Brightest Cluster Galaxy Identifications.

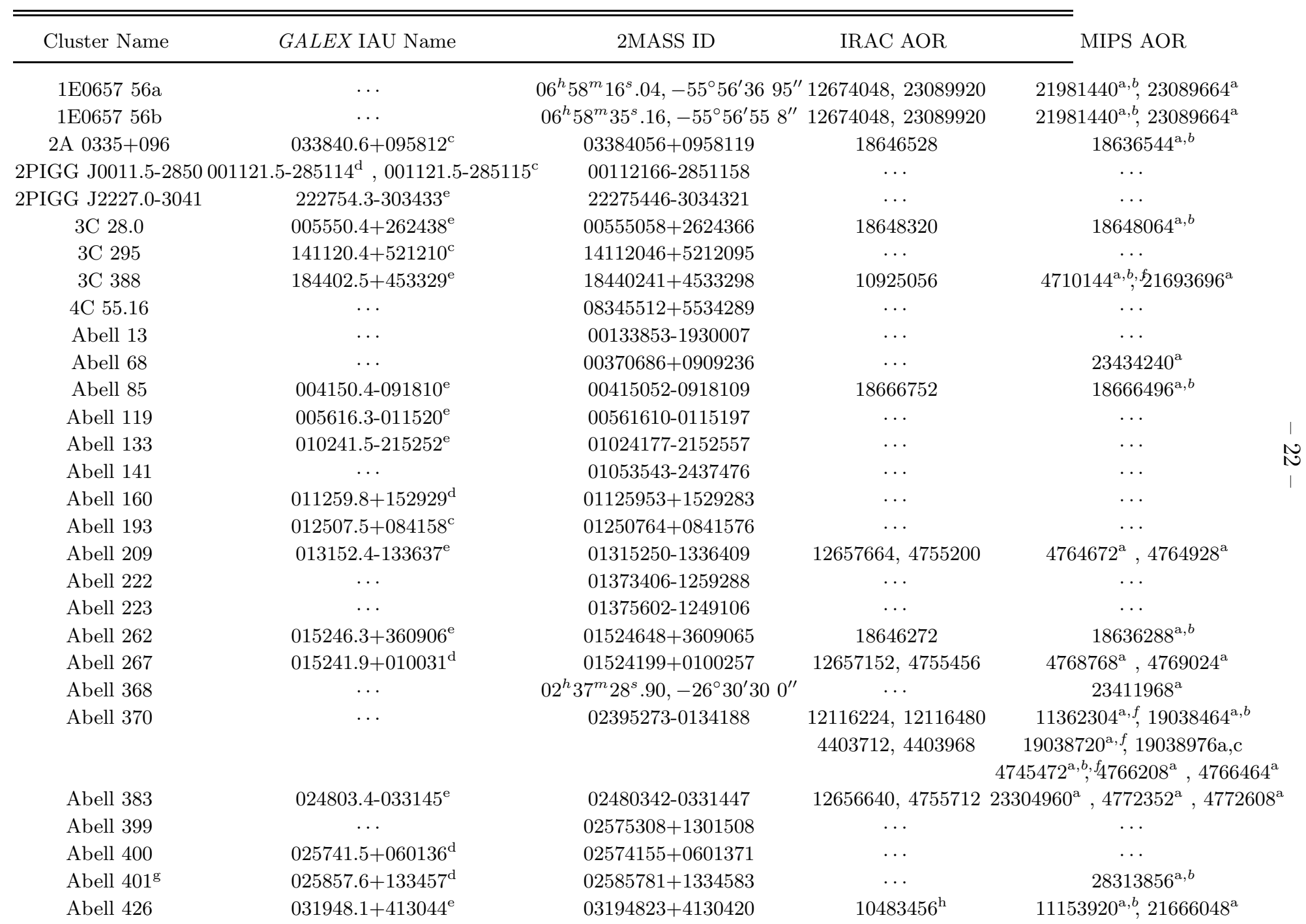


Table 2-Continued

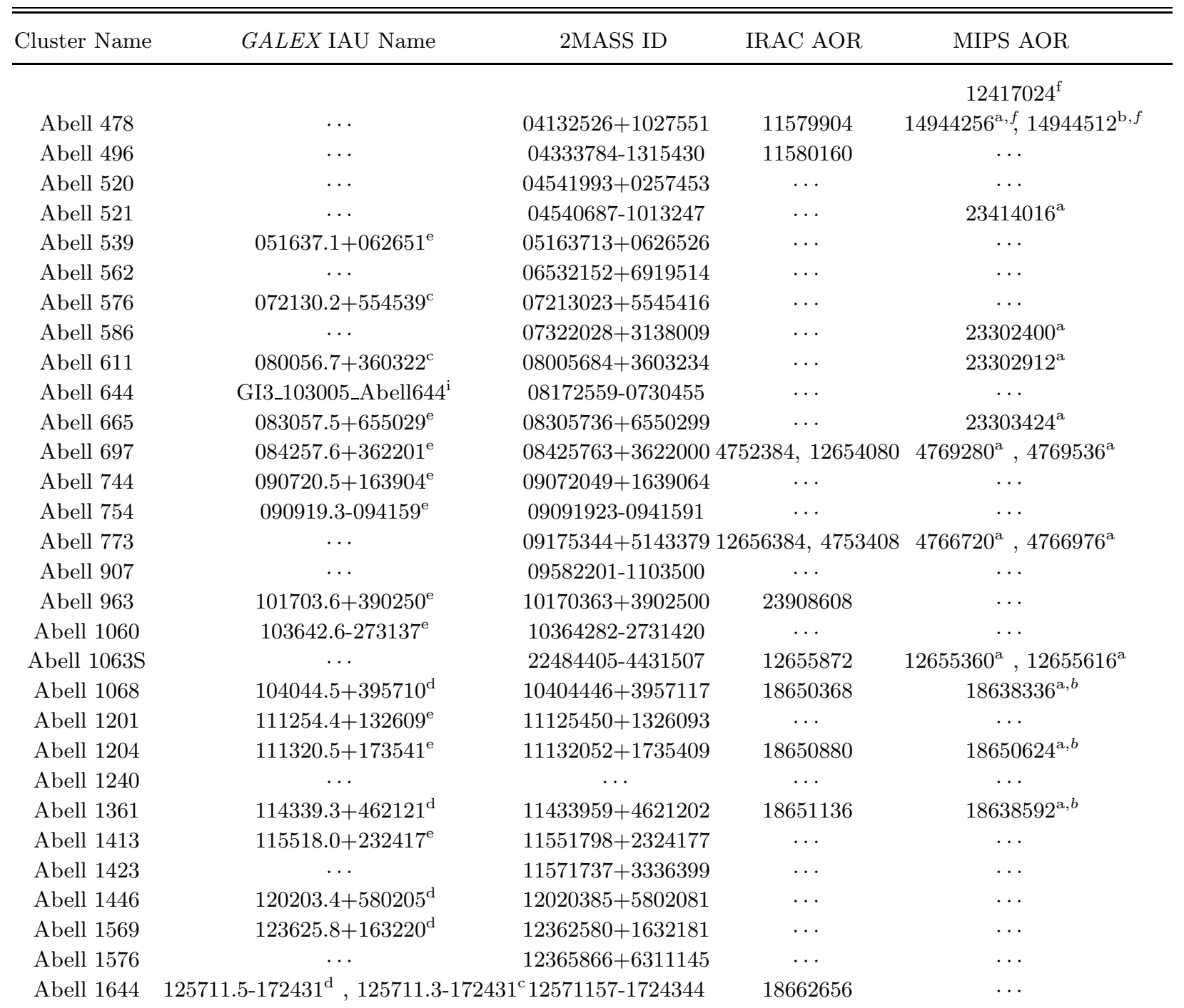


Table 2-Continued

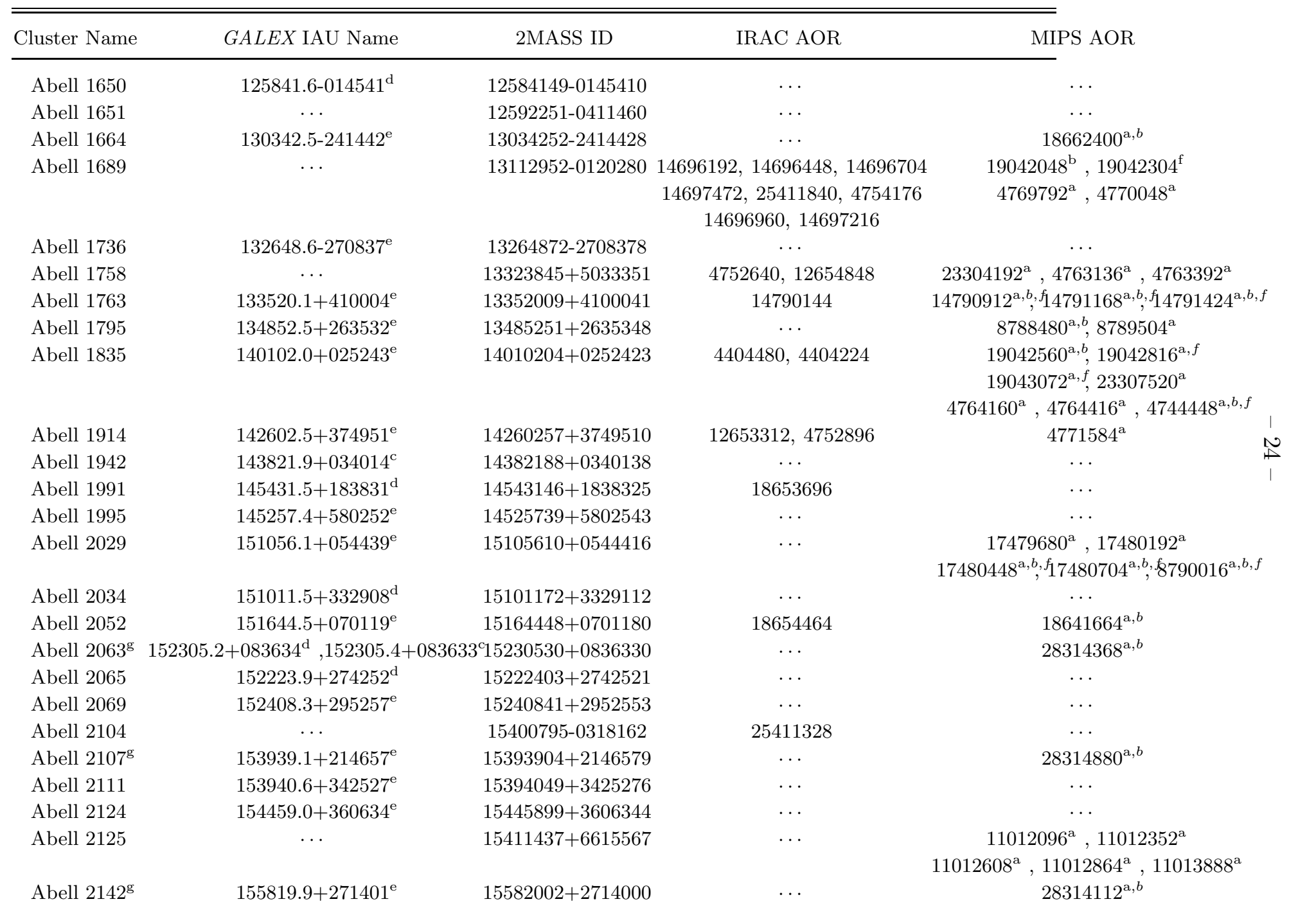


Table 2-Continued

\begin{tabular}{|c|c|c|c|c|}
\hline Cluster Name & $G A L E X$ IAU Name & 2MASS ID & IRAC AOR & MIPS AOR \\
\hline Abell 2147 & $160217.1+155831^{\mathrm{e}}$ & $16021700+1558282$ & $\ldots$ & $\ldots$ \\
\hline Abell 2151 & $160435.7+174318^{\mathrm{e}}$ & $16043575+1743172$ & $\ldots$ & $3860480^{\mathrm{a}, b, f}$ \\
\hline Abell 2163 & $\ldots$ & $16^{h} 15^{m} 33^{s} .60,-06^{\circ} 09^{\prime} 16^{\prime \prime}$ & $\cdots$ & $\ldots$ \\
\hline Abell 2187 & $162413.7+411433^{\mathrm{e}}$ & $16241402+4114377$ & $\ldots$ & $\ldots$ \\
\hline Abell 2199 & $162838.3+393304^{\mathrm{d}}, 162838.2+393304^{\mathrm{c}}$ & $16283827+3933049$ & $3860992^{\mathrm{h}}, 3861248^{\mathrm{h}}$ & $14957056^{\mathrm{a}, f}$ \\
\hline Abell 2204 & $\ldots$ & $16324698+0534410$ & 18655232 & $18642432^{\mathrm{a}, b}$ \\
\hline Abell 2218 & $163549.3+661244$ & $16^{h} 35^{m} 49^{s} .39,66^{\circ} 12^{\prime} 451^{\prime \prime}$ & $\begin{array}{c}14697728,14698240 \\
14698496,4751616\end{array}$ & $\begin{array}{c}19044096^{\mathrm{f}}, 19044352^{\mathrm{b}}, 19044608^{\mathrm{f}} \\
4763648^{\mathrm{a}}, 4763904^{\mathrm{a}}\end{array}$ \\
\hline Abell 2219 & $164019.8+464242^{\mathrm{e}}$ & $16401981+4642409$ & 4756224 & $23308544^{\mathrm{a}}, 4773376^{\mathrm{a}}, 4773632^{\mathrm{a}}$ \\
\hline Abell 2244 & $\ldots$ & $17024247+3403363$ & $\ldots$ & $\ldots$ \\
\hline Abell 2255 & $171229.1+640338^{\mathrm{e}}$ & $17122875+6403385$ & $\ldots$ & $21778944^{\mathrm{a}, b}$ \\
\hline Abell 2256 & $170427.0+783826^{\mathrm{e}}$ & $17042724+7838260$ & $\cdots$ & $\ldots$ \\
\hline Abell 2259 & $\ldots$ & $17200968+2740077$ & $\ldots$ & $\cdots$ \\
\hline Abell 2261 & $\ldots$ & $17222717+3207571$ & 12655104,4752128 & $4744960^{\mathrm{b}, f}, 47651,84^{\mathrm{a}}, 4765440^{\mathrm{a}}$ \\
\hline Abell 2294 & $\ldots$ & $17241223+8553116$ & $\ldots$ & $\ldots$ F \\
\hline Abell 2319 & $192109.9+435647^{\mathrm{c}}$ & $19211004+4356443$ & $\ldots$ & $\ldots$ \\
\hline Abell 2384 & $\ldots$ & 21522195-1932484 & $\ldots$ & $\ldots$ \\
\hline Abell 2390 & $215336.8+174144^{\mathrm{e}}$ & $21533687+1741439$ & $\begin{array}{c}12658688,23473664^{\mathrm{j}}, 4754432 \\
23475712^{\mathrm{k}}, 23475968^{\mathrm{k}}\end{array}$ & $\begin{array}{c}19044864^{\mathrm{b}}, 19045120^{\mathrm{f}} \\
4743680^{\mathrm{b}}, 4762624^{\mathrm{a}}, 4762880^{\mathrm{a}}\end{array}$ \\
\hline Abell 2409 & $\ldots$ & $22005255+2058087$ & $\ldots$ & $\ldots$ \\
\hline Abell 2420 & $\ldots$ & $22101878-1210141$ & $\cdots$ & $\cdots$ \\
\hline Abell 2462 & $223911.4-172029^{\mathrm{e}}$ & $22391136-1720284$ & $\cdots$ & $\ldots$ \\
\hline Abell 2537 & $\ldots$ & $23082221-0211315$ & $\ldots$ & $23435008^{\mathrm{a}}$ \\
\hline Abell 2554 & $231219.9-213007^{\mathrm{d}}$ & 23121995-2130098 & $\ldots$ & $\ldots$ \\
\hline Abell 2556 & $231301.3-213804^{\mathrm{e}}$ & 23130142-2138039 & $\ldots$ & $\ldots$ \\
\hline Abell 2589 & $232357.2+164637^{\mathrm{d}}, 232357.4+1646382$ & $23235741+1646379$ & $\ldots$ & $\ldots$ \\
\hline Abell 2597 & $232519.7-120726^{\mathrm{e}}$ & $23251973-1207275$ & 13372160 & $13371904^{\mathrm{a}, b, f}, 17791744^{\mathrm{a}, b}, 17792000^{\mathrm{a}, b}$ \\
\hline Abell 2626 & $233630.6+210848^{\mathrm{e}}$ & $23363057+2108498$ & 18657280 & $18644480^{\mathrm{a}, b}$ \\
\hline Abell 2631 & $233739.7+001616^{\mathrm{d}}$ & $23373975+0016165$ & $\ldots$ & $\ldots$ \\
\hline Abell 2657 & $234457.4+091134^{\mathrm{d}}$ & $23445742+0911349$ & $\ldots$ & $\ldots$ \\
\hline Abell 2667 & $235139.4-260501^{\mathrm{e}}$ & $23513947-2605032$ & $23476224,12656896,23474688,4756992$ & $4775424^{\mathrm{a}}, 4775680^{\mathrm{a}}$ \\
\hline
\end{tabular}


Table 2-Continued

\begin{tabular}{|c|c|c|c|c|}
\hline Cluster Name & $G A L E X$ IAU Name & 2MASS ID & IRAC AOR & MIPS AOR \\
\hline Abell 2717 & $000313.0-355613^{\mathrm{e}}$ & 00031298-3556132 & $\cdots$ & \\
\hline Abell 2744a & $\ldots$ & $00^{h} 14^{m} 20^{s} .677,-30^{\circ} 24^{\prime} 0015^{\prime \prime}$ & 12653568,4753920 & $11359232^{\mathrm{b}, f}, 4765696^{\mathrm{a}}, 4765952^{\mathrm{a}}$ \\
\hline Abell 2744b & $\cdots$ & $00^{h} 14^{m} 22^{s} .0,-30^{\circ} 24^{\prime} 20^{\prime \prime}$ & 12653568,4753920 & $11359232^{\mathrm{b}, f}, 4765696^{\mathrm{a}}, 4765952^{\mathrm{a}}$ \\
\hline Abell 2813 & $\ldots$ & 00432504-2037054 & $\ldots$ & $\cdots$ \\
\hline Abell 3084 & $030403.7-365629^{\mathrm{e}}$ & 03040396-3656274 & $\cdots$ & $23412224^{\mathrm{a}}$ \\
\hline Abell 3088 & $\ldots$ & 03070207-2839574 & $\ldots$ & $23412480^{\mathrm{a}}$ \\
\hline Abell 3112 & $031757.6-441417^{\mathrm{e}}$ & 03175766-4414175 & 18667264 & $18667008^{\mathrm{a}, b}$ \\
\hline Abell 3120 & $032156.0-511935^{\mathrm{e}}$ & 03215645-5119357 & $\ldots$ & $\ldots$ \\
\hline Abell 3158 & $034252.9-533752^{\mathrm{e}}$ & 03425295-5337526 & $\ldots$ & $8788736^{\mathrm{a}, b, f}$ \\
\hline Abell 3266 & $043113.3-612713^{\mathrm{e}}$ & 04311330-6127114 & $\ldots$ & $8788992^{\mathrm{a}, b,}, 8789760^{\mathrm{a}}$ \\
\hline Abell 3364 & $\ldots$ & 05473773-3152237 & $\ldots$ & $23416832^{\mathrm{a}}$ \\
\hline Abell 3376 & $060209.3-395702^{\mathrm{e}}$ & 06020973-3956597 & $\ldots$ & $\ldots$ \\
\hline Abell 3391 & $\ldots$ & $06262045-5341358$ & $\ldots$ & $14426880^{\mathrm{a}, b, f}$ \\
\hline Abell 3395 & $\ldots$ & 06264958-5432340 & $\ldots$ & $\ldots$ \\
\hline Abell 3528S & $125441.1-291340^{\mathrm{e}}$ & $12544100-2913397$ & $\ldots$ & $\ldots$ \\
\hline Abell 3558 & $132756.8-312946^{\mathrm{e}}$ & $13275688-3129437$ & $\ldots$ & $\ldots$ \\
\hline Abell 3562 & $133334.8-314019^{\mathrm{d}}$ & 13333473-3140201 & $\cdots$ & $\cdots$ \\
\hline Abell 3571 & $\ldots$ & $13472838-3251540$ & $\ldots$ & $\ldots$ \\
\hline Abell 3581 & $140729.7-270104^{\mathrm{e}}$ & $14072978-2701043$ & $\ldots$ & $\ldots$ \\
\hline Abell 3667 & $\ldots$ & $20122726-5649363$ & $\cdots$ & $\cdots$ \\
\hline Abell 3822 & $215404.1-575203^{\mathrm{e}}$ & $21540421-5752033$ & $\ldots$ & $\ldots$ \\
\hline Abell 3827 & $220153.0-595645^{\mathrm{e}}$ & $22015330-5956437$ & $\ldots$ & $\ldots$ \\
\hline Abell 3921 & $224957.9-642547^{\mathrm{e}}$ & $22495818-6425468$ & $\ldots$ & $\ldots$ \\
\hline Abell 4038 & $\ldots$ & 23474504-2808265 & $\ldots$ & $\ldots$ \\
\hline Abell 4059 & $235700.7-344531^{\mathrm{d}}$ & $23570068-3445331$ & 18666240 & $18665984^{\mathrm{a}, b}$ \\
\hline Abell S0405 & $035133.9-821312^{\mathrm{e}}$ & 03513399-8213103 & $\ldots$ & $\ldots$ \\
\hline Abell S0592 & $\ldots$ & $06384867-5358246$ & $\ldots$ & $23417600^{\mathrm{a}}$ \\
\hline AC 114 & $\ldots$ & $22584841-3448087$ & $4756480,25412864,12653824$ & $4773888^{\mathrm{a}}, 4774144^{\mathrm{a}}$ \\
\hline AWM7 & $025427.5+413448^{\mathrm{d}}$ & $02542739+4134467$ & $\cdots$ & $\ldots$ \\
\hline CENTAURUS & $124849.4-411838^{\mathrm{e}}$ & $12484927-4118399$ & 11042816 & $11043328^{\mathrm{a}, b, f}$ \\
\hline CID 0072 & $173301.8+434535^{\mathrm{e}}$ & $17330202+4345345$ & 18656000 & $18643200^{\mathrm{a}, b}$ \\
\hline
\end{tabular}


Table 2-Continued

\begin{tabular}{|c|c|c|c|c|}
\hline Cluster Name & $G A L E X$ IAU Name & 2MASS ID & IRAC AOR & MIPS AOR \\
\hline CL J1226.9+3332 & $122658.7+333249^{\mathrm{d}}$ & $12^{h} 26^{m} 58^{s} .24,33^{\circ} 32^{\prime} 486^{\prime \prime}$ & $\begin{array}{c}15062784^{\mathrm{k}}, 15063040^{\mathrm{j}} \\
22555904^{\mathrm{k}}, 22556160^{\mathrm{k}}, 3815680\end{array}$ & $\begin{array}{c}4761088^{\mathrm{a}}, 4761344 \\
4748032^{\mathrm{b}, f}\end{array}$ \\
\hline CYGNUS A & $\cdots$ & $19592833+4044022$ & $14265344^{\mathrm{k}}$ & $21694208^{\mathrm{a}}, 4710656^{\mathrm{a}, b, f}$ \\
\hline ESO 3060170 & $054006.4-405009^{\mathrm{d}}$ & 05400667-4050114 & $\ldots$ & $\ldots$ \\
\hline ESO 5520200 & $045452.5-180657^{\mathrm{e}}$ & 04545226-1806561 & $\ldots$ & $\cdots$ \\
\hline EXO 0422-086 & $042551.8-083330^{\mathrm{e}}$ & 04255133-0833389 & $\cdots$ & $\ldots$ \\
\hline HCG 0062 & $125305.7-091213^{\mathrm{c}}$ & $12530567-0912141$ & 11251712 & $11248640^{\mathrm{a}, b}$ \\
\hline HCG 42 & $100014.1-193809^{\mathrm{e}}$ & $10001412-1938113$ & $11250688^{\mathrm{j}}, 11253248^{\mathrm{k}}$ & $11247616^{\mathrm{a}, b}$ \\
\hline HERCULES A & $165108.1+045933^{\mathrm{e}}$ & $16510802+0459349$ & $26922496^{\mathrm{k}}$ & $21692928^{\mathrm{a}}$ \\
\hline HYDRA A & $091805.5-120544^{\mathrm{d}}, 091805.6-120543^{\mathrm{c}}$ & 09180565-1205439 & $26923008^{\mathrm{k}}$ & $21691136^{\mathrm{a}}, 4707584^{\mathrm{a}, b, f}$ \\
\hline M49 & $122946.6+080000^{\mathrm{d}}, 122946.7+080006^{\mathrm{c}}$ & $12294679+0800014$ & $4469760^{\mathrm{h}}$ & $4470272^{\mathrm{a}, b, f}$ \\
\hline M87 & $123049.4+122328^{\mathrm{e}}$ & $12304942+1223279$ & $12673792^{\mathrm{h}}$ & $11375872^{\mathrm{a}}, 21683200^{\mathrm{a}}, 4693248^{\mathrm{a}, b, f}$ \\
\hline MACS J0011.7-1523 & $\ldots$ & $\ldots$ & $\ldots$ & $\cdots$ \\
\hline MACS J0035.4-2015 & $\ldots$ & $00352611-2015440$ & $\ldots$ & 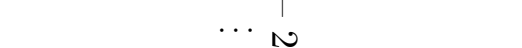 \\
\hline MACS J0159.8-0849 & $\ldots$ & $\ldots$ & $\ldots$ & $\ldots \stackrel{-v}{-}$ \\
\hline MACS J0242.5-2132 & $\cdots$ & $\cdots$ & $\cdots$ & $\ldots$ \\
\hline MACS J0257.1-2325 & $\cdots$ & $\cdots$ & $\cdots$ & $\cdots$ \\
\hline MACS J0257.6-2209 & $\cdots$ & 02574108-2209179 & $\cdots$ & $\cdots$ \\
\hline MACS J0308.9+2645 & $\ldots$ & $03085592+2645373$ & $\ldots$ & $\ldots$ \\
\hline MACS J0329.6-0211 & $\cdots$ & $03^{h} 29^{m} 41^{s} .68,-02^{\circ} 11^{\prime} 489^{\prime \prime}$ & $\cdots$ & $\cdots$ \\
\hline MACS J0417.5-1154 & $\cdots$ & $\cdots$ & $\cdots$ & $\cdots$ \\
\hline MACS J0429.6-0253 & $\cdots$ & 04293604-0253073 & $\cdots$ & $\cdots$ \\
\hline MACS J0520.7-1328 & $\cdots$ & 05204205-1328477 & $\cdots$ & $\cdots$ \\
\hline MACS J0547.0-3904 & $\cdots$ & $\ldots$ & $\ldots$ & $\ldots$ \\
\hline MACS J0717.5+3745 & $\cdots$ & $\cdots$ & 23163648 & $23164160,23163904,23164928$ \\
\hline MACS J0744.8+3927 & $\ldots$ & $07^{h} 44^{m} 51^{s} .98,39^{\circ} 27^{\prime} 351^{\prime \prime}$ & $\ldots$ & $\ldots$ \\
\hline MACS J1115.2+5320 & $\ldots$ & $\ldots$ & $\cdots$ & $\ldots$ \\
\hline MACS J1115.8+0129 & $\cdots$ & $\ldots$ & $\cdots$ & $\cdots$ \\
\hline MACS J1131.8-1955 & $\cdots$ & 11315413-1955391 & $\cdots$ & $\cdots$ \\
\hline MACS J1149.5+2223 & $\cdots$ & $11^{h} 49^{m} 36^{s}, 22^{\circ} 23^{\prime} 53^{\prime \prime}$ & $\cdots$ & $\cdots$ \\
\hline MACS J1206.2-0847 & $\cdots$ & $\ldots$ & $\cdots$ & $\ldots$ \\
\hline
\end{tabular}


Table 2-Continued

\begin{tabular}{|c|c|c|c|c|}
\hline Cluster Name & GALEX IAU Name & 2MASS ID & IRAC AOR & MIPS AOR \\
\hline MACS J1311.0-0310 & $\cdots$ & $\ldots$ & $\cdots$ & $\ldots$ \\
\hline MACS J1621.3+3810 & $\ldots$ & $\ldots$ & $\ldots$ & $\ldots$ \\
\hline MACS J1931.8-2634 & $\ldots$ & $\ldots$ & $\cdots$ & $\cdots$ \\
\hline MACS J2049.9-3217 & $\ldots$ & $\ldots$ & $\ldots$ & $\ldots$ \\
\hline MACS J2211.7-0349 & $\ldots$ & 22114596-0349438 & $\ldots$ & $23433984^{\mathrm{a}}$ \\
\hline MACS J2214.9-1359 & $\ldots$ & $22^{h} 14^{m} 56^{s} .51,14^{\circ} 00^{\prime} 172^{\prime \prime}$ & $\ldots$ & $\ldots$ \\
\hline MACS J2228+2036 & $\ldots$ & $\ldots$ & $\ldots$ & $\ldots$ \\
\hline MACS J2229.7-2755 & $222945.2-275536^{\mathrm{e}}$ & $22294524-2755353$ & $\cdots$ & $\cdots$ \\
\hline MACS J2245.0+2637 & $\ldots$ & $22450463+2638039$ & $\ldots$ & $\ldots$ \\
\hline MKW3S & $152151.8+074229^{\mathrm{e}}$ & $15215187+0742319$ & $\ldots$ & $\ldots$ \\
\hline MKW 04 & $120427.0+015344^{\mathrm{e}}$ & $12042705+0153456$ & $18034432^{\mathrm{h}}$ & $18042624^{\mathrm{a}, b, f}$ \\
\hline MKW 08 & $144042.8+032755^{\mathrm{e}}$ & $14404287+0327555$ & $\ldots$ & $\ldots$ \\
\hline MS 0016.9+1609 & $\ldots$ & $00^{h} 18^{m} 33^{s} .6,16^{\circ} 26^{\prime} 15^{\prime \prime}$ & 4756736,12656128 & $\ldots$ \\
\hline MS 0116.3-0115 & $011853.6-010006^{\mathrm{e}}$ & 01185363-0100074 & $\ldots$ & $\ldots$ \\
\hline MS 0440.5+0204 & $044310.1+021016^{\mathrm{d}}$ & $04430994+0210190$ & $\ldots$ & $\ldots$ \\
\hline MS 0451.6-0305 & $\ldots$ & $04^{h} 54^{m} 10^{s} .85,-03^{\circ} 00^{\prime} 519^{\prime \prime}$ & 12652800,4753664 & $\begin{array}{c}19039232^{\mathrm{b}}, 19039488^{\mathrm{f}} \\
19039744^{\mathrm{b}}, 19040000^{\mathrm{f}} \\
4747520^{\mathrm{b}, f}, 4770304^{\mathrm{a}}, 4770560^{\mathrm{a}}\end{array}$ \\
\hline MS 0735.6+7421 & $074144.1+741440^{\mathrm{e}}$ & $07414444+7414395$ & 7858688 & $\ldots$ \\
\hline MS 0839.8+2938 & $084256.0+292728^{\mathrm{e}}$ & $08425596+2927272$ & $\ldots$ & $23303936^{\mathrm{a}}, 23418880^{\mathrm{a}}$ \\
\hline MS 0906.5+1110 & $\ldots$ & $09091273+1058286$ & $\ldots$ & $23419136^{\mathrm{a}}$ \\
\hline MS $1006.0+1202$ & $\cdots$ & $10084771+1147379$ & $\ldots$ & $\ldots$ \\
\hline MS 1008.1-1224 & $\ldots$ & $10103233-1239524$ & 25410304 & $\ldots$ \\
\hline MS $1455.0+2232$ & $145715.1+222033^{\mathrm{e}}$ & $14571507+2220341$ & 12657408,4753152 & $4767744^{\mathrm{a}}, 4768000^{\mathrm{a}}$ \\
\hline MS 2137.3-2353 & $214015.1-233939^{\mathrm{e}}$ & $21401517-2339398$ & 12658944,4755968 & $4772864^{\mathrm{a}}, 4773120^{\mathrm{a}}$ \\
\hline MS J1157.3+5531 & $115952.3+553205^{\mathrm{e}}$ & $11595215+5532053$ & $\ldots$ & $\ldots$ \\
\hline NGC 0507 & $012340.1+331522^{\mathrm{e}}$ & $01233995+3315222$ & $18030080^{\mathrm{h}}$ & $14253824^{\mathrm{a}, f}, 14254080^{\mathrm{a}, b}$ \\
\hline NGC 4636 & $124249.8+024117^{\mathrm{e}}$ & $12424986+0241160$ & 4475136 & $4475648^{\mathrm{a}, b, f}$ \\
\hline NGC 5044 & $131523.7-162305^{\mathrm{e}}$ & $13152396-1623079$ & 15573504 & $14258432^{\mathrm{a}, f}, 14258688^{\mathrm{a}, b}$ \\
\hline NGC 5813 & $150111.4+014208^{\mathrm{e}}$ & $15011126+0142070$ & $4490496^{\mathrm{h}}$ & $4491008^{\mathrm{a}, b, f}$ \\
\hline NGC 5846 & $150629.4+013621^{\mathrm{e}}$ & $15062925+0136202$ & $16310272^{\mathrm{h}}, 4491264$ & $4491776^{\mathrm{a}, b, f}$ \\
\hline
\end{tabular}


Table 2-Continued

\begin{tabular}{|c|c|c|c|c|}
\hline Cluster Name & $G A L E X$ IAU Name & 2MASS ID & IRAC AOR & MIPS AOR \\
\hline OPHIUCHUS & $171227.6-232209^{\mathrm{d}}$ & 17122774-2322108 & $\ldots$ & $\ldots$ \\
\hline PKS 0745-191 & $\ldots$ & 07473129-1917403 & 18667776 & $18667520^{\mathrm{a}, b}$ \\
\hline RBS 0461 & $\ldots$ & $03411752+1523477$ & $\ldots$ & $\ldots$ \\
\hline RBS 0533 & $041937.8+022435^{\mathrm{e}}$ & $04193792+0224355$ & $\cdots$ & $\cdots$ \\
\hline RBS 0797 & $094712.4+762313^{\mathrm{e}}$ & $09^{h} 47^{m} 12^{s} \cdot 6,76^{\circ} 23^{\prime} 14^{\prime \prime}$ & $\ldots$ & $\ldots$ \\
\hline RCS J2327-0204 & $232729.2-020323^{\mathrm{e}}$ & $23^{h} 27^{m} 29^{s} .0,-02^{\circ} 03^{\prime} 23^{\prime \prime}$ & $\ldots$ & $\ldots$ \\
\hline RXCJ0331.1-2100 & $\ldots$ & 03310587-2100326 & $\ldots$ & $23412736^{\mathrm{a}}$ \\
\hline RXC J1023.8-2715 & $102350.0-271523^{\mathrm{e}}$ & 10235019-2715232 & $\cdots$ & $\ldots$ \\
\hline RX J0220.9-3829 & $\ldots$ & 02205660-3828475 & $\ldots$ & $\ldots$ \\
\hline RX J0232.2-4420 & $\ldots$ & $\ldots$ & $\ldots$ & $\ldots$ \\
\hline RX J0439+0520 & $043902.2+052047^{\mathrm{d}}$ & $04390223+0520443$ & 18649344 & $18649088^{\mathrm{a}, b}$ \\
\hline RX J0439.0+0715 & $\ldots$ & $04390053+0716038$ & $\ldots$ & $\ldots$ \\
\hline RX J0528.9-3927 & $\ldots$ & $\ldots$ & 33327104 & $23415552^{\mathrm{a}}$ \\
\hline RX J0647.7+7015 & $\ldots$ & $06^{h} 47^{m} 51^{s} .45,70^{\circ} 15^{\prime} 044^{\prime \prime}$ & $\ldots$ & $\ldots$ \\
\hline RX J0819.6+6336 & $081925.9+633726^{\mathrm{d}}$ & $08192591+6337256$ & $\ldots$ & $\cdots$ \\
\hline RX J1000.4+4409 & $100031.2+440844^{\mathrm{d}}$ & $10^{h} 00^{m} 31^{s} \cdot 0,44^{\circ} 08^{\prime} 43^{\prime \prime}$ & $\ldots$ & $\ldots$ \\
\hline RX J1022.1+3830 & $102210.3+383104^{\mathrm{d}}$ & $10221031+3831045$ & $\ldots$ & $\ldots$ \\
\hline RX J1130.0+3637 & $113003.3+363812^{\mathrm{d}}$ & $11300319+3638135$ & $\cdots$ & $\cdots$ \\
\hline RX J1320.2+3308 & $132014.6+330835^{\mathrm{c}}$ & $13201472+3308359$ & $\ldots$ & $\ldots$ \\
\hline RX J1347.5-1145 & $134730.7-114509^{\mathrm{e}}$ & $13^{h} 47^{m} 31^{s} .0,-11^{\circ} 45^{\prime} 09^{\prime \prime}$ & 12654336 & $\cdots$ \\
\hline RX J1423.8+2404 & $\ldots$ & $\ldots$ & $\ldots$ & $\ldots$ \\
\hline RX J1504.1-0248 & $150407.6-024815^{\mathrm{e}}$ & 15040752-0248161 & $\ldots$ & $23426048^{\mathrm{a}}$ \\
\hline RX J1532.9+3021 & $\ldots$ & $15^{h} 32^{m} 53^{s} .8,30^{\circ} 21^{\prime} 00^{\prime \prime}$ & 18658560 & $18658304^{\mathrm{a}, b}$ \\
\hline RX J1539.5-8335 & $\ldots$ & 15393387-8335215 & $\ldots$ & $\ldots$ \\
\hline RX J1720.1+2638 & $172010.0+263730^{\mathrm{e}}$ & $17201001+2637317$ & $\cdots$ & $18659072^{\mathrm{a}, b}$ \\
\hline RX J1720.2+3536 & $\ldots$ & $\ldots$ & $\ldots$ & $\ldots$ \\
\hline RX J1852.1+5711 & $185208.5+571144^{\mathrm{d}}$ & $18520859+5711430$ & $\ldots$ & $\ldots$ \\
\hline RX J2129.6+0005 & $212939.9+000522^{\mathrm{e}}$ & $21293995+0005207$ & 18659584 & $18659328^{\mathrm{a}, b}$ \\
\hline SC 1327-312 & $132947.5-313625^{\mathrm{e}}$ & $13294775-3136250$ & $\ldots$ & $\ldots$ \\
\hline SERSIC 159-03 & $231358.8-424336^{\mathrm{e}}$ & $23135863-4243393$ & 18665728 & $18665472^{\mathrm{a}, \mathrm{b}}$ \\
\hline SS2B153 & $105026.1-125041^{\mathrm{e}}$ & $10502610-1250422$ & $\ldots$ & $\ldots$ \\
\hline
\end{tabular}


Table 2-Continued

\begin{tabular}{ccccc}
\hline \hline Cluster Name & GALEX IAU Name & 2MASS ID & IRAC AOR & MIPS AOR \\
\hline UGC 03957 & $074058.2+552538^{\mathrm{e}}$ & $07405822+5525379$ & $\ldots$ & $\ldots$ \\
UGC 12491 & $\ldots$ & $23183823+4257289$ & $\ldots$ & $\ldots$ \\
ZWCL 1215 & $121741.2+033922^{\mathrm{d}}$ & $12174112+0339208$ & $\ldots$ \\
ZWCL 1358+6245 & $135950.5+623104^{\mathrm{e}}$ & $13^{h} 59^{m} 50^{s} .0,62^{\circ} 31^{\prime} 03^{\prime \prime} 12657920,23473920^{\mathrm{j}}, 23474432^{\mathrm{j}}$ & $4770816^{\mathrm{a}}, 4771072^{\mathrm{a}}$ \\
ZWCL 1742 & $\ldots$ & $23478272^{\mathrm{k}}, 23478528^{\mathrm{k}}, 4754944$ & $18643456^{\mathrm{a}, b}$ \\
ZWCL 1953 & $\ldots$ & $17441450+3259292$ & 18656256 & $\ldots$ \\
ZWCL 3146 & $102339.6+041110^{\mathrm{e}}$ & $10233960+0411116$ & 12654592,4751872 & $4768256^{\mathrm{a}}, 4768512^{\mathrm{a}}, 4746496^{\mathrm{a}, b, f}$ \\
ZWICKY 2701 & $\ldots$ & $09524915+5153053$ & 18649856 & $18649600^{\mathrm{a}, b}$ \\
ZwCl 0857.9+2107 & $090036.9+205342^{\mathrm{e}}$ & $09003684+2053402$ & 18660096 & $18659840^{\mathrm{a}, b}$
\end{tabular}

${ }^{a}$ MIPS observation includes a $24 \mu \mathrm{m}$ observation.

${ }^{\mathrm{b}}$ MIPS observation includes a $70 \mu \mathrm{m}$ observation.

${ }^{\mathrm{c}} G A L E X$ observation only includes FUV measurement.

${ }^{\mathrm{d}}$ GALEX observation only includes NUV measurement.

${ }^{\mathrm{e}}$ GALEX observation includes both NUV and FUV measurement.

${ }^{\mathrm{f}}$ MIPS observation includes a $160 \mu \mathrm{m}$ observation.

${ }^{\mathrm{g}}$ Spitzer observations were made by Donahue as part of the DDT program 488 .

${ }^{\mathrm{h}}$ Due to an anomalous point source in the pbcd reduction, the images needed to be reduced from the bcd frames.

${ }^{\mathrm{i}}$ GALEX observation was taken as a Guest Investigator for Hicks et al. (2010).

${ }^{\mathrm{j} I R A C}$ observation only has bands 1 and 3 on target.

${ }^{\mathrm{k}}$ IRAC observation only has bands 2 and 4 on target. 
Table 3. Physical Properties.

\begin{tabular}{|c|c|c|c|c|c|c|c|c|}
\hline Cluster Name & Redshift & $\begin{array}{c}\text { Central Entropy } \\
\left(\mathrm{keV} \mathrm{cm}{ }^{2}\right)\end{array}$ & $\begin{array}{c}K_{100}{ }^{\mathrm{a}} \\
\left(\mathrm{keV} \mathrm{cm} \mathrm{cm}^{2}\right)\end{array}$ & alpha $^{\mathrm{a}}$ & $\begin{array}{c}\text { Scale } \\
\left(\mathrm{kpc} /{ }^{\prime \prime}\right)\end{array}$ & $\begin{array}{c}\text { IR Radius } \\
\left({ }^{\prime \prime}\right)\end{array}$ & $\begin{array}{c}\text { UV Radius } \\
\left({ }^{\prime \prime}\right)\end{array}$ & $\begin{array}{c}\text { Centroid Offset } \\
(\mathrm{kpc})\end{array}$ \\
\hline $1 \mathrm{E} 065756 \mathrm{a}$ & 0.2960 & 307.45 & 18.55 & 1.88 & 4.41 & 3.24 & $\cdots$ & 503.92 \\
\hline 1E0657 56b & 0.2960 & 307.45 & 18.55 & 1.88 & 4.41 & 3.24 & $\ldots$ & 216.72 \\
\hline 2A $0335+096$ & 0.0347 & 7.14 & 138.64 & 1.52 & 0.69 & 20.7 & 12.8 & 1.00 \\
\hline 2PIGG J0011.5-2850 & 0.0753 & 101.98 & 214.68 & 0.84 & 1.43 & 10.01 & 17.3 & 2.10 \\
\hline 2PIGG J2227.0-3041 & 0.0729 & 17.13 & 113.95 & 1.37 & 1.39 & 10.31 & 17.3 & 0.59 \\
\hline $3 \mathrm{C} 28.0$ & 0.1952 & 23.85 & 107.82 & 1.79 & 3.24 & 4.42 & 12.8 & 3.55 \\
\hline $3 \mathrm{C} 295$ & 0.4641 & 14.54 & 81.95 & 1.47 & 5.86 & 2.44 & 9 & 16.98 \\
\hline 3C 388 & 0.0917 & 17.03 & 214.3 & 0.76 & 1.71 & 8.38 & 17.3 & 1.44 \\
\hline $4 \mathrm{C} 55.16$ & 0.2420 & 23.27 & 161.6 & 1.29 & 3.82 & 3.75 & 9 & 31.64 \\
\hline Abell 13 & 0.0940 & 182.64 & 181.95 & 1.37 & 1.75 & 8.19 & 12.8 & 0.89 \\
\hline Abell 68 & 0.2546 & 217.34 & 142.31 & 0.89 & 3.96 & 3.61 & 9 & 3.77 \\
\hline Abell 85 & 0.0558 & 12.5 & 158.83 & 1.12 & 1.08 & 13.2 & 17.3 & 1.86 \\
\hline Abell 119 & 0.0442 & 233.85 & 191.29 & 0.75 & 0.87 & 16.44 & 17.3 & 2.20 \\
\hline Abell 133 & 0.0558 & 17.26 & 170.12 & 1.59 & 1.08 & 13.2 & 17.3 & 3.29 \\
\hline Abell 141 & 0.2300 & 205.03 & 42.56 & 1.78 & 3.67 & 3.89 & 9 & 4.38 \\
\hline Abell 160 & 0.0447 & 155.78 & 116.26 & 0.98 & 0.88 & 16.26 & 17.3 & 1.65 \\
\hline Abell 193 & 0.0485 & 185.46 & 35.96 & 2.23 & 0.95 & 15.05 & 17.3 & 0.57 \\
\hline Abell 209 & 0.2060 & 105.5 & 149.31 & 0.8 & 3.38 & 4.23 & 12.8 & 7.85 \\
\hline Abell 222 & 0.2130 & 126.02 & 82.25 & 1 & 3.47 & 4.13 & 9 & 7.83 \\
\hline Abell 223 & 0.2070 & 183.87 & 160.65 & 1.24 & 3.39 & 4.22 & 9 & 1.13 \\
\hline Abell 262 & 0.0164 & 10.57 & 205.09 & 0.98 & 0.33 & 42.84 & 17.3 & 0.25 \\
\hline Abell 267 & 0.2300 & 168.56 & 51.81 & 1.82 & 3.67 & 3.89 & 17.3 & 1.52 \\
\hline Abell 368 & 0.2200 & 50.87 & 144.12 & 1.21 & 3.55 & 4.03 & $\ldots$ & 60.21 \\
\hline Abell 370 & 0.3747 & 321.92 & 78.72 & 1.24 & 5.16 & 2.77 & 9 & 99.67 \\
\hline Abell 383 & 0.1871 & 13.02 & 110.9 & 1.4 & 3.13 & 4.57 & 17.3 & 2.63 \\
\hline Abell 399 & 0.0716 & 153.2 & 204.34 & 0.74 & 1.36 & 10.48 & 9 & 1.52 \\
\hline Abell 400 & 0.0240 & 162.81 & 35.26 & 1.76 & 0.48 & 29.55 & 17.3 & 4.61 \\
\hline Abell 401 & 0.0745 & 166.86 & 81.79 & 1.4 & 1.42 & 10.11 & 17.3 & 1.12 \\
\hline Abell 426 & 0.0179 & 19.38 & 119.89 & 1.74 & 0.36 & 39.32 & 17.3 & 0.33 \\
\hline Abell 478 & 0.0883 & 7.81 & 122 & 0.97 & 1.65 & 8.66 & $\ldots$ & 0.44 \\
\hline Abell 496 & 0.0328 & 8.91 & 216.33 & 1.27 & 0.65 & 21.85 & $\cdots$ & 1.48 \\
\hline Abell 520 & 0.2020 & 325.54 & 10.22 & 2.09 & 3.33 & 4.3 & $\cdots$ & 643.52 \\
\hline Abell 521 & 0.2533 & 259.87 & 245.43 & 1.91 & 3.95 & 3.62 & $\cdots$ & 4.23 \\
\hline Abell 539 & 0.0288 & 22.59 & 493.32 & 1.05 & 0.58 & 24.76 & 9 & 15.36 \\
\hline Abell 562 & 0.1100 & 202.06 & 34.61 & 1.09 & 2.01 & 7.13 & $\ldots$ & 0.43 \\
\hline Abell 576 & 0.0385 & 95.29 & 221.21 & 1.41 & 0.76 & 18.74 & 17.3 & 0.59 \\
\hline Abell 586 & 0.1710 & 94.75 & 92.06 & 1.25 & 2.91 & 4.91 & $\cdots$ & 2.51 \\
\hline Abell 611 & 0.2880 & 124.93 & 164.35 & 1.25 & 4.33 & 3.3 & 9 & 1.44 \\
\hline Abell $644^{\mathrm{b}}$ & 0.0698 & 132.36 & 85.91 & 1.55 & 1.33 & 10.73 & 7 & 0.97 \\
\hline Abell 665 & 0.1810 & 134.57 & 106.34 & 1.06 & 3.05 & 4.69 & 17.3 & 3.57 \\
\hline
\end{tabular}


Table 3-Continued

\begin{tabular}{|c|c|c|c|c|c|c|c|c|}
\hline Cluster Name & Redshift & $\begin{array}{c}\text { Central Entropy } \\
\left(\mathrm{keV} \mathrm{cm}{ }^{2}\right)\end{array}$ & $\begin{array}{c}K_{100}{ }^{\mathrm{a}} \\
\left(\mathrm{keV} \mathrm{cm}{ }^{2}\right)\end{array}$ & alpha $^{\mathrm{a}}$ & $\begin{array}{c}\text { Scale } \\
\left(\mathrm{kpc} /{ }^{\prime \prime}\right)\end{array}$ & $\begin{array}{c}\text { IR Radius } \\
\left({ }^{\prime \prime}\right)\end{array}$ & $\begin{array}{l}\text { UV Radius } \\
\left({ }^{\prime \prime}\right)\end{array}$ & $\begin{array}{c}\text { Centroid Offset } \\
(\mathrm{kpc})\end{array}$ \\
\hline Abell 697 & 0.2820 & 166.67 & 108.16 & 1.1 & 4.27 & 3.35 & 17.3 & 7.72 \\
\hline Abell 744 & 0.0729 & 63.42 & 229.33 & 0.79 & 1.39 & 10.31 & 17.3 & 0.79 \\
\hline Abell 754 & 0.0543 & 270.39 & 69.67 & 1.48 & 1.06 & 13.54 & 17.3 & 0.65 \\
\hline Abell 773 & 0.2170 & 244.32 & 41.1 & 1.6 & 3.52 & 4.07 & 9 & 7.22 \\
\hline Abell 907 & 0.1527 & 23.38 & 187.01 & 1.05 & 2.65 & 5.39 & 12.8 & 3.21 \\
\hline Abell 963 & 0.2056 & 55.77 & 169.12 & 0.9 & 3.37 & 4.24 & 17.3 & 6.41 \\
\hline Abell 1060 & 0.0125 & 72 & 178.34 & 1.25 & 0.26 & 55.94 & 17.3 & 0.35 \\
\hline Abell 1063S & 0.3540 & 169.59 & 42.21 & 1.72 & 4.98 & 2.87 & 9 & 4.31 \\
\hline Abell 1068 & 0.1375 & 9.11 & 108.8 & 1.31 & 2.43 & 5.88 & 17.3 & 1.74 \\
\hline Abell 1201 & 0.1688 & 64.81 & 198.94 & 1.03 & 2.88 & 4.96 & 17.3 & 1.62 \\
\hline Abell 1204 & 0.1706 & 15.31 & 81.83 & 1.4 & 2.91 & 4.92 & 9 & 4.68 \\
\hline Abell 1240 & 0.1590 & 462.4 & 8.27 & 2.37 & 2.74 & 5.21 & $\ldots$ & $\ldots$ \\
\hline Abell 1361 & 0.1171 & 18.64 & 117.86 & 1.06 & 2.12 & 6.75 & 17.3 & 8.10 \\
\hline Abell 1413 & 0.1426 & 64.03 & 123.19 & 1.19 & 2.51 & 5.71 & 17.3 & 2.90 \\
\hline Abell 1423 & 0.2130 & 68.32 & 124.17 & 1.2 & 3.47 & 4.13 & 9 & 2.14 \\
\hline Abell 1446 & 0.1035 & 152.38 & 119.5 & 0.67 & 1.9 & 7.52 & 12.8 & 3.11 \\
\hline Abell 1569 & 0.0735 & 110.1 & 149.14 & 0.51 & 1.4 & 10.23 & 9 & 0.43 \\
\hline Abell 1576 & 0.2790 & 186.24 & 98.35 & 1.38 & 4.23 & 3.38 & $\ldots$ & 13.26 \\
\hline Abell 1644 & 0.0471 & 19.03 & 585.67 & 1.76 & 0.92 & 15.48 & 17.3 & 0.54 \\
\hline Abell 1650 & 0.0843 & 37.96 & 159.89 & 0.84 & 1.58 & 9.03 & 17.3 & 1.09 \\
\hline Abell 1651 & 0.0840 & 89.46 & 115.53 & 0.97 & 1.58 & 9.06 & $\ldots$ & 1.95 \\
\hline Abell 1664 & 0.1276 & 14.4 & 141.84 & 1.7 & 2.28 & 6.27 & 12.8 & 1.12 \\
\hline Abell 1689 & 0.1843 & 78.44 & 111.77 & 1.35 & 3.09 & 4.62 & 9 & 21.14 \\
\hline Abell 1736 & 0.0338 & 150.35 & 127.25 & 0.99 & 0.67 & 21.23 & 12.8 & 0.20 \\
\hline Abell 1758 & 0.2792 & 230.84 & 144.04 & 1.21 & 4.24 & 3.38 & $\ldots$ & 272.85 \\
\hline Abell 1763 & 0.1866 & 214.69 & 70.8 & 1.37 & 3.12 & 4.58 & 17.3 & 0.91 \\
\hline Abell 1795 & 0.0625 & 18.99 & 130.38 & 1.18 & 1.2 & 11.88 & 17.3 & 1.21 \\
\hline Abell 1835 & 0.2532 & 11.44 & 111.71 & 1.26 & 3.95 & 3.62 & 12.8 & 7.93 \\
\hline Abell 1914 & 0.1712 & 107.16 & 131.15 & 1.05 & 2.91 & 4.91 & 9 & 58.40 \\
\hline Abell 1942 & 0.2240 & 107.73 & 194.08 & 0.66 & 3.6 & 3.97 & 9 & 3.00 \\
\hline Abell 1991 & 0.0587 & 1.53 & 152.21 & 1.09 & 1.14 & 12.59 & 17.3 & 1.90 \\
\hline Abell 1995 & 0.3186 & 374.35 & 26.81 & 2.08 & 4.64 & 3.08 & 9 & 2.89 \\
\hline Abell 2029 & 0.0765 & 10.5 & 163.58 & 0.95 & 1.45 & 9.86 & 17.3 & 1.75 \\
\hline Abell 2034 & 0.1130 & 232.64 & 85.07 & 1.14 & 2.05 & 6.96 & 17.3 & 3.94 \\
\hline Abell 2052 & 0.0353 & 9.45 & 164.72 & 1.25 & 0.7 & 20.36 & 17.3 & 0.80 \\
\hline Abell 2063 & 0.0351 & 53.51 & 129.01 & 1.07 & 0.7 & 20.47 & 17.3 & 1.11 \\
\hline Abell 2065 & 0.0730 & 43.95 & 195.29 & 1.02 & 1.39 & 10.29 & 9 & 112.52 \\
\hline Abell 2069 & 0.1160 & 453.25 & 54.57 & 1.47 & 2.1 & 6.81 & 9 & 2.26 \\
\hline Abell 2104 & 0.1554 & 160.61 & 210.1 & 1.2 & 2.69 & 5.31 & 9 & 0.96 \\
\hline Abell 2107 & 0.0411 & 21.24 & 396.13 & 0.91 & 0.81 & 17.61 & 17.3 & 0.91 \\
\hline
\end{tabular}


Table 3-Continued

\begin{tabular}{|c|c|c|c|c|c|c|c|c|}
\hline Cluster Name & Redshift & $\begin{array}{c}\text { Central Entropy } \\
\left(\mathrm{keV} \mathrm{cm}{ }^{2}\right)\end{array}$ & 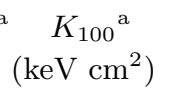 & alpha $^{a}$ & $\begin{array}{c}\text { Scale } \\
\left(\mathrm{kpc} /{ }^{\prime \prime}\right)\end{array}$ & $\begin{array}{l}\text { IR Radius } \\
\quad\left({ }^{\prime \prime}\right)\end{array}$ & $\begin{array}{l}\text { UV Radius } \\
\left({ }^{\prime \prime}\right)\end{array}$ & $\begin{array}{c}\text { Centroid Offset }^{\mathrm{a}} \\
(\mathrm{kpc})\end{array}$ \\
\hline Abell 2111 & 0.2300 & 107.36 & 193.98 & 0.65 & 3.67 & 3.89 & 17.3 & 4.20 \\
\hline Abell 2124 & 0.0658 & 98.31 & 260.81 & 0.9 & 1.26 & 11.33 & 17.3 & 1.19 \\
\hline Abell 2125 & 0.2465 & 225.17 & 32.9 & 1.35 & 3.87 & 3.7 & 9 & 18.90 \\
\hline Abell 2142 & 0.0898 & 68.06 & 120.57 & 1.22 & 1.68 & 8.53 & 17.3 & 0.93 \\
\hline Abell 2147 & 0.0356 & 151.86 & 136.19 & 0.55 & 0.71 & 20.2 & 17.3 & 0.28 \\
\hline Abell 2151 & 0.0366 & 0.37 & 135.18 & 0.56 & 0.73 & 19.67 & 17.3 & 0.12 \\
\hline Abell 2163 & 0.1695 & 437.98 & 72.21 & 1.87 & 2.89 & 4.95 & $\ldots$ & 524.17 \\
\hline Abell 2187 & 0.1833 & 78.63 & 170.71 & 0.96 & 3.08 & 4.64 & 12.8 & 0.53 \\
\hline Abell 2199 & 0.0300 & 13.27 & 331.46 & 1.35 & 0.6 & 23.81 & 17.3 & 0.42 \\
\hline Abell 2204 & 0.1524 & 9.74 & 166.19 & 1.41 & 2.65 & 5.4 & $\ldots$ & 21.70 \\
\hline Abell 2218 & 0.1713 & 288.56 & 10.72 & 2.35 & 2.92 & 4.9 & 9 & 26.65 \\
\hline Abell 2219 & 0.2256 & 411.57 & 17.01 & 1.97 & 3.62 & 3.95 & 17.3 & 0.54 \\
\hline Abell 2244 & 0.0967 & 57.58 & 109.07 & 1 & 1.79 & 7.99 & $\ldots$ & 3.45 \\
\hline Abell 2255 & 0.0805 & 529.1 & 5.78 & 2.63 & 1.52 & 9.42 & 12.8 & 149.58 \\
\hline Abell 2256 & 0.0579 & 349.58 & 7.05 & 2.54 & 1.12 & 12.75 & 12.8 & 877.60 \\
\hline Abell 2259 & 0.1640 & 113.98 & 60.96 & 1.36 & 2.81 & 5.08 & 9 & 3.42 \\
\hline Abell 2261 & 0.2240 & 61.08 & 105.96 & 1.27 & 3.6 & 3.97 & $\cdots$ & 1.82 \\
\hline Abell 2294 & 0.1780 & 156.31 & 235.74 & 1.03 & 3.01 & 4.75 & $\ldots$ & 9.62 \\
\hline Abell 2319 & 0.0562 & 270.23 & 39.42 & 1.76 & 1.09 & 13.11 & 12.8 & 6.12 \\
\hline Abell 2384 & 0.0945 & 38.48 & 139.21 & 1.49 & 1.75 & 8.15 & $\ldots$ & 3.69 \\
\hline Abell 2390 & 0.2301 & 14.73 & 202.92 & 1.07 & 3.68 & 3.89 & 17.3 & 3.02 \\
\hline Abell 2409 & 0.1479 & 73.81 & 120.76 & 0.97 & 2.58 & 5.53 & 9 & 5.60 \\
\hline Abell 2420 & 0.0846 & 332.56 & 64.33 & 1.12 & 1.59 & 9 & $\ldots$ & 1.22 \\
\hline Abell 2462 & 0.0737 & 129.68 & 83.2 & 0.77 & 1.4 & 10.21 & 12.8 & 0.17 \\
\hline Abell 2537 & 0.2950 & 110.41 & 124.72 & 1.26 & 4.4 & 3.25 & $\ldots$ & 3.60 \\
\hline Abell 2554 & 0.1103 & 105.09 & 318.36 & 0.66 & 2.01 & 7.11 & 12.8 & 1.59 \\
\hline Abell 2556 & 0.0862 & 12.38 & 115.76 & 1.13 & 1.62 & 8.85 & 17.3 & 0.93 \\
\hline Abell 2589 & 0.0415 & 51.99 & 109.56 & 0.61 & 0.82 & 17.45 & 17.3 & 0.66 \\
\hline Abell 2597 & 0.0854 & 10.6 & 98.88 & 1.26 & 1.6 & 8.93 & 17.3 & 2.16 \\
\hline Abell 2626 & 0.0573 & 23.19 & 144.08 & 1.05 & 1.11 & 12.88 & 17.3 & 3.36 \\
\hline Abell 2631 & 0.2779 & 308.81 & 29.18 & 1.44 & 4.22 & 3.39 & 17.3 & 4236.20 \\
\hline Abell 2657 & 0.0402 & 65.39 & 153.55 & 0.91 & 0.8 & 17.98 & 9 & 0.79 \\
\hline Abell 2667 & 0.2300 & 19.31 & 93.44 & 1.31 & 3.67 & 3.89 & 12.8 & 15.44 \\
\hline Abell 2717 & 0.0475 & 27.05 & 151.19 & 0.75 & 0.93 & 15.35 & 17.3 & 0.18 \\
\hline Abell 2744a & 0.3080 & 438.44 & 46.39 & 1.41 & 4.54 & 3.15 & $\ldots$ & 151.49 \\
\hline Abell 2744b & 0.3080 & 438.44 & 46.39 & 1.41 & 4.54 & 3.15 & $\cdots$ & 268.24 \\
\hline Abell 2813 & 0.2924 & 267.63 & 90.43 & 1.76 & 4.38 & 3.27 & 9 & 34.07 \\
\hline Abell 3084 & 0.0977 & 96.74 & 193.67 & 1.08 & 1.81 & 7.92 & 17.3 & 1.16 \\
\hline Abell 3088 & 0.2534 & 82.78 & 216.83 & 1.71 & 3.95 & 3.62 & $\ldots$ & 7.37 \\
\hline Abell 3112 & 0.0720 & 11.4 & 169.12 & 1.17 & 1.37 & 10.43 & 17.3 & 0.64 \\
\hline
\end{tabular}


Table 3-Continued

\begin{tabular}{|c|c|c|c|c|c|c|c|c|}
\hline Cluster Name & Redshift & $\begin{array}{c}\text { Central Entropy } \\
\left(\mathrm{keV} \mathrm{cm}{ }^{\mathrm{a}}\right)\end{array}$ & 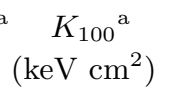 & alpha $^{\mathrm{a}}$ & $\begin{array}{c}\text { Scale } \\
\left(\mathrm{kpc} /{ }^{\prime \prime}\right)\end{array}$ & $\begin{array}{c}\text { IR Radius } \\
\quad\left({ }^{\prime \prime}\right)\end{array}$ & $\begin{array}{l}\text { UV Radius } \\
\left({ }^{\prime \prime}\right)\end{array}$ & $\begin{array}{c}\text { Centroid Offset }^{\mathrm{a}} \\
(\mathrm{kpc})\end{array}$ \\
\hline Abell 3120 & 0.0690 & 17.29 & 206.17 & 0.99 & 1.32 & 10.84 & 17.3 & 0.48 \\
\hline Abell 3158 & 0.0580 & 166.01 & 80.92 & 0.9 & 1.12 & 12.73 & 17.3 & 1.51 \\
\hline Abell 3266 & 0.0590 & 72.45 & 376.73 & 0.64 & 1.14 & 12.53 & 17.3 & 7.23 \\
\hline Abell 3364 & 0.1483 & 268.55 & 34.53 & 1.97 & 2.59 & 5.52 & $\cdots$ & 1.26 \\
\hline Abell 3376 & 0.0456 & 282.95 & 58.97 & 1.71 & 0.9 & 15.96 & 17.3 & 0.99 \\
\hline Abell 3391 & 0.0560 & 367.52 & 23.56 & 1.64 & 1.09 & 13.15 & $\cdots$ & 1.31 \\
\hline Abell 3395 & 0.0510 & 247.2 & 105.85 & 1.65 & 1 & 14.36 & $\ldots$ & 2.20 \\
\hline Abell 3528S & 0.0530 & 31.56 & 270.04 & 1.17 & 1.03 & 13.85 & 17.3 & 2.05 \\
\hline Abell 3558 & 0.0480 & 126.15 & 132.52 & 2.11 & 0.94 & 15.2 & 17.3 & 0.38 \\
\hline Abell 3562 & 0.0490 & 77.42 & 159.76 & 0.81 & 0.96 & 14.91 & 12.8 & 0.93 \\
\hline Abell 3571 & 0.0391 & 79.31 & 191.27 & 0.82 & 0.77 & 18.47 & $\cdots$ & 0.53 \\
\hline Abell 3581 & 0.0218 & 9.51 & 138.07 & 1.22 & 0.44 & 32.44 & 17.3 & 0.70 \\
\hline Abell 3667 & 0.0556 & 160.43 & 110.56 & 0.78 & 1.08 & 13.24 & $\ldots$ & 1.32 \\
\hline Abell 3822 & 0.0759 & 108.66 & 200.32 & 0.66 & 1.44 & 9.93 & 17.3 & 0.87 \\
\hline Abell 3827 & 0.0984 & 164.58 & 94.8 & 1.34 & 1.82 & 7.87 & 17.3 & 2.96 \\
\hline Abell 3921 & 0.0927 & 101.19 & 151.49 & 0.86 & 1.72 & 8.29 & 17.3 & 1.33 \\
\hline Abell 4038 & 0.0300 & 37.93 & 117.86 & 1.11 & 0.6 & 23.81 & $\cdots$ & 0.58 \\
\hline Abell 4059 & 0.0475 & 7.06 & 203.16 & 0.88 & 0.93 & 15.35 & 17.3 & 1.40 \\
\hline Abell S0405 & 0.0613 & 16.94 & 274.22 & 0.45 & 1.18 & 12.09 & 17.3 & 5.47 \\
\hline Abell S0592 & 0.2216 & 58.7 & 195.48 & 0.99 & 3.57 & 4 & $\cdots$ & 6.93 \\
\hline AC 114 & 0.3120 & 199.79 & 69.99 & 1.5 & 4.58 & 3.13 & 9 & 2.48 \\
\hline AWM7 & 0.0172 & 8.37 & 227.59 & 0.8 & 0.35 & 40.89 & 17.3 & 0.26 \\
\hline CENTAURUS & 0.0109 & 2.25 & 474.86 & 1.33 & 0.22 & 64.03 & 17.3 & 0.08 \\
\hline CID 0072 & 0.0344 & 9.45 & 133.25 & 0.99 & 0.69 & 20.87 & 17.3 & 0.60 \\
\hline CL J1226.9+3332 & 0.8900 & 166.03 & 99 & 1.41 & 7.77 & 1.84 & 7 & 15.14 \\
\hline CYGNUS A & 0.0561 & 23.64 & 210.12 & 1.57 & 1.09 & 13.13 & $\cdots$ & 0.85 \\
\hline ESO 3060170 & 0.0358 & 7.98 & 1400.88 & 1.8 & 0.71 & 20.09 & 17.3 & 1.03 \\
\hline ESO 5520200 & 0.0314 & 5.89 & 121.77 & 0.67 & 0.63 & 22.78 & 17.3 & 0.61 \\
\hline EXO 0422-086 & 0.0397 & 13.77 & 193.82 & 1.25 & 0.79 & 18.2 & 9 & 2.07 \\
\hline HCG 0062 & 0.0146 & 3.44 & 219.02 & 1.28 & 0.3 & 48.02 & 17.3 & 7.71 \\
\hline HCG 42 & 0.0133 & 1.85 & 126.46 & 0.87 & 0.27 & 52.63 & 17.3 & 0.49 \\
\hline HERCULES A & 0.1541 & 9.17 & 143.89 & 1.07 & 2.67 & 5.35 & 17.3 & 8.65 \\
\hline HYDRA A & 0.0549 & 13.31 & 114.92 & 1.03 & 1.07 & 13.4 & 17.3 & 0.40 \\
\hline M49 & 0.0033 & 0.91 & 495.33 & 1.14 & 0.07 & 209.55 & 17.3 & 0.07 \\
\hline M87 & 0.0044 & 3.53 & 146.6 & 0.8 & 0.09 & 157.37 & 17.3 & 0.09 \\
\hline MACS J0011.7-1523 & 0.3600 & 18.83 & 109.14 & 1.04 & 5.03 & 2.84 & $\cdots$ & $\cdots$ \\
\hline MACS J0035.4-2015 & 0.3644 & 93.42 & 76.4 & 1.26 & 5.07 & 2.82 & $\cdots$ & 248.81 \\
\hline MACS J0159.8-0849 & 0.4050 & 18.79 & 123.87 & 1.31 & 5.41 & 2.64 & $\cdots$ & $\ldots$ \\
\hline MACS J0242.5-2132 & 0.3140 & 10.92 & 74.59 & 1.29 & 4.6 & 3.11 & $\cdots$ & $\cdots$ \\
\hline MACS J0257.1-2325 & 0.5053 & 234.47 & 195.81 & 1.39 & 6.14 & 2.33 & $\cdots$ & $\ldots$ \\
\hline
\end{tabular}


Table 3-Continued

\begin{tabular}{|c|c|c|c|c|c|c|c|c|}
\hline Cluster Name & Redshift & $\begin{array}{l}\text { Central Entropy } \\
\quad\left(\mathrm{keV} \mathrm{cm}^{2}\right)\end{array}$ & $\begin{array}{c}K_{100}{ }^{\mathrm{a}} \\
\left(\mathrm{keV} \mathrm{cm}^{2}\right)\end{array}$ & $\operatorname{alpha}^{a}$ & $\begin{array}{c}\text { Scale } \\
\left(\mathrm{kpc} / /^{\prime \prime}\right)\end{array}$ & $\begin{array}{c}\text { IR Radius } \\
\left({ }^{\prime \prime}\right)\end{array}$ & $\begin{array}{l}\text { UV Radius } \\
\left({ }^{\prime \prime}\right)\end{array}$ & $\begin{array}{c}\text { Centroid Offset }^{\mathrm{a}} \\
(\mathrm{kpc})\end{array}$ \\
\hline MACS J0257.6-2209 & 0.3224 & 155.91 & 82.11 & 1.55 & 4.68 & 3.06 & 9 & 1.52 \\
\hline MACS J0308.9+2645 & 0.3240 & 212.76 & 70.07 & 1.43 & 4.69 & 3.05 & 9 & 4.91 \\
\hline MACS J0329.6-0211 & 0.4500 & 11.13 & 96.68 & 1.26 & 5.76 & 2.48 & $\cdots$ & 7.08 \\
\hline MACS J0417.5-1154 & 0.4400 & 27.08 & 99.69 & 1.42 & 5.69 & 2.52 & $\cdots$ & $\cdots$ \\
\hline MACS J0429.6-0253 & 0.3990 & 17.17 & 88.87 & 1.23 & 5.36 & 2.67 & $\cdots$ & 1.91 \\
\hline MACS J0520.7-1328 & 0.3398 & 88.59 & 84.92 & 1.2 & 4.85 & 2.95 & $\cdots$ & 8.14 \\
\hline MACS J0547.0-3904 & 0.2100 & 23.14 & 121.59 & 1.2 & 3.43 & 4.17 & $\cdots$ & $\cdots$ \\
\hline MACS J0717.5+3745 & 0.5480 & 220.15 & 160.08 & 0.76 & 6.4 & 2.23 & $\cdots$ & $\cdots$ \\
\hline MACS J0744.8+3927 & 0.6860 & 42.38 & 112.02 & 1.11 & 7.09 & 2.02 & $\cdots$ & 101.36 \\
\hline MACS J1115.2+5320 & 0.4390 & 292.28 & 27.56 & 1.73 & 5.68 & 2.52 & $\cdots$ & $\ldots$ \\
\hline MACS J1115.8+0129 & 0.3521 & 14.76 & 107.65 & 1.31 & 4.96 & 2.88 & $\cdots$ & $\cdots$ \\
\hline MACS J1131.8-1955 & 0.3070 & 97.26 & 156.32 & 1.15 & 4.53 & 3.16 & $\cdots$ & 2.56 \\
\hline MACS J1149.5+2223 & 0.5440 & 280.69 & 33.13 & 1.47 & 6.38 & 2.24 & $\cdots$ & 18.05 \\
\hline MACS J1206.2-0847 & 0.4400 & 69.04 & 94.69 & 1.28 & 5.69 & 2.52 & $\cdots$ & $\ldots$ \\
\hline MACS J1311.0-0310 & 0.4940 & 47.36 & 63.47 & 1.62 & 6.06 & 2.36 & $\cdots$ & $\cdots$ \\
\hline MACS J1621.3+3810 & 0.4610 & 20.08 & 129.83 & 1.18 & 5.84 & 2.45 & $\cdots$ & $\cdots$ \\
\hline MACS J1931.8-2634 & 0.3520 & 14.56 & 87.52 & 1.27 & 4.96 & 2.88 & $\cdots$ & $\cdots$ \\
\hline MACS J2049.9-3217 & 0.3254 & 195.85 & 92.71 & 1.06 & 4.71 & 3.04 & $\cdots$ & $\cdots$ \\
\hline MACS J2211.7-0349 & 0.2700 & 165.49 & 78.26 & 1.59 & 4.14 & 3.46 & $\cdots$ & 2.55 \\
\hline MACS J2214.9-1359 & 0.5026 & 297.7 & 171.96 & 1.46 & 6.12 & 2.34 & $\cdots$ & 98.06 \\
\hline MACS J2228+2036 & 0.4120 & 118.79 & 107.22 & 1 & 5.47 & 2.61 & $\cdots$ & $\cdots$ \\
\hline MACS J2229.7-2755 & 0.3240 & 12.39 & 74.97 & 1.36 & 4.69 & 3.05 & 17.3 & 6.04 \\
\hline MACS J2245.0+2637 & 0.3040 & 42.03 & 105.93 & 1.33 & 4.5 & 3.18 & 9 & 2.65 \\
\hline MKW3S & 0.0450 & 23.94 & 131.08 & 0.96 & 0.88 & 16.16 & 17.3 & 0.78 \\
\hline MKW 04 & 0.0198 & 6.86 & 392.7 & 1.26 & 0.4 & 35.63 & 17.3 & 0.21 \\
\hline MKW 08 & 0.0270 & 130.68 & 228.51 & 0.87 & 0.54 & 26.36 & 17.3 & 32.83 \\
\hline MS 0016.9+1609 & 0.5410 & 162.07 & 64.17 & 1.29 & 6.36 & 2.25 & $\cdots$ & $\ldots$ \\
\hline MS 0116.3-0115 & 0.0452 & 12.77 & 220.8 & 0.63 & 0.89 & 16.09 & 17.3 & 0.64 \\
\hline MS 0440.5+0204 & 0.1900 & 25.46 & 164.03 & 1.11 & 3.17 & 4.51 & 9 & 1.11 \\
\hline MS 0451.6-0305 & 0.5386 & 568.13 & 15.61 & 2.81 & 6.34 & 2.25 & $\cdots$ & 14.34 \\
\hline MS 0735.6+7421 & 0.2160 & 15.96 & 106.79 & 1.14 & 3.5 & 4.08 & 9 & 2.85 \\
\hline MS 0839.8+2938 & 0.1940 & 19.19 & 105.79 & 1.33 & 3.22 & 4.44 & 9 & 0.83 \\
\hline MS 0906.5+1110 & 0.1630 & 104.23 & 97.32 & 1.15 & 2.8 & 5.11 & $\cdots$ & 1.49 \\
\hline MS $1006.0+1202$ & 0.2210 & 160.28 & 82.81 & 1.32 & 3.56 & 4.01 & $\cdots$ & 8.20 \\
\hline MS 1008.1-1224 & 0.3010 & 97.62 & 262.05 & 0.76 & 4.46 & 3.2 & $\cdots$ & 2.19 \\
\hline MS $1455.0+2232$ & 0.2590 & 16.88 & 81.47 & 1.39 & 4.01 & 3.56 & 9 & 5.70 \\
\hline MS 2137.3-2353 & 0.3130 & 14.68 & 89.85 & 1.39 & 4.59 & 3.12 & 17.3 & 4.20 \\
\hline MS J1157.3+5531 & 0.0810 & 5.93 & 277.01 & 1.45 & 1.53 & 9.36 & 9 & 3.38 \\
\hline NGC 0507 & 0.0164 & 0 & 99.89 & 0.65 & 0.33 & 42.84 & 17.3 & 0.27 \\
\hline NGC 4636 & 0.0031 & 1.42 & 11962.07 & 1.96 & 0.06 & 223.01 & 17.3 & 0.01 \\
\hline
\end{tabular}


Table 3-Continued

\begin{tabular}{|c|c|c|c|c|c|c|c|c|}
\hline Cluster Name & Redshift & $\begin{array}{c}\text { Central Entropy } \\
\left(\mathrm{keV} \mathrm{cm}{ }^{\mathrm{a}}\right)\end{array}$ & $\begin{array}{c}K_{100}{ }^{\mathrm{a}} \\
\left(\mathrm{keV} \mathrm{cm}^{2}\right)\end{array}$ & alpha $^{\mathrm{a}}$ & $\begin{array}{l}\text { Scale } \\
\left(\mathrm{kpc} /{ }^{\prime \prime}\right)\end{array}$ & $\begin{array}{c}\text { IR Radius } \\
\left({ }^{\prime \prime}\right)\end{array}$ & $\begin{array}{c}\text { UV Radius } \\
\left({ }^{\prime \prime}\right)\end{array}$ & $\begin{array}{c}\text { Centroid Offset }^{\mathrm{a}} \\
(\mathrm{kpc})\end{array}$ \\
\hline NGC 5044 & 0.0090 & 2.28 & 82.15 & 0.96 & 0.18 & 77.37 & 17.3 & 0.06 \\
\hline NGC 5813 & 0.0066 & 1.38 & 102.51 & 0.91 & 0.14 & 105.2 & 17.3 & 0.03 \\
\hline NGC 5846 & 0.0057 & 1.83 & 685.78 & 1.44 & 0.12 & 121.67 & 17.3 & 0.07 \\
\hline OPHIUCHUS & 0.0280 & 8.95 & 247.48 & 0.73 & 0.56 & 25.45 & 12.8 & 2.28 \\
\hline PKS 0745-191 & 0.1028 & 12.41 & 110.7 & 1.39 & 1.89 & 7.57 & $\ldots$ & 8.00 \\
\hline RBS 0461 & 0.0290 & 95.67 & 68.8 & 1.39 & 0.58 & 24.6 & 9 & 0.34 \\
\hline RBS 0533 & 0.0123 & 2.22 & 164.34 & 1 & 0.25 & 56.84 & 17.3 & 0.33 \\
\hline RBS 0797 & 0.3540 & 19.49 & 97.67 & 1.67 & 4.98 & 2.87 & 9 & 3.38 \\
\hline RCS J2327-0204 & 0.2000 & 68.55 & 217.2 & 1.28 & 3.3 & 4.33 & 17.3 & 261.00 \\
\hline RXCJ0331.1-2100 & 0.1880 & 11.4 & 134.1 & 1.3 & 3.14 & 4.55 & $\ldots$ & 1.09 \\
\hline RXC J1023.8-2715 & 0.0963 & 20.86 & 255.29 & 1.45 & 1.78 & 8.02 & 12.8 & 1.03 \\
\hline RX J0220.9-3829 & 0.2287 & 43 & 159.95 & 1.23 & 3.66 & 3.91 & $\cdots$ & 1.44 \\
\hline RX J0232.2-4420 & 0.2836 & 44.62 & 166.53 & 1.16 & 4.28 & 3.34 & $\cdots$ & $\ldots$ \\
\hline RX J0439+0520 & 0.2080 & 14.92 & 95.47 & 1.19 & 3.4 & 4.2 & 9 & 4.10 \\
\hline RX J0439.0+0715 & 0.2300 & 66.75 & 129.57 & 1.06 & 3.67 & 3.89 & $\cdots$ & 4.00 \\
\hline RX J0528.9-3927 & 0.2632 & 72.85 & 99.8 & 1.47 & 4.06 & 3.52 & $\ldots$ & $\ldots$ \\
\hline RX J0647.7+7015 & 0.5840 & 225.06 & 48.82 & 1.7 & 6.6 & 2.17 & $\cdots$ & 108.26 \\
\hline RX J0819.6+6336 & 0.1190 & 20.69 & 170.57 & 0.68 & 2.15 & 6.66 & 9 & 3.69 \\
\hline RX J1000.4+4409 & 0.1540 & 27.71 & 151.1 & 1.09 & 2.67 & 5.35 & 9 & 30.73 \\
\hline RX J1022.1+3830 & 0.0491 & 51.6 & 194.76 & 1.04 & 0.96 & 14.88 & 17.3 & 18.72 \\
\hline RX J1130.0+3637 & 0.0600 & 29.93 & 149.6 & 1.14 & 1.16 & 12.34 & 17.3 & 0.92 \\
\hline RX J1320.2+3308 & 0.0366 & 8.8 & 140.28 & 1.28 & 0.73 & 19.67 & 17.3 & 0.85 \\
\hline RX J1347.5-1145 & 0.4510 & 12.45 & 179.86 & 1.06 & 5.77 & 2.48 & 9 & 34.95 \\
\hline RX J1423.8+2404 & 0.5450 & 10.23 & 119.91 & 1.27 & 6.38 & 2.24 & $\ldots$ & $\ldots$ \\
\hline RX J1504.1-0248 & 0.2150 & 13.08 & 95.58 & 1.5 & 3.49 & 4.1 & 12.8 & 2.93 \\
\hline RX J1532.9+3021 & 0.3450 & 16.93 & 76.31 & 1.51 & 4.89 & 2.92 & $\ldots$ & $\cdots$ \\
\hline RX J1539.5-8335 & 0.0728 & 25.88 & 109.99 & 1.41 & 1.39 & 10.32 & $\ldots$ & 4.41 \\
\hline RX J1720.1+2638 & 0.1640 & 21.03 & 109.14 & 1.39 & 2.81 & 5.08 & 17.3 & 1.17 \\
\hline RX J1720.2+3536 & 0.3913 & 24 & 94.44 & 1.42 & 5.3 & 2.7 & $\cdots$ & $\cdots$ \\
\hline RX J1852.1+5711 & 0.1094 & 18.72 & 170.36 & 0.83 & 2 & 7.16 & 9 & 0.99 \\
\hline RX J2129.6+0005 & 0.2350 & 21.14 & 97.91 & 1.26 & 3.73 & 3.83 & 17.3 & 0.61 \\
\hline SC 1327-312 & 0.0531 & 64.57 & 160.82 & 0.81 & 1.03 & 13.83 & 17.3 & 0.48 \\
\hline SERSIC 159-03 & 0.0580 & 10.45 & 77.78 & 1.17 & 1.12 & 12.73 & 17.3 & 0.85 \\
\hline SS2B153 & 0.0186 & 1.12 & 71.35 & 0.8 & 0.38 & 37.88 & 17.3 & 0.66 \\
\hline UGC 03957 & 0.0341 & 12.88 & 175.07 & 0.98 & 0.68 & 21.05 & 17.3 & 0.27 \\
\hline UGC 12491 & 0.0174 & 2.99 & 148.48 & 1.12 & 0.35 & 40.43 & $\ldots$ & 0.43 \\
\hline ZWCL 1215 & 0.0750 & 163.23 & 131.29 & 1 & 1.42 & 10.04 & 9 & 3.09 \\
\hline ZWCL $1358+6245$ & 0.3280 & 20.67 & 97.97 & 1.43 & 4.73 & 3.02 & 9 & 18.84 \\
\hline ZWCL 1742 & 0.0757 & 23.84 & 126.53 & 1.3 & 1.44 & 9.96 & $\cdots$ & 0.76 \\
\hline ZWCL 1953 & 0.3800 & 194.53 & 62.12 & 1.39 & 5.21 & 2.75 & $\ldots$ & 4.25 \\
\hline
\end{tabular}


Table 3-Continued

\begin{tabular}{|c|c|c|c|c|c|c|c|c|}
\hline Cluster Name & Redshift & $\begin{array}{l}\text { Central Entropy } \\
\quad\left(\mathrm{keV} \mathrm{cm}{ }^{2}\right)\end{array}$ & $\begin{array}{c}K_{100}{ }^{\mathrm{a}} \\
\left(\mathrm{keV} \mathrm{cm}{ }^{2}\right)\end{array}$ & alpha $^{a}$ & $\begin{array}{l}\text { Scale } \\
\left(\mathrm{kpc} /{ }^{\prime \prime}\right)\end{array}$ & $\begin{array}{l}\text { IR Radius } \\
\left(^{\prime \prime}\right)\end{array}$ & $\begin{array}{l}\text { UV Radius } \\
\left({ }^{\prime \prime}\right)\end{array}$ & $\begin{array}{l}\text { Centroid Offset }{ }^{\mathrm{a}} \\
(\mathrm{kpc})\end{array}$ \\
\hline ZWCL 3146 & 0.2900 & 11.42 & 105.48 & 1.29 & 4.35 & 3.29 & 12.8 & 2.39 \\
\hline ZWICKY 2701 & 0.2100 & 39.66 & 126 & 1.45 & 3.43 & 4.17 & 9 & 1.71 \\
\hline $\mathrm{ZwCl} 0857.9+2107$ & 0.2350 & 24.25 & 89.27 & 1.4 & 3.73 & 3.83 & 17.3 & 3.81 \\
\hline
\end{tabular}

${ }^{a}$ Quantities defined in Cavagnolo et al. (2009) are from radially fit entropy profiles with a functional form $\mathrm{K}(\mathrm{r})=\mathrm{K}_{0}+\mathrm{K}_{\mathrm{x}}\left(\mathrm{r} / \mathrm{r}_{\mathrm{x}}\right)^{\alpha}$, where $\mathrm{K}_{0}$ is the central entropy in excess above the power law fit, $\mathrm{K}_{100}$ is the entropy profile normalization at $100 h_{70}^{-1} \mathrm{kpc}$ from the X-ray centroid, and alpha is the best-fit power law index.

${ }^{\mathrm{b}} \mathrm{UV}$ data is taken from Hicks et al. (2010)

Note. - Redshift information and central entropies are reproduced from the ACCEPT database. The size scale is calculated as the angular distance size assuming the standard cosmology in the paper. The IR radii are set at $14.3 h_{70}^{-1}$ $\mathrm{kpc}$ in size and are used for $2 M A S S$ and IRAC aperture measurements except in the case where the aperture is below $5^{\prime \prime}$. In this case the aperture is set to be $5^{\prime \prime}$ to minimize large aperture corrections. The UV radius is set by the aperture photometry in the GALEX database which most closely matches the GALEXView total flux measurement. We compare the BCG positions that we derive from the 2MASS locations in Table 2 and compare that to the X-ray centroid in the ACCEPT database. These distances are presented in a histogram in Figure 1 
Table 4. Fluxes Matched to UV Aperture.

\begin{tabular}{|c|c|c|c|c|c|c|c|c|c|c|}
\hline $\begin{array}{c}\text { Name } \\
\text { Cluster Name }\end{array}$ & $\begin{array}{l}\text { Flux } \\
\text { NUV } \\
\text { (mag) }\end{array}$ & $\begin{array}{l}\text { Error } \\
\text { NUV } \\
\text { (mag) }\end{array}$ & $\begin{array}{l}\text { Flux } \\
\text { FUV } \\
\text { (mag) }\end{array}$ & $\begin{array}{l}\text { Error } \\
\text { FUV } \\
\text { (mag) }\end{array}$ & $\begin{array}{c}\text { Flux } \\
\text { J } \\
(\mathrm{mag})\end{array}$ & $\begin{array}{c}\text { Error } \\
\mathrm{J} \\
(\mathrm{mag})\end{array}$ & $\begin{array}{c}\text { Flux } \\
\text { H } \\
(\mathrm{mag})\end{array}$ & $\begin{array}{c}\text { Error } \\
\text { H } \\
(\mathrm{mag})\end{array}$ & $\begin{array}{c}\text { Flux } \\
\text { K } \\
(\mathrm{mag})\end{array}$ & $\begin{array}{c}\text { Error } \\
\mathrm{K} \\
(\mathrm{mag})\end{array}$ \\
\hline $1 \mathrm{E} 065756 \mathrm{a}$ & $\cdots$ & $\cdots$ & $\cdots$ & $\cdots$ & $\cdots$ & $\cdots$ & $\cdots$ & $\cdots$ & $\cdots$ & $\cdots$ \\
\hline $1 \mathrm{E} 065756 \mathrm{~b}$ & $\ldots$ & $\ldots$ & $\ldots$ & $\ldots$ & $\ldots$ & $\ldots$ & $\ldots$ & $\ldots$ & $\ldots$ & $\ldots$ \\
\hline 2A $0335+096$ & 18.05 & 0 & 18.50 & 0 & 13.48 & 0.0130 & 13.13 & 0.0160 & 13.09 & 0.0160 \\
\hline 2PIGG J0011.5-2850 & 20.07 & 0.1093 & 20.65 & 0.2108 & 13.15 & 0.0080 & 12.90 & 0.0100 & 13.05 & 0.0131 \\
\hline 2PIGG J2227.0-3041 & 18.40 & 0.0176 & 19.85 & 0.0568 & 12.98 & 0.0070 & 12.75 & 0.0090 & 12.90 & 0.0106 \\
\hline $3 \mathrm{C} 28.0$ & 20.51 & 0.3453 & 20.56 & 0.2914 & 15.54 & 0.0660 & 15.35 & 0.0720 & 15.11 & 0.0697 \\
\hline $3 \mathrm{C} 295$ & 23.17 & 0 & 23.80 & 0.3245 & 16.38 & 0.1570 & 16.18 & 0.2230 & 15.76 & 0.1470 \\
\hline 3C 388 & 19.95 & 0.0507 & 20.97 & 0.1878 & 13.29 & 0.0100 & 13.10 & 0.0120 & 13.28 & 0.0139 \\
\hline $4 \mathrm{C} 55.16$ & 21.49 & 0 & 21.09 & 0 & 16.28 & 0.1640 & 15.93 & 0.2000 & 15.86 & 0.1770 \\
\hline Abell 13 & 21.82 & 0 & 21.37 & 0 & 14.73 & 0.0370 & 14.41 & 0.0460 & 14.48 & 0.0510 \\
\hline Abell 68 & 20.60 & 0 & 20.31 & 0 & 15.86 & 0.1240 & 15.91 & 0.2080 & 15.31 & 0.1310 \\
\hline Abell $85^{\mathrm{a}}$ & 18.85 & 0.0165 & 19.79 & 0.0323 & 12.50 & 0.0050 & 12.23 & 0.0060 & 12.41 & 0.0083 \\
\hline Abell 119 & 19.40 & 0.0289 & 20.05 & 0.0482 & 12.44 & 0.0041 & 12.21 & 0.0050 & 12.37 & 0.0070 \\
\hline Abell 133 & 19.28 & 0.1079 & 20.16 & 0.2425 & 12.81 & 0.0070 & 12.61 & 0.0110 & 12.69 & 0.0105 \\
\hline Abell 141 & 21.38 & 0 & 20.92 & 0 & 16.46 & 0.1770 & 15.84 & 0.1500 & 15.95 & 0.1950 \\
\hline Abell 160 & 19.24 & 0.0591 & 20.37 & 0 & 12.75 & 0.0070 & 12.60 & 0.0080 & 12.79 & 0.0131 \\
\hline Abell 193 & 21.05 & 0 & 20.39 & 0.2598 & 12.95 & 0.0070 & 12.68 & 0.0080 & 12.80 & 0.0110 \\
\hline Abell 209 & 21.64 & 0 & 21.89 & 0.0703 & 15.37 & 0.0690 & 15.26 & 0.1000 & 15.00 & 0.0840 \\
\hline Abell 222 & 21.59 & 0 & 21.13 & 0 & 15.79 & 0.0910 & 15.72 & 0.1410 & 15.49 & 0.1330 \\
\hline Abell 223 & 21.54 & 0 & 21.09 & 0 & 16.13 & 0.1260 & 15.79 & 0.1550 & 15.44 & 0.1290 \\
\hline Abell 262 & 18.21 & 0.0275 & 19.48 & 0.0714 & 11.43 & 0.0020 & 11.20 & 0.0030 & 11.53 & 0.0027 \\
\hline Abell 267 & 21.21 & 0.2633 & 21.60 & 0 & 14.90 & 0.0380 & 14.52 & 0.0510 & 14.47 & 0.0404 \\
\hline Abell 368 & $\ldots$ & $\ldots$ & $\ldots$ & $\ldots$ & $\ldots$ & $\ldots$ & $\ldots$ & $\ldots$ & $\ldots$ & $\ldots$ \\
\hline Abell 370 & 21.71 & 0 & 21.29 & 0 & 16.27 & 0.1480 & 16.12 & 0.1720 & 15.64 & 0.1550 \\
\hline Abell 383 & 20.25 & 0.2779 & 18.86 & 0.1555 & 14.61 & 0.0310 & 14.42 & 0.0400 & 14.16 & 0.0386 \\
\hline Abell 399 & 20.38 & 0 & 20.27 & 0 & 14.18 & 0.0240 & 13.92 & 0.0310 & 13.84 & 0.0310 \\
\hline Abell 400 & 18.60 & 0.1675 & 20.00 & 0 & 11.39 & 0.0030 & 11.25 & 0.0030 & 11.64 & 0.0034 \\
\hline Abell 401 & 19.53 & 0.3106 & 20.33 & 0 & 12.79 & 0.0090 & 12.60 & 0.0100 & 12.85 & 0.0113 \\
\hline Abell 426 & 14.23 & 0.0013 & 15.33 & 0.0029 & 10.78 & 0.0300 & 10.68 & 0.0300 & 11.03 & 0.0300 \\
\hline Abell 478 & $\ldots$ & $\ldots$ & $\ldots$ & $\ldots$ & $\ldots$ & $\ldots$ & $\ldots$ & $\ldots$ & $\ldots$ & $\ldots$ \\
\hline Abell 496 & $\ldots$ & $\ldots$ & $\ldots$ & $\ldots$ & $\ldots$ & $\ldots$ & $\ldots$ & $\ldots$ & $\ldots$ & $\ldots$ \\
\hline Abell 520 & $\ldots$ & $\ldots$ & $\ldots$ & $\ldots$ & $\ldots$ & $\ldots$ & $\ldots$ & $\ldots$ & $\ldots$ & $\ldots$ \\
\hline Abell 521 & $\ldots$ & $\ldots$ & $\ldots$ & $\ldots$ & $\ldots$ & $\ldots$ & $\ldots$ & $\ldots$ & $\ldots$ & $\ldots$ \\
\hline Abell 539 & 18.82 & 0.2203 & 20.07 & 0 & 12.85 & 0.0050 & 12.74 & 0.0060 & 13.04 & 0.0064 \\
\hline Abell 562 & $\ldots$ & $\ldots$ & $\ldots$ & $\ldots$ & $\ldots$ & $\ldots$ & $\ldots$ & $\ldots$ & $\ldots$ & $\ldots$ \\
\hline Abell 576 & 21.25 & 0 & 20.92 & 0 & 13.56 & 0.0130 & 13.28 & 0.0160 & 13.38 & 0.0180 \\
\hline Abell 586 & $\ldots$ & $\ldots$ & $\ldots$ & $\ldots$ & $\ldots$ & $\ldots$ & $\ldots$ & $\ldots$ & $\ldots$ & $\ldots$ \\
\hline Abell 611 & 22.57 & 0 & 22.65 & 0.3308 & 16.11 & 0.1510 & 15.46 & 0.1270 & 15.47 & 0.1200 \\
\hline Abell $644^{\mathrm{a}} \mathrm{b}$ & 20.94 & 0.08 & 20.39 & 0 & 13.92 & 0.0260 & 13.77 & 0.0300 & 14.02 & 0.0430 \\
\hline Abell 665 & 22.71 & 0 & 21.64 & 0.3204 & 15.44 & 0.0730 & 15.10 & 0.0930 & 15.26 & 0.1020 \\
\hline Abell 697 & 22.02 & 0.1797 & 22.98 & 0.2221 & 14.71 & 0.0470 & 14.73 & 0.0590 & 14.57 & 0.0476 \\
\hline Abell 744 & 20.17 & 0.2177 & 21.01 & 0 & 13.44 & 0.0110 & 13.18 & 0.0140 & 13.36 & 0.0130 \\
\hline Abell 754 & 21.02 & 0 & 20.68 & 0 & 13.85 & 0.0170 & 13.58 & 0.0180 & 13.66 & 0.0300 \\
\hline Abell 773 & 21.38 & 0 & 20.91 & 0 & 15.46 & 0.0850 & 15.32 & 0.0970 & 15.13 & 0.0890 \\
\hline
\end{tabular}


Table 4-Continued

\begin{tabular}{|c|c|c|c|c|c|c|c|c|c|c|}
\hline $\begin{array}{c}\text { Name } \\
\text { Cluster Name }\end{array}$ & $\begin{array}{l}\text { Flux } \\
\text { NUV } \\
\text { (mag) }\end{array}$ & $\begin{array}{l}\text { Error } \\
\text { NUV } \\
\text { (mag) }\end{array}$ & $\begin{array}{l}\text { Flux } \\
\text { FUV } \\
\text { (mag) }\end{array}$ & $\begin{array}{l}\text { Error } \\
\text { FUV } \\
\text { (mag) }\end{array}$ & $\begin{array}{c}\text { Flux } \\
\text { J } \\
(\mathrm{mag})\end{array}$ & $\begin{array}{c}\text { Error } \\
\quad J \\
(\mathrm{mag})\end{array}$ & $\begin{array}{c}\text { Flux } \\
\text { H } \\
(\mathrm{mag})\end{array}$ & $\begin{array}{c}\text { Error } \\
\text { H } \\
(\mathrm{mag})\end{array}$ & $\begin{array}{c}\text { Flux } \\
\text { K } \\
(\mathrm{mag})\end{array}$ & $\begin{array}{c}\text { Error } \\
\mathrm{K} \\
(\mathrm{mag})\end{array}$ \\
\hline Abell 907 & 21.19 & 0 & 20.83 & 0 & 15.19 & 0.0560 & 14.87 & 0.0600 & 14.67 & 0.0720 \\
\hline Abell 963 & 21.62 & 0.0411 & 21.97 & 0.0409 & 14.59 & 0.0320 & 14.35 & 0.0360 & 14.25 & 0.0309 \\
\hline Abell 1060 & 17.74 & 0.0507 & 18.49 & 0.1080 & 11.33 & 0.0200 & 11.32 & 0.0200 & 11.52 & 0.0300 \\
\hline Abell 1063S & 21.67 & 0 & 21.20 & 0 & 15.89 & 0.1430 & 15.33 & 0.1240 & 15.52 & 0.1680 \\
\hline Abell 1068 & 19.39 & 0.0322 & $\ldots$ & $\ldots$ & 14.04 & 0.0170 & 13.86 & 0.0220 & 13.73 & 0.0210 \\
\hline Abell 1201 & 21.51 & 0 & 21.05 & 0 & 15.17 & 0.0480 & 14.99 & 0.0690 & 14.75 & 0.0590 \\
\hline Abell $1204^{\mathrm{a}}$ & 21.33 & 0.0605 & 21.24 & 0.0694 & 15.35 & 0.0340 & 15.31 & 0.0520 & 15.19 & 0.0505 \\
\hline Abell 1240 & $\ldots$ & $\ldots$ & $\ldots$ & $\ldots$ & $\ldots$ & $\ldots$ & $\ldots$ & $\ldots$ & $\ldots$ & $\ldots$ \\
\hline Abell 1361 & 21.32 & 0 & 20.87 & 0 & 14.84 & 0.0380 & 14.64 & 0.0540 & 14.56 & 0.0550 \\
\hline Abell 1413 & 20.67 & 0.0858 & 21.10 & 0.1193 & 13.77 & 0.0130 & 13.52 & 0.0160 & 13.54 & 0.0183 \\
\hline Abell 1423 & 21.43 & 0 & 20.97 & 0 & 15.64 & 0.0760 & 15.27 & 0.0770 & 15.09 & 0.0790 \\
\hline Abell 1446 & 21.33 & 0.3012 & 20.89 & 0 & 14.10 & 0.0150 & 13.99 & 0.0220 & 14.00 & 0.0213 \\
\hline Abell 1569 & 20.94 & 0.2780 & 20.89 & 0 & 14.10 & 0.0110 & 13.86 & 0.0150 & 14.03 & 0.0157 \\
\hline Abell 1576 & $\ldots$ & $\ldots$ & $\ldots$ & $\ldots$ & $\ldots$ & $\ldots$ & $\ldots$ & $\ldots$ & $\ldots$ & $\ldots$ \\
\hline Abell 1644 & 18.90 & 0.0421 & 19.67 & 0.2327 & 12.38 & 0.0060 & 12.19 & 0.0060 & 12.41 & 0.0087 \\
\hline Abell 1650 & 21.45 & 0 & 20.99 & 0 & 14.34 & 0.0290 & 14.07 & 0.0350 & 14.23 & 0.0540 \\
\hline Abell 1651 & $\ldots$ & $\ldots$ & $\ldots$ & $\ldots$ & $\ldots$ & $\ldots$ & $\ldots$ & $\ldots$ & $\ldots$ & $\ldots$ \\
\hline Abell 1664 & 17.77 & 0.0194 & 18.16 & 0.0336 & 14.28 & 0.0290 & 14.11 & 0.0350 & 14.42 & 0.0378 \\
\hline Abell 1689 & 21.28 & 0 & 20.84 & 0 & 15.30 & 0.0660 & 15.16 & 0.0860 & 14.88 & 0.0920 \\
\hline Abell 1736 & 19.51 & 0.1769 & 20.59 & 0 & 12.77 & 0.0050 & 12.57 & 0.0060 & 12.77 & 0.0089 \\
\hline Abell 1758 & $\ldots$ & $\ldots$ & $\ldots$ & $\ldots$ & $\ldots$ & $\ldots$ & $\ldots$ & $\ldots$ & $\ldots$ & $\ldots$ \\
\hline Abell 1763 & 22.27 & 0.07 & 22.56 & 0.13 & 16.07 & 0.0870 & 16.08 & 0.1100 & 15.06 & 0.0709 \\
\hline Abell 1795 & 18.00 & 0.0287 & 18.48 & 0.1079 & 13.25 & 0.0070 & 12.96 & 0.0080 & 13.12 & 0.0105 \\
\hline Abell 1835 & 18.92 & 0.0179 & 19.15 & 0.0297 & 14.93 & 0.0380 & 14.62 & 0.0430 & 14.58 & 0.0468 \\
\hline Abell 1914 & 22.46 & 0.1264 & 22.98 & 0.1598 & 15.56 & 0.0400 & 15.35 & 0.0460 & 15.49 & 0.0682 \\
\hline Abell 1942 & 23.27 & 0 & 23.19 & 0.3217 & 15.47 & 0.0900 & 15.44 & 0.1180 & 14.94 & 0.0960 \\
\hline Abell 1991 & 19.71 & 0.0619 & 21.14 & 0 & 13.05 & 0.0080 & 12.82 & 0.0080 & 13.04 & 0.0138 \\
\hline Abell 1995 & 23.07 & 0.2903 & 23.30 & 0.3020 & 16.15 & 0.0660 & 15.77 & 0.0710 & 15.50 & 0.0569 \\
\hline Abell 2029a & 19.14 & 0.0473 & 19.99 & 0.0797 & 12.56 & 0.0060 & 12.32 & 0.0060 & 12.42 & 0.0096 \\
\hline Abell 2034 & 21.65 & 0 & 21.18 & 0 & 15.04 & 0.0460 & 14.81 & 0.0570 & 14.78 & 0.0630 \\
\hline Abell $2052^{\mathrm{a}}$ & 19.12 & 0.1005 & 19.47 & 0.1726 & 12.22 & 0.0050 & 12.02 & 0.0050 & 12.23 & 0.0074 \\
\hline Abell 2063 & 19.06 & 0.0298 & 20.77 & 0 & 12.48 & 0.0050 & 12.29 & 0.0060 & 12.53 & 0.0094 \\
\hline Abell 2065 & 21.19 & 0.3182 & 20.96 & 0 & 14.21 & 0.0333 & 13.99 & 0.0474 & 14.04 & 0.0406 \\
\hline Abell 2069 & 21.54 & 0 & 21.09 & 0 & 14.79 & 0.0390 & 14.46 & 0.0480 & 14.55 & 0.0510 \\
\hline Abell 2104 & 19.02 & 0 & 19.08 & 0 & 15.53 & 0.0880 & 15.12 & 0.0830 & 15.06 & 0.1090 \\
\hline Abell 2107 & 19.26 & 0.1134 & 20.05 & 0.2199 & 12.21 & 0.0040 & 11.99 & 0.0040 & 12.21 & 0.0065 \\
\hline Abell 2111 & 22.61 & 0.0996 & 23.69 & 0.2615 & 15.38 & 0.0560 & 15.26 & 0.0790 & 14.78 & 0.0601 \\
\hline Abell 2124 & 20.08 & 0.1435 & 20.17 & 0.1617 & 13.05 & 0.0070 & 12.88 & 0.0090 & 12.96 & 0.0091 \\
\hline Abell 2125 & 21.38 & 0 & 20.94 & 0 & 15.50 & 0.0860 & 15.20 & 0.0930 & 15.10 & 0.1070 \\
\hline Abell $2142^{\mathrm{a}}$ & 20.48 & 0.0320 & 21.44 & 0.0714 & 13.73 & 0.0150 & 13.56 & 0.0170 & 13.65 & 0.0250 \\
\hline Abell 2147 & 19.20 & 0.0797 & 20.36 & 0.2335 & 12.44 & 0.0040 & 12.25 & 0.0050 & 12.43 & 0.0068 \\
\hline Abell 2151 & 19.43 & 0.1109 & 21.00 & 0 & 12.37 & 0.0040 & 12.20 & 0.0050 & 12.39 & 0.0058 \\
\hline Abell 2163 & $\ldots$ & $\ldots$ & $\ldots$ & $\ldots$ & $\ldots$ & $\ldots$ & $\ldots$ & $\ldots$ & $\ldots$ & $\ldots$ \\
\hline Abell 2187 & 21.06 & 0.1970 & 21.26 & 0.4139 & 14.69 & 0.0250 & 14.49 & 0.0280 & 14.38 & 0.0326 \\
\hline
\end{tabular}


Table 4-Continued

\begin{tabular}{|c|c|c|c|c|c|c|c|c|c|c|}
\hline $\begin{array}{c}\text { Name } \\
\text { Cluster Name }\end{array}$ & $\begin{array}{l}\text { Flux } \\
\text { NUV } \\
\text { (mag) }\end{array}$ & $\begin{array}{l}\text { Error } \\
\text { NUV } \\
\text { (mag) }\end{array}$ & $\begin{array}{l}\text { Flux } \\
\text { FUV } \\
\text { (mag) }\end{array}$ & $\begin{array}{l}\text { Error } \\
\text { FUV } \\
\text { (mag) }\end{array}$ & $\begin{array}{c}\text { Flux } \\
\text { J } \\
(\mathrm{mag})\end{array}$ & $\begin{array}{c}\text { Error } \\
\mathrm{J} \\
\text { (mag) }\end{array}$ & $\begin{array}{c}\text { Flux } \\
\text { H } \\
(\mathrm{mag})\end{array}$ & $\begin{array}{c}\text { Error } \\
\text { H } \\
(\mathrm{mag})\end{array}$ & $\begin{array}{c}\text { Flux } \\
\text { K } \\
\text { (mag) }\end{array}$ & $\begin{array}{c}\text { Error } \\
\mathrm{K} \\
(\mathrm{mag})\end{array}$ \\
\hline Abell 2199 & 18.42 & 0.0685 & 19.15 & 0.0516 & 11.68 & 0.0030 & 11.45 & 0.0040 & 11.67 & 0.0045 \\
\hline Abell 2204 & $\ldots$ & $\ldots$ & $\ldots$ & $\ldots$ & $\ldots$ & $\ldots$ & $\ldots$ & $\ldots$ & $\ldots$ & $\ldots$ \\
\hline Abell 2218 & 22.27 & 0.0709 & 23.58 & 0.3181 & $\ldots$ & $\ldots$ & $\ldots$ & $\ldots$ & $\ldots$ & $\ldots$ \\
\hline Abell 2219 & 23.03 & 0 & 23.02 & 0 & 15.83 & 0.1060 & 15.24 & 0.0970 & 15.38 & 0.1270 \\
\hline Abell 2244 & $\ldots$ & $\ldots$ & $\ldots$ & $\ldots$ & $\ldots$ & $\ldots$ & $\ldots$ & $\ldots$ & $\ldots$ & $\ldots$ \\
\hline Abell 2255 & 20.61 & 0.0353 & 21.54 & 0.0876 & 14.76 & 0.0270 & 14.49 & 0.0360 & 14.53 & 0.0396 \\
\hline Abell 2256 & 20.05 & 0.1159 & 20.60 & 0.2400 & 13.06 & 0.0210 & 12.82 & 0.0240 & 13.01 & 0.0290 \\
\hline Abell 2259 & 21.37 & 0 & 20.95 & 0 & 15.04 & 0.0490 & 14.78 & 0.0530 & 14.72 & 0.0610 \\
\hline Abell 2261 & $\ldots$ & $\ldots$ & $\ldots$ & $\ldots$ & $\ldots$ & $\ldots$ & $\ldots$ & $\ldots$ & $\ldots$ & $\ldots$ \\
\hline Abell 2294 & $\ldots$ & $\ldots$ & $\ldots$ & $\ldots$ & $\ldots$ & $\ldots$ & $\ldots$ & $\ldots$ & $\ldots$ & $\ldots$ \\
\hline Abell 2319 & 22.47 & 0 & 20.58 & 0.1400 & 13.85 & 0.0180 & 13.56 & 0.0200 & 13.68 & 0.0240 \\
\hline Abell 2384 & $\ldots$ & $\ldots$ & $\ldots$ & $\ldots$ & $\ldots$ & $\ldots$ & $\ldots$ & $\ldots$ & $\ldots$ & $\ldots$ \\
\hline Abell 2390 & 19.63 & 0.0462 & 19.70 & 0.0443 & 15.17 & 0.0540 & 15.23 & 0.0690 & 14.59 & 0.0508 \\
\hline Abell 2409 & 20.71 & 0 & 20.46 & 0 & 15.23 & 0.0730 & 15.10 & 0.0950 & 14.83 & 0.0910 \\
\hline Abell 2420 & $\ldots$ & $\ldots$ & $\ldots$ & $\ldots$ & $\ldots$ & $\ldots$ & $\ldots$ & $\ldots$ & $\ldots$ & $\ldots$ \\
\hline Abell 2462 & 20.30 & 0.1821 & 21.30 & 0.3477 & 13.47 & 0.0100 & 13.28 & 0.0140 & 13.41 & 0.0147 \\
\hline Abell 2537 & $\ldots$ & $\ldots$ & $\ldots$ & $\ldots$ & $\ldots$ & $\ldots$ & $\ldots$ & $\ldots$ & $\ldots$ & $\ldots$ \\
\hline Abell 2554 & 21.12 & 0.2837 & 21.17 & 0 & 14.14 & 0.0170 & 13.90 & 0.0270 & 14.07 & 0.0273 \\
\hline Abell 2556 & 20.68 & 0.2537 & 21.14 & 0.3075 & 13.71 & 0.0150 & 13.60 & 0.0230 & 13.67 & 0.0224 \\
\hline Abell 2589 & 19.39 & 0.0912 & 20.00 & 0.0780 & 12.46 & 0.0050 & 12.23 & 0.0050 & 12.45 & 0.0084 \\
\hline Abell $2597^{\mathrm{a}}$ & 18.68 & 0.0210 & 18.95 & 0.0303 & 13.91 & 0.0200 & 13.92 & 0.0320 & 13.90 & 0.0301 \\
\hline Abell 2626 & 19.17 & 0.1195 & 20.04 & 0.2993 & 12.77 & 0.0070 & 12.56 & 0.0090 & 12.78 & 0.0113 \\
\hline Abell 2631 & 22.48 & 0 & 23.03 & 0 & 16.21 & 0.1430 & 15.81 & 0.1490 & 15.68 & 0.1470 \\
\hline Abell 2657 & 20.76 & 0 & 20.55 & 0 & 14.07 & 0.0250 & 13.83 & 0.0310 & 13.96 & 0.0380 \\
\hline Abell 2667 & 20.64 & 0.1827 & 19.83 & 0.1478 & 15.11 & 0.0490 & 15.24 & 0.0920 & 14.85 & 0.0666 \\
\hline Abell 2717 & 20.01 & 0.2005 & 20.94 & 0 & 13.03 & 0.0070 & 12.79 & 0.0080 & 12.89 & 0.0121 \\
\hline Abell 2744a & $\ldots$ & $\ldots$ & $\ldots$ & $\ldots$ & $\ldots$ & $\ldots$ & $\ldots$ & $\ldots$ & $\ldots$ & $\ldots$ \\
\hline Abell 2744b & $\ldots$ & $\ldots$ & $\ldots$ & $\ldots$ & $\ldots$ & $\ldots$ & $\ldots$ & $\ldots$ & $\ldots$ & $\ldots$ \\
\hline Abell 2813 & 21.35 & 0 & 20.90 & 0 & 16.82 & 0.2460 & 16.70 & 0.3290 & 15.84 & 0.1620 \\
\hline Abell 3084 & 20.94 & 0.09 & 22 & 0.3 & 14.61 & 0.0310 & 14.09 & 0.0320 & 14.05 & 0.0324 \\
\hline Abell 3088 & $\ldots$ & $\ldots$ & $\ldots$ & $\ldots$ & $\ldots$ & $\ldots$ & $\ldots$ & $\ldots$ & $\ldots$ & $\ldots$ \\
\hline Abell $3112^{\mathrm{a}}$ & 19.81 & 0.0371 & 20.18 & 0.0596 & 13.17 & 0.0080 & 13.00 & 0.0090 & 12.99 & 0.0109 \\
\hline Abell 3120 & 20.10 & 0.0925 & 20.93 & 0.1769 & 13.52 & 0.0120 & 13.30 & 0.0130 & 13.41 & 0.0166 \\
\hline Abell 3158 & 20.02 & 0.1791 & 19.78 & 0.1559 & 13.00 & 0.0080 & 12.69 & 0.0100 & 12.92 & 0.0117 \\
\hline Abell 3266 & 19.40 & 0.0336 & 20.37 & 0.0786 & 12.54 & 0.0050 & 12.29 & 0.0060 & 12.43 & 0.0084 \\
\hline Abell 3364 & $\ldots$ & $\ldots$ & $\ldots$ & $\ldots$ & $\ldots$ & $\ldots$ & $\ldots$ & $\ldots$ & $\ldots$ & $\ldots$ \\
\hline Abell 3376 & 19.55 & 0.0510 & 20.27 & 0.0882 & 13.20 & 0.0110 & 12.98 & 0.0120 & 13.17 & 0.0162 \\
\hline Abell 3391 & $\ldots$ & $\ldots$ & $\ldots$ & $\ldots$ & $\ldots$ & $\ldots$ & $\ldots$ & $\ldots$ & $\ldots$ & $\ldots$ \\
\hline Abell 3395 & $\ldots$ & $\ldots$ & $\ldots$ & $\ldots$ & $\ldots$ & $\ldots$ & $\ldots$ & $\ldots$ & $\ldots$ & $\ldots$ \\
\hline Abell 3528S & 18.58 & 0.0317 & 19.84 & 0.0860 & 11.91 & 0.0040 & 11.70 & 0.0040 & 11.96 & 0.0059 \\
\hline Abell 3558 & 18.99 & 0.0625 & 19.70 & 0.1114 & 12.16 & 0.0040 & 11.94 & 0.0040 & 12.16 & 0.0069 \\
\hline Abell 3562 & 19.63 & 0.1968 & 20.60 & 0 & 12.90 & 0.0070 & 12.70 & 0.0070 & 12.90 & 0.0102 \\
\hline Abell 3571 & $\ldots$ & $\ldots$ & $\ldots$ & $\ldots$ & $\ldots$ & $\ldots$ & $\ldots$ & $\ldots$ & $\ldots$ & $\ldots$ \\
\hline Abell 3581 & 18.06 & 0.0824 & 19.21 & 0.1843 & 11.82 & 0.0030 & 11.61 & 0.0030 & 11.87 & 0.0046 \\
\hline
\end{tabular}


Table 4-Continued

\begin{tabular}{|c|c|c|c|c|c|c|c|c|c|c|}
\hline $\begin{array}{c}\text { Name } \\
\text { Cluster Name }\end{array}$ & $\begin{array}{l}\text { Flux } \\
\text { NUV } \\
\text { (mag) }\end{array}$ & $\begin{array}{l}\text { Error } \\
\text { NUV } \\
\text { (mag) }\end{array}$ & $\begin{array}{l}\text { Flux } \\
\text { FUV } \\
\text { (mag) }\end{array}$ & $\begin{array}{l}\text { Error } \\
\text { FUV } \\
\text { (mag) }\end{array}$ & $\begin{array}{c}\text { Flux } \\
\text { J } \\
\text { (mag) }\end{array}$ & $\begin{array}{c}\text { Error } \\
\mathrm{J} \\
(\mathrm{mag})\end{array}$ & $\begin{array}{c}\text { Flux } \\
\text { H } \\
(\mathrm{mag})\end{array}$ & $\begin{array}{c}\text { Error } \\
\mathrm{H} \\
(\mathrm{mag})\end{array}$ & $\begin{array}{c}\text { Flux } \\
\text { K } \\
\text { (mag) }\end{array}$ & $\begin{array}{c}\text { Error } \\
\mathrm{K} \\
(\mathrm{mag})\end{array}$ \\
\hline Abell 3667 & $\ldots$ & $\ldots$ & $\ldots$ & $\ldots$ & $\ldots$ & $\ldots$ & $\ldots$ & $\ldots$ & $\ldots$ & $\ldots$ \\
\hline Abell 3822 & 20.28 & 0.1675 & 20.91 & 0.3265 & 13.39 & 0.0140 & 13.18 & 0.0150 & 13.32 & 0.0164 \\
\hline Abell 3827 & 18.99 & 0.0680 & 20.95 & 0.3039 & 13.08 & 0.0100 & 12.87 & 0.0120 & 13.01 & 0.0142 \\
\hline Abell 3921 & 20.31 & 0.0367 & 20.75 & 0.1031 & 13.44 & 0.0130 & 13.16 & 0.0150 & 13.15 & 0.0161 \\
\hline Abell 4038 & $\ldots$ & $\ldots$ & $\ldots$ & $\ldots$ & $\ldots$ & $\ldots$ & $\ldots$ & $\ldots$ & $\ldots$ & $\ldots$ \\
\hline Abell 4059 & 19.10 & 0.0407 & 21.22 & 0 & 12.45 & 0.0050 & 12.21 & 0.0050 & 12.38 & 0.0070 \\
\hline Abell S0405 & 19.44 & 0.1960 & 20.59 & 0 & 12.85 & 0.0090 & 12.63 & 0.0100 & 12.84 & 0.0132 \\
\hline Abell S0592 & $\ldots$ & $\ldots$ & $\ldots$ & $\ldots$ & $\ldots$ & $\ldots$ & $\ldots$ & $\ldots$ & $\ldots$ & $\ldots$ \\
\hline AC 114 & 21.70 & 0 & 21.24 & 0 & 16.08 & 0.1330 & 15.59 & 0.1250 & 15.65 & 0.1760 \\
\hline AWM7 & 18.28 & 0.1447 & 20.65 & 0 & 11.09 & 0.0020 & 10.91 & 0.0020 & 11.23 & 0.0022 \\
\hline CENTAURUS & 17.27 & 0.0734 & 18.53 & 0.2019 & 10.30 & 0.0100 & 10.40 & 0.0100 & 10.64 & 0.0200 \\
\hline CID 0072 & 19.15 & 0.0892 & 20.24 & 0.2343 & 12.63 & 0.0050 & 12.38 & 0.0060 & 12.61 & 0.0084 \\
\hline CL J1226.9+3332 & 24.17 & 0.1375 & 24.52 & 0 & $\ldots$ & $\ldots$ & $\ldots$ & $\ldots$ & $\ldots$ & $\ldots$ \\
\hline CYGNUS A & $\ldots$ & $\ldots$ & $\ldots$ & $\ldots$ & $\ldots$ & $\ldots$ & $\ldots$ & $\ldots$ & $\ldots$ & $\ldots$ \\
\hline ESO 3060170 & 19.06 & 0.0275 & $\ldots$ & $\ldots$ & 12.30 & 0.0050 & 12.10 & 0.0050 & 12.31 & 0.0071 \\
\hline ESO 5520200 & 18.84 & 0.1099 & 19.73 & 0.2356 & 11.92 & 0.0040 & 11.74 & 0.0040 & 12.01 & 0.0063 \\
\hline EXO 0422-086 & 18.59 & 0.1398 & 19.22 & 0 & 12.69 & 0.0040 & 12.53 & 0.0040 & 12.92 & 0.0077 \\
\hline HCG 0062 & 20.93 & 0 & 19.22 & 0.0436 & 12.11 & 0.0040 & 11.86 & 0.0050 & 12.04 & 0.0080 \\
\hline HCG 42 & 17.41 & 0.0331 & 18.24 & 0.0879 & 10.64 & 0.0010 & 10.43 & 0.0010 & 10.67 & 0.0017 \\
\hline HERCULES A ${ }^{a}$ & 20.90 & 0.1230 & 21.26 & 0.1296 & 14.50 & 0.0370 & 14.40 & 0.0450 & 14.33 & 0.0393 \\
\hline HYDRA A ${ }^{a}$ & 17.63 & 0.0499 & 18.20 & 0.0253 & 12.88 & 0.0100 & 12.67 & 0.0120 & 12.84 & 0.0131 \\
\hline M49 & 15.94 & 0.0208 & 16.77 & 0.0551 & 9.11 & 0.0100 & 9.06 & 0.0100 & 9.20 & 0.0100 \\
\hline M87 & 15.47 & 0.0012 & 15.74 & 0.0150 & 9.13 & 0.0100 & 9.00 & 0.0100 & 9.24 & 0.0100 \\
\hline MACS J0011.7-1523 & $\ldots$ & $\ldots$ & $\ldots$ & $\ldots$ & $\ldots$ & $\ldots$ & $\ldots$ & $\ldots$ & $\ldots$ & $\ldots$ \\
\hline MACS J0035.4-2015 & $\cdots$ & $\cdots$ & $\cdots$ & $\cdots$ & $\cdots$ & $\cdots$ & $\cdots$ & $\cdots$ & $\cdots$ & $\cdots$ \\
\hline MACS J0159.8-0849 & $\ldots$ & $\ldots$ & $\ldots$ & $\ldots$ & $\ldots$ & $\ldots$ & $\ldots$ & $\ldots$ & $\ldots$ & $\ldots$ \\
\hline MACS J0242.5-2132 & $\ldots$ & $\ldots$ & $\ldots$ & $\ldots$ & $\ldots$ & $\ldots$ & $\ldots$ & $\ldots$ & $\ldots$ & $\ldots$ \\
\hline MACS J0257.1-2325 & $\ldots$ & $\ldots$ & $\ldots$ & $\ldots$ & $\ldots$ & $\ldots$ & $\ldots$ & $\ldots$ & $\ldots$ & $\ldots$ \\
\hline MACS J0257.6-2209 & 21.82 & 0 & 21.35 & 0 & 15.95 & 0.1270 & 15.63 & 0.1610 & 15.33 & 0.1250 \\
\hline MACS J0308.9+2645 & 19.46 & 0 & 19.44 & 0 & 15.81 & 0.0850 & 15.58 & 0.1120 & 15.23 & 0.0960 \\
\hline MACS J0329.6-0211 & $\ldots$ & $\ldots$ & $\ldots$ & $\ldots$ & $\ldots$ & $\ldots$ & $\ldots$ & $\ldots$ & $\ldots$ & $\ldots$ \\
\hline MACS J0417.5-1154 & $\ldots$ & $\ldots$ & $\ldots$ & $\ldots$ & $\ldots$ & $\ldots$ & $\ldots$ & $\ldots$ & $\ldots$ & $\ldots$ \\
\hline MACS J0429.6-0253 & $\ldots$ & $\ldots$ & $\ldots$ & $\ldots$ & $\ldots$ & $\ldots$ & $\ldots$ & $\ldots$ & $\ldots$ & $\ldots$ \\
\hline MACS J0520.7-1328 & $\ldots$ & $\cdots$ & $\ldots$ & $\ldots$ & $\ldots$ & $\ldots$ & $\ldots$ & $\ldots$ & $\ldots$ & $\ldots$ \\
\hline MACS J0547.0-3904 & $\ldots$ & $\ldots$ & $\ldots$ & $\ldots$ & $\ldots$ & $\ldots$ & $\ldots$ & $\ldots$ & $\ldots$ & $\ldots$ \\
\hline MACS J0717.5+3745 & $\ldots$ & $\ldots$ & $\ldots$ & $\ldots$ & $\ldots$ & $\ldots$ & $\ldots$ & $\cdots$ & $\ldots$ & $\ldots$ \\
\hline MACS J0744.8+3927 & $\ldots$ & $\ldots$ & $\ldots$ & $\ldots$ & $\ldots$ & $\ldots$ & $\ldots$ & $\ldots$ & $\ldots$ & $\ldots$ \\
\hline MACS J1115.2+5320 & $\ldots$ & $\ldots$ & $\ldots$ & $\ldots$ & $\ldots$ & $\ldots$ & $\ldots$ & $\ldots$ & $\ldots$ & $\ldots$ \\
\hline MACS J115.8+0129 & $\ldots$ & $\ldots$ & $\ldots$ & $\ldots$ & $\ldots$ & $\ldots$ & $\ldots$ & $\ldots$ & $\ldots$ & $\ldots$ \\
\hline MACS J1131.8-1955 & $\ldots$ & $\ldots$ & $\ldots$ & $\ldots$ & $\ldots$ & $\ldots$ & $\ldots$ & $\ldots$ & $\ldots$ & $\ldots$ \\
\hline MACS J1149.5+2223 & $\ldots$ & $\ldots$ & $\ldots$ & $\ldots$ & $\ldots$ & $\ldots$ & $\ldots$ & $\ldots$ & $\ldots$ & $\ldots$ \\
\hline MACS J1206.2-0847 & $\ldots$ & $\ldots$ & $\ldots$ & $\ldots$ & $\ldots$ & $\ldots$ & $\ldots$ & $\cdots$ & $\ldots$ & $\ldots$ \\
\hline MACS J1311.0-0310 & $\ldots$ & $\ldots$ & $\ldots$ & $\ldots$ & $\ldots$ & $\ldots$ & $\ldots$ & $\ldots$ & $\ldots$ & $\ldots$ \\
\hline MACS J1621.3+3810 & $\ldots$ & $\ldots$ & $\ldots$ & $\ldots$ & $\ldots$ & $\ldots$ & $\ldots$ & $\ldots$ & $\ldots$ & $\ldots$ \\
\hline
\end{tabular}


Table 4-Continued

\begin{tabular}{|c|c|c|c|c|c|c|c|c|c|c|}
\hline $\begin{array}{c}\text { Name } \\
\text { Cluster Name }\end{array}$ & $\begin{array}{l}\text { Flux } \\
\text { NUV } \\
\text { (mag) }\end{array}$ & $\begin{array}{l}\text { Error } \\
\text { NUV } \\
\text { (mag) }\end{array}$ & $\begin{array}{l}\text { Flux } \\
\text { FUV } \\
\text { (mag) }\end{array}$ & $\begin{array}{l}\text { Error } \\
\text { FUV } \\
\text { (mag) }\end{array}$ & $\begin{array}{c}\text { Flux } \\
\text { J } \\
\text { (mag) }\end{array}$ & $\begin{array}{c}\text { Error } \\
\mathrm{J} \\
(\mathrm{mag})\end{array}$ & $\begin{array}{c}\text { Flux } \\
\text { H } \\
(\mathrm{mag})\end{array}$ & $\begin{array}{c}\text { Error } \\
\text { H } \\
(\mathrm{mag})\end{array}$ & $\begin{array}{c}\text { Flux } \\
\text { K } \\
(\mathrm{mag})\end{array}$ & $\begin{array}{c}\text { Error } \\
\mathrm{K} \\
(\mathrm{mag})\end{array}$ \\
\hline MACS J1931.8-2634 & $\cdots$ & $\cdots$ & $\cdots$ & $\cdots$ & $\cdots$ & $\cdots$ & $\cdots$ & $\cdots$ & $\cdots$ & $\cdots$ \\
\hline MACS J2049.9-3217 & $\cdots$ & $\cdots$ & $\cdots$ & $\cdots$ & $\cdots$ & $\cdots$ & $\cdots$ & $\cdots$ & $\cdots$ & $\cdots$ \\
\hline MACS J2211.7-0349 & $\cdots$ & $\cdots$ & $\cdots$ & $\cdots$ & $\cdots$ & $\cdots$ & $\cdots$ & $\cdots$ & $\cdots$ & $\cdots$ \\
\hline MACS J2214.9-1359 & $\ldots$ & $\ldots$ & $\ldots$ & $\cdots$ & $\ldots$ & $\ldots$ & $\ldots$ & $\ldots$ & $\ldots$ & .. \\
\hline MACS J2228+2036 & $\ldots$ & $\ldots$ & $\ldots$ & $\ldots$ & $\ldots$ & $\cdots$ & $\cdots$ & $\cdots$ & $\cdots$ & $\cdots$ \\
\hline MACS J2229.7-2755 & 20.48 & 0.0733 & 20.24 & 0.0631 & 15.88 & 0.0720 & 15.42 & 0.0800 & 14.94 & 0.0616 \\
\hline MACS J2245.0+2637 & 21.19 & 0 & 20.83 & 0 & 15.99 & 0.1200 & 16.00 & 0.1860 & 15.52 & 0.1410 \\
\hline 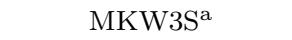 & 19.59 & 0.0366 & 20.50 & 0.0754 & 12.88 & 0.0060 & 12.66 & 0.0070 & 12.90 & 0.0112 \\
\hline MKW $04^{\mathrm{a}}$ & 18.03 & 0.0132 & 18.59 & 0.0281 & 11.19 & 0.0020 & 10.97 & 0.0020 & 11.20 & 0.0029 \\
\hline MKW 08 & 18.61 & 0.0285 & 19.50 & 0.0785 & 12.53 & 0.0070 & 12.35 & 0.0080 & 12.50 & 0.0107 \\
\hline MS 0016.9+1609 & $\ldots$ & $\ldots$ & $\ldots$ & $\ldots$ & $\ldots$ & $\ldots$ & $\ldots$ & $\ldots$ & $\ldots$ & $\ldots$ \\
\hline MS 0116.3-0115 & 19.39 & 0.0267 & 20.18 & 0.0363 & 12.59 & 0.0050 & 12.39 & 0.0060 & 12.62 & 0.0078 \\
\hline MS 0440.5+0204 & 20.22 & 0 & 20.32 & 0 & 14.38 & 0.0440 & 14.13 & 0.0470 & 13.99 & 0.0470 \\
\hline MS 0451.6-0305 & $\cdots$ & $\cdots$ & $\cdots$ & $\ldots$ & $\ldots$ & $\cdots$ & $\ldots$ & $\ldots$ & $\cdots$ & $\cdots$ \\
\hline MS 0735.6+7421 & 21.84 & 0.2980 & 21.14 & 0.2261 & 15.42 & 0.0500 & 15.15 & 0.0680 & 15.02 & 0.0625 \\
\hline MS $0839.8+2938^{\mathrm{a}}$ & 20.89 & 0.2499 & 20.67 & 0.2304 & 15.13 & 0.0280 & 14.93 & 0.0320 & 15.00 & 0.0386 \\
\hline MS 0906.5+1110 & $\ldots$ & $\ldots$ & $\ldots$ & $\ldots$ & $\ldots$ & $\ldots$ & $\ldots$ & $\ldots$ & $\ldots$ & $\ldots$ \\
\hline MS $1006.0+1202$ & $\cdots$ & $\cdots$ & $\cdots$ & $\cdots$ & $\cdots$ & $\cdots$ & $\cdots$ & $\cdots$ & $\cdots$ & $\cdots$ \\
\hline MS 1008.1-1224 & $\ldots$ & $\ldots$ & $\ldots$ & $\ldots$ & $\ldots$ & $\ldots$ & $\ldots$ & $\ldots$ & $\ldots$ & $\cdots$ \\
\hline MS $1455.0+2232^{\mathrm{a}}$ & 20.22 & 0.0337 & 20.09 & 0.0460 & 15.44 & 0.1020 & 15.33 & 0.1398 & 14.92 & 0.1033 \\
\hline MS 2137.3-2353 & 21.54 & 0.0482 & 21.45 & 0.0294 & 15.89 & 0.0640 & 15.65 & 0.0790 & 15.16 & 0.0643 \\
\hline MS J1157.3+5531 & 20.96 & 0.1020 & 21.30 & 0 & 13.67 & 0.0178 & 13.40 & 0.0218 & 13.54 & 0.0248 \\
\hline NGC 507 & 17.91 & 0.0178 & 19.08 & 0.0486 & -0.02 & 0.0010 & 10.79 & 0.0020 & 11.05 & 0.0018 \\
\hline NGC 4636 & 16.65 & 0.0285 & 17.46 & 0.0685 & 9.93 & 0.0200 & 9.88 & 0.0200 & 10.05 & 0.0300 \\
\hline NGC 5044 & 17.13 & 0.0121 & 17.94 & 0.0284 & 10.44 & 0.0010 & 10.26 & 0.0010 & 10.54 & 0.0019 \\
\hline NGC 5813 & 17.23 & 0.0099 & 18.42 & 0.0249 & 10.26 & 0.0300 & 10.29 & 0.0300 & 10.50 & 0.0300 \\
\hline NGC 5846 & 16.80 & 0.0101 & 17.51 & 0.0270 & 9.98 & 0.0010 & 9.78 & 0.0010 & 10.06 & 0.0011 \\
\hline OPHIUCHUS & 16.24 & 0.2065 & 17.80 & 0 & 11.04 & 0.0080 & 11.22 & 0.0070 & 12.06 & 0.0072 \\
\hline PKS 0745-191 & $\cdots$ & $\cdots$ & $\cdots$ & $\cdots$ & $\cdots$ & $\cdots$ & $\cdots$ & $\cdots$ & $\cdots$ & $\cdots$ \\
\hline RBS 0461 & 18.19 & 0 & 18.46 & 0 & 13.32 & 0.0110 & 12.97 & 0.0110 & 13.01 & 0.0170 \\
\hline RBS 0533 & 17.28 & 0.0612 & 18.10 & 0.1333 & 10.85 & 0.0020 & 10.71 & 0.0020 & 11.07 & 0.0024 \\
\hline RBS 0797 & 21.30 & 0 & 20.40 & 0.2609 & $\ldots$ & $\cdots$ & $\ldots$ & $\ldots$ & $\ldots$ & $\ldots$ \\
\hline RCS J2327-0204 & 19.52 & 0.0512 & 20.08 & 0.0805 & $\ldots$ & $\ldots$ & $\ldots$ & $\ldots$ & $\cdots$ & $\cdots$ \\
\hline RXCJ0331.1-2100 & $\ldots$ & $\ldots$ & $\cdots$ & $\ldots$ & $\ldots$ & $\cdots$ & $\ldots$ & $\ldots$ & $\ldots$ & $\ldots$ \\
\hline RXC J1023.8-2715 & 20.74 & 0.3265 & 20.32 & 0.2099 & 15.23 & 0.0590 & 15.15 & 0.0680 & 15.11 & 0.0758 \\
\hline RX J0220.9-3829 & $\ldots$ & $\cdots$ & $\cdots$ & $\cdots$ & $\ldots$ & $\cdots$ & $\cdots$ & $\cdots$ & $\ldots$ & $\cdots$ \\
\hline RX J0232.2-4420 & $\cdots$ & $\cdots$ & $\cdots$ & $\cdots$ & $\ldots$ & $\cdots$ & $\cdots$ & $\cdots$ & $\cdots$ & $\ldots$ \\
\hline RX J0439+0520 & 19.93 & 0 & 19.88 & 0 & 15.48 & 0.0910 & 15.22 & 0.1110 & 14.89 & 0.1000 \\
\hline RX J0439.0+0715 & $\ldots$ & $\cdots$ & $\ldots$ & $\cdots$ & $\ldots$ & $\ldots$ & $\ldots$ & $\ldots$ & $\ldots$ & $\ldots$ \\
\hline RX J0528.9-3927 & $\ldots$ & $\ldots$ & $\ldots$ & $\ldots$ & $\ldots$ & $\ldots$ & $\ldots$ & $\ldots$ & $\ldots$ & .. \\
\hline RX J0647.7+7015 & $\ldots$ & $\cdots$ & $\ldots$ & $\cdots$ & $\ldots$ & $\ldots$ & $\ldots$ & $\ldots$ & $\cdots$ & $\ldots$ \\
\hline RX J0819.6+6336 & 21.13 & 0 & 20.73 & 0 & 14.32 & 0.0240 & 14.06 & 0.0280 & 14.06 & 0.0310 \\
\hline RX J1000.4+4409 & 22.27 & 0.1729 & 20.97 & 0 & 0.03 & $\ldots$ & $\ldots$ & $\ldots$ & $\ldots$ & $\ldots$ \\
\hline RX J1022.1+3830 & 20.11 & 0.2209 & 20.92 & 0 & 13.16 & 0.0080 & 12.94 & 0.0100 & 13.16 & 0.0110 \\
\hline
\end{tabular}


Table 4-Continued

\begin{tabular}{|c|c|c|c|c|c|c|c|c|c|c|}
\hline $\begin{array}{c}\text { Name } \\
\text { Cluster Name }\end{array}$ & $\begin{array}{l}\text { Flux } \\
\text { NUV } \\
\text { (mag) }\end{array}$ & $\begin{array}{l}\text { Error } \\
\text { NUV } \\
\text { (mag) }\end{array}$ & $\begin{array}{l}\text { Flux } \\
\text { FUV } \\
\text { (mag) }\end{array}$ & $\begin{array}{l}\text { Error } \\
\text { FUV } \\
\text { (mag) }\end{array}$ & $\begin{array}{c}\text { Flux } \\
\text { J } \\
(\mathrm{mag})\end{array}$ & $\begin{array}{c}\text { Error } \\
\mathrm{J} \\
(\mathrm{mag})\end{array}$ & $\begin{array}{c}\text { Flux } \\
\text { H } \\
\text { (mag) }\end{array}$ & $\begin{array}{c}\text { Error } \\
\text { H } \\
(\mathrm{mag})\end{array}$ & $\begin{array}{c}\text { Flux } \\
\text { K } \\
\text { (mag) }\end{array}$ & $\begin{array}{c}\text { Error } \\
\mathrm{K} \\
(\mathrm{mag})\end{array}$ \\
\hline RX J1130.0+3637 & 20.75 & 0.1684 & 20.84 & 0 & 14.35 & 0.0210 & 14.16 & 0.0280 & 14.19 & 0.0297 \\
\hline RX J1320.2+3308 & 21.40 & 0 & 20.88 & 0.2884 & 13.52 & 0.0110 & 13.27 & 0.0120 & 13.41 & 0.0170 \\
\hline RX J1347.5-1145 & 21.03 & 0.0534 & 21.39 & 0.0661 & $\ldots$ & $\ldots$ & $\ldots$ & $\ldots$ & $\ldots$ & $\ldots$ \\
\hline RX J1423.8+2404 & $\ldots$ & $\ldots$ & $\ldots$ & $\ldots$ & $\cdots$ & $\ldots$ & $\ldots$ & $\ldots$ & $\ldots$ & $\ldots$ \\
\hline RX J1504.1-0248 & 17.69 & 0.0118 & 17.53 & 0.0203 & 15.07 & 0.0510 & 14.78 & 0.0450 & 15.12 & 0.0692 \\
\hline RX J1532.9+3021 & $\ldots$ & $\ldots$ & $\ldots$ & $\ldots$ & $\ldots$ & $\ldots$ & $\ldots$ & $\ldots$ & $\ldots$ & $\ldots$ \\
\hline RX J1539.5-8335 & $\ldots$ & $\ldots$ & $\ldots$ & $\ldots$ & $\ldots$ & $\ldots$ & $\ldots$ & $\ldots$ & $\ldots$ & $\ldots$ \\
\hline RX J1720.1+2638 & 19.73 & 0.1018 & 20.72 & 0.2954 & 14.02 & 0.0210 & 13.85 & 0.0230 & 14.02 & 0.0288 \\
\hline RX J1720.2+3536 & $\ldots$ & $\ldots$ & $\ldots$ & $\ldots$ & $\ldots$ & $\ldots$ & $\ldots$ & $\ldots$ & $\ldots$ & $\ldots$ \\
\hline RX J1852.1+5711 & 21.34 & 0 & 20.95 & 0 & 15.21 & 0.0560 & 14.97 & 0.0630 & 14.95 & 0.0880 \\
\hline RX J2129.6+0005 & 21.37 & 0.0988 & 21.21 & 0.0766 & 14.86 & 0.0440 & 14.52 & 0.0490 & 14.87 & 0.0529 \\
\hline SC 1327-312 & 19.72 & 0.0616 & 20.72 & 0.1747 & 13.31 & 0.0090 & 13.14 & 0.0100 & 13.27 & 0.0162 \\
\hline SERSIC 159-03 & 19.06 & 0.0141 & 19.41 & 0.0229 & 13.12 & 0.0080 & 12.88 & 0.0090 & 13.09 & 0.0133 \\
\hline SS2B153 & 17.86 & 0.0451 & 18.51 & 0.0992 & 11.16 & 0.0020 & 10.96 & 0.0020 & 11.23 & 0.0026 \\
\hline UGC 03957 & 18.83 & 0.0630 & 19.89 & 0.1925 & 11.92 & 0.0030 & 11.72 & 0.0040 & 11.96 & 0.0041 \\
\hline UGC 12491 & $\ldots$ & $\ldots$ & $\ldots$ & $\ldots$ & $\ldots$ & $\ldots$ & $\ldots$ & $\ldots$ & $\ldots$ & $\ldots$ \\
\hline ZWCL 1215 & 21.28 & 0 & 20.82 & 0 & 14.27 & 0.0290 & 14.01 & 0.0320 & 14.02 & 0.0440 \\
\hline ZWCL $1358+6245^{\mathrm{a}}$ & 23.56 & 0.2803 & 23.16 & 0.1521 & $\ldots$ & $\ldots$ & $\ldots$ & $\ldots$ & $\ldots$ & $\ldots$ \\
\hline ZWCL 1742 & $\ldots$ & $\ldots$ & $\ldots$ & $\ldots$ & $\cdots$ & $\cdots$ & $\cdots$ & $\cdots$ & $\cdots$ & $\cdots$ \\
\hline ZWCL 1953 & $\ldots$ & $\ldots$ & $\ldots$ & $\ldots$ & $\ldots$ & $\ldots$ & $\ldots$ & $\ldots$ & $\ldots$ & $\ldots$ \\
\hline ZWCL $3146^{\mathrm{a}}$ & 19.38 & 0.0236 & 19.18 & 0.0249 & 15.87 & 0.0940 & 14.83 & 0.0620 & 14.97 & 0.0878 \\
\hline ZWICKY 2701 & 21.43 & 0 & 20.95 & 0 & 15.73 & 0.1110 & 15.62 & 0.1700 & 15.33 & 0.1210 \\
\hline $\mathrm{ZwCl} 0857.9+2107$ & 20.10 & 0.0320 & 19.77 & 0.0246 & 15.71 & 0.0760 & 15.85 & 0.0910 & 15.13 & 0.0681 \\
\hline
\end{tabular}

${ }^{a}$ BCGs are also in the Hicks et al. (2010) sample.

${ }^{\mathrm{b}} \mathrm{BCG}$ GALEX fluxes are taken from Hicks et al. (2010).

Note. - Fluxes reported with errors equal to 0 are $3 \sigma$ upper limits. For NUV upper limits, the 2MASS flux is matched with a $7^{\prime \prime}$ aperture such that it is similar in size to the GALEX PSF. 
Table 5. Spitzer Aperture Flux.

\begin{tabular}{|c|c|c|c|c|c|c|c|c|c|c|c|c|c|c|}
\hline $\begin{array}{c}\text { Name } \\
\text { Cluster Name }\end{array}$ & $\begin{array}{c}\text { Flux } \\
3.6 \mu m \\
\text { (mJy) }\end{array}$ & $\begin{array}{r}\text { Error } \\
3.6 \mu m \\
(\mathrm{mJy})\end{array}$ & $\begin{array}{c}\text { Flux } \\
4.5 \mu m \\
(\mathrm{mJy})\end{array}$ & $\begin{array}{c}\text { Error } \\
4.5 \mu m \\
(\mathrm{mJy})\end{array}$ & $\begin{array}{c}\text { Flux } \\
5.8 \mu m \\
(\mathrm{mJy})\end{array}$ & $\begin{array}{c}\text { Error } \\
5.8 \mu m \\
(\mathrm{mJy})\end{array}$ & $\begin{array}{c}\text { Flux } \\
8.0 \mu m \\
(\mathrm{mJy})\end{array}$ & $\begin{array}{c}\text { Error } \\
8.0 \mu m \\
(\mathrm{mJy})\end{array}$ & $\begin{array}{c}\text { Flux } \\
24 \mu m \\
\text { (mJy) }\end{array}$ & $\begin{array}{c}\text { Error } \\
24 \mu m \\
(\mathrm{mJy})\end{array}$ & $\begin{array}{c}\text { Flux } \\
70 \mu m \\
(\mathrm{mJy})\end{array}$ & $\begin{array}{l}\text { Error } \\
70 \mu m \\
(\mathrm{mJy})\end{array}$ & $\begin{array}{l}\text { Flux } \\
160 \mu m \\
(\mathrm{mJy})\end{array}$ & $\begin{array}{c}\text { Error } \\
160 \mu m \\
(\mathrm{mJy})\end{array}$ \\
\hline 1E0657 56a & 0.47 & 0.02 & 0.38 & 0.02 & 0.22 & 0.01 & 0.14 & 0.01 & 0.15 & 0.02 & 5.29 & 0 & $\ldots$ & $\ldots$ \\
\hline 1E0657 56b & 0.57 & 0.03 & 0.46 & 0.02 & 0.26 & 0.01 & 0.17 & 0.01 & 0.11 & 0.02 & $1.43^{\mathrm{a}}$ & 0 & $\ldots$ & $\ldots$ \\
\hline 2A $0335+096$ & 26.12 & 1.31 & 15.93 & 0.80 & 14.79 & 0.74 & 9.94 & 0.50 & $2.40^{\mathrm{b}}$ & 0.24 & 77.10 & 20.36 & $\ldots$ & $\ldots$ \\
\hline 2PIGG J0011.5-2850 & & $\ldots$ & $\ldots$ & & $\ldots$ & $\ldots$ & & $\ldots$ & $\ldots$ & $\ldots$ & $\ldots$ & $\ldots$ & $\ldots$ & $\ldots$ \\
\hline 2PIGG J2227.0-3041 & $\ldots$ & $\ldots$ & $\ldots$ & $\ldots$ & $\ldots$ & $\ldots$ & $\ldots$ & $\ldots$ & $\ldots$ & $\ldots$ & $\ldots$ & $\ldots$ & $\ldots$ & $\ldots$ \\
\hline 3C 28.0 & 0.88 & 0.04 & 0.67 & 0.03 & 0.35 & 0.02 & 0.43 & 0.02 & $0.55^{\mathrm{c}}$ & 0.08 & 6.78 & 0 & $\ldots$ & $\ldots$ \\
\hline 3C 295 & & $\ldots$ & $\ldots$ & $\ldots$ & $\ldots$ & $\ldots$ & $\ldots$ & $\ldots$ & $\ldots$ & $\ldots$ & $\ldots$ & $\ldots$ & $\ldots$ & $\ldots$ \\
\hline 3C 388 & 6.20 & 0.31 & 4.11 & 0.21 & 2.70 & 0.13 & 1.88 & 0.09 & 2.15 & 0.22 & $18.79^{\mathrm{d}}$ & 4.16 & 7.13 & 0 \\
\hline $4 \mathrm{C} 55.16$ & $\ldots$ & $\ldots$ & $\ldots$ & $\ldots$ & $\ldots$ & $\ldots$ & $\ldots$ & $\ldots$ & $\ldots$ & $\ldots$ & $\ldots$ & $\ldots$ & $\ldots$ & $\ldots$ \\
\hline Abell 13 & $\ldots$ & $\ldots$ & $\ldots$ & $\ldots$ & $\ldots$ & $\ldots$ & $\ldots$ & $\ldots$ & $\ldots$ & $\ldots$ & $\ldots$ & $\ldots$ & $\ldots$ & $\ldots$ \\
\hline Abell 68 & & $\ldots$ & $\ldots$ & $\ldots$ & $\ldots$ & $\ldots$ & $\ldots$ & $\ldots$ & 1.28 & 0.15 & $\ldots$ & $\ldots$ & $\ldots$ & $\ldots$ \\
\hline Abell 85 & 15.08 & 0.75 & 9.43 & 0.47 & 6.79 & 0.34 & 4.36 & 0.22 & $1.51^{\mathrm{b}} 0.16$ & $17.17^{\mathrm{d}}$ & 3.88 & $\ldots$ & $\ldots$ & \\
\hline Abell 119 & & $\ldots$ & $\ldots$ & & $\ldots$ & $\ldots$ & & $\ldots$ & $\ldots$ & $\ldots$ & & $\ldots$ & $\ldots$ & $\ldots$ \\
\hline Abell 133 & $\ldots$ & $\ldots$ & $\ldots$ & $\ldots$ & $\ldots$ & $\ldots$ & $\ldots$ & $\ldots$ & $\ldots$ & $\ldots$ & $\ldots$ & $\ldots$ & $\ldots$ & $\ldots$ \\
\hline Abell 141 & $\ldots$ & $\ldots$ & $\ldots$ & $\ldots$ & $\ldots$ & $\ldots$ & $\ldots$ & $\ldots$ & $\ldots$ & $\ldots$ & $\ldots$ & $\ldots$ & $\ldots$ & $\ldots$ \\
\hline Abell 160 & $\ldots$ & $\ldots$ & $\ldots$ & $\ldots$ & $\ldots$ & $\ldots$ & $\ldots$ & $\ldots$ & $\ldots$ & $\ldots$ & $\ldots$ & $\ldots$ & $\ldots$ & $\ldots$ \\
\hline Abell 193 & & $\ldots$ & $\ldots$ & & $\ldots$ & $\ldots$ & & $\ldots$ & $\ldots$ & $\ldots$ & $\ldots$ & $\ldots$ & $\ldots$ & $\ldots$ \\
\hline Abell 209 & 1.35 & 0.07 & 1.04 & 0.05 & 0.58 & 0.03 & 0.44 & 0.02 & 0.24 & 0.03 & $\ldots$ & $\ldots$ & $\ldots$ & $\ldots$ \\
\hline Abell 222 & $\ldots$ & $\ldots$ & $\ldots$ & $\ldots$ & $\ldots$ & $\ldots$ & $\ldots$ & $\ldots$ & $\ldots$ & $\ldots$ & $\ldots$ & $\ldots$ & $\ldots$ & $\ldots$ \\
\hline Abell 223 & $\ldots$ & $\ldots$ & $\ldots$ & $\ldots$ & $\ldots$ & $\ldots$ & $\ldots$ & $\ldots$ & $\ldots$ & $\ldots$ & $\ldots$ & $\ldots$ & $\ldots$ & $\ldots$ \\
\hline Abell 262 & 73.01 & 3.65 & 43.31 & 2.17 & 34.50 & 1.72 & 22.20 & 1.11 & 4.08 & 0.41 & 90.28 & 24.05 & $\ldots$ & $\ldots$ \\
\hline Abell 267 & 1.37 & 0.07 & 1.07 & 0.05 & 0.65 & 0.03 & 0.44 & 0.02 & 0.14 & 0.06 & $\ldots$ & $\ldots$ & $\ldots$ & $\ldots$ \\
\hline Abell 368 & $\ldots$ & $\ldots$ & $\ldots$ & $\ldots$ & $\ldots$ & $\ldots$ & $\ldots$ & $\ldots$ & 0.59 & 0.06 & $\ldots$ & $\ldots$ & $\ldots$ & $\ldots$ \\
\hline Abell 370 & 0.79 & 0.04 & 0.66 & 0.03 & 0.41 & 0.02 & 0.26 & 0.01 & $0.08^{\mathrm{b}}$ & 0.03 & 2.44 & 0 & $6.13^{\mathrm{a}}$ & 0 \\
\hline Abell 383 & 1.60 & 0.08 & 1.20 & 0.06 & 0.73 & 0.04 & 0.61 & 0.03 & $0.51^{\mathrm{b}}$ & 0.05 & $\ldots$ & $\ldots$ & $\ldots$ & $\ldots$ \\
\hline Abell 399 & & $\cdots$ & $\ldots$ & & $\cdots$ & $\ldots$ & & $\ldots$ & $\ldots$ & $\ldots$ & $\ldots$ & $\cdots$ & $\ldots$ & $\ldots$ \\
\hline Abell 400 & $\ldots$ & $\ldots$ & $\ldots$ & $\ldots$ & $\ldots$ & $\ldots$ & $\ldots$ & $\ldots$ & $\ldots$ & $\ldots$ & $\ldots$ & $\ldots$ & $\ldots$ & $\ldots$ \\
\hline Abell 401 & $\ldots$ & $\ldots$ & $\ldots$ & $\ldots$ & $\ldots$ & $\ldots$ & $\ldots$ & $\ldots$ & 0.49 & 0 & 5.33 & 0 & $\ldots$ & $\ldots$ \\
\hline Abell $426^{\mathrm{e}}$ & 143.49 & 7.17 & 124.20 & 6.21 & 208.70 & 10.44 & 356.70 & 17.84 & 2864.10 & 286.416 & 6312.40 & 1264.04 & 4115.45 & 5824.60 \\
\hline
\end{tabular}


Table 5-Continued

\begin{tabular}{|c|c|c|c|c|c|c|c|c|c|c|c|c|c|c|}
\hline $\begin{array}{c}\text { Name } \\
\text { Cluster Name }\end{array}$ & $\begin{array}{c}\text { Flux } \\
3.6 \mu m \\
(\mathrm{mJy})\end{array}$ & $\begin{array}{r}\text { Error } \\
3.6 \mu m \\
(\mathrm{mJy})\end{array}$ & $\begin{array}{l}\text { Flux } \\
4.5 \mu m \\
(\mathrm{mJy})\end{array}$ & $\begin{array}{l}\text { Error } \\
4.5 \mu m \\
(\mathrm{mJy})\end{array}$ & $\begin{array}{c}\text { Flux } \\
5.8 \mu m \\
(\mathrm{mJy})\end{array}$ & $\begin{array}{r}\text { Error } \\
5.8 \mu m \\
(\mathrm{mJy})\end{array}$ & $\begin{array}{c}\text { Flux } \\
8.0 \mu m \\
(\mathrm{mJy})\end{array}$ & $\begin{array}{l}\text { Error } \\
8.0 \mu m \\
(\mathrm{mJy})\end{array}$ & $\begin{array}{l}\text { Flux } \\
24 \mu m \\
(\mathrm{mJy})\end{array}$ & $\begin{array}{l}\text { Error } \\
24 \mu m \\
(\mathrm{mJy})\end{array}$ & $\begin{array}{c}\text { Flux } \\
70 \mu m \\
(\mathrm{mJy})\end{array}$ & $\begin{array}{l}\text { Error } \\
70 \mu m \\
(\mathrm{mJy})\end{array}$ & $\begin{array}{l}\text { Flux } \\
160 \mu m \\
(\mathrm{mJy})\end{array}$ & $\begin{array}{l}\text { Error } \\
160 \mu m \\
(\mathrm{mJy})\end{array}$ \\
\hline Abell 478 & 5.03 & 0.25 & 3.34 & 0.17 & 2.46 & 0.12 & 2.35 & 0.12 & $1.59^{\mathrm{b}}$ & 0.16 & $35.86^{\mathrm{d}}$ & 7.28 & $48.36^{\mathrm{d}}$ & 9.67 \\
\hline Abell 496 & 33.00 & 1.65 & 19.72 & 0.99 & 15.18 & 0.76 & 9.34 & 0.47 & $\ldots$ & $\ldots$ & $\ldots$ & $\ldots$ & $\ldots$ & $\ldots$ \\
\hline Abell $520^{\mathrm{e}}$ & $\ldots$ & $\ldots$ & $\ldots$ & $\ldots$ & $\ldots$ & $\ldots$ & $\ldots$ & $\ldots$ & $\ldots$ & $\ldots$ & $\ldots$ & $\ldots$ & $\ldots$ & $\ldots$ \\
\hline Abell 521 & $\ldots$ & $\ldots$ & $\ldots$ & $\ldots$ & $\ldots$ & $\ldots$ & $\ldots$ & $\ldots$ & 4.90 & 0.49 & $\ldots$ & $\ldots$ & $\ldots$ & $\ldots$ \\
\hline Abell 539 & $\ldots$ & $\ldots$ & $\ldots$ & $\ldots$ & $\ldots$ & $\ldots$ & $\ldots$ & $\ldots$ & $\ldots$ & $\ldots$ & $\ldots$ & $\ldots$ & $\ldots$ & $\ldots$ \\
\hline Abell 562 & $\ldots$ & $\ldots$ & $\ldots$ & $\ldots$ & $\ldots$ & $\ldots$ & $\ldots$ & $\ldots$ & $\ldots$ & $\ldots$ & $\ldots$ & $\ldots$ & $\ldots$ & $\ldots$ \\
\hline Abell 576 & $\ldots$ & $\ldots$ & $\ldots$ & $\ldots$ & $\ldots$ & $\ldots$ & $\cdots$ & $\ldots$ & $\ldots$ & $\ldots$ & $\ldots$ & $\ldots$ & $\cdots$ & $\ldots$ \\
\hline Abell 586 & $\ldots$ & $\ldots$ & $\ldots$ & $\ldots$ & $\ldots$ & $\ldots$ & $\ldots$ & $\ldots$ & 0.70 & $0.09^{\mathrm{b}}$ & $\ldots$ & $\ldots$ & $\ldots$ & $\ldots$ \\
\hline Abell 611 & $\ldots$ & $\ldots$ & $\ldots$ & $\ldots$ & $\ldots$ & $\ldots$ & $\ldots$ & $\ldots$ & 0.28 & 0.23 & $\ldots$ & $\cdots$ & $\cdots$ & $\cdots$ \\
\hline Abell 644 & $\ldots$ & $\ldots$ & $\ldots$ & $\ldots$ & $\ldots$ & $\ldots$ & $\ldots$ & $\ldots$ & $\ldots$ & $\ldots$ & $\ldots$ & $\ldots$ & $\ldots$ & $\ldots$ \\
\hline Abell 665 & $\ldots$ & $\ldots$ & $\ldots$ & $\ldots$ & $\ldots$ & $\ldots$ & $\ldots$ & $\ldots$ & 0.08 & 0.20 & $\ldots$ & $\ldots$ & $\cdots$ & $\ldots$ \\
\hline Abell 697 & 1.27 & 0.06 & 1.03 & 0.05 & 0.61 & 0.03 & 0.40 & 0.02 & 0.38 & 0.04 & $\cdots$ & $\cdots$ & $\cdots$ & $\cdots$ \\
\hline Abell 744 & $\ldots$ & $\ldots$ & $\ldots$ & $\ldots$ & $\ldots$ & $\ldots$ & $\ldots$ & $\ldots$ & $\ldots$ & $\ldots$ & $\ldots$ & $\ldots$ & $\ldots$ & $\ldots$ \\
\hline Abell 754 & $\ldots$ & $\ldots$ & $\ldots$ & $\ldots$ & $\ldots$ & $\ldots$ & $\ldots$ & $\ldots$ & $\ldots$ & $\ldots$ & $\ldots$ & $\ldots$ & $\ldots$ & $\ldots$ \\
\hline Abell 773 & 1.28 & 0.06 & 0.99 & 0.05 & 0.59 & 0.03 & 0.38 & 0.02 & 0.30 & 0.04 & $\ldots$ & $\ldots$ & $\ldots$ & $\ldots$ \\
\hline Abell 907 & $\ldots$ & $\ldots$ & $\ldots$ & $\ldots$ & $\ldots$ & $\ldots$ & $\ldots$ & $\ldots$ & $\ldots$ & $\ldots$ & $\cdots$ & $\ldots$ & $\cdots$ & $\cdots$ \\
\hline Abell 963 & 1.71 & 0.09 & 1.32 & 0.07 & 0.76 & 0.04 & 0.54 & 0.03 & $\ldots$ & $\ldots$ & $\ldots$ & $\ldots$ & $\ldots$ & $\ldots$ \\
\hline Abell 1060 & $\ldots$ & & $\ldots$ & $\ldots$ & $\ldots$ & $\ldots$ & $\ldots$ & $\ldots$ & $\ldots$ & $\ldots$ & $\ldots$ & $\ldots$ & $\ldots$ & $\ldots$ \\
\hline Abell 1063S & 1.09 & 0.05 & 0.87 & 0.04 & 0.54 & 0.03 & 0.34 & 0.02 & 0.06 & 0.01 & $\cdots$ & $\ldots$ & $\cdots$ & $\ldots$ \\
\hline Abell 1068 & 3.15 & 0.16 & 2.77 & 0.14 & 3.40 & 0.17 & 8.50 & 0.43 & $74.78^{\mathrm{c}}$ & 7.48 & 894.50 & 179.71 & $\ldots$ & $\ldots$ \\
\hline Abell 1201 & $\ldots$ & $\ldots$ & $\ldots$ & $\ldots$ & $\ldots$ & $\ldots$ & $\ldots$ & $\ldots$ & $\ldots$ & $\ldots$ & $\ldots$ & $\ldots$ & $\ldots$ & $\ldots$ \\
\hline Abell 1204 & 1.03 & 0.05 & 0.79 & 0.04 & 0.50 & 0.02 & 0.42 & 0.02 & 1.47 & 0.16 & $26.06^{\mathrm{d}}$ & 5.40 & $\ldots$ & $\ldots$ \\
\hline Abell 1240 & $\ldots$ & $\ldots$ & $\ldots$ & $\ldots$ & $\ldots$ & $\ldots$ & $\ldots$ & $\ldots$ & $\ldots$ & $\ldots$ & $\ldots$ & $\ldots$ & $\cdots$ & $\ldots$ \\
\hline Abell 1361 & 2.61 & 0.13 & 1.81 & 0.09 & 1.14 & 0.06 & 0.80 & 0.04 & $0.66^{\mathrm{b}}$ & 0.08 & $20.78^{\mathrm{d}}$ & 4.48 & $\ldots$ & $\ldots$ \\
\hline Abell 1413 & $\ldots$ & $\ldots$ & $\ldots$ & $\ldots$ & $\ldots$ & $\ldots$ & $\ldots$ & $\ldots$ & $\ldots$ & $\ldots$ & $\ldots$ & $\ldots$ & $\cdots$ & $\cdots$ \\
\hline Abell 1423 & $\ldots$ & $\ldots$ & $\cdots$ & $\cdots$ & $\ldots$ & $\ldots$ & $\ldots$ & $\ldots$ & $\ldots$ & $\ldots$ & $\ldots$ & $\ldots$ & $\ldots$ & $\ldots$ \\
\hline Abell 1446 & $\ldots$ & $\ldots$ & $\ldots$ & $\ldots$ & $\ldots$ & $\ldots$ & $\ldots$ & $\ldots$ & $\ldots$ & $\ldots$ & $\ldots$ & $\ldots$ & $\ldots$ & $\ldots$ \\
\hline Abell 1569 & $\ldots$ & $\ldots$ & $\ldots$ & $\ldots$ & $\ldots$ & $\ldots$ & $\ldots$ & $\ldots$ & $\ldots$ & $\ldots$ & $\ldots$ & $\ldots$ & $\ldots$ & $\ldots$ \\
\hline Abell 1576 & $\ldots$ & $\ldots$ & $\ldots$ & $\ldots$ & $\ldots$ & $\ldots$ & $\ldots$ & $\ldots$ & $\ldots$ & $\ldots$ & $\ldots$ & $\ldots$ & $\ldots$ & $\ldots$ \\
\hline
\end{tabular}


Table 5-Continued

\begin{tabular}{|c|c|c|c|c|c|c|c|c|c|c|c|c|c|c|}
\hline $\begin{array}{c}\text { Name } \\
\text { Cluster Name }\end{array}$ & $\begin{array}{c}\text { Flux } \\
3.6 \mu m \\
(\mathrm{mJy})\end{array}$ & $\begin{array}{r}\text { Error } \\
3.6 \mu m \\
(\mathrm{mJy})\end{array}$ & $\begin{array}{c}\text { Flux } \\
4.5 \mu m \\
(\mathrm{mJy})\end{array}$ & $\begin{array}{r}\text { Error } \\
4.5 \mu m \\
(\mathrm{mJy})\end{array}$ & $\begin{array}{c}\text { Flux } \\
5.8 \mu m \\
(\mathrm{mJy})\end{array}$ & $\begin{array}{r}\text { Error } \\
5.8 \mu m \\
(\mathrm{mJy})\end{array}$ & $\begin{array}{c}\text { Flux } \\
8.0 \mu m \\
(\mathrm{mJy})\end{array}$ & $\begin{array}{l}\text { Error } \\
8.0 \mu m \\
(\mathrm{mJy})\end{array}$ & $\begin{array}{l}\text { Flux } \\
24 \mu m \\
(\mathrm{mJy})\end{array}$ & $\begin{array}{l}\text { Error } \\
24 \mu m \\
(\mathrm{mJy})\end{array}$ & $\begin{array}{l}\text { Flux } \\
70 \mu m \\
(\mathrm{mJy})\end{array}$ & $\begin{array}{l}\text { Error } \\
70 \mu m \\
(\mathrm{mJy})\end{array}$ & $\begin{array}{l}\text { Flux } \\
160 \mu m \\
\text { (mJy) }\end{array}$ & $\begin{array}{l}\text { Error } \\
160 \mu m \\
(\mathrm{mJy})\end{array}$ \\
\hline Abell 1644 & 2.24 & 0.11 & 1.58 & 0.08 & 1.27 & 0.06 & 2.49 & 0.12 & & $\ldots$ & $\ldots$ & $\ldots$ & $\cdots$ & \\
\hline Abell 1650 & $\ldots$ & $\ldots$ & $\ldots$ & $\ldots$ & $\ldots$ & $\ldots$ & $\ldots$ & $\ldots$ & $\ldots$ & $\ldots$ & $\ldots$ & $\ldots$ & $\cdots$ & $\ldots$ \\
\hline Abell 1651 & $\ldots$ & $\ldots$ & $\ldots$ & $\ldots$ & $\ldots$ & $\ldots$ & $\ldots$ & $\ldots$ & $\ldots$ & $\ldots$ & $\ldots$ & $\ldots$ & $\ldots$ & $\ldots$ \\
\hline Abell 1664 & $\ldots$ & $\ldots$ & $\ldots$ & $\ldots$ & $\ldots$ & $\ldots$ & $\ldots$ & $\ldots$ & 4.08 & 0.41 & 85.52 & 23.12 & $\ldots$ & $\ldots$ \\
\hline Abell 1689 & 1.35 & 0.07 & 1.01 & 0.05 & 0.59 & 0.03 & 0.39 & 0.02 & $0.92^{\mathrm{b}}$ & 0.09 & 1.74 & 0 & 4.43 & 0 \\
\hline Abell 1736 & $\ldots$ & $\ldots$ & $\ldots$ & $\ldots$ & $\ldots$ & $\ldots$ & $\ldots$ & $\ldots$ & $\ldots$ & $\ldots$ & $\ldots$ & $\ldots$ & $\ldots$ & $\ldots$ \\
\hline Abell 1758 & 0.80 & 0.04 & 0.65 & 0.03 & 0.38 & 0.02 & 0.23 & 0.01 & 0.23 & 0.03 & $\ldots$ & $\ldots$ & $\ldots$ & $\ldots$ \\
\hline Abell 1763 & 1.45 & 0.07 & 1.12 & 0.06 & 0.61 & 0.03 & 0.36 & 0.02 & 0.33 & 0.10 & 8.07 & 0 & 28.28 & 0 \\
\hline Abell 1795 & $\ldots$ & $\ldots$ & $\ldots$ & $\ldots$ & $\ldots$ & $\ldots$ & $\ldots$ & $\ldots$ & 2.17 & 0.25 & 37.21 & 0 & $\ldots$ & \\
\hline Abell 1835 & 1.93 & 0.10 & 1.83 & 0.09 & 1.29 & 0.06 & 4.53 & 0.23 & $17.80^{\mathrm{C}}$ & c 1.78 & 175.07 & 36.14 & 317.29 & 64.16 \\
\hline Abell 1914 & 0.98 & 0.05 & 0.70 & 0.04 & 0.39 & 0.02 & 0.26 & 0.01 & $0.12^{\mathrm{b}}$ & 0.01 & $\ldots$ & $\ldots$ & $\ldots$ & $\ldots$ \\
\hline Abell 1942 & $\ldots$ & $\ldots$ & $\ldots$ & $\ldots$ & $\ldots$ & $\ldots$ & $\ldots$ & $\ldots$ & $\ldots$ & $\ldots$ & $\ldots$ & $\ldots$ & $\ldots$ & $\ldots$ \\
\hline Abell 1991 & 8.99 & 0.45 & 5.64 & 0.28 & 3.88 & 0.19 & 2.54 & 0.13 & $\ldots$ & $\ldots$ & $\ldots$ & $\ldots$ & $\ldots$ & $\ldots$ \\
\hline Abell 1995 & $\ldots$ & $\ldots$ & $\ldots$ & $\ldots$ & $\ldots$ & $\ldots$ & $\ldots$ & $\ldots$ & $\ldots$ & $\ldots$ & $\ldots$ & $\ldots$ & $\ldots$ & $\ldots$ \\
\hline Abell 2029 & $\ldots$ & $\ldots$ & $\ldots$ & $\ldots$ & $\ldots$ & $\ldots$ & $\ldots$ & $\ldots$ & 1.07 & 0.19 & 14.88 & 0 & 46.57 & 0 \\
\hline Abell 2034 & $\ldots$ & $\ldots$ & $\ldots$ & $\ldots$ & $\ldots$ & $\ldots$ & $\ldots$ & $\ldots$ & $\ldots$ & $\ldots$ & $\ldots$ & $\ldots$ & $\ldots$ & $\ldots$ \\
\hline Abell 2052 & 25.15 & 1.26 & 15.23 & 0.76 & 11.58 & 0.58 & 7.85 & 0.39 & 4.91 & 0.49 & $28.97^{\mathrm{d}}$ & 6.08 & $\ldots$ & $\ldots$ \\
\hline Abell 2063 & $\ldots$ & $\ldots$ & $\ldots$ & $\ldots$ & $\ldots$ & $\ldots$ & $\ldots$ & $\ldots$ & 1.11 & 0.13 & $14.61^{\mathrm{a}}$ & 0 & $\ldots$ & $\ldots$ \\
\hline Abell 2065 & $\cdots$ & $\cdots$ & $\cdots$ & $\ldots$ & $\cdots$ & $\cdots$ & $\cdots$ & $\cdots$ & $\ldots$ & $\ldots$ & $\ldots$ & $\cdots$ & $\cdots$ & $\cdots$ \\
\hline Abell 2069 & $\ldots$ & $\ldots$ & $\ldots$ & $\ldots$ & $\ldots$ & $\ldots$ & $\ldots$ & $\ldots$ & $\ldots$ & $\ldots$ & $\cdots$ & $\ldots$ & $\ldots$ & $\ldots$ \\
\hline Abell 2104 & 1.42 & 0.07 & 1.07 & 0.05 & 0.66 & 0.03 & 0.42 & 0.02 & $\ldots$ & $\ldots$ & $\ldots$ & $\ldots$ & $\ldots$ & $\ldots$ \\
\hline Abell 2107 & $\ldots$ & $\ldots$ & $\ldots$ & $\ldots$ & $\ldots$ & $\ldots$ & $\ldots$ & $\ldots$ & $1.56^{\mathrm{b}}$ & 0.16 & $6.23^{\mathrm{a}}$ & 0 & $\ldots$ & $\ldots$ \\
\hline Abell 2111 & $\ldots$ & $\ldots$ & $\ldots$ & $\ldots$ & $\ldots$ & $\ldots$ & $\ldots$ & $\ldots$ & $\ldots$ & $\ldots$ & $\ldots$ & $\ldots$ & $\ldots$ & $\ldots$ \\
\hline Abell 2124 & $\ldots$ & $\ldots$ & $\ldots$ & $\ldots$ & $\ldots$ & $\ldots$ & $\ldots$ & $\ldots$ & $\ldots$ & $\ldots$ & $\ldots$ & $\ldots$ & $\ldots$ & $\ldots$ \\
\hline Abell 2125 & $\ldots$ & $\ldots$ & $\ldots$ & $\ldots$ & $\ldots$ & $\ldots$ & $\ldots$ & $\ldots$ & $0.28^{\mathrm{b}}$ & 0.03 & $\ldots$ & $\ldots$ & $\ldots$ & $\ldots$ \\
\hline Abell 2142 & $\ldots$ & $\ldots$ & $\ldots$ & $\ldots$ & $\ldots$ & $\ldots$ & $\ldots$ & $\ldots$ & $0.54^{\mathrm{b}}$ & 0.08 & $8.38^{\mathrm{a}}$ & 0 & $\ldots$ & $\ldots$ \\
\hline Abell 2147 & $\ldots$ & $\ldots$ & $\ldots$ & $\ldots$ & $\ldots$ & $\ldots$ & $\ldots$ & $\ldots$ & $\ldots$ & $\ldots$ & $\ldots$ & $\ldots$ & $\ldots$ & $\cdots$ \\
\hline Abell 2151 & $\ldots$ & $\ldots$ & $\ldots$ & $\ldots$ & $\ldots$ & $\ldots$ & $\ldots$ & $\ldots$ & $0.93^{\mathrm{b}}$ & 0.13 & 22.08 & 0 & 65.70 & 0 \\
\hline Abell 2163 & $\ldots$ & $\ldots$ & $\ldots$ & $\ldots$ & $\ldots$ & $\ldots$ & $\ldots$ & $\ldots$ & $\ldots$ & $\ldots$ & $\ldots$ & $\ldots$ & $\ldots$ & $\ldots$ \\
\hline
\end{tabular}


Table 5-Continued

\begin{tabular}{|c|c|c|c|c|c|c|c|c|c|c|c|c|c|c|}
\hline $\begin{array}{c}\text { Name } \\
\text { Cluster Name }\end{array}$ & $\begin{array}{c}\text { Flux } \\
3.6 \mu m \\
(\mathrm{mJy})\end{array}$ & $\begin{array}{c}\text { Error } \\
3.6 \mu m \\
(\mathrm{mJy})\end{array}$ & $\begin{array}{l}\text { Flux } \\
4.5 \mu m \\
\text { (mJy) }\end{array}$ & $\begin{array}{r}\text { Error } \\
4.5 \mu m \\
(\mathrm{mJy})\end{array}$ & $\begin{array}{c}\text { Flux } \\
5.8 \mu m \\
(\mathrm{mJy})\end{array}$ & $\begin{array}{l}\text { Error } \\
5.8 \mu m \\
(\mathrm{mJy})\end{array}$ & $\begin{array}{c}\text { Flux } \\
8.0 \mu m \\
(\mathrm{mJy})\end{array}$ & $\begin{array}{l}\text { Error } \\
8.0 \mu m \\
(\mathrm{mJy})\end{array}$ & $\begin{array}{c}\text { Flux } \\
24 \mu m \\
(\mathrm{mJy})\end{array}$ & $\begin{array}{l}\text { Error } \\
24 \mu m \\
(\mathrm{mJy})\end{array}$ & $\begin{array}{c}\text { Flux } \\
70 \mu m \\
(\mathrm{mJy})\end{array}$ & $\begin{array}{l}\text { Error } \\
70 \mu m \\
(\mathrm{mJy})\end{array}$ & $\begin{array}{l}\text { Flux } \\
160 \mu m \\
(\mathrm{mJy})\end{array}$ & $\begin{array}{l}\text { Error } \\
160 \mu m \\
(\mathrm{mJy})\end{array}$ \\
\hline Abell 2187 & $\cdots$ & $\cdots$ & $\cdots$ & $\cdots$ & $\cdots$ & $\ldots$ & $\cdots$ & $\cdots$ & $\cdots$ & $\cdots$ & $\cdots$ & $\cdots$ & $\cdots$ & $\cdots$ \\
\hline Abell $2199^{\mathrm{e}}$ & 46.60 & 2.33 & 28.35 & 1.42 & 21.31 & 1.07 & 14.38 & 0.72 & $5.06^{\mathrm{f}}$ & 0.51 & $\ldots$ & $\cdots$ & 173.77 & 37.61 \\
\hline Abell 2204 & 1.71 & 0.09 & 1.19 & 0.06 & 0.76 & 0.04 & 0.68 & 0.03 & 2.46 & 0.25 & $47.57^{\mathrm{d}}$ & 9.92 & $\ldots$ & $\ldots$ \\
\hline Abell 2218 & 1.12 & 0.06 & 0.82 & 0.04 & 0.48 & 0.02 & 0.32 & 0.02 & 0.05 & 0 & 46.12 & 0 & 46.57 & 0 \\
\hline Abell 2219 & 1.00 & 0.05 & 0.77 & 0.04 & 0.43 & 0.02 & 0.29 & 0.01 & 0.29 & 0.03 & $\ldots$ & $\cdots$ & $\ldots$ & $\cdots$ \\
\hline Abell 2244 & $\cdots$ & $\ldots$ & $\cdots$ & $\cdots$ & $\cdots$ & $\cdots$ & $\ldots$ & & $\cdots$ & $\ldots$ & $\ldots$ & $\cdots$ & $\cdots$ & $\cdots$ \\
\hline Abell 2255 & $\cdots$ & $\cdots$ & $\cdots$ & $\cdots$ & $\cdots$ & $\cdots$ & $\cdots$ & $\cdots$ & 0.31 & 0.05 & $9.03^{\mathrm{a}}$ & 0 & $\cdots$ & $\cdots$ \\
\hline Abell 2256 & $\ldots$ & $\ldots$ & $\ldots$ & $\ldots$ & $\ldots$ & $\ldots$ & $\ldots$ & $\ldots$ & $\ldots$ & $\ldots$ & $\ldots$ & $\ldots$ & $\ldots$ & $\ldots$ \\
\hline Abell 2259 & $\ldots$ & $\cdots$ & $\ldots$ & $\ldots$ & $\ldots$ & $\ldots$ & $\ldots$ & $\ldots$ & $\ldots$ & $\ldots$ & $\ldots$ & $\ldots$ & $\ldots$ & $\cdots$ \\
\hline Abell 2261 & 2.03 & 0.10 & 1.57 & 0.08 & 0.87 & 0.04 & 0.60 & 0.03 & 0.44 & 0.05 & 3.29 & 0 & $38.02^{\mathrm{a}}$ & 0 \\
\hline Abell 2294 & $\ldots$ & $\ldots$ & $\ldots$ & $\ldots$ & $\ldots$ & $\ldots$ & $\ldots$ & & $\ldots$ & $\ldots$ & $\ldots$ & $\ldots$ & $\ldots$ & $\cdots$ \\
\hline Abell 2319 & $\ldots$ & $\cdots$ & $\cdots$ & $\cdots$ & $\cdots$ & $\cdots$ & $\cdots$ & $\cdots$ & $\ldots$ & $\ldots$ & $\cdots$ & $\ldots$ & $\cdots$ & $\cdots$ \\
\hline Abell 2384 & $\ldots$ & $\ldots$ & $\ldots$ & $\ldots$ & $\ldots$ & $\ldots$ & $\ldots$ & $\ldots$ & $\ldots$ & $\ldots$ & $\ldots$ & $\ldots$ & $\ldots$ & \\
\hline Abell 2390 & 1.01 & 0.05 & 0.80 & 0.04 & 0.50 & 0.03 & 0.62 & 0.03 & $1.23^{\mathrm{c}}$ & 0.12 & 48.80 & 12.59 & 120.01 & 24.00 \\
\hline Abell 2409 & $\cdots$ & $\cdots$ & $\cdots$ & $\cdots$ & $\cdots$ & $\ldots$ & $\ldots$ & $\ldots$ & $\cdots$ & $\ldots$ & $\ldots$ & $\ldots$ & $\ldots$ & $\ldots$ \\
\hline Abell 2420 & $\ldots$ & $\cdots$ & $\cdots$ & $\ldots$ & $\cdots$ & $\cdots$ & $\cdots$ & $\cdots$ & $\ldots$ & $\ldots$ & $\cdots$ & $\ldots$ & $\cdots$ & . \\
\hline Abell 2462 & $\ldots$ & $\ldots$ & $\ldots$ & $\ldots$ & $\ldots$ & $\cdots$ & $\ldots$ & $\ldots$ & $\ldots$ & $\ldots$ & $\ldots$ & $\ldots$ & $\ldots$ & $\ldots$ \\
\hline Abell 2537 & $\ldots$ & $\cdots$ & $\cdots$ & $\cdots$ & $\cdots$ & $\cdots$ & $\cdots$ & $\cdots$ & 0.04 & 0.02 & $\cdots$ & $\cdots$ & $\cdots$ & $\cdots$ \\
\hline Abell 2554 & $\ldots$ & $\cdots$ & $\ldots$ & $\cdots$ & $\cdots$ & $\cdots$ & $\cdots$ & $\ldots$ & $\cdots$ & $\ldots$ & $\ldots$ & $\cdots$ & $\cdots$ & $\ldots$ \\
\hline Abell 2556 & $\cdots$ & $\ldots$ & $\ldots$ & $\cdots$ & $\cdots$ & $\cdots$ & $\cdots$ & $\cdots$ & $\ldots$ & $\cdots$ & $\cdots$ & $\ldots$ & $\cdots$ & $\cdots$ \\
\hline Abell 2589 & $\ldots$ & $\ldots$ & $\ldots$ & $\ldots$ & $\ldots$ & $\ldots$ & $\ldots$ & $\ldots$ & $\ldots$ & $\ldots$ & $\ldots$ & $\ldots$ & $\ldots$ & $\ldots$ \\
\hline Abell 2597 & 3.69 & 0.18 & 2.48 & 0.12 & 2.05 & 0.10 & 2.05 & 0.10 & $2.36^{\mathrm{c}}$ & 0.25 & $62.87^{\mathrm{d}}$ & 19.09 & 42.05 & 10.28 \\
\hline Abell 2626 & 10.76 & 0.54 & 6.80 & 0.34 & 4.85 & 0.24 & 3.00 & 0.15 & $1.34^{\mathrm{b}}$ & 0.14 & 5.96 & 0 & $\ldots$ & $\ldots$ \\
\hline Abell 2631 & $\ldots$ & $\cdots$ & $\cdots$ & $\cdots$ & $\cdots$ & $\cdots$ & $\cdots$ & $\cdots$ & $\cdots$ & $\cdots$ & $\cdots$ & $\cdots$ & $\cdots$ & $\cdots$ \\
\hline Abell 2657 & $\cdots$ & $\cdots$ & $\cdots$ & $\ldots$ & $\cdots$ & $\ldots$ & $\cdots$ & $\ldots$ & $\ldots$ & $\cdots$ & $\cdots$ & $\cdots$ & $\cdots$ & \\
\hline Abell 2667 & 0.76 & 0.04 & 0.61 & 0.03 & 0.46 & 0.02 & 0.62 & 0.03 & $8.19^{f}$ & 0.82 & $\ldots$ & $\ldots$ & $\ldots$ & $\cdots$ \\
\hline Abell 2717 & $\ldots$ & $\ldots$ & $\ldots$ & $\ldots$ & $\ldots$ & $\ldots$ & $\ldots$ & $\ldots$ & $\ldots$ & $\ldots$ & $\ldots$ & $\ldots$ & $\ldots$ & $\ldots$ \\
\hline Abell 2744a & 0.65 & 0.03225 & 0.52 & 0.03 & 0.32 & 0.02 & 0.21 & 0.01 & $0.48^{\mathrm{b}}$ & 0.05 & $4.29^{\mathrm{a}}$ & 0 & $23.63^{\mathrm{a}}$ & 0 \\
\hline Abell 2744b & 0.64 & 0.03 & 0.52 & 0.03 & 0.32 & 0.02 & 0.20 & 0.01 & 0.09 & 0 & 2.34 & 0 & 6.99 & 0 \\
\hline
\end{tabular}


Table 5-Continued

\begin{tabular}{|c|c|c|c|c|c|c|c|c|c|c|c|c|c|c|}
\hline $\begin{array}{c}\text { Name } \\
\text { Cluster Name }\end{array}$ & $\begin{array}{l}\text { Flux } \\
3.6 \mu m \\
(\mathrm{mJy})\end{array}$ & $\begin{array}{r}\text { Error } \\
3.6 \mu m \\
(\mathrm{mJy})\end{array}$ & $\begin{array}{c}\text { Flux } \\
4.5 \mu m \\
(\mathrm{mJy})\end{array}$ & $\begin{array}{l}\text { Error } \\
4.5 \mu m \\
(\mathrm{mJy})\end{array}$ & $\begin{array}{c}\text { Flux } \\
5.8 \mu m \\
(\mathrm{mJy})\end{array}$ & $\begin{array}{l}\text { Error } \\
5.8 \mu m \\
(\mathrm{mJy})\end{array}$ & $\begin{array}{c}\text { Flux } \\
8.0 \mu m \\
(\mathrm{mJy})\end{array}$ & $\begin{array}{l}\text { Error } \\
8.0 \mu m \\
(\mathrm{mJy})\end{array}$ & $\begin{array}{l}\text { Flux } \\
24 \mu m \\
(\mathrm{mJy})\end{array}$ & $\begin{array}{l}\text { Error } \\
24 \mu m \\
(\mathrm{mJy})\end{array}$ & $\begin{array}{l}\text { Flux } \\
70 \mu m \\
(\mathrm{mJy})\end{array}$ & $\begin{array}{c}\text { Error } \\
70 \mu m \\
(\mathrm{mJy})\end{array}$ & $\begin{array}{l}\text { Flux } \\
160 \mu m \\
(\mathrm{mJy})\end{array}$ & $\begin{array}{l}\text { Error } \\
160 \mu m \\
(\mathrm{mJy})\end{array}$ \\
\hline Abell 2813 & $\cdots$ & $\cdots$ & $\cdots$ & $\ldots$ & $\ldots$ & $\cdots$ & $\ldots$ & $\ldots$ & $\ldots$ & $\ldots$ & $\ldots$ & $\ldots$ & $\ldots$ & $\ldots$ \\
\hline Abell 3084 & $\ldots$ & $\cdots$ & $\ldots$ & $\ldots$ & $\ldots$ & $\ldots$ & $\cdots$ & $\ldots$ & 0.41 & 0.07 & $\ldots$ & $\ldots$ & $\ldots$ & $\ldots$ \\
\hline Abell 3088 & $\ldots$ & $\ldots$ & $\ldots$ & $\ldots$ & $\ldots$ & $\ldots$ & $\ldots$ & $\ldots$ & $0.40^{\mathrm{b}}$ & 0.05 & $\ldots$ & $\ldots$ & $\ldots$ & $\ldots$ \\
\hline Abell 3112 & 7.80 & 0.39 & 5.12 & 0.26 & 3.53 & 0.18 & 2.92 & 0.15 & 3.07 & 0.31 & $5.96^{\mathrm{a}}$ & 0 & $\ldots$ & $\ldots$ \\
\hline Abell 3120 & $\ldots$ & $\ldots$ & $\ldots$ & $\ldots$ & $\ldots$ & $\ldots$ & $\ldots$ & $\ldots$ & $\ldots$ & $\ldots$ & $\ldots$ & $\ldots$ & $\ldots$ & $\ldots$ \\
\hline Abell 3158 & $\ldots$ & $\ldots$ & $\ldots$ & $\ldots$ & $\ldots$ & $\ldots$ & $\ldots$ & $\ldots$ & $0.70^{\mathrm{b}}$ & 0.10 & 21.59 & 0 & 40.12 & 0 \\
\hline Abell 3266 & $\ldots$ & $\ldots$ & $\ldots$ & $\ldots$ & $\ldots$ & $\ldots$ & $\ldots$ & $\ldots$ & $2.64^{\mathrm{b}}$ & 0.30 & 14.88 & 0 & 42.60 & 0 \\
\hline Abell 3364 & $\ldots$ & $\ldots$ & $\ldots$ & $\ldots$ & $\ldots$ & $\ldots$ & $\ldots$ & $\ldots$ & 0.18 & 0.06 & $\ldots$ & $\ldots$ & $\ldots$ & $\ldots$ \\
\hline Abell 3376 & $\ldots$ & $\ldots$ & $\ldots$ & $\ldots$ & $\ldots$ & $\ldots$ & $\ldots$ & $\ldots$ & $\ldots$ & $\ldots$ & $\ldots$ & $\ldots$ & $\ldots$ & $\ldots$ \\
\hline Abell 3391 & $\ldots$ & $\ldots$ & $\ldots$ & $\ldots$ & $\ldots$ & $\ldots$ & $\ldots$ & $\ldots$ & 1.75 & 0.18 & 1.31 & 0 & $25.24^{\mathrm{a}}$ & 0 \\
\hline Abell 3395 & $\cdots$ & $\ldots$ & $\cdots$ & $\ldots$ & $\ldots$ & $\ldots$ & $\ldots$ & $\cdots$ & $\ldots$ & $\ldots$ & $\ldots$ & $\ldots$ & $\ldots$ & $\ldots$ \\
\hline Abell 3528S & $\ldots$ & $\cdots$ & $\ldots$ & $\ldots$ & $\ldots$ & $\ldots$ & $\ldots$ & $\ldots$ & $\ldots$ & $\ldots$ & $\ldots$ & $\ldots$ & $\ldots$ & $\ldots$ \\
\hline Abell 3558 & $\ldots$ & $\ldots$ & $\ldots$ & $\ldots$ & $\ldots$ & $\ldots$ & $\ldots$ & $\ldots$ & $\ldots$ & $\ldots$ & $\ldots$ & $\ldots$ & $\ldots$ & $\ldots$ \\
\hline Abell 3562 & $\cdots$ & $\cdots$ & $\cdots$ & $\cdots$ & $\cdots$ & $\ldots$ & $\ldots$ & $\cdots$ & $\ldots$ & $\cdots$ & $\cdots$ & $\cdots$ & $\cdots$ & $\ldots$ \\
\hline Abell 3571 & $\cdots$ & $\cdots$ & $\cdots$ & $\cdots$ & $\cdots$ & $\cdots$ & $\cdots$ & $\cdots$ & $\ldots$ & $\cdots$ & $\ldots$ & $\cdots$ & $\cdots$ & $\ldots$ \\
\hline Abell 3581 & $\ldots$ & $\ldots$ & $\ldots$ & $\ldots$ & $\ldots$ & $\ldots$ & $\ldots$ & $\ldots$ & $\ldots$ & $\ldots$ & $\ldots$ & $\ldots$ & $\ldots$ & $\ldots$ \\
\hline Abell 3667 & $\ldots$ & $\ldots$ & $\ldots$ & $\ldots$ & $\ldots$ & $\ldots$ & $\ldots$ & $\ldots$ & $\ldots$ & $\ldots$ & $\ldots$ & $\ldots$ & $\ldots$ & $\ldots$ \\
\hline Abell 3822 & $\ldots$ & $\ldots$ & $\ldots$ & $\ldots$ & $\ldots$ & $\ldots$ & $\cdots$ & $\ldots$ & $\ldots$ & $\ldots$ & $\ldots$ & $\ldots$ & $\ldots$ & $\ldots$ \\
\hline Abell 3827 & $\cdots$ & $\cdots$ & $\cdots$ & $\cdots$ & $\cdots$ & $\cdots$ & $\cdots$ & $\cdots$ & $\cdots$ & $\cdots$ & $\cdots$ & $\cdots$ & $\cdots$ & $\cdots$ \\
\hline Abell 3921 & $\cdots$ & $\ldots$ & $\ldots$ & $\ldots$ & $\ldots$ & $\ldots$ & $\cdots$ & $\ldots$ & $\ldots$ & $\ldots$ & $\ldots$ & $\cdots$ & $\cdots$ & $\ldots$ \\
\hline Abell 4038 & $\ldots$ & $\ldots$ & $\ldots$ & $\ldots$ & $\ldots$ & $\ldots$ & $\ldots$ & $\ldots$ & $\ldots$ & $\ldots$ & $\ldots$ & $\ldots$ & $\ldots$ & $\ldots$ \\
\hline Abell 4059 & 18.05 & 0.90 & 11.23 & 0.56 & 7.91 & 0.40 & 5.78 & 0.29 & 1.83 & 0.19 & $13.41^{\mathrm{d}}$ & 2.98 & $\ldots$ & $\ldots$ \\
\hline Abell S0405 & $\ldots$ & $\ldots$ & $\ldots$ & $\ldots$ & $\ldots$ & $\ldots$ & $\ldots$ & $\ldots$ & $\ldots$ & $\ldots$ & $\ldots$ & $\ldots$ & $\cdots$ & $\cdots$ \\
\hline Abell S0592 & $\ldots$ & $\ldots$ & $\ldots$ & $\ldots$ & $\ldots$ & $\ldots$ & $\ldots$ & $\ldots$ & 0.16 & 0.02 & $\cdots$ & $\ldots$ & $\cdots$ & $\ldots$ \\
\hline AC 114 & 0.93 & 0.05 & 0.75 & 0.04 & 0.46 & 0.02 & 0.31 & 0.02 & 0.10 & 0.01 & $\cdots$ & $\cdots$ & $\cdots$ & $\ldots$ \\
\hline AWM7 & $\ldots$ & $\ldots$ & $\ldots$ & $\ldots$ & $\ldots$ & $\ldots$ & $\ldots$ & $\ldots$ & $\ldots$ & $\ldots$ & $\ldots$ & $\ldots$ & $\ldots$ & $\ldots$ \\
\hline CENTAURUS & 328.00 & 16.40 & 191.00 & 9.55 & 162.00 & 8.10 & 100.00 & 5.00 & $25.60^{f}$ & 3.00 & 165.00 & 41.28 & 256.00 & 60.15 \\
\hline CID 0072 & 19.16 & 0.96 & 11.47 & 0.57 & 8.50 & 0.42 & 5.64 & 0.28 & $1.13^{\mathrm{b}}$ & 0.12 & $5.78^{\mathrm{a}}$ & 0 & $\ldots$ & $\ldots$ \\
\hline CL J1226.9+3332 & 20.48 & 0.02 & 0.33 & 0.02 & 0.25 & 0.01 & 0.14 & 0.01 & $0.20^{\mathrm{b}}$ & 0.03 & 3.66 & 0 & $30.13^{\mathrm{a}}$ & 0 \\
\hline
\end{tabular}


Table 5-Continued

\begin{tabular}{|c|c|c|c|c|c|c|c|c|c|c|c|c|c|c|}
\hline $\begin{array}{c}\text { Name } \\
\text { Cluster Name }\end{array}$ & $\begin{array}{l}\text { Flux } \\
3.6 \mu m \\
(\mathrm{mJy})\end{array}$ & $\begin{array}{l}\text { Error } \\
3.6 \mu m \\
(\mathrm{mJy})\end{array}$ & $\begin{array}{c}\text { Flux } \\
4.5 \mu m \\
(\mathrm{mJy})\end{array}$ & $\begin{array}{l}\text { Error } \\
4.5 \mu m \\
(\mathrm{mJy})\end{array}$ & $\begin{array}{c}\text { Flux } \\
5.8 \mu m \\
(\mathrm{mJy})\end{array}$ & $\begin{array}{l}\text { Error } \\
5.8 \mu m \\
(\mathrm{mJy})\end{array}$ & $\begin{array}{l}\text { Flux } \\
8.0 \mu m \\
(\mathrm{mJy})\end{array}$ & $\begin{array}{l}\text { Error } \\
8.0 \mu m \\
(\mathrm{mJy})\end{array}$ & $\begin{array}{l}\text { Flux } \\
24 \mu m \\
(\mathrm{mJy})\end{array}$ & $\begin{array}{l}\text { Error } \\
24 \mu m \\
(\mathrm{mJy})\end{array}$ & $\begin{array}{c}\text { Flux } \\
70 \mu m \\
(\mathrm{mJy})\end{array}$ & $\begin{array}{l}\text { Error } \\
70 \mu m \\
(\mathrm{mJy})\end{array}$ & $\begin{array}{l}\text { Flux } \\
160 \mu m \\
(\mathrm{mJy})\end{array}$ & $\begin{array}{l}\text { Error } \\
160 \mu m \\
(\mathrm{mJy})\end{array}$ \\
\hline CYGNUS A & $\cdots$ & $\cdots$ & 16.32 & 0.82 & $\cdots$ & $\cdots$ & 59.12 & 2.96 & 677.50 & 67.75 & 1394.46 & 280.16 & 454.29 & 94.03 \\
\hline ESO 3060170 & $\ldots$ & $\cdots$ & $\ldots$ & $\cdots$ & $\ldots$ & $\ldots$ & $\ldots$ & $\cdots$ & $\ldots$ & $\ldots$ & $\ldots$ & $\ldots$ & $\ldots$ & $\ldots$ \\
\hline ESO 5520200 & $\ldots$ & $\ldots$ & $\ldots$ & $\ldots$ & $\ldots$ & $\ldots$ & $\ldots$ & $\ldots$ & $\ldots$ & $\ldots$ & $\ldots$ & $\ldots$ & $\ldots$ & $\ldots$ \\
\hline EXO 0422-086 & $\ldots$ & $\ldots$ & $\ldots$ & $\ldots$ & $\ldots$ & $\ldots$ & $\ldots$ & $\ldots$ & $\ldots$ & $\ldots$ & $\ldots$ & $\ldots$ & $\ldots$ & $\ldots$ \\
\hline HCG 0062 & 115.00 & 5.75 & 68.24 & 3.41 & 52.61 & 2.63 & 74.77 & 3.74 & $13.28^{\mathrm{f}}$ & 1.36 & 24.05 & 0 & $\ldots$ & $\ldots$ \\
\hline HCG $42^{\mathrm{e}}$ & 158.20 & 7.91 & 92.28 & 4.61 & 77.33 & 3.87 & 45.45 & 2.27 & $12.40^{\mathrm{f}}$ & 1.31 & 8.58 & 0 & $\ldots$ & $\ldots$ \\
\hline HERCULES A & $\ldots$ & $\ldots$ & 0.91 & 0.05 & $\ldots$ & $\ldots$ & 0.43 & 0.02 & 0.17 & 0.02 & $\ldots$ & $\ldots$ & $\ldots$ & $\ldots$ \\
\hline HYDRA A & $\ldots$ & $\ldots$ & 7.74 & 0.39 & $\ldots$ & $\ldots$ & 6.86 & 0.34 & $9.15^{\mathrm{c}}$ & 0.92 & 155.18 & 36.56 & 181.77 & 38.98 \\
\hline $\mathrm{M} 49^{\mathrm{e}}$ & 2042.00 & 102.10 & 1195.00 & 59.75 & 564.00 & 28.20 & 754.00 & 37.70 & $924.50^{\mathrm{f}}$ & f 92.48 & 205.24 & 43.76 & 26.98 & 5.90 \\
\hline $\mathrm{M} 87^{\mathrm{e}}$ & 1502.00 & 75.10 & 890.50 & 44.53 & 905.80 & 45.29 & 493.30 & 24.67 & $256.17^{\mathrm{f}}$ & f 25.62 & 395.40 & 83.69 & 588.32 & 119.94 \\
\hline MACS J0011.7-1523 & $\ldots$ & $\ldots$ & $\ldots$ & $\ldots$ & $\ldots$ & $\ldots$ & $\ldots$ & $\ldots$ & $\ldots$ & $\ldots$ & $\ldots$ & $\ldots$ & $\ldots$ & $\ldots$ \\
\hline MACS J0035.4-2015 & $\ldots$ & $\ldots$ & $\ldots$ & $\ldots$ & $\ldots$ & $\cdots$ & $\ldots$ & $\ldots$ & $\ldots$ & $\ldots$ & $\ldots$ & $\ldots$ & $\ldots$ & $\ldots$ \\
\hline MACS J0159.8-0849 & $\ldots$ & $\ldots$ & $\ldots$ & $\ldots$ & $\ldots$ & $\ldots$ & $\ldots$ & $\ldots$ & $\ldots$ & $\ldots$ & $\ldots$ & $\ldots$ & $\ldots$ & $\ldots$ \\
\hline MACS J0242.5-2132 & $\cdots$ & $\cdots$ & $\cdots$ & $\cdots$ & $\cdots$ & $\cdots$ & $\ldots$ & $\cdots$ & $\ldots$ & $\cdots$ & $\cdots$ & $\cdots$ & $\cdots$ & $\cdots$ \\
\hline MACS J0257.1-2325 & $\cdots$ & $\cdots$ & $\cdots$ & $\cdots$ & $\ldots$ & $\ldots$ & $\cdots$ & $\ldots$ & $\ldots$ & $\cdots$ & $\cdots$ & $\ldots$ & $\ldots$ & $\ldots$ \\
\hline MACS J0257.6-2209 & $\ldots$ & $\ldots$ & $\ldots$ & $\ldots$ & $\ldots$ & $\ldots$ & $\ldots$ & $\cdots$ & $\ldots$ & $\ldots$ & $\ldots$ & $\ldots$ & $\ldots$ & $\ldots$ \\
\hline MACS J0308.9+2645 & $\cdots$ & $\cdots$ & $\cdots$ & $\cdots$ & $\cdots$ & $\cdots$ & $\cdots$ & $\cdots$ & $\ldots$ & $\ldots$ & $\ldots$ & $\ldots$ & $\ldots$ & $\ldots$ \\
\hline MACS J0329.6-0211 & $\cdots$ & $\cdots$ & $\ldots$ & $\cdots$ & $\cdots$ & $\cdots$ & $\cdots$ & $\cdots$ & $\ldots$ & $\ldots$ & $\cdots$ & $\ldots$ & $\ldots$ & $\ldots$ \\
\hline MACS J0417.5-1154 & $\cdots$ & $\cdots$ & $\ldots$ & $\cdots$ & $\cdots$ & $\cdots$ & $\cdots$ & $\cdots$ & $\cdots$ & $\ldots$ & $\ldots$ & $\ldots$ & $\ldots$ & $\ldots$ \\
\hline MACS J0429.6-0253 & $\cdots$ & $\cdots$ & $\cdots$ & $\cdots$ & $\cdots$ & $\cdots$ & $\cdots$ & $\cdots$ & $\cdots$ & $\cdots$ & $\cdots$ & $\cdots$ & $\cdots$ & $\cdots$ \\
\hline MACS J0520.7-1328 & $\ldots$ & $\ldots$ & $\ldots$ & $\ldots$ & $\ldots$ & $\ldots$ & $\ldots$ & $\ldots$ & $\ldots$ & $\ldots$ & $\ldots$ & $\ldots$ & $\ldots$ & $\ldots$ \\
\hline MACS J0547.0-3904 & $\cdots$ & $\cdots$ & $\cdots$ & $\cdots$ & $\cdots$ & $\cdots$ & $\cdots$ & $\cdots$ & $\cdots$ & $\cdots$ & $\cdots$ & $\cdots$ & $\cdots$ & $\cdots$ \\
\hline MACS J0717.5+3745 & $\cdots$ & $\cdots$ & $\cdots$ & $\cdots$ & $\cdots$ & $\cdots$ & $\cdots$ & $\cdots$ & $\cdots$ & $\cdots$ & $\cdots$ & $\cdots$ & $\cdots$ & \\
\hline MACS J0744.8+3927 & $\cdots$ & $\cdots$ & $\cdots$ & $\cdots$ & $\cdots$ & $\cdots$ & $\cdots$ & $\cdots$ & $\cdots$ & $\cdots$ & $\cdots$ & $\cdots$ & $\cdots$ & $\cdots$ \\
\hline MACS J1115.2+5320 & $\ldots$ & $\ldots$ & $\ldots$ & $\ldots$ & $\ldots$ & $\ldots$ & $\ldots$ & $\ldots$ & $\cdots$ & $\ldots$ & $\ldots$ & $\ldots$ & $\ldots$ & $\ldots$ \\
\hline MACS J1115.8+0129 & $\cdots$ & $\cdots$ & $\cdots$ & $\cdots$ & $\cdots$ & $\cdots$ & $\cdots$ & $\cdots$ & $\cdots$ & $\cdots$ & $\ldots$ & $\ldots$ & $\ldots$ & $\cdots$ \\
\hline MACS J1131.8-1955 & $\cdots$ & $\cdots$ & $\ldots$ & $\cdots$ & $\cdots$ & $\cdots$ & $\ldots$ & $\cdots$ & $\cdots$ & $\cdots$ & $\cdots$ & $\ldots$ & $\ldots$ & $\cdots$ \\
\hline MACS J1149.5+2223 & $\cdots$ & $\cdots$ & $\cdots$ & $\cdots$ & $\cdots$ & $\cdots$ & $\cdots$ & $\cdots$ & $\cdots$ & $\cdots$ & $\cdots$ & $\cdots$ & $\cdots$ & \\
\hline MACS J1206.2-0847 & $\cdots$ & $\cdots$ & $\cdots$ & $\cdots$ & $\cdots$ & $\cdots$ & $\cdots$ & $\cdots$ & $\cdots$ & $\cdots$ & $\cdots$ & $\cdots$ & $\cdots$ & $\ldots$ \\
\hline
\end{tabular}


Table 5-Continued

\begin{tabular}{|c|c|c|c|c|c|c|c|c|c|c|c|c|c|c|}
\hline $\begin{array}{c}\text { Name } \\
\text { Cluster Name }\end{array}$ & $\begin{array}{c}\text { Flux } \\
3.6 \mu m \\
(\mathrm{mJy})\end{array}$ & $\begin{array}{c}\text { Error } \\
3.6 \mu m \\
(\mathrm{mJy})\end{array}$ & $\begin{array}{c}\text { Flux } \\
4.5 \mu m \\
(\mathrm{mJy})\end{array}$ & $\begin{array}{l}\text { Error } \\
4.5 \mu m \\
(\mathrm{mJy})\end{array}$ & $\begin{array}{c}\text { Flux } \\
5.8 \mu m \\
(\mathrm{mJy})\end{array}$ & $\begin{array}{r}\text { Error } \\
5.8 \mu m \\
(\mathrm{mJy})\end{array}$ & $\begin{array}{c}\text { Flux } \\
8.0 \mu m \\
(\mathrm{mJy})\end{array}$ & $\begin{array}{c}\text { Error } \\
8.0 \mu m \\
(\mathrm{mJy})\end{array}$ & $\begin{array}{c}\text { Flux } \\
24 \mu m \\
(\mathrm{mJy})\end{array}$ & $\begin{array}{c}\text { Error } \\
24 \mu m \\
(\mathrm{mJy})\end{array}$ & $\begin{array}{l}\text { Flux } \\
70 \mu m \\
\text { (mJy) }\end{array}$ & $\begin{array}{c}\text { Error } \\
70 \mu m \\
(\mathrm{mJy})\end{array}$ & $\begin{array}{l}\text { Flux } \\
160 \mu m \\
\text { (mJy) }\end{array}$ & $\begin{array}{c}\text { Error } \\
160 \mu m \\
(\mathrm{mJy})\end{array}$ \\
\hline MACS J1311.0-0310 & $\cdots$ & $\cdots$ & $\ldots$ & $\cdots$ & $\cdots$ & $\cdots$ & $\cdots$ & $\ldots$ & $\cdots$ & $\cdots$ & $\ldots$ & $\ldots$ & $\ldots$ & $\cdots$ \\
\hline MACS J1621.3+3810 & $\cdots$ & $\cdots$ & $\cdots$ & $\cdots$ & $\cdots$ & $\cdots$ & $\cdots$ & $\cdots$ & $\cdots$ & $\cdots$ & $\cdots$ & $\ldots$ & $\ldots$ & $\cdots$ \\
\hline MACS J1931.8-2634 & $\ldots$ & $\cdots$ & $\cdots$ & $\cdots$ & $\ldots$ & $\ldots$ & $\cdots$ & $\cdots$ & $\ldots$ & $\cdots$ & $\ldots$ & $\ldots$ & $\ldots$ & 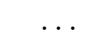 \\
\hline MACS J2049.9-3217 & $\cdots$ & $\cdots$ & $\cdots$ & $\cdots$ & $\cdots$ & $\cdots$ & $\cdots$ & $\cdots$ & $\ldots$ & $\cdots$ & $\cdots$ & $\ldots$ & $\cdots$ & $\cdots$ \\
\hline MACS J2211.7-0349 & $\cdots$ & $\cdots$ & $\cdots$ & $\cdots$ & $\cdots$ & $\cdots$ & $\ldots$ & $\cdots$ & 0.22 & 0.14 & $\ldots$ & $\ldots$ & $\cdots$ & $\ldots$ \\
\hline MACS J2214.9-1359 & $\ldots$ & $\ldots$ & $\ldots$ & $\ldots$ & $\ldots$ & $\ldots$ & $\ldots$ & $\ldots$ & $\ldots$ & $\ldots$ & $\ldots$ & $\ldots$ & $\ldots$ & $\cdots$ \\
\hline MACS J2228+2036 & $\ldots$ & $\ldots$ & $\ldots$ & $\ldots$ & $\ldots$ & $\ldots$ & $\ldots$ & $\ldots$ & $\ldots$ & $\ldots$ & $\ldots$ & $\ldots$ & $\ldots$ & $\ldots$ \\
\hline MACS J2229.7-2755 & $\ldots$ & $\ldots$ & $\ldots$ & $\cdots$ & $\ldots$ & $\ldots$ & $\ldots$ & $\ldots$ & $\ldots$ & $\ldots$ & $\ldots$ & $\ldots$ & $\ldots$ & $\ldots$ \\
\hline MACS J2245.0+2637 & $\ldots$ & $\ldots$ & $\cdots$ & $\cdots$ & $\ldots$ & $\ldots$ & $\ldots$ & $\cdots$ & $\ldots$ & $\ldots$ & $\ldots$ & $\ldots$ & $\ldots$ & .. \\
\hline MKW3S & $\ldots$ & $\ldots$ & $\ldots$ & $\ldots$ & $\ldots$ & $\ldots$ & $\ldots$ & $\ldots$ & $\ldots$ & $\ldots$ & $\ldots$ & $\ldots$ & $\ldots$ & $\ldots$ \\
\hline $\mathrm{MKW} 04^{\mathrm{e}}$ & 87.04 & 4.35 & 51.15 & 2.56 & 39.97 & 2.00 & 24.80 & 1.24 & 3.57 & 0.37 & 7.29 & 0 & 23.43 & 0 \\
\hline MKW 08 & $\ldots$ & $\ldots$ & $\ldots$ & $\ldots$ & $\ldots$ & $\ldots$ & $\ldots$ & $\ldots$ & $\ldots$ & $\ldots$ & $\ldots$ & $\ldots$ & $\ldots$ & $\cdots$ \\
\hline MS 0016.9+1609 & $\cdots$ & $\cdots$ & $\cdots$ & $\cdots$ & $\cdots$ & $\cdots$ & $\cdots$ & $\cdots$ & $\cdots$ & $\cdots$ & $\cdots$ & $\ldots$ & $\cdots$ & $\cdots$ \\
\hline MS 0116.3-0115 & $\ldots$ & $\ldots$ & $\cdots$ & $\cdots$ & $\cdots$ & $\ldots$ & $\ldots$ & $\cdots$ & $\cdots$ & $\ldots$ & $\cdots$ & $\ldots$ & $\cdots$ & $\cdots$ \\
\hline MS 0440.5+0204 & $\ldots$ & $\ldots$ & $\ldots$ & $\ldots$ & $\cdots$ & $\cdots$ & $\ldots$ & $\ldots$ & $\ldots$ & $\ldots$ & $\cdots$ & $\ldots$ & $\ldots$ & $\cdots$ \\
\hline MS 0451.6-0305 & 0.36 & 0.02 & 0.25 & 0.01 & 0.18 & 0.01 & 0.11 & 0.01 & $0.15^{\mathrm{b}}$ & 0.02 & $2.23^{\mathrm{a}}$ & 0 & $2.68^{\mathrm{a}}$ & 0 \\
\hline MS $0735.6+7421$ & 0.92 & 0.05 & 0.71 & 0.04 & 0.44 & 0.02 & 0.33 & 0.02 & $\ldots$ & $\ldots$ & $\ldots$ & $\ldots$ & $\ldots$ & $\cdots$ \\
\hline MS 0839.8+2938 & $\ldots$ & $\ldots$ & $\ldots$ & $\ldots$ & $\ldots$ & $\ldots$ & $\ldots$ & $\ldots$ & 0.35 & 0.05 & $\ldots$ & $\ldots$ & $\ldots$ & $\ldots$ \\
\hline MS 0906.5+1110 & $\cdots$ & $\cdots$ & $\cdots$ & $\cdots$ & $\cdots$ & $\cdots$ & $\cdots$ & $\cdots$ & 0.48 & 0 & $\cdots$ & $\cdots$ & $\cdots$ & $\cdots$ \\
\hline MS $1006.0+1202$ & $\ldots$ & $\ldots$ & $\ldots$ & $\ldots$ & $\ldots$ & $\ldots$ & $\ldots$ & $\ldots$ & $\ldots$ & $\ldots$ & $\ldots$ & $\ldots$ & $\ldots$ & $\cdots$ \\
\hline MS 1008.1-1224 & 0.78 & 0.04 & 0.62 & 0.03 & 0.38 & 0.02 & 0.26 & 0.01 & $\ldots$ & $\ldots$ & $\ldots$ & $\ldots$ & $\ldots$ & $\cdots$ \\
\hline MS $1455.0+2232$ & 1.13 & 0.06 & 0.91 & 0.05 & 0.55 & 0.03 & 0.45 & 0.02 & 0.62 & 0.09 & $\ldots$ & $\ldots$ & $\ldots$ & $\cdots$ \\
\hline MS 2137.3-2353 & 0.81 & 0.04 & 0.64 & 0.03 & 0.44 & 0.02 & 0.27 & 0.01 & 0.58 & 0.13 & $\cdots$ & $\ldots$ & $\cdots$ & $\cdots$ \\
\hline MS J1157.3+5531 & $\ldots$ & $\ldots$ & $\ldots$ & $\ldots$ & $\ldots$ & $\ldots$ & $\ldots$ & $\ldots$ & $\ldots$ & $\ldots$ & $\ldots$ & $\ldots$ & $\ldots$ & $\ldots$ \\
\hline NGC $0507^{\mathrm{e}}$ & 98.66 & 4.93 & 59.01 & 2.95 & 49.18 & 2.46 & 28.78 & 1.44 & $8.27^{\mathrm{f}}$ & 0.83 & 4.15 & 0 & 2.19 & 0 \\
\hline NGC 4636 & 992.05 & 49.60 & 634.90 & 31.74 & 371.46 & 18.57 & 277.01 & $13.85^{\mathrm{f}}$ & 22.56 & 2.26 & 168.85 & 43.86 & 181.33 & 48.45 \\
\hline NGC 5044 & 244.53 & 12.23 & 143.78 & 7.19 & 121.75 & 6.09 & 76.38 & 3.82 & $32.24^{\mathrm{f}}$ & 3.23 & 140.06 & 29.34 & 251.76 & 50.82 \\
\hline NGC $5813^{\mathrm{e}}$ & 323.11 & 16.16 & 189.30 & 9.47 & 167.55 & 8.38 & 98.08 & 4.90 & $29.22^{\mathrm{f}}$ & f 2.93 & 76.25 & 29.14 & 137.18 & 59.69 \\
\hline NGC $5846^{\mathrm{e}}$ & 534.30 & 26.72 & 310.56 & 15.53 & 282.50 & 14.13 & 162.40 & 8.12 & $57.03^{\mathrm{f}}$ & f 5.71 & 219.11 & 50.86 & 287.15 & 71.78 \\
\hline
\end{tabular}


Table 5-Continued

\begin{tabular}{|c|c|c|c|c|c|c|c|c|c|c|c|c|c|c|}
\hline $\begin{array}{c}\text { Name } \\
\text { Cluster Name }\end{array}$ & $\begin{array}{c}\text { Flux } \\
3.6 \mu m \\
\text { (mJy) }\end{array}$ & $\begin{array}{l}\text { Error } \\
3.6 \mu m \\
(\mathrm{mJy})\end{array}$ & $\begin{array}{c}\text { Flux } \\
4.5 \mu m \\
(\mathrm{mJy})\end{array}$ & $\begin{array}{c}\text { Error } \\
4.5 \mu m \\
(\mathrm{mJy})\end{array}$ & $\begin{array}{c}\text { Flux } \\
5.8 \mu m \\
(\mathrm{mJy})\end{array}$ & $\begin{array}{r}\text { Error } \\
5.8 \mu m \\
(\mathrm{mJy})\end{array}$ & $\begin{array}{c}\text { Flux } \\
8.0 \mu m \\
(\mathrm{mJy})\end{array}$ & $\begin{array}{l}\text { Error } \\
8.0 \mu m \\
(\mathrm{mJy})\end{array}$ & $\begin{array}{c}\text { Flux } \\
24 \mu m \\
(\mathrm{mJy})\end{array}$ & $\begin{array}{c}\text { Error } \\
24 \mu m \\
(\mathrm{mJy})\end{array}$ & $\begin{array}{l}\text { Flux } \\
70 \mu m \\
\text { (mJy) }\end{array}$ & $\begin{array}{c}\text { Error } \\
70 \mu m \\
(\mathrm{mJy})\end{array}$ & $\begin{array}{l}\text { Flux } \\
160 \mu m \\
\text { (mJy) }\end{array}$ & $\begin{array}{c}\text { Error } \\
160 \mu m \\
(\mathrm{mJy})\end{array}$ \\
\hline OPHIUCHUS & $\cdots$ & $\cdots$ & $\cdots$ & $\cdots$ & $\cdots$ & $\cdots$ & $\cdots$ & $\cdots$ & $\ldots$ & $\cdots$ & $\ldots$ & $\ldots$ & $\ldots$ & $\ldots$ \\
\hline PKS 0745-191 & 4.51 & 0.23 & 3.16 & 0.16 & 2.31 & 0.12 & 2.93 & 0.15 & $10.22^{\mathrm{b}}$ & 1.02 & 154.33 & 34.50 & $\ldots$ & $\cdots$ \\
\hline RBS 0461 & $\ldots$ & $\ldots$ & $\ldots$ & $\ldots$ & $\ldots$ & $\ldots$ & $\ldots$ & $\ldots$ & $\ldots$ & $\ldots$ & $\ldots$ & $\ldots$ & $\ldots$ & $\ldots$ \\
\hline RBS 0533 & $\cdots$ & $\cdots$ & $\cdots$ & $\cdots$ & $\cdots$ & $\cdots$ & $\cdots$ & $\cdots$ & $\cdots$ & $\cdots$ & $\cdots$ & $\ldots$ & $\cdots$ & $\ldots$ \\
\hline RBS 0797 & $\cdots$ & $\ldots$ & $\cdots$ & $\ldots$ & $\cdots$ & $\cdots$ & $\cdots$ & $\cdots$ & $\ldots$ & $\cdots$ & $\cdots$ & $\cdots$ & $\ldots$ & $\ldots$ \\
\hline RCS J2327-0204 & $\cdots$ & $\cdots$ & $\cdots$ & $\cdots$ & $\cdots$ & $\cdots$ & $\cdots$ & $\cdots$ & $\cdots$ & $\cdots$ & $\cdots$ & $\cdots$ & $\cdots$ & $\cdots$ \\
\hline RXCJ0331.1-2100 & $\cdots$ & $\cdots$ & $\cdots$ & $\cdots$ & $\cdots$ & $\cdots$ & $\cdots$ & $\cdots$ & 2.28 & 0.23 & $\cdots$ & $\cdots$ & $\ldots$ & $\cdots$ \\
\hline RXC J1023.8-2715 & $\ldots$ & $\ldots$ & $\ldots$ & $\ldots$ & $\ldots$ & $\ldots$ & $\ldots$ & $\ldots$ & $\ldots$ & $\ldots$ & $\ldots$ & $\ldots$ & $\ldots$ & $\ldots$ \\
\hline RX J0220.9-3829 & $\cdots$ & $\cdots$ & $\cdots$ & $\cdots$ & $\cdots$ & $\cdots$ & $\cdots$ & $\cdots$ & $\cdots$ & $\cdots$ & $\cdots$ & $\cdots$ & $\cdots$ & $\cdots$ \\
\hline RX J0232.2-4420 & $\ldots$ & $\ldots$ & $\ldots$ & $\ldots$ & $\ldots$ & $\ldots$ & $\ldots$ & $\ldots$ & $\ldots$ & $\ldots$ & $\ldots$ & $\ldots$ & $\ldots$ & $\ldots$ \\
\hline RX J0439+0520 & 1.35 & 0.07 & 1.08 & 0.05 & 0.67 & 0.03 & 0.73 & 0.04 & 2.05 & 0.23 & $14.14^{\mathrm{d}}$ & d 2.97 & $\ldots$ & $\ldots$ \\
\hline RX J0439.0+0715 & $\cdots$ & $\cdots$ & $\ldots$ & $\cdots$ & $\cdots$ & $\ldots$ & $\cdots$ & $\ldots$ & $\cdots$ & $\cdots$ & $\cdots$ & $\cdots$ & $\cdots$ & $\cdots$ \\
\hline RX J0528.9-3927 & $\cdots$ & $\cdots$ & $\cdots$ & $\cdots$ & $\cdots$ & $\cdots$ & $\cdots$ & $\cdots$ & $\cdots$ & $\cdots$ & $\cdots$ & $\cdots$ & $\ldots$ & $\cdots$ \\
\hline RX J0647.7+7015 & $\cdots$ & $\cdots$ & $\cdots$ & $\cdots$ & $\cdots$ & $\cdots$ & $\cdots$ & $\cdots$ & $\cdots$ & $\cdots$ & $\cdots$ & $\cdots$ & $\cdots$ & $\cdots$ \\
\hline RX J0819.6+6336 & $\cdots$ & $\cdots$ & $\ldots$ & $\ldots$ & $\cdots$ & $\cdots$ & $\cdots$ & $\ldots$ & $\cdots$ & $\cdots$ & $\cdots$ & $\cdots$ & $\cdots$ & $\ldots$ \\
\hline RX J1000.4+4409 & $\cdots$ & $\cdots$ & $\cdots$ & $\cdots$ & $\cdots$ & $\cdots$ & $\cdots$ & $\cdots$ & $\cdots$ & $\cdots$ & $\cdots$ & $\cdots$ & $\cdots$ & $\cdots$ \\
\hline RX J1022.1+3830 & $\cdots$ & $\ldots$ & $\cdots$ & $\ldots$ & $\cdots$ & $\ldots$ & $\cdots$ & $\cdots$ & $\cdots$ & $\ldots$ & $\cdots$ & $\ldots$ & $\ldots$ & $\ldots$ \\
\hline RX J1130.0+3637 & $\cdots$ & $\cdots$ & $\cdots$ & $\cdots$ & $\cdots$ & $\cdots$ & $\cdots$ & $\cdots$ & $\cdots$ & $\cdots$ & $\cdots$ & $\cdots$ & $\cdots$ & $\cdots$ \\
\hline RX J1320.2+3308 & $\ldots$ & $\cdots$ & $\ldots$ & $\cdots$ & $\cdots$ & $\cdots$ & $\cdots$ & $\cdots$ & $\cdots$ & $\cdots$ & $\cdots$ & $\cdots$ & $\cdots$ & $\cdots$ \\
\hline RX J1347.5-1145 & 0.23 & 0.01 & 0.17 & 0.01 & 0.13 & 0.01 & 0.09 & 0 & $\cdots$ & $\ldots$ & $\cdots$ & $\ldots$ & $\ldots$ & $\ldots$ \\
\hline RX J1423.8+2404 & $\ldots$ & $\ldots$ & $\ldots$ & $\ldots$ & $\cdots$ & $\ldots$ & $\cdots$ & $\cdots$ & $\ldots$ & $\cdots$ & $\cdots$ & $\cdots$ & $\cdots$ & $\cdots$ \\
\hline RX J1504.1-0248 & $\ldots$ & $\cdots$ & $\ldots$ & $\cdots$ & $\cdots$ & $\ldots$ & $\cdots$ & $\ldots$ & 1.50 & 0.15 & $\ldots$ & $\ldots$ & $\ldots$ & $\ldots$ \\
\hline RX J1532.9+3021 & 0.69 & 0.03 & 0.61 & 0.03 & 0.39 & 0.02 & 0.80 & 0.04 & $3.76^{\mathrm{c}}$ & 0.38 & 90.71 & 18.14 & $\cdots$ & $\cdots$ \\
\hline RX J1539.5-8335 & $\cdots$ & $\cdots$ & $\cdots$ & $\cdots$ & $\cdots$ & $\cdots$ & $\cdots$ & $\cdots$ & $\cdots$ & $\cdots$ & $\ldots$ & $\ldots$ & $\cdots$ & $\cdots$ \\
\hline RX J1720.1+2638 & $\cdots$ & $\ldots$ & $\cdots$ & $\cdots$ & $\cdots$ & $\cdots$ & $\cdots$ & $\cdots$ & 0.55 & 0.08 & 6.92 & 0 & $\ldots$ & $\cdots$ \\
\hline RX J1720.2+3536 & $\cdots$ & $\cdots$ & $\cdots$ & $\cdots$ & $\cdots$ & $\cdots$ & $\cdots$ & $\cdots$ & $\cdots$ & $\cdots$ & $\cdots$ & $\cdots$ & $\cdots$ & $\cdots$ \\
\hline RX J1852.1+5711 & $\ldots$ & $\cdots$ & $\ldots$ & $\cdots$ & $\cdots$ & $\ldots$ & $\ldots$ & $\ldots$ & $\ldots$ & $\cdots$ & $\cdots$ & $\cdots$ & $\ldots$ & $\ldots$ \\
\hline RX J2129.6+0005 & 1.10 & 0.05 & 0.87 & 0.04 & 0.50 & 0.02 & 0.39 & 0.02 & 1.13 & 0.11 & 6.18 & 0 & $\cdots$ & $\cdots$ \\
\hline SC 1327-312 & $\ldots$ & $\ldots$ & $\ldots$ & $\ldots$ & $\cdots$ & $\ldots$ & $\ldots$ & $\ldots$ & $\ldots$ & $\ldots$ & $\ldots$ & $\cdots$ & $\ldots$ & $\ldots$ \\
\hline
\end{tabular}


Table 5-Continued

\begin{tabular}{|c|c|c|c|c|c|c|c|c|c|c|c|c|c|c|}
\hline $\begin{array}{c}\text { Name } \\
\text { Cluster Name }\end{array}$ & $\begin{array}{c}\text { Flux } \\
3.6 \mu m \\
\text { (mJy) }\end{array}$ & $\begin{array}{c}\text { Error } \\
3.6 \mu m \\
(\mathrm{mJy})\end{array}$ & $\begin{array}{c}\text { Flux } \\
4.5 \mu m \\
(\mathrm{mJy})\end{array}$ & $\begin{array}{r}\text { Error } \\
4.5 \mu m \\
(\mathrm{mJy})\end{array}$ & $\begin{array}{c}\text { Flux } \\
5.8 \mu m \\
\text { (mJy) }\end{array}$ & $\begin{array}{l}\text { Error } \\
5.8 \mu \mathrm{m} \\
\text { (mJy) }\end{array}$ & $\begin{array}{c}\text { Flux } \\
8.0 \mu m \\
(\mathrm{mJy})\end{array}$ & $\begin{array}{r}\text { Error } \\
8.0 \mu m \\
(\mathrm{mJy})\end{array}$ & $\begin{array}{l}\text { Flux } \\
24 \mu m \\
\text { (mJy) }\end{array}$ & $\begin{array}{l}\text { Error } \\
24 \mu m \\
(\mathrm{mJy})\end{array}$ & $\begin{array}{c}\text { Flux } \\
70 \mu m \\
(\mathrm{mJy})\end{array}$ & $\begin{array}{l}\text { Error } \\
70 \mu m \\
(\mathrm{mJy})\end{array}$ & $\begin{array}{l}\text { Flux } \\
160 \mu m 1 \\
(\mathrm{mJy})\end{array}$ & $\begin{array}{l}\text { Error } \\
160 \mu m \\
(\mathrm{mJy})\end{array}$ \\
\hline SERSIC 159-03 & 8.69 & 0.43 & 5.45 & 0.27 & 3.85 & 0.19 & 2.75 & 0.14 & 1.22 & 0.13 & $15.51^{\mathrm{d}}$ & 3.43 & $\ldots$ & \\
\hline SS2B153 & $\cdots$ & $\ldots$ & $\cdots$ & $\cdots$ & $\cdots$ & $\cdots$ & $\cdots$ & $\cdots$ & $\cdots$ & $\cdots$ & $\ldots$ & $\ldots$ & $\cdots$ & $\cdots$ \\
\hline UGC 03957 & $\cdots$ & $\cdots$ & $\cdots$ & $\cdots$ & $\cdots$ & $\cdots$ & $\cdots$ & $\cdots$ & $\cdots$ & $\cdots$ & $\cdots$ & $\cdots$ & $\cdots$ & $\cdots$ \\
\hline UGC 12491 & $\ldots$ & $\ldots$ & $\ldots$ & $\ldots$ & $\ldots$ & $\ldots$ & $\ldots$ & $\ldots$ & $\ldots$ & $\ldots$ & $\ldots$ & $\ldots$ & $\ldots$ & $\ldots$ \\
\hline ZWCL 1215 & $\cdots$ & $\cdots$ & $\ldots$ & $\cdots$ & $\ldots$ & $\ldots$ & $\cdots$ & $\ldots$ & $\cdots$ & $\ldots$ & $\cdots$ & $\cdots$ & $\ldots$ & $\cdots$ \\
\hline ZWCL 1358+6245 & 0.38 & 0.02 & 0.30 & 0.02 & 0.19 & 0.01 & 0.12 & 0.01 & 0.19 & 0.03 & $\cdots$ & $\cdots$ & $\cdots$ & $\cdots$ \\
\hline ZWCL 1742 & 4.66 & 0.23 & 3.09 & 0.15 & 2.25 & 0.11 & 2.14 & 0.11 & $3.23^{\mathrm{b}}$ & 0.32 & $27.36^{\mathrm{d}}$ & 5.80 & $\cdots$ & \\
\hline ZWCL 1953 & $\cdots$ & $\cdots$ & $\cdots$ & $\cdots$ & $\ldots$ & $\cdots$ & $\cdots$ & $\cdots$ & $\cdots$ & $\cdots$ & $\ldots$ & $\ldots$ & $\cdots$ & $\cdots$ \\
\hline ZWCL 3146 & 0.84 & 0.04 & 0.76 & 0.04 & 0.51 & 0.03 & 1.20 & 0.06 & $4.35^{\mathrm{c}}$ & 0.44 & 46.42 & 14.05 & 156.84 & 38.26 \\
\hline ZWICKY 2701 & 1.02 & 0.05 & 0.76 & 0.04 & 0.46 & 0.02 & 0.31 & 0.02 & $0.20^{\mathrm{b}}$ & 0.05 & 6.67 & 0 & $\ldots$ & \\
\hline $\mathrm{ZwCl} 0857.9+2107$ & 0.82 & 0.04 & 1.01 & 0.05 & 1.58 & 0.08 & 3.56 & 0.18 & 33.03 & 3.30 & 214.18 & 42.84 & $\ldots$ & \\
\hline
\end{tabular}

a Originally, aperture flux measurement indicated a detection. However, 24 micron flux measurement and visual inspection indicated contamination in the aperture where flux is likely from an unrelated source. The reported measurement is now an upper limit computed using the point source estimate at $16^{\prime \prime}$.

${ }^{\mathrm{b}}$ Source not extended but has significant contamination. Point source measurement at $35^{\prime \prime}$ radius is greater than 10 percent error margin.

"Source not extended but has mild source contamination. Point source measurement at $35^{\prime \prime}$ radius is within 10 percent error.

${ }^{\mathrm{d}}$ Flux measurement is a filtered detection.

${ }^{\mathrm{e}}$ IRAC flux measurements derived from a BCD image that was remosaicked.

${ }^{\mathrm{f}}$ Source is extended in the MIPS 24 micron image. 24 micron flux measured within the aperture (see Table 3 ). Note an aperture radius of $35^{\prime \prime}$ was used for NGC4636 because of significant point source contamination outside of 
Note. - Fluxes reported with errors equal to 0 are $5 \sigma$ upper limits. 
Table 6. 2MASS Aperture Flux.

\begin{tabular}{|c|c|c|c|c|c|c|c|c|}
\hline $\begin{array}{c}\text { Name } \\
\text { Cluster Name }\end{array}$ & $\begin{array}{c}\text { Flux } \\
\text { J } \\
(\mathrm{mJy})\end{array}$ & $\begin{array}{c}\text { Error } \\
\mathrm{J} \\
(\mathrm{mJy})\end{array}$ & $\begin{array}{c}\text { Flux } \\
\text { H } \\
(\mathrm{mJy})\end{array}$ & $\begin{array}{c}\text { Error } \\
\text { H } \\
(\mathrm{mJy})\end{array}$ & $\begin{array}{c}\text { Flux } \\
\text { K } \\
\text { (mJy) }\end{array}$ & $\begin{array}{c}\text { Error } \\
\text { K } \\
\text { (mJy) }\end{array}$ & $\begin{array}{c}\text { Flux } \\
\text { K24 } \\
\text { (mJy) }\end{array}$ & $\begin{array}{c}\text { Error } \\
\text { K24 } \\
(\mathrm{mJy})\end{array}$ \\
\hline $1 \mathrm{E} 065756 \mathrm{a}$ & $\cdots$ & $\cdots$ & $\cdots$ & $\cdots$ & $\cdots$ & $\cdots$ & $\cdots$ & $\cdots$ \\
\hline 1E0657 56b & $\ldots$ & $\ldots$ & $\ldots$ & $\ldots$ & $\ldots$ & $\ldots$ & $\ldots$ & $\ldots$ \\
\hline 2A $0335+096$ & 65.69 & 0.54 & 72.49 & 0.94 & 57.94 & 0.83 & 40.23 & 0.50 \\
\hline 2PIGG J0011.5-2850 & 12.83 & 0.22 & 15.56 & 0.34 & 14.53 & 0.41 & $\ldots$ & $\ldots$ \\
\hline 2PIGG J2227.0-3041 & 15.75 & 0.26 & 19.44 & 0.37 & 17.22 & 0.41 & $\cdots$ & $\cdots$ \\
\hline $3 \mathrm{C} 28.0$ & 1.26 & 0.12 & 1.64 & 0.17 & 1.68 & 0.19 & 3.35 & 0.51 \\
\hline $3 \mathrm{C} 295$ & 0.76 & 0.11 & 1.03 & 0.18 & 1.31 & 0.17 & $\ldots$ & $\cdots$ \\
\hline $3 \mathrm{C} 388$ & 10.31 & 0.18 & 12.48 & 0.28 & 11.57 & 0.32 & 15.38 & 0.49 \\
\hline $4 \mathrm{C} 55.16$ & 0.63 & 0.12 & 0.97 & 0.20 & 1.07 & 0.19 & $\ldots$ & $\cdots$ \\
\hline Abell 13 & 5.56 & 0.19 & 7.24 & 0.31 & 6.79 & 0.32 & $\ldots$ & $\ldots$ \\
\hline Abell 68 & 1.52 & 0.13 & 1.55 & 0.21 & 2.04 & 0.23 & 4.47 & 0.60 \\
\hline Abell 85 & 27.30 & 0.28 & 34.94 & 0.43 & 30.85 & 0.57 & 30.31 & 0.56 \\
\hline Abell 119 & 35.72 & 0.36 & 44.95 & 0.62 & 39.83 & 0.70 & $\ldots$ & $\ldots$ \\
\hline Abell 133 & 22.75 & 0.38 & 27.42 & 0.71 & 25.98 & 0.63 & $\cdots$ & $\ldots$ \\
\hline Abell 141 & 0.88 & 0.11 & 1.32 & 0.17 & 1.22 & 0.19 & $\ldots$ & $\ldots$ \\
\hline Abell 160 & 25.68 & 0.43 & 30.24 & 0.58 & 27.38 & 0.85 & $\cdots$ & $\cdots$ \\
\hline Abell 193 & 37.18 & 0.35 & 47.36 & 0.50 & 39.79 & 0.60 & $\ldots$ & $\ldots$ \\
\hline Abell 209 & 2.01 & 0.12 & 2.27 & 0.19 & 2.67 & 0.20 & 5.94 & 0.53 \\
\hline Abell 222 & 1.43 & 0.11 & 1.75 & 0.17 & 2.05 & 0.20 & $\ldots$ & $\ldots$ \\
\hline Abell 223 & 0.95 & 0.11 & 1.42 & 0.18 & 1.78 & 0.20 & $\ldots$ & $\ldots$ \\
\hline Abell 262 & 175.12 & 1.44 & 234.38 & 2.23 & 176.68 & 1.78 & 73.44 & 0.44 \\
\hline Abell 267 & 1.85 & 0.12 & 2.11 & 0.20 & 2.78 & 0.19 & 4.99 & 0.49 \\
\hline Abell 368 & $\ldots$ & $\ldots$ & $\ldots$ & $\ldots$ & $\ldots$ & $\ldots$ & $\ldots$ & $\ldots$ \\
\hline Abell 370 & 0.79 & 0.11 & 0.99 & 0.15 & 1.56 & 0.20 & 2.44 & 0.54 \\
\hline Abell 383 & 2.60 & 0.13 & 2.92 & 0.20 & 3.37 & 0.22 & 6.26 & 0.59 \\
\hline Abell 399 & 15.64 & 0.25 & 18.06 & 0.42 & 16.71 & 0.43 & $\ldots$ & $\ldots$ \\
\hline Abell 400 & 126.33 & 0.86 & 148.84 & 1.47 & 122.78 & 1.30 & $\ldots$ & $\ldots$ \\
\hline Abell 401 & 16.14 & 0.24 & 20.03 & 0.38 & 17.75 & 0.40 & 22.10 & 0.53 \\
\hline Abell 426 & 297.55 & 7.75 & 338.24 & 10.76 & 279.04 & 10.73 & 110.50 & 3.74 \\
\hline Abell 478 & 13.93 & 0.17 & 15.61 & 0.28 & 13.36 & 0.24 & 17.60 & 0.37 \\
\hline Abell 496 & 74.64 & 0.52 & 87.26 & 0.88 & 70.20 & 0.99 & $\ldots$ & $\ldots$ \\
\hline Abell 520 & 1.66 & 0.17 & 1.75 & 0.24 & 1.82 & 0.27 & $\ldots$ & $\ldots$ \\
\hline Abell 521 & 1.40 & 0.11 & 1.90 & 0.16 & 1.86 & 0.22 & 3.77 & 0.57 \\
\hline Abell 539 & 68.99 & 0.68 & 80.66 & 1.13 & 65.27 & 1.08 & $\ldots$ & $\ldots$ \\
\hline Abell 562 & 4.93 & 0.18 & 6.17 & 0.29 & 5.88 & 0.29 & $\ldots$ & $\ldots$ \\
\hline Abell 576 & 34.31 & 0.45 & 39.45 & 0.71 & 33.51 & 0.74 & $\cdots$ & $\ldots$ \\
\hline Abell 586 & 2.72 & 0.12 & 3.08 & 0.19 & 3.75 & 0.21 & 8.14 & 0.54 \\
\hline Abell 611 & 1.09 & 0.13 & 1.83 & 0.20 & 1.85 & 0.18 & 3.82 & 0.48 \\
\hline Abell 644 & 12.69 & 0.27 & 14.88 & 0.41 & 12.44 & 0.52 & $\ldots$ & $\ldots$ \\
\hline Abell 665 & 2.03 & 0.12 & 2.69 & 0.20 & 2.46 & 0.19 & 3.71 & 0.50 \\
\hline Abell 697 & 1.54 & 0.13 & 2.03 & 0.20 & 2.64 & 0.20 & 4.63 & 0.54 \\
\hline Abell 744 & 11.34 & 0.26 & 14.31 & 0.44 & 13.17 & 0.36 & $\cdots$ & $\ldots$ \\
\hline Abell 754 & 17.31 & 0.33 & 21.07 & 0.42 & 17.09 & 0.65 & $\ldots$ & $\ldots$ \\
\hline Abell 773 & 1.83 & 0.13 & 2.12 & 0.17 & 2.57 & 0.19 & 4.65 & 0.49 \\
\hline
\end{tabular}


Table 6-Continued

\begin{tabular}{|c|c|c|c|c|c|c|c|c|}
\hline $\begin{array}{c}\text { Name } \\
\text { Cluster Name }\end{array}$ & $\begin{array}{c}\text { Flux } \\
\text { J } \\
(\mathrm{mJy})\end{array}$ & $\begin{array}{c}\text { Error } \\
\text { J } \\
(\mathrm{mJy})\end{array}$ & $\begin{array}{c}\text { Flux } \\
\text { H } \\
\text { (mJy) }\end{array}$ & $\begin{array}{c}\text { Error } \\
\mathrm{H} \\
(\mathrm{mJy})\end{array}$ & $\begin{array}{c}\text { Flux } \\
\text { K } \\
(\mathrm{mJy})\end{array}$ & $\begin{array}{c}\text { Error } \\
\mathrm{K} \\
(\mathrm{mJy})\end{array}$ & $\begin{array}{c}\text { Flux } \\
\text { K24 } \\
(\mathrm{mJy})\end{array}$ & $\begin{array}{c}\text { Error } \\
\text { K24 } \\
(\mathrm{mJy})\end{array}$ \\
\hline Abell 907 & 2.60 & 0.12 & 3.41 & 0.17 & 3.93 & 0.25 & $\ldots$ & $\ldots$ \\
\hline Abell 963 & 2.20 & 0.12 & 2.97 & 0.18 & 3.52 & 0.18 & $\ldots$ & $\cdots$ \\
\hline Abell 1060 & 284.71 & 12.60 & 277.40 & 13.40 & 246.57 & 17.61 & $\ldots$ & $\ldots$ \\
\hline Abell 1063S & 1.15 & 0.15 & 1.99 & 0.22 & 1.84 & 0.25 & 4.09 & 0.66 \\
\hline Abell 1068 & 3.94 & 0.13 & 5.43 & 0.21 & 5.61 & 0.22 & 9.72 & 0.48 \\
\hline Abell 1201 & 2.30 & 0.10 & 2.75 & 0.17 & 3.38 & 0.18 & $\ldots$ & $\ldots$ \\
\hline Abell 1204 & 1.70 & 0.10 & 1.88 & 0.16 & 2.08 & 0.17 & 3.50 & 0.46 \\
\hline Abell 1240 & $\ldots$ & $\ldots$ & $\ldots$ & $\ldots$ & $\ldots$ & $\ldots$ & $\ldots$ & $\ldots$ \\
\hline Abell 1361 & 4.17 & 0.14 & 4.97 & 0.24 & 5.27 & 0.26 & 9.36 & 0.52 \\
\hline Abell 1413 & 4.80 & 0.12 & 6.13 & 0.19 & 5.75 & 0.21 & $\ldots$ & $\ldots$ \\
\hline Abell 1423 & 1.60 & 0.10 & 2.21 & 0.14 & 2.60 & 0.17 & $\ldots$ & $\ldots$ \\
\hline Abell 1446 & 6.18 & 0.17 & 6.99 & 0.28 & 7.15 & 0.27 & $\ldots$ & $\ldots$ \\
\hline Abell 1569 & 8.63 & 0.21 & 10.76 & 0.36 & 9.55 & 0.34 & $\cdots$ & $\cdots$ \\
\hline Abell 1576 & 1.34 & 0.11 & 1.49 & 0.18 & 2.37 & 0.18 & $\ldots$ & $\ldots$ \\
\hline Abell 1644 & 34.13 & 0.45 & 42.28 & 0.62 & 36.09 & 0.76 & $\cdots$ & $\cdots$ \\
\hline Abell 1650 & 8.54 & 0.23 & 10.86 & 0.36 & 9.06 & 0.47 & $\ldots$ & $\ldots$ \\
\hline Abell 1651 & 9.68 & 0.23 & 13.02 & 0.32 & 11.96 & 0.42 & $\ldots$ & $\ldots$ \\
\hline Abell 1664 & 3.64 & 0.15 & 4.50 & 0.26 & 4.32 & 0.26 & 6.52 & 0.54 \\
\hline Abell 1689 & 2.19 & 0.12 & 2.45 & 0.18 & 3.04 & 0.24 & 6.21 & 0.64 \\
\hline Abell 1736 & 35.70 & 0.53 & 42.11 & 0.72 & 37.68 & 1.02 & $\ldots$ & $\ldots$ \\
\hline Abell 1758 & 0.94 & 0.11 & 1.42 & 0.18 & 1.46 & 0.18 & 2.70 & 0.55 \\
\hline Abell 1763 & 0.64 & 0.08 & 0.75 & 0.13 & 1.23 & 0.15 & 2.63 & 0.39 \\
\hline Abell 1795 & 13.86 & 0.24 & 17.85 & 0.33 & 15.35 & 0.39 & 16.48 & 0.42 \\
\hline Abell 1835 & 2.41 & 0.14 & 2.81 & 0.20 & 3.59 & 0.26 & 5.37 & 0.69 \\
\hline Abell 1914 & 1.48 & 0.09 & 1.97 & 0.13 & 1.78 & 0.18 & 2.61 & 0.55 \\
\hline Abell 1942 & 1.89 & 0.14 & 2.04 & 0.19 & 2.87 & 0.24 & $\ldots$ & $\ldots$ \\
\hline Abell 1991 & 16.35 & 0.30 & 20.46 & 0.40 & 17.32 & 0.55 & $\ldots$ & $\ldots$ \\
\hline Abell 1995 & 0.74 & 0.10 & 0.89 & 0.14 & 1.50 & 0.15 & $\ldots$ & $\ldots$ \\
\hline Abell 2029 & 20.59 & 0.25 & 26.01 & 0.32 & 25.02 & 0.52 & 31.75 & 0.70 \\
\hline Abell 2034 & 5.30 & 0.15 & 6.67 & 0.23 & 6.57 & 0.26 & $\ldots$ & $\ldots$ \\
\hline Abell 2052 & 51.77 & 0.59 & 64.49 & 0.86 & 54.11 & 1.03 & 35.87 & 0.60 \\
\hline Abell 2063 & 40.82 & 0.54 & 48.63 & 0.83 & 40.55 & 0.99 & 27.79 & 0.61 \\
\hline Abell 2065 & 8.38 & 0.25 & 9.89 & 0.45 & 9.47 & 0.37 & $\ldots$ & $\ldots$ \\
\hline Abell 2069 & 4.50 & 0.15 & 6.00 & 0.26 & 5.47 & 0.25 & $\ldots$ & $\ldots$ \\
\hline Abell 2104 & 2.57 & 0.14 & 3.12 & 0.19 & 2.83 & 0.24 & $\ldots$ & $\ldots$ \\
\hline Abell 2107 & 45.92 & 0.41 & 56.67 & 0.56 & 48.82 & 0.80 & 39.08 & 0.57 \\
\hline Abell 2111 & 1.04 & 0.10 & 1.45 & 0.18 & 1.43 & 0.17 & $\ldots$ & $\ldots$ \\
\hline Abell 2124 & 15.29 & 0.25 & 18.53 & 0.37 & 17.63 & 0.36 & $\ldots$ & $\ldots$ \\
\hline Abell 2125 & 1.69 & 0.13 & 2.33 & 0.19 & 2.44 & 0.23 & 5.07 & 0.61 \\
\hline Abell 2142 & 7.03 & 0.21 & 8.82 & 0.29 & 7.40 & 0.38 & 10.61 & 0.58 \\
\hline Abell 2147 & 41.21 & 0.47 & 49.30 & 0.66 & 42.60 & 0.77 & $\ldots$ & $\ldots$ \\
\hline Abell 2151 & 45.08 & 0.43 & 53.44 & 0.70 & 46.29 & 0.70 & 31.18 & 0.46 \\
\hline Abell 2163 & $\ldots$ & $\ldots$ & $\ldots$ & $\ldots$ & $\ldots$ & $\ldots$ & $\ldots$ & $\ldots$ \\
\hline Abell 2187 & 2.38 & 0.11 & 3.14 & 0.15 & 3.39 & 0.19 & $\ldots$ & $\ldots$ \\
\hline
\end{tabular}


Table 6-Continued

\begin{tabular}{|c|c|c|c|c|c|c|c|c|}
\hline $\begin{array}{c}\text { Name } \\
\text { Cluster Name }\end{array}$ & $\begin{array}{c}\text { Flux } \\
\text { J } \\
(\mathrm{mJy})\end{array}$ & $\begin{array}{c}\text { Error } \\
\mathrm{J} \\
(\mathrm{mJy})\end{array}$ & $\begin{array}{c}\text { Flux } \\
\text { H } \\
(\mathrm{mJy})\end{array}$ & $\begin{array}{c}\text { Error } \\
\mathrm{H} \\
(\mathrm{mJy})\end{array}$ & $\begin{array}{c}\text { Flux } \\
\text { K } \\
(\mathrm{mJy})\end{array}$ & $\begin{array}{c}\text { Error } \\
\mathrm{K} \\
(\mathrm{mJy})\end{array}$ & $\begin{array}{c}\text { Flux } \\
\text { K24 } \\
(\mathrm{mJy})\end{array}$ & $\begin{array}{c}\text { Error } \\
\text { K24 } \\
(\mathrm{mJy})\end{array}$ \\
\hline Abell 2199 & 93.82 & 0.67 & 115.01 & 1.13 & 96.73 & 1.20 & 61.03 & 0.60 \\
\hline Abell 2204 & 3.27 & 0.11 & 3.40 & 0.17 & 3.37 & 0.21 & 11.15 & 0.52 \\
\hline Abell 2218 & $\ldots$ & $\ldots$ & $\ldots$ & $\ldots$ & $\ldots$ & $\ldots$ & $\ldots$ & $\ldots$ \\
\hline Abell 2219 & 1.26 & 0.12 & 1.95 & 0.19 & 1.92 & 0.21 & 4.73 & 0.56 \\
\hline Abell 2244 & 8.13 & 0.16 & 10.44 & 0.25 & 9.74 & 0.27 & $\ldots$ & $\ldots$ \\
\hline Abell 2255 & 4.17 & 0.23 & 5.27 & 0.39 & 4.72 & 0.41 & 5.71 & 0.57 \\
\hline Abell 2256 & 21.01 & 0.38 & 26.30 & 0.55 & 22.92 & 0.59 & $\ldots$ & $\ldots$ \\
\hline Abell 2259 & 2.75 & 0.12 & 3.51 & 0.16 & 3.86 & 0.19 & $\ldots$ & $\ldots$ \\
\hline Abell 2261 & 2.85 & 0.11 & 3.68 & 0.18 & 4.17 & 0.19 & 7.34 & 0.49 \\
\hline Abell 2294 & 3.62 & 0.13 & 4.31 & 0.19 & 4.95 & 0.23 & $\ldots$ & $\ldots$ \\
\hline Abell 2319 & 24.58 & 0.32 & 29.88 & 0.49 & 24.34 & 0.51 & $\ldots$ & $\ldots$ \\
\hline Abell 2384 & 4.82 & 0.18 & 5.40 & 0.31 & 5.61 & 0.34 & $\ldots$ & $\ldots$ \\
\hline Abell 2390 & 1.43 & 0.11 & 1.81 & 0.17 & 2.38 & 0.21 & 4.74 & 0.55 \\
\hline Abell 2409 & 2.68 & 0.16 & 3.04 & 0.23 & 3.80 & 0.27 & $\ldots$ & $\ldots$ \\
\hline Abell 2420 & 11.49 & 0.26 & 13.90 & 0.40 & 12.64 & 0.43 & $\ldots$ & $\ldots$ \\
\hline Abell 2462 & 12.91 & 0.27 & 15.73 & 0.47 & 14.29 & 0.46 & $\ldots$ & $\ldots$ \\
\hline Abell 2537 & 1.26 & 0.12 & 1.49 & 0.21 & 1.45 & 0.21 & 3.44 & 0.55 \\
\hline Abell 2554 & 5.39 & 0.17 & 6.07 & 0.32 & 6.03 & 0.31 & $\ldots$ & $\ldots$ \\
\hline Abell 2556 & 7.71 & 0.22 & 9.29 & 0.42 & 8.83 & 0.39 & $\ldots$ & $\ldots$ \\
\hline Abell 2589 & 36.96 & 0.45 & 46.09 & 0.61 & 38.49 & 0.88 & $\ldots$ & $\ldots$ \\
\hline Abell 2597 & 6.54 & 0.25 & 7.35 & 0.45 & 7.02 & 0.42 & 8.87 & 0.61 \\
\hline Abell 2626 & 21.15 & 0.32 & 25.97 & 0.52 & 22.67 & 0.58 & 22.86 & 0.59 \\
\hline Abell 2631 & 0.96 & 0.11 & 1.49 & 0.17 & 1.63 & 0.18 & $\ldots$ & $\ldots$ \\
\hline Abell 2657 & 24.29 & 0.51 & 27.73 & 0.78 & 22.12 & 0.83 & $\ldots$ & $\ldots$ \\
\hline Abell 2667 & 1.09 & 0.13 & 1.08 & 0.22 & 1.25 & 0.23 & 4.26 & 0.61 \\
\hline Abell 2717 & 20.25 & 0.35 & 25.45 & 0.49 & 23.37 & 0.73 & $\ldots$ & $\ldots$ \\
\hline Abell 2744a & $\ldots$ & $\ldots$ & $\ldots$ & $\ldots$ & $\ldots$ & $\ldots$ & $\cdots$ & $\cdots$ \\
\hline Abell 2744b & $\ldots$ & $\ldots$ & $\ldots$ & $\ldots$ & $\ldots$ & $\ldots$ & $\ldots$ & $\ldots$ \\
\hline Abell 2813 & 0.55 & 0.11 & 0.59 & 0.17 & 1.14 & 0.18 & $\ldots$ & $\cdots$ \\
\hline Abell 3084 & 3.56 & 0.21 & 4.89 & 0.31 & 5.58 & 0.35 & 7.66 & 0.58 \\
\hline Abell 3088 & 0.94 & 0.12 & 1.09 & 0.20 & 1.37 & 0.24 & 3.82 & 0.65 \\
\hline Abell 3112 & 13.58 & 0.25 & 16.17 & 0.31 & 15.59 & 0.39 & 18.76 & 0.49 \\
\hline Abell 3120 & 10.59 & 0.27 & 12.67 & 0.36 & 11.72 & 0.44 & $\ldots$ & $\ldots$ \\
\hline Abell 3158 & 18.32 & 0.35 & 24.32 & 0.56 & 20.55 & 0.58 & 20.78 & 0.60 \\
\hline Abell 3266 & 27.98 & 0.28 & 35.52 & 0.44 & 31.49 & 0.59 & 32.32 & 0.61 \\
\hline Abell 3364 & 2.57 & 0.16 & 3.09 & 0.22 & 3.01 & 0.29 & 5.86 & 0.70 \\
\hline Abell 3376 & 17.49 & 0.50 & 21.86 & 0.73 & 19.39 & 0.84 & $\ldots$ & $\cdots$ \\
\hline Abell 3391 & 37.75 & 0.36 & 46.94 & 0.54 & 39.17 & 0.61 & 38.88 & 0.60 \\
\hline Abell 3395 & 23.90 & 0.40 & 29.92 & 0.59 & 24.56 & 0.62 & $\ldots$ & $\ldots$ \\
\hline Abell 3528S & 51.40 & 0.40 & 63.21 & 0.57 & 54.18 & 0.73 & $\cdots$ & $\cdots$ \\
\hline Abell 3558 & 42.86 & 0.37 & 53.17 & 0.47 & 44.86 & 0.76 & $\ldots$ & $\ldots$ \\
\hline Abell 3562 & 26.50 & 0.42 & 32.02 & 0.55 & 27.91 & 0.72 & $\cdots$ & $\cdots$ \\
\hline Abell 3571 & 56.48 & 0.57 & 67.55 & 0.94 & 56.35 & 0.84 & $\cdots$ & $\cdots$ \\
\hline Abell 3581 & 91.59 & 0.97 & 111.17 & 1.12 & 93.04 & 1.65 & $\ldots$ & $\ldots$ \\
\hline
\end{tabular}


Table 6-Continued

\begin{tabular}{|c|c|c|c|c|c|c|c|c|}
\hline $\begin{array}{c}\text { Name } \\
\text { Cluster Name }\end{array}$ & $\begin{array}{c}\text { Flux } \\
\text { J } \\
(\mathrm{mJy})\end{array}$ & $\begin{array}{c}\text { Error } \\
\mathrm{J} \\
(\mathrm{mJy})\end{array}$ & $\begin{array}{c}\text { Flux } \\
\text { H } \\
(\mathrm{mJy})\end{array}$ & $\begin{array}{c}\text { Error } \\
\text { H } \\
(\mathrm{mJy})\end{array}$ & $\begin{array}{c}\text { Flux } \\
\text { K } \\
(\mathrm{mJy})\end{array}$ & $\begin{array}{c}\text { Error } \\
\mathrm{K} \\
(\mathrm{mJy})\end{array}$ & $\begin{array}{c}\text { Flux } \\
\text { K24 } \\
\text { (mJy) }\end{array}$ & $\begin{array}{c}\text { Error } \\
\text { K24 } \\
(\mathrm{mJy})\end{array}$ \\
\hline Abell 3667 & 26.10 & 0.38 & 32.59 & 0.51 & 27.58 & 0.62 & $\ldots$ & $\ldots$ \\
\hline Abell 3822 & 10.67 & 0.31 & 13.65 & 0.43 & 12.44 & 0.44 & $\ldots$ & $\ldots$ \\
\hline Abell 3827 & 11.98 & 0.24 & 15.22 & 0.35 & 13.46 & 0.38 & $\ldots$ & $\ldots$ \\
\hline Abell 3921 & 10.29 & 0.27 & 12.53 & 0.36 & 12.48 & 0.41 & $\ldots$ & $\ldots$ \\
\hline Abell 4038 & 52.87 & 0.74 & 66.47 & 1.25 & 53.26 & 1.20 & $\ldots$ & $\ldots$ \\
\hline Abell 4059 & 33.92 & 0.37 & 42.73 & 0.55 & 36.95 & 0.64 & 32.37 & 0.55 \\
\hline Abell S0405 & 20.37 & 0.38 & 25.16 & 0.54 & 22.12 & 0.65 & $\ldots$ & $\ldots$ \\
\hline Abell S0592 & 1.38 & 0.14 & 1.48 & 0.20 & 1.76 & 0.22 & 2.98 & 0.59 \\
\hline AC 114 & 1.04 & 0.12 & 1.62 & 0.17 & 1.56 & 0.23 & 3.61 & 0.61 \\
\hline AWM7 & 246.13 & 1.14 & 293.96 & 1.71 & 236.70 & 1.64 & $\ldots$ & $\ldots$ \\
\hline CENTAURUS & 846.43 & 9.05 & 784.14 & 11.56 & 671.20 & 10.08 & 147.71 & 2.69 \\
\hline CID 0072 & 35.35 & 0.51 & 44.73 & 0.78 & 36.12 & 0.90 & 27.69 & 0.54 \\
\hline CL J1226.9+3332 & $\ldots$ & $\ldots$ & $\ldots$ & $\ldots$ & $\ldots$ & $\ldots$ & $\ldots$ & $\ldots$ \\
\hline CYGNUS A & 36.44 & 0.43 & 41.18 & 0.55 & 34.72 & 0.60 & 34.47 & 0.60 \\
\hline ESO 3060170 & 48.06 & 0.63 & 58.38 & 0.83 & 49.06 & 1.02 & $\ldots$ & $\ldots$ \\
\hline ESO 5520200 & 73.87 & 0.67 & 88.69 & 0.95 & 74.30 & 1.21 & $\ldots$ & $\ldots$ \\
\hline EXO 0422-086 & 45.35 & 0.39 & 53.84 & 0.54 & 43.04 & 0.80 & $\ldots$ & $\ldots$ \\
\hline HCG 0062 & 232.69 & 1.68 & 280.96 & 2.58 & 217.50 & 3.30 & 91.57 & 0.70 \\
\hline HCG 42 & 350.28 & 1.90 & 423.08 & 3.13 & 343.69 & 3.63 & 163.65 & 0.67 \\
\hline HERCULES A & 2.03 & 0.13 & 2.43 & 0.19 & 3.10 & 0.21 & 5.92 & 0.51 \\
\hline HYDRA A & 21.02 & 0.46 & 25.11 & 0.70 & 22.79 & 0.70 & 22.41 & 0.68 \\
\hline M49 & 4144.27 & 180.56 & 4195.07 & 236.24 & 3698.43 & 275.45 & 561.11 & 11.22 \\
\hline M87 & 3303.44 & 104.40 & 3559.22 & 146.49 & 2882.03 & 130.89 & 523.58 & 10.45 \\
\hline MACS J0011.7-1523 & $\ldots$ & $\ldots$ & $\ldots$ & $\ldots$ & $\ldots$ & $\ldots$ & $\ldots$ & $\ldots$ \\
\hline MACS J0035.4-2015 & 0.69 & 0.09 & 0.95 & 0.15 & 1.21 & 0.19 & $\ldots$ & $\ldots$ \\
\hline MACS J0159.8-0849 & $\ldots$ & $\ldots$ & $\ldots$ & $\ldots$ & $\ldots$ & $\ldots$ & $\ldots$ & $\ldots$ \\
\hline MACS J0242.5-2132 & $\ldots$ & $\ldots$ & $\ldots$ & $\ldots$ & $\ldots$ & $\ldots$ & $\ldots$ & $\ldots$ \\
\hline MACS J0257.1-2325 & $\ldots$ & $\ldots$ & $\ldots$ & $\ldots$ & $\ldots$ & $\ldots$ & $\ldots$ & $\ldots$ \\
\hline MACS J0257.6-2209 & 1.17 & 0.13 & 1.54 & 0.22 & 2.10 & 0.22 & $\ldots$ & $\ldots$ \\
\hline MACS J0308.9+2645 & 1.69 & 0.10 & 2.06 & 0.16 & 2.36 & 0.17 & $\ldots$ & $\ldots$ \\
\hline MACS J0329.6-0211 & $\ldots$ & $\ldots$ & $\ldots$ & $\ldots$ & $\ldots$ & $\ldots$ & $\ldots$ & $\ldots$ \\
\hline MACS J0417.5-1154 & $\ldots$ & $\ldots$ & $\ldots$ & $\ldots$ & $\ldots$ & $\ldots$ & $\ldots$ & $\ldots$ \\
\hline MACS J0429.6-0253 & 0.71 & 0.11 & 1.20 & 0.19 & 1.63 & 0.21 & $\ldots$ & $\ldots$ \\
\hline MACS J0520.7-1328 & 0.90 & 0.14 & 1.48 & 0.18 & 1.90 & 0.25 & $\ldots$ & $\ldots$ \\
\hline MACS J0547.0-3904 & $\ldots$ & $\ldots$ & $\ldots$ & $\ldots$ & $\ldots$ & $\ldots$ & $\ldots$ & $\ldots$ \\
\hline MACS J0717.5+3745 & $\ldots$ & $\ldots$ & $\ldots$ & $\ldots$ & $\ldots$ & $\ldots$ & $\ldots$ & $\ldots$ \\
\hline MACS J0744.8+3927 & $\ldots$ & $\ldots$ & $\ldots$ & $\ldots$ & $\ldots$ & $\ldots$ & $\ldots$ & $\ldots$ \\
\hline MACS J1115.2+5320 & $\ldots$ & $\cdots$ & $\cdots$ & $\ldots$ & $\ldots$ & $\ldots$ & $\ldots$ & $\ldots$ \\
\hline MACS J1115.8+0129 & $\ldots$ & $\ldots$ & $\ldots$ & $\ldots$ & $\ldots$ & $\ldots$ & $\ldots$ & $\ldots$ \\
\hline MACS J1131.8-1955 & 1.27 & 0.11 & 1.77 & 0.19 & 2.26 & 0.22 & $\ldots$ & $\ldots$ \\
\hline MACS J1149.5+2223 & $\ldots$ & $\ldots$ & $\ldots$ & $\ldots$ & $\ldots$ & $\ldots$ & $\ldots$ & $\ldots$ \\
\hline MACS J1206.2-0847 & $\ldots$ & $\ldots$ & $\ldots$ & $\ldots$ & $\ldots$ & $\ldots$ & $\ldots$ & $\ldots$ \\
\hline MACS J1311.0-0310 & $\ldots$ & $\ldots$ & $\ldots$ & $\ldots$ & $\ldots$ & $\ldots$ & $\ldots$ & $\ldots$ \\
\hline MACS J1621.3+3810 & $\ldots$ & $\ldots$ & $\ldots$ & $\ldots$ & $\ldots$ & $\ldots$ & $\ldots$ & $\ldots$ \\
\hline
\end{tabular}


Table 6-Continued

\begin{tabular}{|c|c|c|c|c|c|c|c|c|}
\hline $\begin{array}{c}\text { Name } \\
\text { Cluster Name }\end{array}$ & $\begin{array}{c}\text { Flux } \\
\text { J } \\
(\mathrm{mJy})\end{array}$ & $\begin{array}{c}\text { Error } \\
\mathrm{J} \\
(\mathrm{mJy})\end{array}$ & $\begin{array}{c}\text { Flux } \\
\text { H } \\
(\mathrm{mJy})\end{array}$ & $\begin{array}{c}\text { Error } \\
\text { H } \\
(\mathrm{mJy})\end{array}$ & $\begin{array}{c}\text { Flux } \\
\text { K } \\
(\mathrm{mJy})\end{array}$ & $\begin{array}{c}\text { Error } \\
\mathrm{K} \\
(\mathrm{mJy})\end{array}$ & $\begin{array}{c}\text { Flux } \\
\text { K24 } \\
\text { (mJy) }\end{array}$ & $\begin{array}{c}\text { Error } \\
\text { K24 } \\
(\mathrm{mJy})\end{array}$ \\
\hline MACS J1931.8-2634 & $\cdots$ & $\ldots$ & $\ldots$ & $\ldots$ & $\ldots$ & $\ldots$ & $\ldots$ & $\cdots$ \\
\hline MACS J2049.9-3217 & $\cdots$ & $\cdots$ & $\cdots$ & $\cdots$ & $\cdots$ & $\ldots$ & $\cdots$ & $\cdots$ \\
\hline MACS J2211.7-0349 & 0.87 & 0.14 & 1.80 & 0.21 & 1.72 & 0.23 & 4.13 & 0.61 \\
\hline MACS J2214.9-1359 & $\cdots$ & $\cdots$ & $\cdots$ & $\cdots$ & $\cdots$ & $\cdots$ & $\cdots$ & $\cdots$ \\
\hline MACS J2228+2036 & $\ldots$ & $\ldots$ & $\ldots$ & $\ldots$ & $\ldots$ & $\ldots$ & $\ldots$ & $\ldots$ \\
\hline MACS J2229.7-2755 & 0.81 & 0.10 & 1.20 & 0.16 & 1.51 & 0.17 & $\ldots$ & $\ldots$ \\
\hline MACS J2245.0+2637 & 1.26 & 0.12 & 1.19 & 0.18 & 1.93 & 0.20 & $\ldots$ & $\cdots$ \\
\hline MKW3S & 23.64 & 0.40 & 29.06 & 0.56 & 24.13 & 0.73 & $\ldots$ & $\cdots$ \\
\hline MKW 04 & 196.90 & 1.23 & 242.96 & 1.64 & 198.43 & 2.16 & 97.48 & 0.67 \\
\hline MKW 08 & 47.78 & 0.90 & 56.21 & 1.29 & 52.13 & 1.46 & $\ldots$ & $\ldots$ \\
\hline MS 0016.9+1609 & $\ldots$ & $\ldots$ & $\ldots$ & $\cdots$ & $\ldots$ & $\ldots$ & $\cdots$ & $\cdots$ \\
\hline MS 0116.3-0115 & 31.25 & 0.39 & 37.88 & 0.63 & 31.35 & 0.64 & $\ldots$ & $\ldots$ \\
\hline MS $0440.5+0204$ & 5.85 & 0.19 & 6.85 & 0.25 & 6.86 & 0.27 & $\cdots$ & $\cdots$ \\
\hline MS 0451.6-0305 & $\ldots$ & $\ldots$ & $\ldots$ & $\ldots$ & $\ldots$ & $\ldots$ & $\ldots$ & $\ldots$ \\
\hline MS $0735.6+7421$ & 1.37 & 0.11 & 1.61 & 0.19 & 1.83 & 0.20 & $\cdots$ & $\cdots$ \\
\hline MS $0839.8+2938$ & 1.74 & 0.08 & 2.11 & 0.12 & 2.60 & 0.16 & 4.61 & 0.42 \\
\hline MS 0906.5+1110 & 2.86 & 0.15 & 3.19 & 0.21 & 3.73 & 0.26 & 7.08 & 0.68 \\
\hline MS $1006.0+1202$ & 1.36 & 0.15 & 1.86 & 0.24 & 2.22 & 0.27 & $\ldots$ & $\ldots$ \\
\hline MS 1008.1-1224 & 1.33 & 0.14 & 1.55 & 0.21 & 1.69 & 0.26 & $\ldots$ & $\ldots$ \\
\hline MS $1455.0+2232$ & 1.68 & 0.11 & 1.90 & 0.18 & 2.66 & 0.20 & 4.86 & 0.54 \\
\hline MS 2137.3-2353 & 1.09 & 0.09 & 1.22 & 0.13 & 1.68 & 0.16 & 2.58 & 0.42 \\
\hline MS J1157.3+5531 & 12.93 & 0.21 & 16.55 & 0.33 & 14.50 & 0.33 & $\ldots$ & $\ldots$ \\
\hline NGC 0507 & 256.60 & 1.42 & 285.59 & 2.38 & 247.06 & 2.11 & 117.08 & 0.52 \\
\hline NGC 4636 & 1948.19 & 87.98 & 2080.16 & 122.03 & 1687.71 & 135.74 & 263.45 & 7.02 \\
\hline NGC 5044 & 628.83 & 2.80 & 731.33 & 4.56 & 599.60 & 7.20 & 174.24 & 0.77 \\
\hline NGC 5813 & 891.14 & 35.99 & 867.64 & 38.30 & 733.94 & 64.40 & 183.53 & 8.86 \\
\hline NGC 5846 & 2430.17 & 52.65 & 2780.30 & 65.20 & 2181.73 & 16.18 & 267.15 & 0.71 \\
\hline OPHIUCHUS & 192.74 & 1.47 & 186.81 & 1.90 & 135.70 & 1.71 & $\ldots$ & $\ldots$ \\
\hline PKS 0745-191 & 13.46 & 0.21 & 13.88 & 0.26 & 10.93 & 0.38 & 15.13 & 0.67 \\
\hline RBS 0461 & 52.87 & 0.63 & 58.47 & 0.84 & 43.29 & 1.24 & $\ldots$ & $\ldots$ \\
\hline RBS 0533 & 242.51 & 2.55 & 285.11 & 3.23 & 225.51 & 4.08 & $\cdots$ & $\cdots$ \\
\hline RBS 0797 & $\ldots$ & $\ldots$ & $\ldots$ & $\ldots$ & $\ldots$ & $\ldots$ & $\ldots$ & $\ldots$ \\
\hline RCS J2327-0204 & $\cdots$ & $\cdots$ & $\cdots$ & $\cdots$ & $\cdots$ & $\cdots$ & $\cdots$ & $\cdots$ \\
\hline RXCJ0331.1-2100 & 5.82 & 0.12 & 6.60 & 0.18 & 5.43 & 0.22 & 7.98 & 0.59 \\
\hline RXC J1023.8-2715 & 2.07 & 0.20 & 1.94 & 0.24 & 2.39 & 0.36 & $\ldots$ & $\ldots$ \\
\hline RX J0220.9-3829 & 1.25 & 0.11 & 1.36 & 0.16 & 1.51 & 0.19 & $\ldots$ & $\ldots$ \\
\hline RX J0232.2-4420 & $\cdots$ & $\cdots$ & $\cdots$ & $\cdots$ & $\cdots$ & $\cdots$ & $\cdots$ & $\cdots$ \\
\hline RX J0439+0520 & 2.26 & 0.14 & 2.54 & 0.22 & 3.37 & 0.25 & 5.36 & 0.66 \\
\hline RX J0439.0+0715 & 0.95 & 0.12 & 1.61 & 0.20 & 1.77 & 0.18 & $\ldots$ & $\ldots$ \\
\hline RX J0528.9-3927 & $\cdots$ & $\cdots$ & $\cdots$ & $\cdots$ & $\cdots$ & $\cdots$ & $\cdots$ & $\cdots$ \\
\hline RX J0647.7+7015 & $\ldots$ & $\ldots$ & $\ldots$ & $\ldots$ & $\ldots$ & $\ldots$ & $\ldots$ & $\ldots$ \\
\hline RX J0819.6+6336 & 6.99 & 0.14 & 8.65 & 0.21 & 8.44 & 0.23 & $\cdots$ & $\cdots$ \\
\hline RX J1000.4+4409 & $\ldots$ & $\ldots$ & $\ldots$ & $\ldots$ & $\ldots$ & $\ldots$ & $\ldots$ & $\ldots$ \\
\hline RX J1022.1+3830 & 16.97 & 0.33 & 20.76 & 0.54 & 17.50 & 0.54 & $\cdots$ & $\cdots$ \\
\hline
\end{tabular}


Table 6-Continued

\begin{tabular}{|c|c|c|c|c|c|c|c|c|}
\hline $\begin{array}{c}\text { Name } \\
\text { Cluster Name }\end{array}$ & $\begin{array}{c}\text { Flux } \\
\text { J } \\
(\mathrm{mJy})\end{array}$ & $\begin{array}{c}\text { Error } \\
\mathrm{J} \\
(\mathrm{mJy})\end{array}$ & $\begin{array}{c}\text { Flux } \\
\text { H } \\
(\mathrm{mJy})\end{array}$ & $\begin{array}{c}\text { Error } \\
\mathrm{H} \\
(\mathrm{mJy})\end{array}$ & $\begin{array}{c}\text { Flux } \\
\text { K } \\
\text { (mJy) }\end{array}$ & $\begin{array}{c}\text { Error } \\
\text { K } \\
(\mathrm{mJy})\end{array}$ & $\begin{array}{l}\text { Flux } \\
\text { K24 } \\
\text { (mJy) }\end{array}$ & $\begin{array}{c}\text { Error } \\
\text { K24 } \\
(\mathrm{mJy})\end{array}$ \\
\hline RX J1130.0+3637 & 5.59 & 0.26 & 6.51 & 0.42 & 6.19 & 0.42 & $\cdots$ & $\cdots$ \\
\hline RX J1320.2+3308 & 25.95 & 0.41 & 32.15 & 0.55 & 27.38 & 0.71 & $\cdots$ & $\cdots$ \\
\hline RX J1347.5-1145 & $\ldots$ & $\ldots$ & $\ldots$ & $\ldots$ & $\ldots$ & $\ldots$ & $\ldots$ & $\ldots$ \\
\hline RX J1423.8+2404 & $\cdots$ & $\cdots$ & $\cdots$ & $\cdots$ & $\cdots$ & $\cdots$ & $\cdots$ & $\cdots$ \\
\hline RX J1504.1-0248 & 2.21 & 0.14 & 3.24 & 0.19 & 3.15 & 0.28 & 3.35 & 0.73 \\
\hline RX J1532.9+3021 & $\ldots$ & $\cdots$ & $\ldots$ & $\cdots$ & $\ldots$ & $\cdots$ & $\cdots$ & $\cdots$ \\
\hline RX J1539.5-8335 & 15.68 & 0.34 & 18.99 & 0.48 & 14.88 & 0.57 & $\ldots$ & $\ldots$ \\
\hline RX J1720.1+2638 & 2.77 & 0.12 & 3.47 & 0.16 & 3.54 & 0.19 & 7.01 & 0.49 \\
\hline RX J1720.2+3536 & $\cdots$ & $\cdots$ & $\cdots$ & $\cdots$ & $\cdots$ & $\cdots$ & $\cdots$ & $\cdots$ \\
\hline RX J1852.1+5711 & 3.26 & 0.16 & 3.99 & 0.22 & 3.92 & 0.31 & $\ldots$ & $\ldots$ \\
\hline RX J2129.6+0005 & 1.69 & 0.12 & 2.50 & 0.21 & 2.56 & 0.22 & 3.97 & 0.57 \\
\hline SC 1327-312 & 14.98 & 0.33 & 17.95 & 0.43 & 16.60 & 0.67 & $\ldots$ & $\cdots$ \\
\hline SERSIC 159-03 & 16.16 & 0.29 & 20.03 & 0.42 & 17.17 & 0.53 & 17.41 & 0.54 \\
\hline SS2B153 & 177.24 & 1.12 & 216.72 & 1.60 & 171.39 & 2.00 & $\ldots$ & $\ldots$ \\
\hline UGC 03957 & 65.94 & 0.53 & 80.20 & 0.86 & 67.14 & 0.80 & $\ldots$ & $\ldots$ \\
\hline UGC 12491 & 98.34 & 1.12 & 110.16 & 1.91 & 90.98 & 2.07 & $\ldots$ & $\ldots$ \\
\hline ZWCL 1215 & 9.87 & 0.27 & 12.11 & 0.38 & 11.57 & 0.52 & $\ldots$ & $\ldots$ \\
\hline ZWCL $1358+6245$ & $\ldots$ & $\ldots$ & $\ldots$ & $\ldots$ & $\ldots$ & $\ldots$ & $\ldots$ & $\ldots$ \\
\hline ZWCL 1742 & 8.36 & 0.20 & 9.68 & 0.34 & 9.41 & 0.32 & 11.54 & 0.42 \\
\hline ZWCL 1953 & 0.71 & 0.10 & 1.35 & 0.18 & 1.13 & 0.17 & $\ldots$ & $\cdots$ \\
\hline ZWCL 3146 & 1.05 & 0.14 & 1.78 & 0.21 & 1.51 & 0.25 & 3.84 & 0.66 \\
\hline ZWICKY 2701 & 1.47 & 0.13 & 1.64 & 0.23 & 2.22 & 0.21 & 3.90 & 0.56 \\
\hline $\mathrm{ZwCl} 0857.9+2107$ & 0.76 & 0.10 & 0.92 & 0.13 & 1.40 & 0.17 & 2.81 & 0.44 \\
\hline
\end{tabular}


Table 7. Star Formation Rates:

\begin{tabular}{|c|c|c|c|c|c|c|c|c|}
\hline $\begin{array}{c}\text { Name } \\
\text { Cluster Name }\end{array}$ & $\begin{array}{c}\mathrm{SFR} \\
\mathrm{UV} \\
\left(M_{\odot} y r^{-1}\right.\end{array}$ & $\begin{array}{c}\text { Error } \\
\mathrm{UV} \\
\left.M_{\odot} y r^{-1}\right)\end{array}$ & $\begin{array}{c}\mathrm{SFR} \\
\mathrm{IR} \\
\left(M_{\odot} y r^{-1}\right)\end{array}$ & $\begin{array}{c}\mathrm{SFR} \\
70 \mu m \\
\left(M_{\odot} y r^{-1}\right)\end{array}$ & $\begin{array}{c}\text { Error } \\
70 \mu m \\
\left(M_{\odot} y r^{-1}\right)\end{array}$ & $\begin{array}{c}\text { SFR } \\
24 \mu m \\
\left(M_{\odot} y r^{-1}\right)\end{array}$ & $\begin{array}{c}\text { Error } \\
24 \mu m \\
\left(M_{\odot} y r^{-1}\right)\end{array}$ & $\begin{array}{c}\text { Mass } \\
\text { Stellar Mass } \\
\left(10^{10} M_{\odot}\right)\end{array}$ \\
\hline $1 \mathrm{E} 065756 \mathrm{a}$ & $\cdots$ & $\cdots$ & 2.12 & 5.22 & 0 & 0.91 & 0.11 & 5.52 \\
\hline $1 \mathrm{E} 065756 \mathrm{~b}$ & $\cdots$ & $\cdots$ & 1.64 & 1.65 & 0 & 0.76 & 0.12 & 6.68 \\
\hline 2A $0335+096$ & $\cdots$ & $\cdots$ & 0.72 & 0.94 & 0.25 & 0.21 & 0.02 & 5.58 \\
\hline 2PIGG J0011.5-2850 & 0.92 & 0 & $\ldots$ & $\ldots$ & $\ldots$ & $\ldots$ & $\cdots$ & $\ldots$ \\
\hline 2PIGG J2227.0-3041 & 1.82 & 0.33 & $\ldots$ & $\cdots$ & $\cdots$ & $\cdots$ & $\cdots$ & $\cdots$ \\
\hline $3 \mathrm{C} 28.0$ & 2.30 & 0.37 & 13.51 & 2.15 & 0 & 2.05 & 0.29 & 4.40 \\
\hline $3 \mathrm{C} 295$ & $\cdots$ & $\ldots$ & $\ldots$ & $\ldots$ & $\cdots$ & $\ldots$ & $\ldots$ & $\ldots$ \\
\hline 3C 388 & 1.14 & 0 & 1.78 & 1.61 & 0.36 & 1.47 & 0.15 & 7.06 \\
\hline $4 \mathrm{C} 55.16$ & $\cdots$ & $\cdots$ & $\cdots$ & $\cdots$ & $\cdots$ & $\cdots$ & $\cdots$ & $\cdots$ \\
\hline Abell 13 & $\ldots$ & $\ldots$ & $\ldots$ & $\ldots$ & $\ldots$ & $\ldots$ & $\ldots$ & $\ldots$ \\
\hline Abell 68 & $\ldots$ & $\ldots$ & $\ldots$ & $\ldots$ & $\ldots$ & $\ldots$ & $\ldots$ & $\ldots$ \\
\hline Abell 85 & 0.90 & 0 & 0.61 & 0.70 & 0.16 & 0.36 & 0.04 & 6.72 \\
\hline Abell 119 & 0.57 & 0 & $\ldots$ & $\ldots$ & $\ldots$ & $\ldots$ & $\ldots$ & $\ldots$ \\
\hline Abell 133 & 0.69 & 0 & $\ldots$ & $\ldots$ & $\ldots$ & $\ldots$ & $\ldots$ & $\ldots$ \\
\hline Abell 141 & $\ldots$ & $\ldots$ & $\cdots$ & $\ldots$ & $\cdots$ & $\ldots$ & $\cdots$ & $\ldots$ \\
\hline Abell 160 & 0.40 & 0 & $\ldots$ & $\ldots$ & $\ldots$ & $\ldots$ & $\ldots$ & $\ldots$ \\
\hline Abell 193 & $\ldots$ & $\ldots$ & $\ldots$ & $\ldots$ & $\ldots$ & $\ldots$ & $\cdots$ & $\cdots$ \\
\hline Abell 209 & $\ldots$ & $\ldots$ & 2.04 & $\ldots$ & $\ldots$ & 0.91 & 0.12 & 7.91 \\
\hline Abell 222 & $\cdots$ & $\cdots$ & $\ldots$ & $\cdots$ & $\cdots$ & $\ldots$ & $\ldots$ & $\ldots$ \\
\hline Abell 223 & $\ldots$ & $\ldots$ & $\cdots$ & $\ldots$ & $\cdots$ & $\ldots$ & $\ldots$ & $\ldots$ \\
\hline Abell 262 & 0.16 & 0 & 0.12 & 0.39 & 0.10 & 0.08 & 0.01 & 3.50 \\
\hline Abell 267 & 2.86 & 0 & 5.01 & $\ldots$ & $\cdots$ & 0.69 & 0.27 & 9.84 \\
\hline Abell 368 & $\ldots$ & $\ldots$ & $\ldots$ & $\ldots$ & $\ldots$ & $\ldots$ & $\ldots$ & $\ldots$ \\
\hline Abell 370 & $\ldots$ & $\ldots$ & 5.96 & 2.86 & 0 & 1.19 & 0.47 & 14.39 \\
\hline Abell 383 & 2.40 & 0 & 5.58 & $\ldots$ & $\ldots$ & 1.65 & 0.18 & 8.03 \\
\hline Abell 399 & $\cdots$ & $\ldots$ & $\ldots$ & $\ldots$ & $\ldots$ & $\ldots$ & $\ldots$ & $\ldots$ \\
\hline Abell 400 & 0.32 & 0 & $\cdots$ & $\cdots$ & $\cdots$ & $\cdots$ & $\cdots$ & $\ldots$ \\
\hline Abell 401 & 1.10 & 0 & $\ldots$ & $\ldots$ & 0 & $\ldots$ & $\ldots$ & $\ldots$ \\
\hline Abell 426 & 7.11 & 0.10 & 34.46 & 12.16 & 2.43 & 69.99 & 7.00 & 6.66 \\
\hline
\end{tabular}


Table 7-Continued

\begin{tabular}{|c|c|c|c|c|c|c|c|c|}
\hline $\begin{array}{c}\text { Name } \\
\text { Cluster Name }\end{array}$ & $\begin{array}{c}\text { SFR } \\
\mathrm{UV} \\
\left(M_{\odot} y r^{-}\right.\end{array}$ & $\begin{array}{l}\text { Error } \\
\mathrm{UV} \\
\left.M_{\odot} y r^{-1}\right)\end{array}$ & $\begin{array}{c}\mathrm{SFR} \\
\mathrm{IR} \\
\left(M_{\odot} y r^{-1}\right)\end{array}$ & $\begin{array}{c}\mathrm{SFR} \\
70 \mu m \\
\left(M_{\odot} y r^{-1}\right)\end{array}$ & $\begin{array}{c}\text { Error } \\
70 \mu m \\
\left(M_{\odot} y r^{-1}\right)\end{array}$ & $\begin{array}{c}\mathrm{SFR} \\
24 \mu m \\
\left(M_{\odot} y r^{-1}\right)\end{array}$ & $\begin{array}{c}\text { Error } \\
24 \mu m \\
\left(M_{\odot} y r^{-1}\right)\end{array}$ & $\begin{array}{c}\text { Mass } \\
\text { Stellar Mass } \\
\left(10^{10} M_{\odot}\right)\end{array}$ \\
\hline Abell 478 & $\cdots$ & $\ldots$ & 2.39 & 2.15 & 0.44 & 0.98 & 0.10 & 7.84 \\
\hline Abell 496 & $\cdots$ & $\ldots$ & $\ldots$ & $\ldots$ & $\ldots$ & $\ldots$ & $\ldots$ & $\ldots$ \\
\hline Abell 520 & $\ldots$ & $\ldots$ & $\ldots$ & $\ldots$ & $\ldots$ & $\ldots$ & $\ldots$ & $\ldots$ \\
\hline Abell 521 & $\ldots$ & $\ldots$ & $\cdots$ & $\ldots$ & $\ldots$ & $\ldots$ & $\ldots$ & $\ldots$ \\
\hline Abell 539 & 0.15 & 0.04 & $\ldots$ & $\ldots$ & $\ldots$ & $\ldots$ & $\ldots$ & $\ldots$ \\
\hline Abell 562 & $\ldots$ & $\ldots$ & $\cdots$ & $\ldots$ & $\cdots$ & $\ldots$ & $\ldots$ & $\cdots$ \\
\hline Abell 576 & $\cdots$ & $\cdots$ & $\ldots$ & $\ldots$ & $\cdots$ & $\ldots$ & $\cdots$ & $\cdots$ \\
\hline Abell 586 & $\ldots$ & $\ldots$ & $\ldots$ & $\ldots$ & $\ldots$ & $\ldots$ & $\ldots$ & $\ldots$ \\
\hline Abell 611 & $\ldots$ & $\ldots$ & $\cdots$ & $\ldots$ & $\cdots$ & $\ldots$ & $\ldots$ & $\cdots$ \\
\hline Abell 644 & 0.33 & 0 & $\ldots$ & $\ldots$ & $\ldots$ & $\ldots$ & $\ldots$ & $\ldots$ \\
\hline Abell 665 & $\ldots$ & $\ldots$ & $\ldots$ & $\cdots$ & $\cdots$ & $\ldots$ & $\ldots$ & $\ldots$ \\
\hline Abell 697 & 4.17 & 0 & 13.33 & $\ldots$ & $\cdots$ & 1.58 & 0.16 & 13.70 \\
\hline Abell 744 & 0.65 & 0 & $\ldots$ & $\ldots$ & $\cdots$ & $\ldots$ & $\ldots$ & $\ldots$ \\
\hline Abell 754 & $\ldots$ & $\ldots$ & $\ldots$ & $\ldots$ & $\cdots$ & $\ldots$ & $\ldots$ & $\ldots$ \\
\hline Abell 773 & $\ldots$ & $\ldots$ & 3.64 & $\ldots$ & $\cdots$ & 1.25 & 0.16 & 8.17 \\
\hline Abell 907 & $\ldots$ & $\ldots$ & $\ldots$ & $\ldots$ & $\cdots$ & $\ldots$ & $\ldots$ & $\ldots$ \\
\hline Abell 963 & 2.70 & 0 & $\ldots$ & $\ldots$ & $\ldots$ & $\ldots$ & $\ldots$ & $\ldots$ \\
\hline Abell 1060 & 0.10 & 0 & $\cdots$ & $\cdots$ & $\cdots$ & $\cdots$ & $\cdots$ & $\ldots$ \\
\hline Abell 1063S & $\cdots$ & $\cdots$ & 0.40 & $\ldots$ & $\ldots$ & 0.82 & 0.13 & 17.78 \\
\hline Abell 1068 & 2.60 & 0.60 & 93.15 & 159.87 & 32.12 & 119.39 & 11.94 & 6.08 \\
\hline Abell 1201 & $\cdots$ & $\cdots$ & $\ldots$ & $\ldots$ & $\ldots$ & $\ldots$ & $\ldots$ & $\cdots$ \\
\hline Abell 1204 & 0.76 & 0 & 4.44 & 8.10 & 1.68 & 3.72 & 0.40 & 4.27 \\
\hline Abell 1240 & $\ldots$ & $\cdots$ & $\ldots$ & $\ldots$ & $\cdots$ & $\ldots$ & $\ldots$ & $\cdots$ \\
\hline Abell 1361 & $\cdots$ & $\cdots$ & 3.21 & 2.06 & 0.44 & 0.73 & 0.08 & 4.94 \\
\hline Abell 1413 & 2.32 & 0 & $\ldots$ & $\ldots$ & $\ldots$ & $\ldots$ & $\ldots$ & $\cdots$ \\
\hline Abell 1423 & $\cdots$ & $\ldots$ & $\cdots$ & $\cdots$ & $\cdots$ & $\ldots$ & $\cdots$ & $\cdots$ \\
\hline Abell 1446 & 0.76 & 0 & $\cdots$ & $\cdots$ & $\cdots$ & $\cdots$ & $\cdots$ & $\cdots$ \\
\hline Abell 1569 & 0.36 & 0 & $\cdots$ & $\cdots$ & $\cdots$ & $\cdots$ & $\cdots$ & $\cdots$ \\
\hline Abell 1576 & $\cdots$ & $\cdots$ & $\cdots$ & $\cdots$ & $\cdots$ & $\cdots$ & $\cdots$ & $\cdots$ \\
\hline
\end{tabular}


Table 7-Continued

\begin{tabular}{|c|c|c|c|c|c|c|c|c|}
\hline $\begin{array}{c}\text { Name } \\
\text { Cluster Name }\end{array}$ & $\begin{array}{c}\mathrm{SFR} \\
\mathrm{UV} \\
\left(M_{\odot} y r^{-1}\right.\end{array}$ & $\begin{array}{c}\text { Error } \\
\mathrm{UV} \\
\left.M_{\odot} y r^{-1}\right)\end{array}$ & $\begin{array}{c}\mathrm{SFR} \\
\mathrm{IR} \\
\left(M_{\odot} y r^{-1}\right)\end{array}$ & $\begin{array}{c}\text { SFR } \\
70 \mu m \\
\left(M_{\odot} y r^{-1}\right)\end{array}$ & $\begin{array}{c}\text { Error } \\
70 \mu m \\
\left(M_{\odot} y r^{-1}\right)\end{array}$ & $\begin{array}{c}\mathrm{SFR} \\
24 \mu m \\
\left(M_{\odot} y r^{-1}\right)\end{array}$ & $\begin{array}{c}\text { Error } \\
24 \mu m \\
\left(M_{\odot} y r^{-1}\right)\end{array}$ & $\begin{array}{c}\text { Mass } \\
\text { Stellar Mass } \\
\left(10^{10} M_{\odot}\right)\end{array}$ \\
\hline Abell 1644 & 0.63 & 0 & $\ldots$ & $\ldots$ & $\cdots$ & $\ldots$ & $\cdots$ & $\ldots$ \\
\hline Abell 1650 & $\ldots$ & $\cdots$ & $\cdots$ & $\ldots$ & $\cdots$ & $\ldots$ & $\cdots$ & $\cdots$ \\
\hline Abell 1651 & $\ldots$ & $\ldots$ & $\ldots$ & $\ldots$ & $\cdots$ & $\ldots$ & $\ldots$ & $\ldots$ \\
\hline Abell 1664 & 16.13 & 0.27 & 10.00 & 11.53 & 3.12 & 5.80 & 0.58 & 4.90 \\
\hline Abell 1689 & $\ldots$ & $\cdots$ & 10.88 & 0.82 & 0 & 2.17 & 0.22 & 6.27 \\
\hline Abell 1736 & 0.23 & 0 & $\ldots$ & $\ldots$ & $\ldots$ & $\ldots$ & $\ldots$ & $\ldots$ \\
\hline Abell 1758 & $\ldots$ & $\ldots$ & 3.28 & $\ldots$ & $\ldots$ & 0.93 & 0.13 & 8.51 \\
\hline Abell 1763 & 1.06 & 0 & 7.68 & 2.26 & 0 & 1.14 & 0.34 & 4.18 \\
\hline Abell 1795 & 2.39 & 0.20 & $\ldots$ & $\ldots$ & $\ldots$ & $\ldots$ & $\ldots$ & $\ldots$ \\
\hline Abell 1835 & 23.56 & 1.07 & 235.37 & 134.96 & 27.86 & 97.17 & 9.72 & 15.03 \\
\hline Abell 1914 & 0.58 & 0 & 0.35 & $\ldots$ & $\ldots$ & 0.31 & 0.04 & 3.89 \\
\hline Abell 1942 & $\ldots$ & $\ldots$ & $\ldots$ & $\ldots$ & $\ldots$ & $\ldots$ & $\ldots$ & $\ldots$ \\
\hline Abell 1991 & 0.56 & 0 & $\ldots$ & $\ldots$ & $\ldots$ & $\ldots$ & $\ldots$ & $\ldots$ \\
\hline Abell 1995 & 2.36 & 0 & $\cdots$ & $\ldots$ & $\cdots$ & $\cdots$ & $\ldots$ & $\ldots$ \\
\hline Abell 2029 & 1.72 & 0 & $\cdots$ & $\ldots$ & $\cdots$ & $\cdots$ & $\ldots$ & $\ldots$ \\
\hline Abell 2034 & $\ldots$ & $\ldots$ & $\ldots$ & $\ldots$ & $\ldots$ & $\ldots$ & $\ldots$ & $\ldots$ \\
\hline Abell 2052 & 0.41 & 0 & 0.34 & 0.54 & 0.11 & 0.47 & 0.05 & 4.89 \\
\hline Abell 2063 & 0.31 & 0 & $\ldots$ & $\cdots$ & $\ldots$ & $\ldots$ & $\ldots$ & $\ldots$ \\
\hline Abell 2065 & 0.35 & 0 & $\cdots$ & $\ldots$ & $\ldots$ & $\ldots$ & $\ldots$ & $\ldots$ \\
\hline Abell 2069 & $\cdots$ & $\cdots$ & $\cdots$ & $\cdots$ & $\ldots$ & $\ldots$ & $\cdots$ & $\ldots$ \\
\hline Abell 2104 & $\ldots$ & $\ldots$ & $\ldots$ & $\ldots$ & $\ldots$ & $\ldots$ & $\ldots$ & $\cdots$ \\
\hline Abell 2107 & 0.57 & 0 & $\ldots$ & $\ldots$ & $\ldots$ & $\ldots$ & $\ldots$ & $\cdots$ \\
\hline Abell 2111 & 2.17 & 0 & $\cdots$ & $\cdots$ & $\cdots$ & $\cdots$ & $\ldots$ & $\cdots$ \\
\hline Abell 2124 & 0.76 & 0 & $\ldots$ & $\cdots$ & $\cdots$ & $\cdots$ & $\ldots$ & $\cdots$ \\
\hline Abell 2125 & $\cdots$ & $\ldots$ & $\ldots$ & $\cdots$ & $\cdots$ & $\ldots$ & $\cdots$ & $\cdots$ \\
\hline Abell 2142 & 0.77 & 0 & $\ldots$ & $\ldots$ & $\cdots$ & $\ldots$ & $\ldots$ & $\ldots$ \\
\hline Abell 2147 & 0.35 & 0 & $\ldots$ & $\ldots$ & $\cdots$ & $\cdots$ & $\ldots$ & $\cdots$ \\
\hline Abell 2151 & 0.38 & 0 & $\ldots$ & $\ldots$ & $\ldots$ & $\ldots$ & $\cdots$ & $\cdots$ \\
\hline Abell 2163 & $\cdots$ & $\ldots$ & $\ldots$ & $\cdots$ & $\ldots$ & $\ldots$ & $\ldots$ & $\cdots$ \\
\hline
\end{tabular}


Table 7-Continued

\begin{tabular}{|c|c|c|c|c|c|c|c|c|}
\hline $\begin{array}{c}\text { Name } \\
\text { Cluster Name }\end{array}$ & $\begin{array}{c}\text { SFR } \\
\mathrm{UV} \\
\left(M_{\odot} y r^{-}\right.\end{array}$ & $\begin{array}{c}\text { Error } \\
\mathrm{UV} \\
M_{\odot} y r^{-}\end{array}$ & $\begin{array}{c}\mathrm{SFR} \\
\mathrm{IR} \\
\left(M_{\odot} y r^{-1}\right)\end{array}$ & $\begin{array}{c}\mathrm{SFR} \\
70 \mu m \\
\left(M_{\odot} y r^{-1}\right)\end{array}$ & $\begin{array}{c}\text { Error } \\
70 \mu m \\
\left(M_{\odot} y r^{-1}\right)\end{array}$ & $\begin{array}{c}\mathrm{SFR} \\
24 \mu m \\
\left(M_{\odot} y r^{-1}\right)\end{array}$ & $\begin{array}{c}\text { Error } \\
24 \mu m \\
\left(M_{\odot} y r^{-1}\right)\end{array}$ & $\begin{array}{c}\text { Mass } \\
\text { Stellar Mass } \\
\left(10^{10} M_{\odot}\right)\end{array}$ \\
\hline Abell 2187 & 1.87 & 0 & $\ldots$ & $\cdots$ & $\cdots$ & $\ldots$ & $\ldots$ & $\ldots$ \\
\hline Abell 2199 & 0.49 & 0 & 0.58 & $\ldots$ & $\cdots$ & 0.35 & 0.03 & 6.35 \\
\hline Abell 2204 & $\ldots$ & $\cdots$ & 6.06 & 10.27 & 2.14 & 4.55 & 0.46 & 5.68 \\
\hline Abell 2218 & $\cdots$ & $\ldots$ & $\ldots$ & $\ldots$ & $\ldots$ & $\ldots$ & $\ldots$ & $\ldots$ \\
\hline Abell 2219 & $\ldots$ & $\ldots$ & 1.17 & $\ldots$ & $\ldots$ & 1.23 & 0.14 & 6.85 \\
\hline Abell 2244 & $\ldots$ & $\ldots$ & $\ldots$ & $\ldots$ & $\ldots$ & $\ldots$ & $\ldots$ & $\ldots$ \\
\hline Abell 2255 & 0.28 & 0 & $\cdots$ & $\cdots$ & $\cdots$ & $\cdots$ & $\cdots$ & $\cdots$ \\
\hline Abell 2256 & 0.56 & 0 & $\ldots$ & $\ldots$ & $\ldots$ & $\ldots$ & $\ldots$ & $\ldots$ \\
\hline Abell 2259 & $\ldots$ & $\ldots$ & $\ldots$ & $\ldots$ & $\ldots$ & $\ldots$ & $\ldots$ & $\ldots$ \\
\hline Abell 2261 & $\cdots$ & $\ldots$ & 9.99 & 1.54 & 0 & 1.58 & 0.17 & 13.54 \\
\hline Abell 2294 & $\cdots$ & $\ldots$ & $\ldots$ & $\ldots$ & $\cdots$ & $\ldots$ & $\ldots$ & $\ldots$ \\
\hline Abell 2319 & $\ldots$ & $\ldots$ & $\ldots$ & $\ldots$ & $\ldots$ & $\ldots$ & $\ldots$ & $\ldots$ \\
\hline Abell 2384 & $\ldots$ & $\ldots$ & $\ldots$ & $\ldots$ & $\ldots$ & $\ldots$ & $\ldots$ & $\ldots$ \\
\hline Abell 2390 & 8.53 & 0.86 & 40.60 & 20.76 & 5.35 & 6.14 & 0.62 & 7.20 \\
\hline Abell 2409 & $\cdots$ & $\ldots$ & $\ldots$ & $\ldots$ & $\cdots$ & $\ldots$ & $\ldots$ & $\cdots$ \\
\hline Abell 2420 & $\ldots$ & $\cdots$ & $\cdots$ & $\cdots$ & $\cdots$ & $\cdots$ & $\cdots$ & $\cdots$ \\
\hline Abell 2462 & 0.64 & 0 & $\ldots$ & $\ldots$ & $\ldots$ & $\ldots$ & $\ldots$ & $\ldots$ \\
\hline Abell 2537 & $\ldots$ & $\ldots$ & $\ldots$ & $\ldots$ & $\ldots$ & $\ldots$ & $\ldots$ & $\ldots$ \\
\hline Abell 2554 & 0.82 & 0 & $\cdots$ & $\cdots$ & $\cdots$ & $\cdots$ & $\cdots$ & $\cdots$ \\
\hline Abell 2556 & 0.70 & 0 & $\ldots$ & $\ldots$ & $\ldots$ & $\ldots$ & $\ldots$ & $\ldots$ \\
\hline Abell 2589 & 0.47 & 0 & $\ldots$ & $\ldots$ & $\ldots$ & $\ldots$ & $\ldots$ & $\ldots$ \\
\hline Abell 2597 & 2.52 & 0.19 & 3.23 & 3.31 & 1.00 & 1.37 & 0.15 & 3.90 \\
\hline Abell 2626 & 0.67 & 0 & 0.92 & 0.34 & 0 & 0.32 & 0.03 & 5.34 \\
\hline Abell 2631 & $\cdots$ & $\cdots$ & $\cdots$ & $\cdots$ & $\cdots$ & $\ldots$ & $\ldots$ & $\cdots$ \\
\hline Abell 2657 & $\ldots$ & $\ldots$ & $\ldots$ & $\ldots$ & $\ldots$ & $\ldots$ & $\ldots$ & $\ldots$ \\
\hline Abell 2667 & 2.32 & 0.68 & 75.94 & $\ldots$ & $\cdots$ & 31.97 & 3.20 & 4.84 \\
\hline Abell 2717 & 0.41 & 0 & $\ldots$ & $\ldots$ & $\cdots$ & $\ldots$ & $\cdots$ & $\cdots$ \\
\hline Abell 2744a & $\ldots$ & $\ldots$ & 36.16 & 4.88 & 0 & 2.31 & 0.25 & 7.94 \\
\hline Abell 2744b & $\cdots$ & $\cdots$ & $\ldots$ & $\ldots$ & $\cdots$ & $\cdots$ & $\ldots$ & $\ldots$ \\
\hline
\end{tabular}


Table 7-Continued

\begin{tabular}{|c|c|c|c|c|c|c|c|c|}
\hline $\begin{array}{c}\text { Name } \\
\text { Cluster Name }\end{array}$ & $\begin{array}{c}\mathrm{SFR} \\
\mathrm{UV} \\
\left(M_{\odot} y r^{-1}\right.\end{array}$ & $\begin{array}{c}\text { Error } \\
\mathrm{UV} \\
\left.M_{\odot} y r^{-1}\right)\end{array}$ & $\begin{array}{c}\mathrm{SFR} \\
\mathrm{IR} \\
\left(M_{\odot} y r^{-1}\right)\end{array}$ & $\begin{array}{c}\text { SFR } \\
70 \mu m \\
\left(M_{\odot} y r^{-1}\right)\end{array}$ & $\begin{array}{c}\text { Error } \\
70 \mu m \\
\left(M_{\odot} y r^{-1}\right)\end{array}$ & $\begin{array}{c}\text { SFR } \\
24 \mu m \\
\left(M_{\odot} y r^{-1}\right)\end{array}$ & $\begin{array}{c}\text { Error } \\
24 \mu m \\
\left(M_{\odot} y r^{-1}\right)\end{array}$ & $\begin{array}{c}\text { Mass } \\
\text { Stellar Mass } \\
\left(10^{10} M_{\odot}\right)\end{array}$ \\
\hline Abell 2813 & $\ldots$ & $\ldots$ & $\ldots$ & $\ldots$ & $\ldots$ & $\ldots$ & $\ldots$ & $\ldots$ \\
\hline Abell 3084 & 0.64 & 0 & $\ldots$ & $\ldots$ & $\ldots$ & $\ldots$ & $\ldots$ & $\ldots$ \\
\hline Abell 3088 & $\ldots$ & $\ldots$ & $\ldots$ & $\ldots$ & $\ldots$ & $\ldots$ & $\ldots$ & $\ldots$ \\
\hline Abell 3112 & 0.90 & 0 & 2.27 & 0.51 & 0 & 1.26 & 0.13 & 5.53 \\
\hline Abell 3120 & 0.55 & 0 & $\cdots$ & $\ldots$ & $\cdots$ & $\ldots$ & $\ldots$ & $\ldots$ \\
\hline Abell 3158 & 0.60 & 0 & $\ldots$ & $\ldots$ & $\ldots$ & $\ldots$ & $\ldots$ & $\ldots$ \\
\hline Abell 3266 & 0.98 & 0 & $\ldots$ & $\ldots$ & $\ldots$ & $\ldots$ & $\ldots$ & $\ldots$ \\
\hline Abell 3364 & $\ldots$ & $\ldots$ & $\ldots$ & $\ldots$ & $\ldots$ & $\ldots$ & $\ldots$ & $\ldots$ \\
\hline Abell 3376 & 0.29 & 0 & $\ldots$ & $\ldots$ & $\ldots$ & $\ldots$ & $\ldots$ & $\ldots$ \\
\hline Abell 3391 & $\ldots$ & $\ldots$ & 0.54 & 0.13 & 0 & 0.42 & 0.04 & 9.12 \\
\hline Abell 3395 & $\ldots$ & $\ldots$ & $\ldots$ & $\ldots$ & $\ldots$ & $\ldots$ & $\ldots$ & $\ldots$ \\
\hline Abell 3528S & 1.21 & 0 & $\ldots$ & $\ldots$ & $\ldots$ & $\ldots$ & $\ldots$ & $\ldots$ \\
\hline Abell 3558 & 0.82 & 0 & $\ldots$ & $\ldots$ & $\ldots$ & $\ldots$ & $\ldots$ & $\ldots$ \\
\hline Abell 3562 & 0.44 & 0 & $\ldots$ & $\ldots$ & $\ldots$ & $\ldots$ & $\ldots$ & $\ldots$ \\
\hline Abell 3571 & $\ldots$ & $\ldots$ & $\ldots$ & $\ldots$ & $\ldots$ & $\ldots$ & $\ldots$ & $\ldots$ \\
\hline Abell 3581 & 0.21 & 0 & $\ldots$ & $\ldots$ & $\ldots$ & $\ldots$ & $\ldots$ & $\ldots$ \\
\hline Abell 3667 & $\ldots$ & $\ldots$ & $\ldots$ & $\ldots$ & $\ldots$ & $\ldots$ & $\ldots$ & $\ldots$ \\
\hline Abell 3822 & 0.74 & 0 & $\cdots$ & $\ldots$ & $\cdots$ & $\ldots$ & $\ldots$ & $\ldots$ \\
\hline Abell 3827 & 1.70 & 0 & $\ldots$ & $\ldots$ & $\ldots$ & $\ldots$ & $\ldots$ & $\ldots$ \\
\hline Abell 3921 & 1.31 & 0 & $\ldots$ & $\ldots$ & $\ldots$ & $\ldots$ & $\ldots$ & $\ldots$ \\
\hline Abell 4038 & $\ldots$ & $\ldots$ & $\ldots$ & $\ldots$ & $\ldots$ & $\cdots$ & $\ldots$ & $\ldots$ \\
\hline Abell 4059 & 0.66 & 0 & 0.57 & 0.47 & 0.11 & 0.31 & 0.03 & 6.04 \\
\hline Abell S0405 & 0.73 & 0 & $\ldots$ & $\ldots$ & $\ldots$ & $\ldots$ & $\ldots$ & $\ldots$ \\
\hline Abell S0592 & $\ldots$ & $\ldots$ & $\ldots$ & $\ldots$ & $\ldots$ & $\ldots$ & $\ldots$ & $\ldots$ \\
\hline AC 114 & $\ldots$ & $\ldots$ & 4.62 & $\ldots$ & $\ldots$ & 0.95 & 0.10 & 12.25 \\
\hline AWM7 & 0.24 & 0 & $\ldots$ & $\ldots$ & $\ldots$ & $\ldots$ & $\ldots$ & $\ldots$ \\
\hline CENTAURUS & 0.16 & 0 & 0.18 & 0.34 & 0.08 & 0.23 & 0.03 & 6.22 \\
\hline CID 0072 & 0.27 & 0 & 0.26 & 0.17 & 0 & 0.10 & 0.01 & 3.21 \\
\hline CL J1226.9+3332 & $\ldots$ & $\ldots$ & 132.54 & 26.75 & 0 & 3.41 & 0.51 & 28.97 \\
\hline
\end{tabular}


Table 7-Continued

\begin{tabular}{|c|c|c|c|c|c|c|c|c|}
\hline $\begin{array}{c}\text { Name } \\
\text { Cluster Name }\end{array}$ & $\begin{array}{c}\mathrm{SFR} \\
\mathrm{UV} \\
\left(M_{\odot} y r^{-}\right.\end{array}$ & $\begin{array}{c}\text { Error } \\
\mathrm{UV} \\
M_{\odot} y r^{-}\end{array}$ & $\begin{array}{c}\mathrm{SFR} \\
\mathrm{IR} \\
\left(M_{\odot} y r^{-1}\right)\end{array}$ & $\begin{array}{c}\mathrm{SFR} \\
70 \mu m \\
\left(M_{\odot} y r^{-1}\right)\end{array}$ & $\begin{array}{c}\text { Error } \\
70 \mu m \\
\left(M_{\odot} y r^{-1}\right)\end{array}$ & $\begin{array}{c}\text { SFR } \\
24 \mu m \\
\left(M_{\odot} y r^{-1}\right)\end{array}$ & $\begin{array}{c}\text { Error } \\
24 \mu m \\
\left(M_{\odot} y r^{-1}\right)\end{array}$ & $\begin{array}{c}\text { Mass } \\
\text { Stellar Mass } \\
\left(10^{10} M_{\odot}\right)\end{array}$ \\
\hline CYGNUS A & $\cdots$ & $\cdots$ & 95.04 & 31.23 & 6.27 & 167.24 & 16.72 & 7.56 \\
\hline ESO 3060170 & 0.39 & 0 & $\ldots$ & $\ldots$ & $\cdots$ & $\ldots$ & $\ldots$ & $\cdots$ \\
\hline ESO 5520200 & 0.40 & 0 & $\cdots$ & $\cdots$ & $\cdots$ & $\cdots$ & $\cdots$ & $\cdots$ \\
\hline EXO 0422-086 & 0.40 & 0.09 & $\ldots$ & $\ldots$ & $\ldots$ & $\ldots$ & $\ldots$ & $\ldots$ \\
\hline HCG 0062 & $\cdots$ & $\ldots$ & 1.07 & 0.14 & 0 & 0.21 & 0.02 & 3.56 \\
\hline HCG 42 & 0.24 & 0 & 0.32 & 0.06 & 0 & 0.16 & 0.02 & 4.47 \\
\hline HERCULES A & 1.34 & 0 & 1.17 & $\ldots$ & $\cdots$ & 0.40 & 0.06 & 4.32 \\
\hline HYDRA A & 2.62 & 0.19 & 3.77 & 3.08 & 0.73 & 2.13 & 0.21 & 4.88 \\
\hline M49 & 0.06 & 0 & 0.58 & 0.08 & 0.02 & 0.76 & 0.08 & 2.98 \\
\hline M87 & 0.10 & 0 & 0.24 & 0.18 & 0.04 & 0.37 & 0.04 & 4.55 \\
\hline MACS J0011.7-1523 & $\cdots$ & $\cdots$ & $\cdots$ & $\cdots$ & $\cdots$ & $\cdots$ & $\cdots$ & $\cdots$ \\
\hline MACS J0035.4-2015 & $\cdots$ & $\cdots$ & $\cdots$ & $\cdots$ & $\cdots$ & $\cdots$ & $\cdots$ & $\cdots$ \\
\hline MACS J0159.8-0849 & $\cdots$ & $\cdots$ & $\cdots$ & $\cdots$ & $\cdots$ & $\cdots$ & $\cdots$ & $\cdots$ \\
\hline MACS J0242.5-2132 & $\cdots$ & $\cdots$ & $\cdots$ & $\cdots$ & $\cdots$ & $\cdots$ & $\cdots$ & $\cdots$ \\
\hline MACS J0257.1-2325 & $\cdots$ & $\cdots$ & $\cdots$ & $\cdots$ & $\cdots$ & $\cdots$ & $\cdots$ & $\cdots$ \\
\hline MACS J0257.6-2209 & $\cdots$ & $\cdots$ & $\cdots$ & $\cdots$ & $\cdots$ & $\cdots$ & $\cdots$ & $\cdots$ \\
\hline MACS J0308.9+2645 & $\cdots$ & $\cdots$ & $\ldots$ & $\ldots$ & $\cdots$ & $\ldots$ & $\cdots$ & $\cdots$ \\
\hline MACS J0329.6-0211 & $\cdots$ & $\cdots$ & $\cdots$ & $\cdots$ & $\cdots$ & $\cdots$ & $\cdots$ & $\cdots$ \\
\hline MACS J0417.5-1154 & $\cdots$ & $\cdots$ & $\cdots$ & $\cdots$ & $\cdots$ & $\cdots$ & $\cdots$ & $\cdots$ \\
\hline MACS J0429.6-0253 & $\cdots$ & $\cdots$ & $\cdots$ & $\cdots$ & $\cdots$ & $\cdots$ & $\cdots$ & $\cdots$ \\
\hline MACS J0520.7-1328 & $\cdots$ & $\cdots$ & $\cdots$ & $\cdots$ & $\cdots$ & $\cdots$ & $\cdots$ & $\cdots$ \\
\hline MACS J0547.0-3904 & $\cdots$ & $\cdots$ & $\cdots$ & $\cdots$ & $\cdots$ & $\cdots$ & $\cdots$ & $\cdots$ \\
\hline MACS J0717.5+3745 & $\cdots$ & $\cdots$ & $\cdots$ & $\cdots$ & $\cdots$ & $\cdots$ & $\cdots$ & $\cdots$ \\
\hline MACS J0744.8+3927 & $\cdots$ & $\cdots$ & $\cdots$ & $\cdots$ & $\cdots$ & $\cdots$ & $\cdots$ & $\cdots$ \\
\hline MACS J1115.2+5320 & $\cdots$ & $\cdots$ & $\cdots$ & $\cdots$ & $\cdots$ & $\cdots$ & $\cdots$ & $\cdots$ \\
\hline MACS J1115.8+0129 & $\cdots$ & $\cdots$ & $\cdots$ & $\cdots$ & $\cdots$ & $\cdots$ & $\cdots$ & $\cdots$ \\
\hline MACS J1131.8-1955 & $\cdots$ & $\cdots$ & $\cdots$ & $\cdots$ & $\cdots$ & $\cdots$ & $\cdots$ & $\cdots$ \\
\hline MACS J1149.5+2223 & $\cdots$ & $\cdots$ & $\cdots$ & $\cdots$ & $\cdots$ & $\cdots$ & $\cdots$ & $\cdots$ \\
\hline MACS J1206.2-0847 & $\cdots$ & $\cdots$ & $\cdots$ & $\cdots$ & $\cdots$ & $\cdots$ & $\cdots$ & $\cdots$ \\
\hline
\end{tabular}


Table 7-Continued

\begin{tabular}{|c|c|c|c|c|c|c|c|c|}
\hline $\begin{array}{c}\text { Name } \\
\text { Cluster Name }\end{array}$ & $\begin{array}{c}\text { SFR } \\
\mathrm{UV} \\
\left(M_{\odot} y r^{-}\right.\end{array}$ & $\begin{array}{c}\text { Error } \\
\mathrm{UV} \\
\left.M_{\odot} y r^{-1}\right)\end{array}$ & $\begin{array}{c}\text { SFR } \\
\text { IR } \\
\left(M_{\odot} y r^{-1}\right)\end{array}$ & $\begin{array}{c}\text { SFR } \\
70 \mu m \\
\left(M_{\odot} y r^{-1}\right)\end{array}$ & $\begin{array}{c}\text { Error } \\
70 \mu m \\
\left(M_{\odot} y r^{-1}\right)\end{array}$ & $\begin{array}{c}\text { SFR } \\
24 \mu m \\
\left(M_{\odot} y r^{-1}\right)\end{array}$ & $\begin{array}{c}\text { Error } \\
24 \mu m \\
\left(M_{\odot} y r^{-1}\right)\end{array}$ & $\begin{array}{c}\text { Mass } \\
\text { Stellar Mas } \\
\left(10^{10} M_{\odot}\right)\end{array}$ \\
\hline MACS J1311.0-0310 & $\cdots$ & $\cdots$ & $\cdots$ & $\cdots$ & $\cdots$ & $\cdots$ & $\cdots$ & $\cdots$ \\
\hline MACS J1621.3+3810 & $\cdots$ & $\cdots$ & $\cdots$ & $\cdots$ & $\cdots$ & $\cdots$ & $\cdots$ & $\cdots$ \\
\hline MACS J1931.8-2634 & $\cdots$ & $\cdots$ & $\cdots$ & $\cdots$ & $\cdots$ & $\cdots$ & $\cdots$ & $\cdots$ \\
\hline MACS J2049.9-3217 & $\cdots$ & $\cdots$ & $\cdots$ & $\cdots$ & $\cdots$ & $\cdots$ & $\cdots$ & $\cdots$ \\
\hline MACS J2211.7-0349 & $\cdots$ & $\cdots$ & $\cdots$ & $\cdots$ & $\cdots$ & $\cdots$ & $\cdots$ & $\cdots$ \\
\hline MACS J2214.9-1359 & $\cdots$ & $\cdots$ & $\cdots$ & $\cdots$ & $\cdots$ & $\cdots$ & $\cdots$ & $\cdots$ \\
\hline MACS J2228+2036 & $\cdots$ & $\cdots$ & $\cdots$ & $\cdots$ & $\cdots$ & $\cdots$ & $\cdots$ & $\cdots$ \\
\hline MACS J2229.7-2755 & 6.95 & 1.36 & $\cdots$ & $\cdots$ & $\cdots$ & $\cdots$ & $\cdots$ & $\cdots$ \\
\hline MACS J2245.0+2637 & $\cdots$ & $\ldots$ & $\cdots$ & $\cdots$ & $\cdots$ & $\cdots$ & $\cdots$ & $\cdots$ \\
\hline MKW3S & 0.37 & 0 & $\cdots$ & $\cdots$ & $\cdots$ & $\cdots$ & $\cdots$ & $\cdots$ \\
\hline MKW 04 & 0.33 & 0 & 0.03 & 0.10 & 0 & 0.10 & 0.01 & 5.66 \\
\hline MKW 08 & 0.19 & 0 & $\cdots$ & $\ldots$ & $\cdots$ & $\cdots$ & $\cdots$ & $\cdots$ \\
\hline MS 0016.9+1609 & $\cdots$ & $\cdots$ & $\cdots$ & $\cdots$ & $\cdots$ & $\cdots$ & $\cdots$ & $\cdots$ \\
\hline MS 0116.3-0115 & 0.48 & 0 & $\cdots$ & $\cdots$ & $\cdots$ & $\cdots$ & $\cdots$ & $\cdots$ \\
\hline MS $0440.5+0204$ & $\cdots$ & $\cdots$ & $\cdots$ & $\cdots$ & $\cdots$ & $\cdots$ & $\cdots$ & $\cdots$ \\
\hline MS 0451.6-0305 & $\ldots$ & $\cdots$ & 8.09 & 7.63 & 0 & 2.79 & 0.42 & 11.00 \\
\hline MS 0735.6+7421 & 1.51 & 0 & $\cdots$ & $\cdots$ & $\cdots$ & $\cdots$ & $\cdots$ & $\cdots$ \\
\hline MS 0839.8+2938 & 1.20 & 0 & $\cdots$ & $\cdots$ & $\cdots$ & $\cdots$ & $\cdots$ & $\cdots$ \\
\hline MS 0906.5+1110 & $\cdots$ & $\cdots$ & $\cdots$ & $\cdots$ & $\cdots$ & $\cdots$ & $\cdots$ & $\cdots$ \\
\hline MS $1006.0+1202$ & $\cdots$ & $\cdots$ & $\cdots$ & $\cdots$ & $\cdots$ & $\cdots$ & $\cdots$ & $\cdots$ \\
\hline MS 1008.1-1224 & $\cdots$ & $\ldots$ & $\cdots$ & $\cdots$ & $\cdots$ & $\ldots$ & $\cdots$ & $\ldots$ \\
\hline MS $1455.0+2232$ & 5.90 & 0.86 & 9.46 & $\cdots$ & $\cdots$ & 3.16 & 0.47 & 10.86 \\
\hline MS 2137.3-2353 & 3.09 & 0 & 18.49 & $\cdots$ & $\cdots$ & 3.73 & 0.84 & 10.96 \\
\hline MS J1157.3+5531 & 0.69 & 0 & $\ldots$ & $\cdots$ & $\cdots$ & $\cdots$ & $\cdots$ & $\cdots$ \\
\hline NGC 0507 & 0.26 & 0 & 0.29 & 0.05 & 0 & 0.17 & 0.02 & 4.83 \\
\hline NGC 4636 & 0.02 & 0 & -0.01 & 0.07 & 0.02 & 0.02 & 0 & 1.38 \\
\hline NGC 5044 & 0.12 & 0 & 0.27 & 0.23 & 0.05 & 0.19 & 0.02 & 3.61 \\
\hline NGC 5813 & 0.07 & 0 & 0.04 & 0.11 & 0.04 & 0.10 & 0.01 & 2.40 \\
\hline NGC 5846 & 0.08 & 0 & 0.14 & 0.17 & 0.04 & 0.14 & 0.01 & 4.78 \\
\hline
\end{tabular}


Table 7-Continued

\begin{tabular}{|c|c|c|c|c|c|c|c|c|}
\hline $\begin{array}{c}\text { Name } \\
\text { Cluster Name }\end{array}$ & $\begin{array}{c}\mathrm{SFR} \\
\mathrm{UV} \\
\left(M_{\odot} y r^{-1}\right.\end{array}$ & $\begin{array}{c}\text { Error } \\
\mathrm{UV} \\
M_{\odot} y r^{-}\end{array}$ & $\begin{array}{c}\mathrm{SFR} \\
\mathrm{IR} \\
\left(M_{\odot} y r^{-1}\right)\end{array}$ & $\begin{array}{c}\text { SFR } \\
70 \mu m \\
\left(M_{\odot} y r^{-1}\right)\end{array}$ & $\begin{array}{c}\text { Error } \\
70 \mu m \\
\left(M_{\odot} y r^{-1}\right)\end{array}$ & $\begin{array}{c}\mathrm{SFR} \\
24 \mu m \\
\left(M_{\odot} y r^{-1}\right)\end{array}$ & $\begin{array}{c}\text { Error } \\
24 \mu m \\
\left(M_{\odot} y r^{-1}\right)\end{array}$ & $\begin{array}{c}\text { Mass } \\
\text { Stellar Mass } \\
\left(10^{10} M_{\odot}\right)\end{array}$ \\
\hline OPHIUCHUS & 2.60 & 0.10 & $\cdots$ & $\cdots$ & $\cdots$ & $\cdots$ & $\cdots$ & $\cdots$ \\
\hline PKS 0745-191 & $\ldots$ & $\ldots$ & 17.81 & 11.52 & 2.57 & 8.50 & 0.85 & 9.51 \\
\hline RBS 0461 & $\ldots$ & $\ldots$ & $\ldots$ & $\ldots$ & $\ldots$ & $\ldots$ & $\ldots$ & $\ldots$ \\
\hline RBS 0533 & 0.14 & 0 & $\cdots$ & $\cdots$ & $\cdots$ & $\cdots$ & $\cdots$ & $\cdots$ \\
\hline RBS 0797 & $\cdots$ & $\cdots$ & $\cdots$ & $\cdots$ & $\cdots$ & $\cdots$ & $\cdots$ & $\cdots$ \\
\hline RCS J2327-0204 & $\cdots$ & $\cdots$ & $\cdots$ & $\cdots$ & $\cdots$ & $\cdots$ & $\cdots$ & $\cdots$ \\
\hline RXCJ0331.1-2100 & $\cdots$ & $\cdots$ & $\cdots$ & $\cdots$ & $\cdots$ & $\cdots$ & $\cdots$ & $\cdots$ \\
\hline RXC J1023.8-2715 & 0.35 & 0.08 & $\ldots$ & $\ldots$ & $\ldots$ & $\ldots$ & $\ldots$ & $\ldots$ \\
\hline RX J0220.9-3829 & $\cdots$ & $\cdots$ & $\cdots$ & $\cdots$ & $\cdots$ & $\cdots$ & $\cdots$ & $\cdots$ \\
\hline RX J0232.2-4420 & $\cdots$ & $\cdots$ & $\ldots$ & $\ldots$ & $\cdots$ & $\cdots$ & $\cdots$ & $\cdots$ \\
\hline RX J0439+0520 & $\cdots$ & $\cdots$ & 9.93 & 7.35 & 1.54 & 7.57 & 0.83 & 8.79 \\
\hline RX J0439.0+0715 & $\cdots$ & $\cdots$ & $\cdots$ & $\cdots$ & $\cdots$ & $\cdots$ & $\cdots$ & $\cdots$ \\
\hline RX J0528.9-3927 & $\cdots$ & $\cdots$ & $\cdots$ & $\cdots$ & $\cdots$ & $\cdots$ & $\cdots$ & $\cdots$ \\
\hline RX J0647.7+7015 & $\cdots$ & $\cdots$ & $\cdots$ & $\cdots$ & $\cdots$ & $\cdots$ & $\cdots$ & $\cdots$ \\
\hline RX J0819.6+6336 & $\ldots$ & $\cdots$ & $\cdots$ & $\ldots$ & $\ldots$ & $\cdots$ & $\cdots$ & $\ldots$ \\
\hline RX J1000.4+4409 & $\ldots$ & $\cdots$ & $\cdots$ & $\cdots$ & $\cdots$ & $\cdots$ & $\cdots$ & $\cdots$ \\
\hline RX J1022.1+3830 & 0.34 & 0 & $\cdots$ & $\ldots$ & $\cdots$ & $\cdots$ & $\ldots$ & $\ldots$ \\
\hline RX J1130.0+3637 & 0.20 & 0 & $\cdots$ & $\cdots$ & $\cdots$ & $\cdots$ & $\cdots$ & $\cdots$ \\
\hline RX J1320.2+3308 & $\ldots$ & $\cdots$ & $\cdots$ & $\cdots$ & $\cdots$ & $\cdots$ & $\cdots$ & $\cdots$ \\
\hline RX J1347.5-1145 & $\ldots$ & $\ldots$ & $\ldots$ & $\ldots$ & $\cdots$ & $\cdots$ & $\ldots$ & $\ldots$ \\
\hline RX J1423.8+2404 & $\ldots$ & $\ldots$ & $\cdots$ & $\cdots$ & $\cdots$ & $\cdots$ & $\cdots$ & $\cdots$ \\
\hline RX J1504.1-0248 & 56.50 & 0.46 & $\ldots$ & $\ldots$ & $\ldots$ & $\ldots$ & $\ldots$ & $\ldots$ \\
\hline RX J1532.9+3021 & $\ldots$ & $\ldots$ & 227.52 & 110.14 & 22.03 & 43.54 & 4.35 & 9.33 \\
\hline RX J1539.5-8335 & $\cdots$ & $\cdots$ & $\ldots$ & $\ldots$ & $\ldots$ & $\ldots$ & $\cdots$ & $\cdots$ \\
\hline RX J1720.1+2638 & 2.67 & 0.68 & $\cdots$ & $\cdots$ & $\cdots$ & $\cdots$ & $\cdots$ & $\cdots$ \\
\hline RX J1720.2+3536 & $\ldots$ & $\ldots$ & $\ldots$ & $\ldots$ & $\cdots$ & $\cdots$ & $\ldots$ & $\ldots$ \\
\hline RX J1852.1+5711 & $\ldots$ & $\ldots$ & $\ldots$ & $\ldots$ & $\ldots$ & $\ldots$ & $\ldots$ & $\ldots$ \\
\hline RX J2129.6+0005 & 2.09 & 0 & 5.58 & 4.71 & 0 & 5.15 & 0.51 & 8.61 \\
\hline SC 1327-312 & 0.37 & 0 & $\ldots$ & $\ldots$ & $\ldots$ & $\ldots$ & $\ldots$ & $\ldots$ \\
\hline
\end{tabular}


Table 7 - Continued

\begin{tabular}{|c|c|c|c|c|c|c|c|c|}
\hline $\begin{array}{c}\text { Name } \\
\text { Cluster Name }\end{array}$ & $\begin{array}{c}\mathrm{SFR} \\
\mathrm{UV} \\
\left(M_{\odot} y r^{-1}\right. \\
\end{array}$ & $\begin{array}{c}\text { Error } \\
\mathrm{UV} \\
\left.M_{\odot} y r^{-1}\right)\end{array}$ & $\begin{array}{c}\mathrm{SFR} \\
\mathrm{IR} \\
\left(M_{\odot} y r^{-1}\right)\end{array}$ & $\begin{array}{c}\text { SFR } \\
70 \mu m \\
\left(M_{\odot} y r^{-1}\right) \\
\end{array}$ & $\begin{array}{c}\text { Error } \\
70 \mu m \\
\left(M_{\odot} y r^{-1}\right) \\
\end{array}$ & $\begin{array}{c}\text { SFR } \\
24 \mu m \\
\left(M_{\odot} y r^{-1}\right) \\
\end{array}$ & $\begin{array}{c}\text { Error } \\
24 \mu m \\
\left(M_{\odot} y r^{-1}\right) \\
\end{array}$ & $\begin{array}{c}\text { Mass } \\
\text { Stellar Mass } \\
\left(10^{10} M_{\odot}\right) \\
\end{array}$ \\
\hline SERSIC 159-03 & 0.52 & 0 & 0.89 & 0.67 & 0.15 & 0.30 & 0.03 & 4.21 \\
\hline SS2B153 & 0.28 & 0 & $\cdots$ & $\cdots$ & $\cdots$ & $\cdots$ & $\cdots$ & $\cdots$ \\
\hline UGC 03957 & 0.49 & 0 & $\ldots$ & $\ldots$ & $\ldots$ & $\ldots$ & $\ldots$ & $\ldots$ \\
\hline UGC 12491 & $\ldots$ & $\ldots$ & $\ldots$ & $\ldots$ & $\ldots$ & $\ldots$ & $\ldots$ & $\ldots$ \\
\hline ZWCL 1215 & $\ldots$ & $\ldots$ & $\ldots$ & $\ldots$ & $\ldots$ & $\ldots$ & $\ldots$ & $\ldots$ \\
\hline ZWCL $1358+6245$ & $\cdots$ & $\cdots$ & 2.86 & $\cdots$ & $\cdots$ & 1.73 & 0.26 & 5.36 \\
\hline ZWCL 1742 & $\ldots$ & $\ldots$ & 2.02 & 1.52 & 0.32 & 1.45 & 0.15 & 3.80 \\
\hline ZWCL 1953 & $\ldots$ & $\cdots$ & $\ldots$ & $\ldots$ & $\ldots$ & $\ldots$ & $\cdots$ & $\cdots$ \\
\hline ZWCL 3146 & 20.86 & 1.05 & 65.51 & 34.09 & 10.32 & 31.28 & 3.13 & 9.98 \\
\hline ZWICKY 2701 & $\ldots$ & $\ldots$ & 0.97 & 2.91 & 0 & 0.57 & 0.14 & 6.32 \\
\hline $\mathrm{ZwCl} 0857.9+2107$ & 5.93 & 0.55 & 428.41 & 96.25 & 19.25 & 164.99 & 16.50 & 2.56 \\
\hline
\end{tabular}

Note. - A star formation rate uncertainty of 0 identifies the quoted rate as a $3 \sigma$ upper limit.

Note. - UV SFR are calculated for all objects, including those in high $\mathrm{K}_{0}$ systems. Upper limits are then calculated using the uncertainties on the NUV, K, and inert BCG color. If a star formation rate is consistent with a SFR of zero within $3 \sigma$, a $3 \sigma$ upper limit is reported.

Note. - The IR SFR is estimated by a fit to the Groves et al. (2008) models. The 24 and 70 micron SFRs are estimated from empirical relationships reported in Calzetti et al. (2010) and the uncertainties are calculated using the 24 and 70 micron flux uncertainties, respectively. 


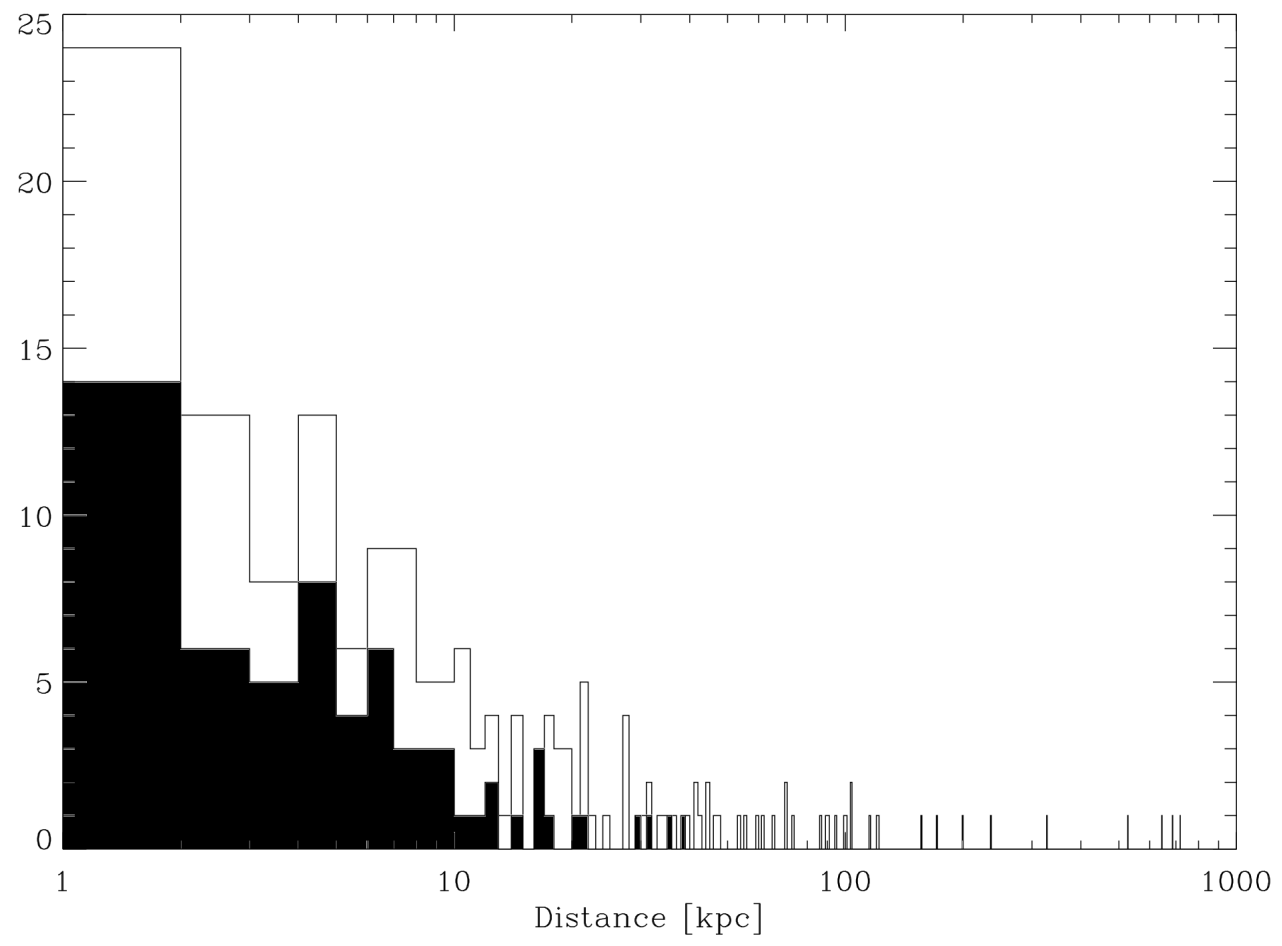

Fig. 1. - The projected physical distance, in $\mathrm{kpc} \mathrm{h}_{70}^{-1}$, between X-ray centroid and the BCG we identified. All BCGs with an RA and Dec, not just those with GALEX and Spitzer data are plotted here. The the shaded region highlights BCGs in low $\mathrm{K}_{0}$ clusters. In high central entropy systems, $37 \%$ of BCGs lie within $10 \mathrm{kpc}$ of the X-ray centroid, while the percentage is increased to $74 \%$ for low central entropy systems. All BCGs which lie greater than $40 \mathrm{kpc}$ away from their X-ray centroid are in high $\mathrm{K}_{0}$ clusters. 

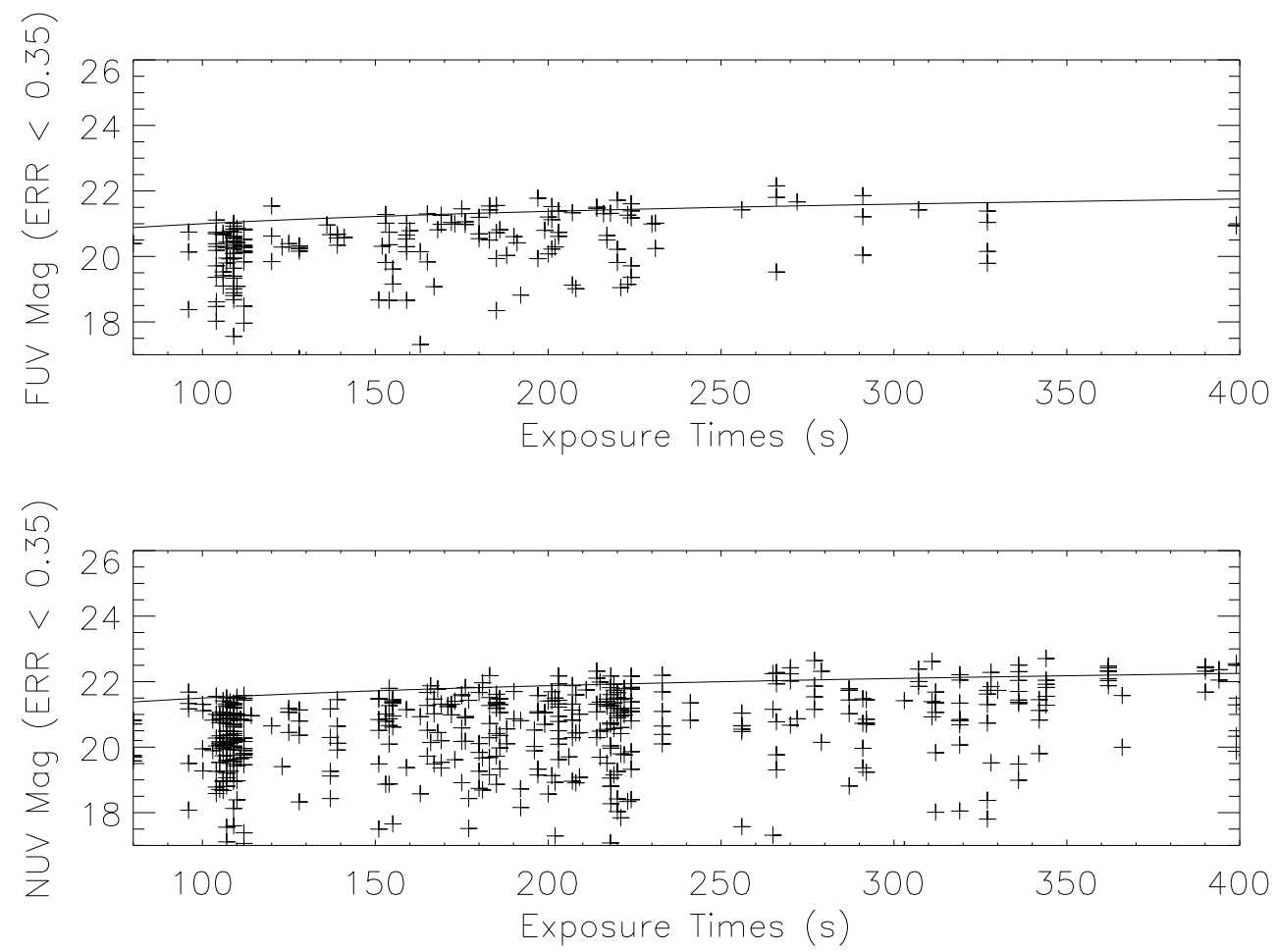

Fig. 2.- The UV magnitudes (AB scale) for all UV sources within $1^{\prime}$ of the BCG locations (regardless of identity) with flux uncertainties less than 0.35 magnitudes. The upper envelope of this distribution serves as a basis for estimating the upper limit fluxes for undetected or poorlydetected BCGs for exposure times less than 400 seconds: $F U V_{U L}=18.5+1.25 \log _{10} t$ and $N U V_{U L}=$ $19.0+1.25 \log _{10} t$. We considered all GALEX detections with magnitude errors $>0.35$ to be poorly detected. 


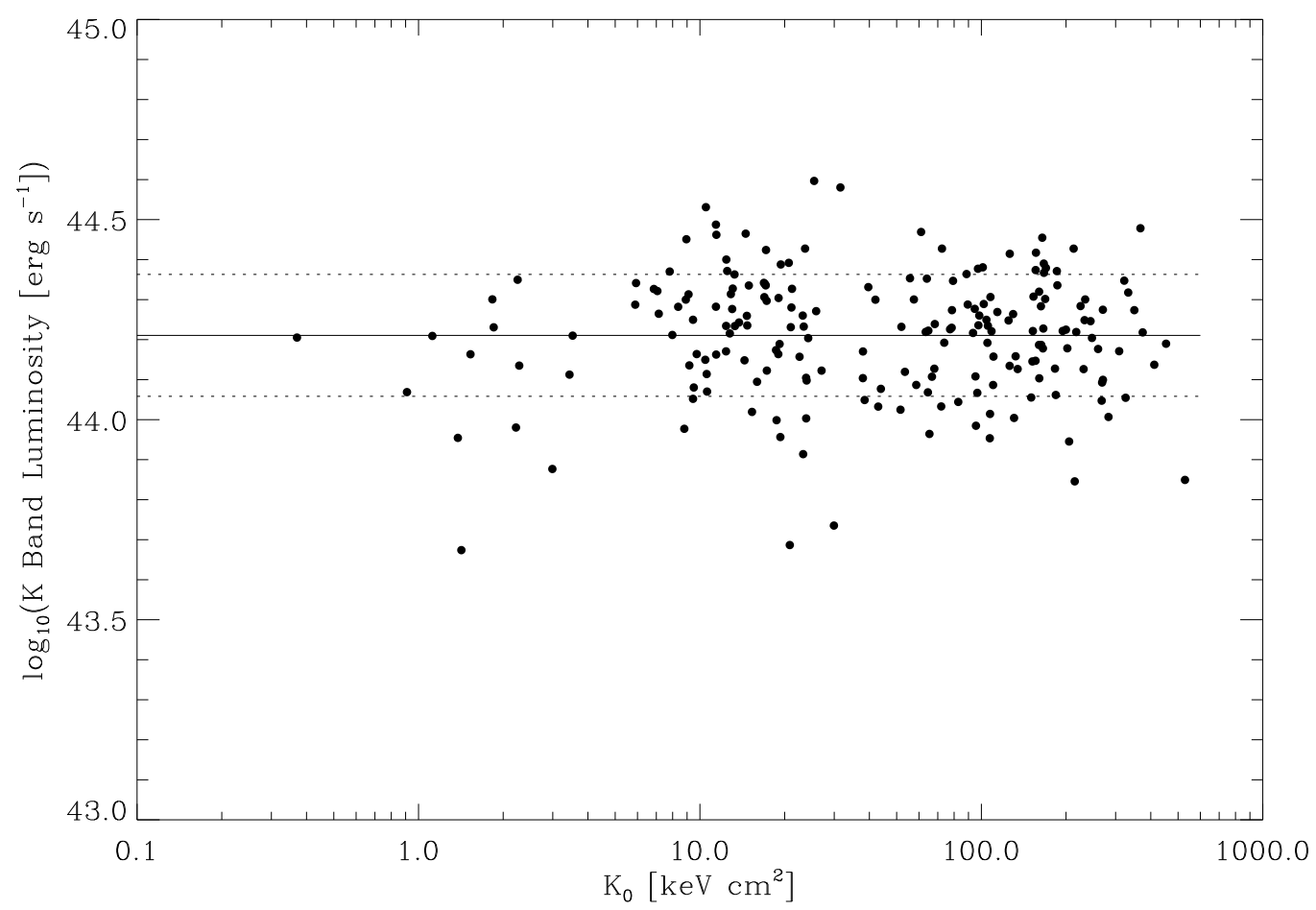

Fig. 3.- The logarithm of $\mathrm{K}$ band luminosity and central entropy of the cluster. The $\mathrm{K}$ band luminosity is calculated from the flux inside $14.3 \mathrm{kpc} \mathrm{h}_{70}^{-1} \mathrm{kpc}$ radius. The luminosities are $\mathrm{k}$ corrected assuming passive evolution. The solid horizontal line represents the mean $\left(1.6 \times 10^{44} \mathrm{erg}\right.$ $\left.\mathrm{s}^{-1} \mathrm{~h}_{70}^{-2}\right)$ of the data points while the dotted lines are the $1 \sigma$ error $\left(+0.7 \times 10^{44} \mathrm{erg} \mathrm{s}^{-1} \mathrm{~h}_{70}^{-2},-0.4 \times\right.$ $\left.10^{44} \mathrm{erg} \mathrm{s}^{-1} \mathrm{~h}_{70}^{-2}\right)$ on the mean. 


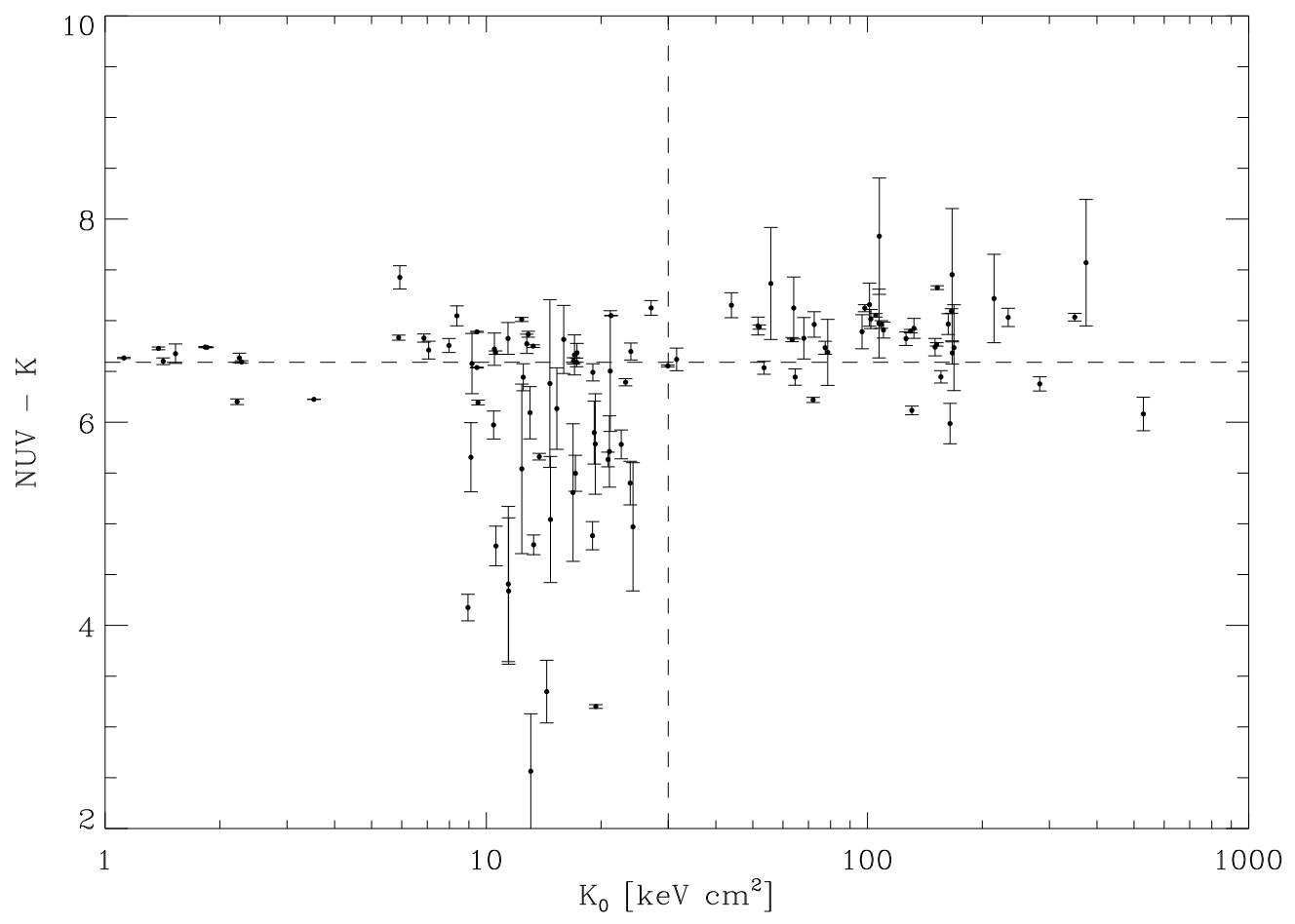

Fig. 4.- NUV-K as a function of cluster central entropy. The vertical dashed line is at $30 \mathrm{keV} \mathrm{cm}^{2}$, our cutoff for the definition of low entropy clusters. Note the large color distribution for low $\mathrm{K}_{0}$ objects, while the high entropy objects have a more consistent redder color. The $\mathrm{K}$ band fluxes have been k-corrected assuming passive evolution. The horizontal dashed line represents a NUV-K color of 6.59 magnitudes, the mean of the BCGs in clusters with with $\mathrm{K}_{0} \leq 30 \mathrm{keV} \mathrm{cm}^{2}$. There may appear to be a trend with the low entropy objects, but this is a selection effect where the lowest entropy objects that are observed are also the nearest objects. 

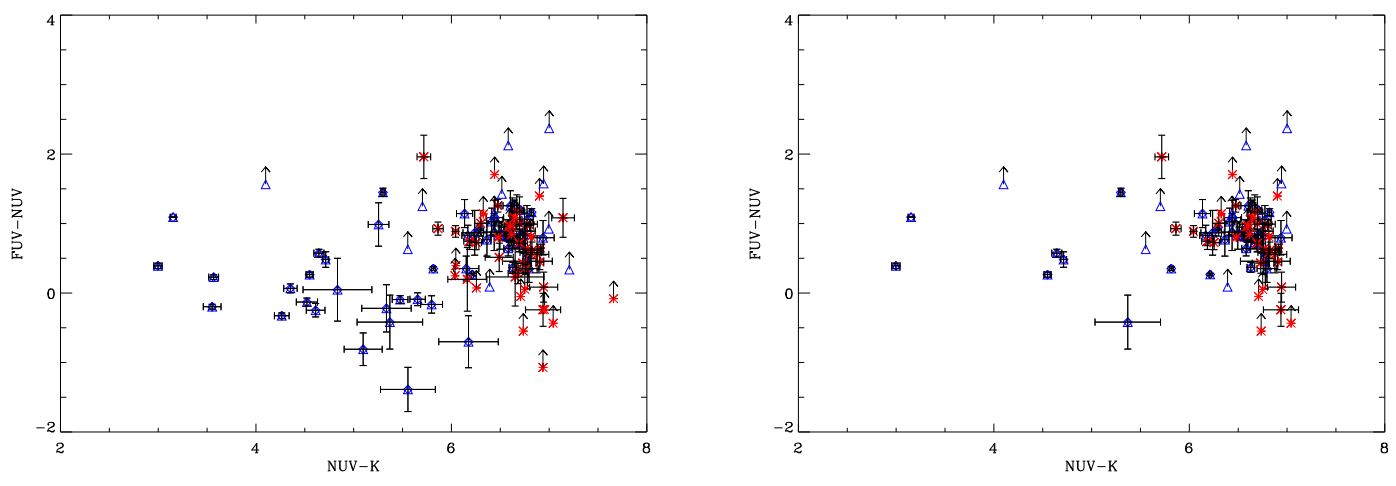

Fig. 5.- FUV-NUV and NUV-K colors. The blue triangles are BCGs in low $\mathrm{K}_{0}$ clusters $(\leq$ $30 \mathrm{keV} \mathrm{cm}^{2}$ ) while the red asterisks are BCGs in high entropy clusters. The left plot includes all of the BCGs while the right plot only includes nearby $(\mathrm{z}<0.15)$ BCGs demonstrating that the bluest FUV-NUV colors, in the left hand plot, are likely arising because of contributions from Ly $\alpha$.
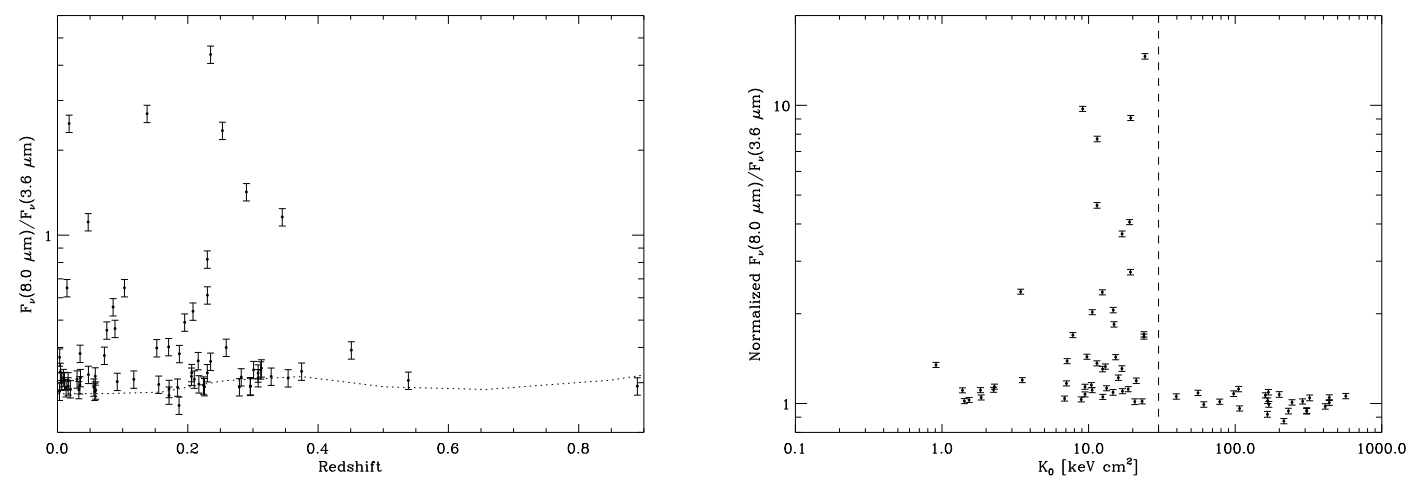

Fig. 6.- Redshift dependence of $8.0 \mu \mathrm{m}$ to $3.6 \mu \mathrm{m}$ ratio. The dotted line represents the expected flux ratio for passively evolving stellar population that is $10 \mathrm{Gyr}$ at $z=0$. While IRS spectra of $\mathrm{H} \alpha$ emitting BCGs show PAH features that would fall in the 8.0 micron bandpass (e.g. Donahue et al. 2011) the observed IRAC 8.0 micron color is sensitive to only strong PAH features. On the right, the flux ratio has been normalized by the passive evolution model and is plotted against the central entropy of the cluster. The dotted line identifies the threshold $30 \mathrm{keV} \mathrm{cm}{ }^{2}$. There appears to be a deficit of excess-IR emitters in the low $\mathrm{K}_{0}$ clusters, but this deficit is likely to be a selection effect since low $K_{0}$ can only be resolved in the most nearby groups. 

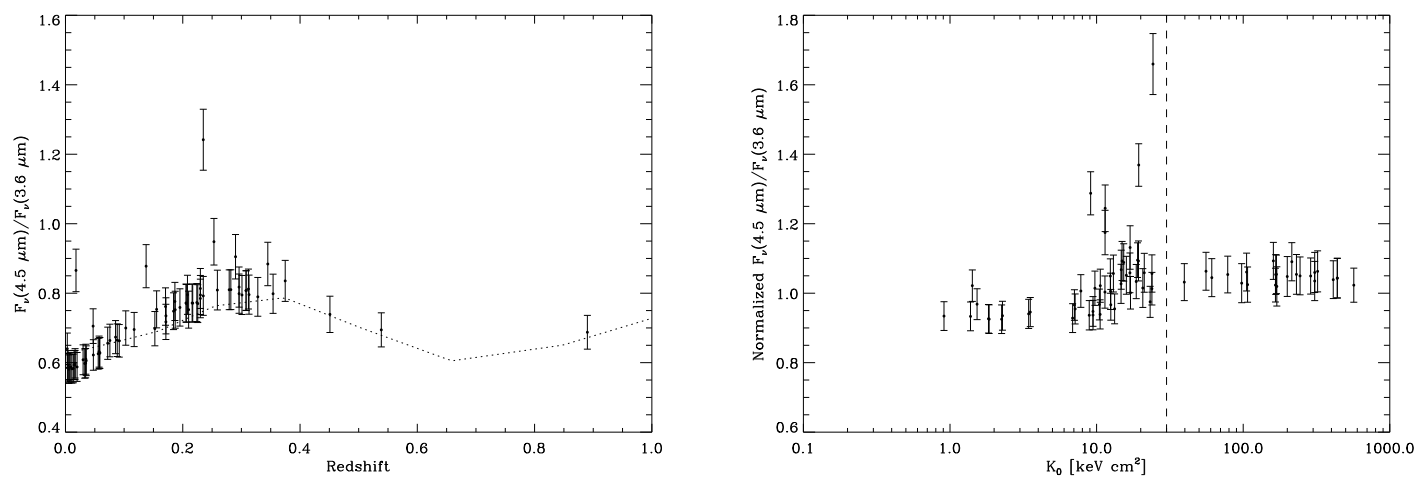

Fig. 7.- The left hand figure shows the flux ratio between the $4.5 \mu \mathrm{m}$ and the $3.6 \mu \mathrm{m}$ flux is plotted as a function of redshift. The dotted line indicates a Starburst99 model for a passively evolving elliptical galaxy with a primarily old stellar population dominated by red giants, with an age of about $10 \mathrm{Gyr}$ at $z=0$. For most of the BCGs the IRAC $4.5 \mu \mathrm{m}$ to $3.6 \mu \mathrm{m}$ colors are consistent with those of a passively evolving population. In the figure on the right, the flux ratio has been normalized by the passive evolution model and is plotted against the central entropy of the cluster. The dotted line again identifies $K_{0}=30 \mathrm{keV} \mathrm{cm}{ }^{2}$. It is interesting that the handful of BCGs (Abell 426, Abell 1068, Abell 1835, and $\mathrm{ZwCl} \mathrm{0857.9+2107)} \mathrm{with} \mathrm{large} \mathrm{excess} 4.5$ micron emission are located only in clusters with $K_{0}$ less than the threshold. 


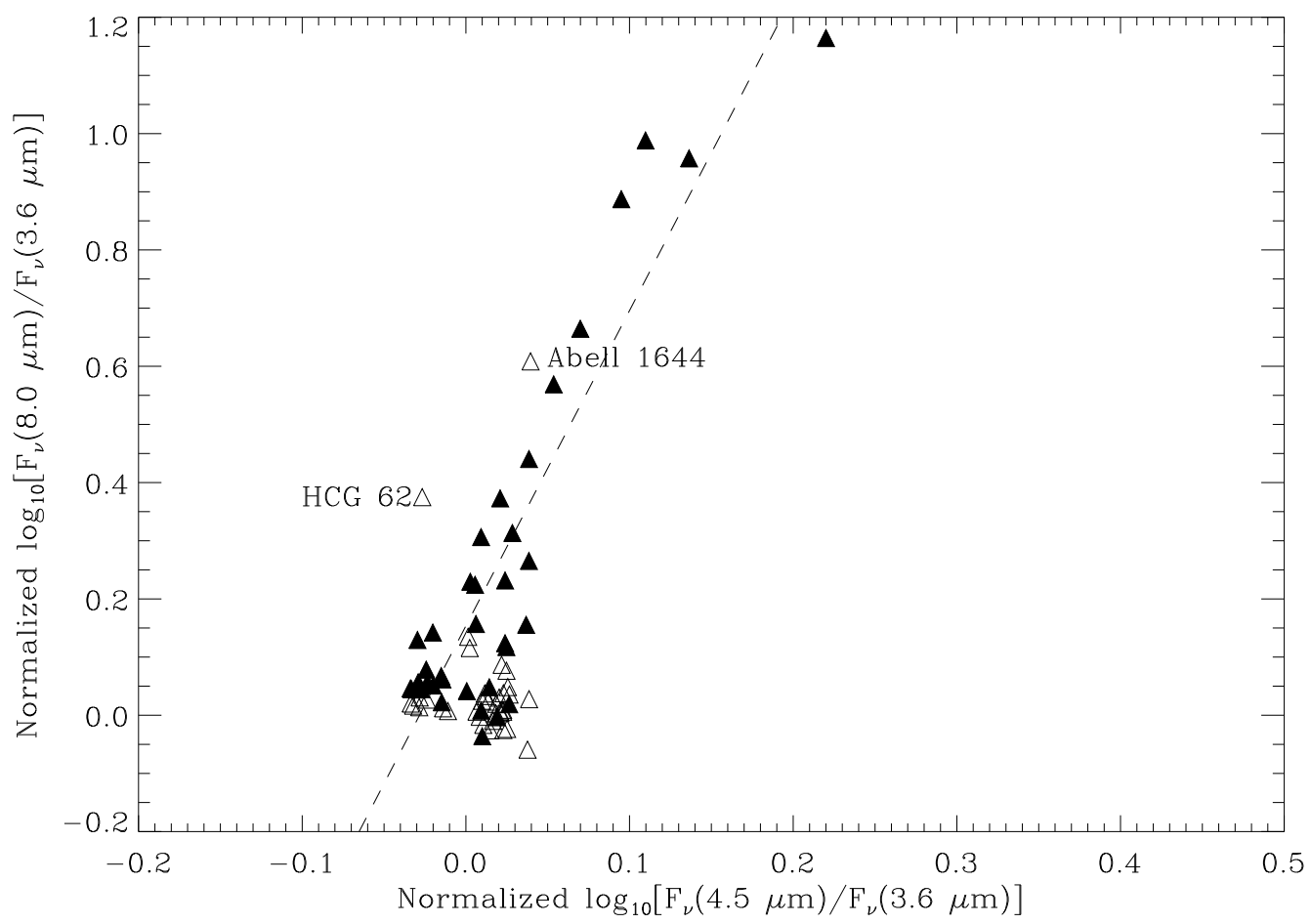

Fig. 8.- 8.0 micron to 3.6 micron ratio and 4.5 micron to 3.6 micron ratio. Both ratios have been normalized for passive evolution. The 8.0/3.6 and the 4.5/3.6 ratios are strongly correlated $(r=0.92(15 \sigma)$ for objects with mid-IR detections and/or NUV-K excesses (shown as filled in triangles), which is expected if the excess 4.5 micron emission is generated by processes related to that producing the 8.0 micron emission. Dashed line is a fit to the data; see text. Abell 1644 and HCG 62 do not have blue NUV-K colors, HCG 62 is a 70 micron upper limit, and Abell 1644 wasn't observed by MIPS. Since these ratios use only IRAC data, the uncertainties in the absolute flux calibration are not included. As long as the IRAC calibration was consistent over time, these are precise relative flux ratios. The absolute flux ratios are accurate to about $2 \%$. 


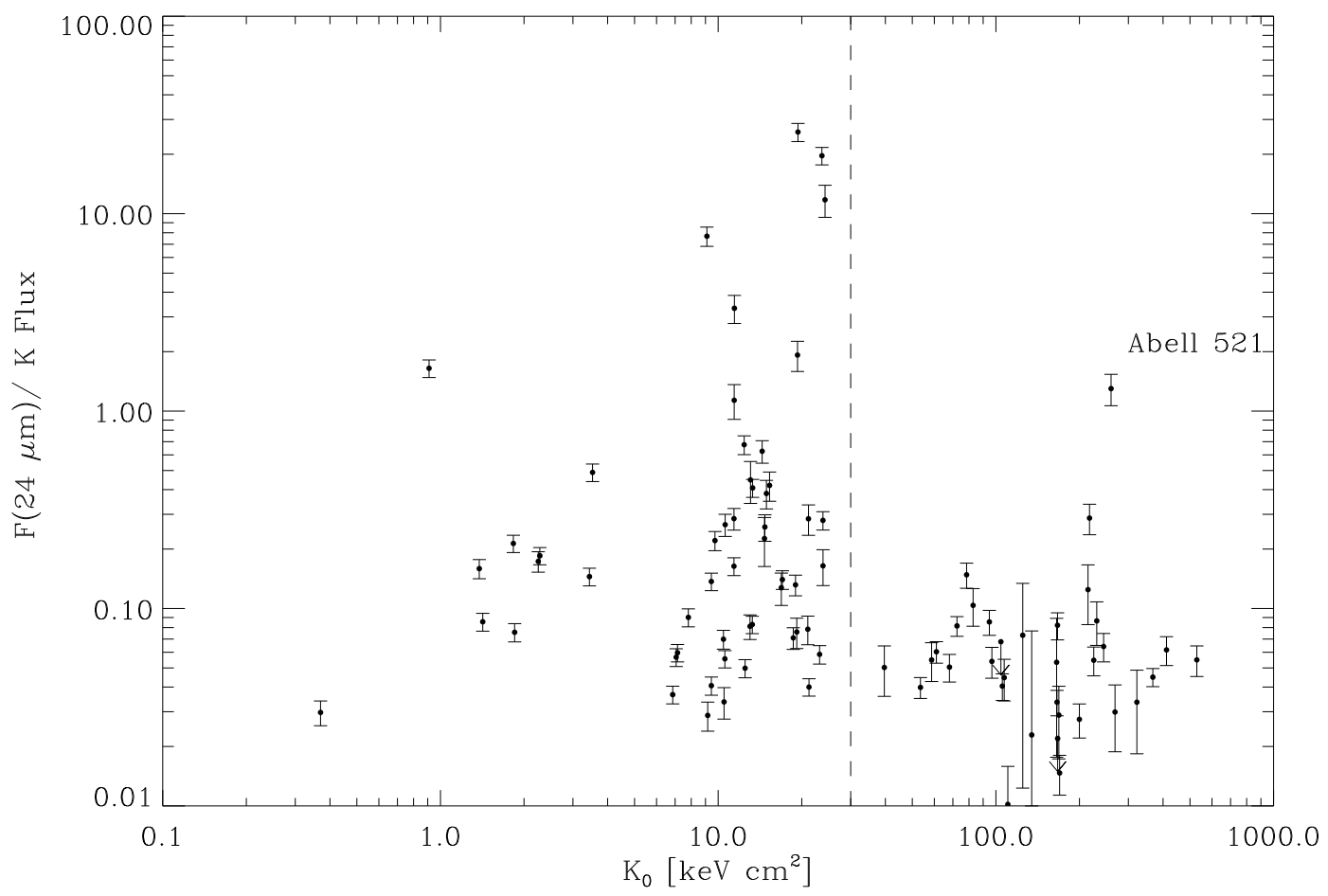

Fig. 9.- 24 micron flux to $\mathrm{K}$ band flux ratio with central entropy of the cluster. BCGs with excess 24 micron flux inhabit clusters with low $K_{0}$, with the exception of Abell 521. Even though it is a high $\mathrm{K}_{0}$ cluster, the BCG in Abell 521 is in a low entropy, compact, X-ray corona (i.e. a "mini-cooling core") which can be associated with BCGs with radio sources and star-formation activity, like BCGs in low $\mathrm{K}_{0}$ clusters of galaxies (Ferrari et al. 2006). 


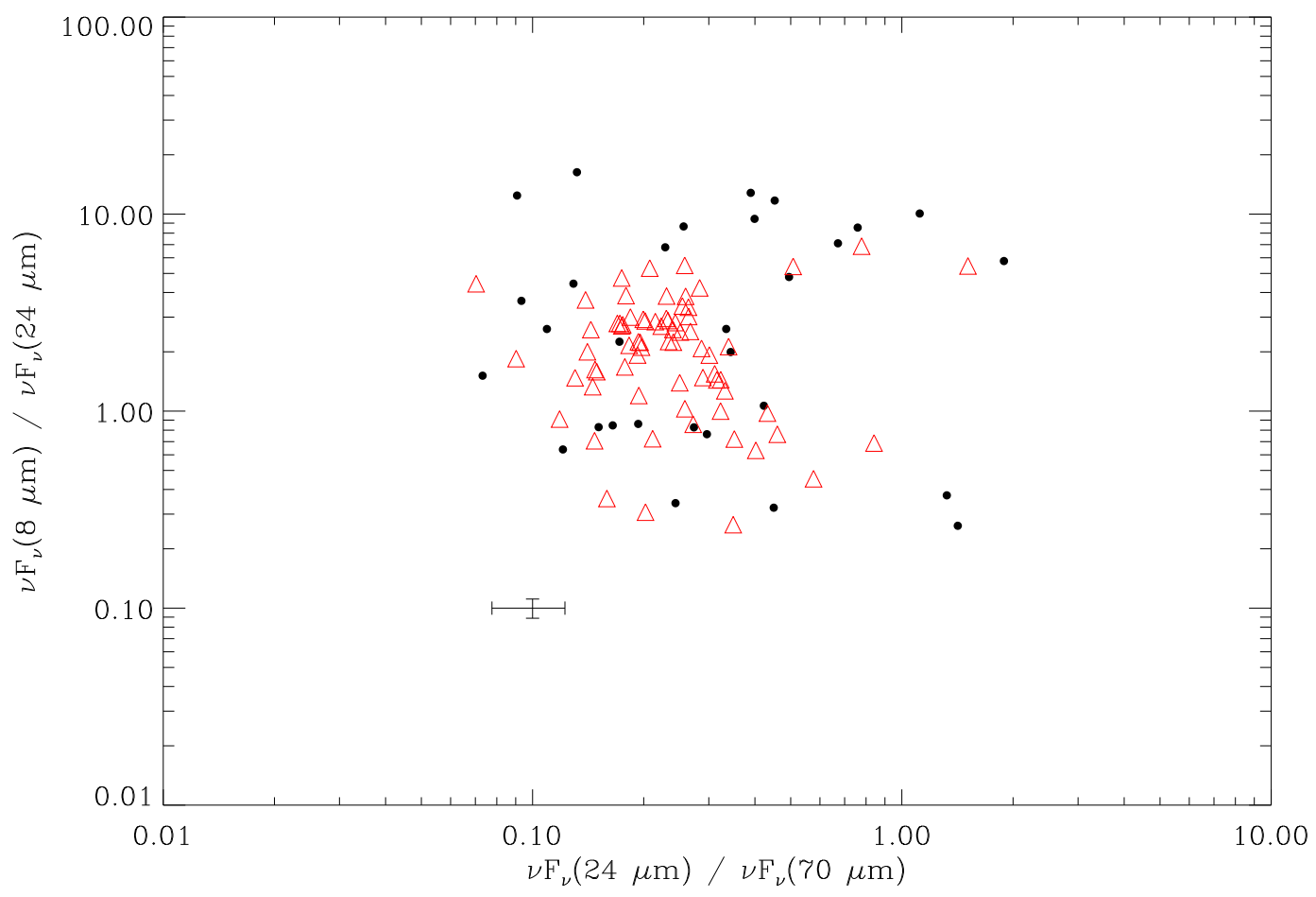

Fig. 10. - Mid IR flux ratio comparison with SINGS galaxies. This plot is similar to Figure 1 in Johnson et al. (2007a). The black dots are our BCGs and the SINGS galaxies (Kennicutt et al. 2003) are overplotted as red triangles. Ratios for objects with MIPS upper limits at 24 or 70 microns are not plotted but are consistent with the distribution of the detected galaxies. An error bar, representing the standard IRAC and MIPS systematic errors, is plotted to represent a typical error bar. Some nearby BCGs have slightly higher 8.0/24 micron flux ratios than SINGS galaxies, but similar 24/70 micron flux ratios. 


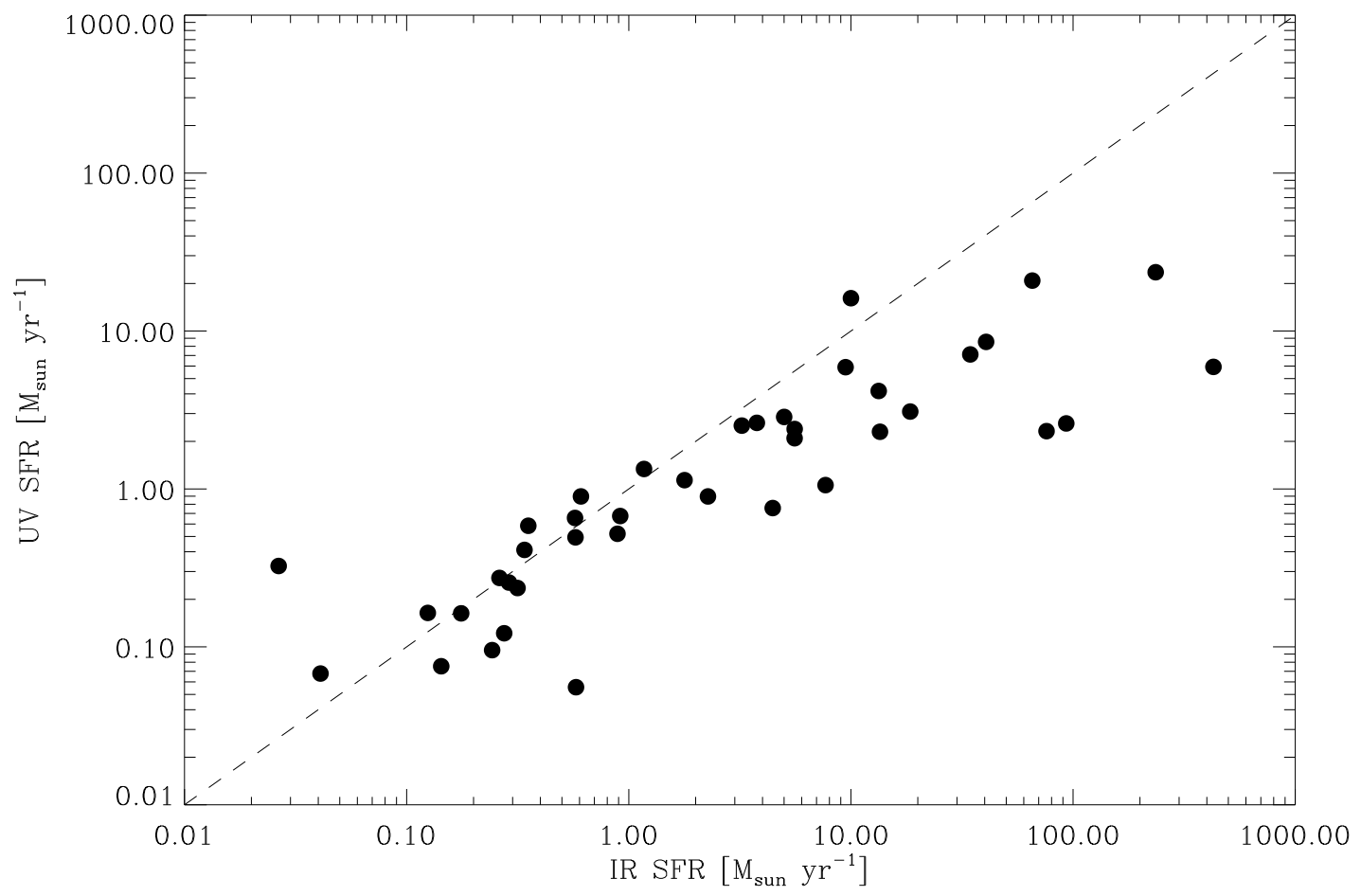

Fig. 11.- UV and IR SFR. The dotted line represents a line of unity. The UV SFR assumes a constant rate of star formation. The model-derived IR star formation rates are consistent with star formation rates measured with a MIPS $70 \mu \mathrm{m}$ SFR estimate as shown in Figure 12. Those objects which fall below the line, BCGs with excess IR star formation, are similar to starburst galaxies, in the sense that for the most luminous star-forming galaxies, most of the star-formation is obscured. 

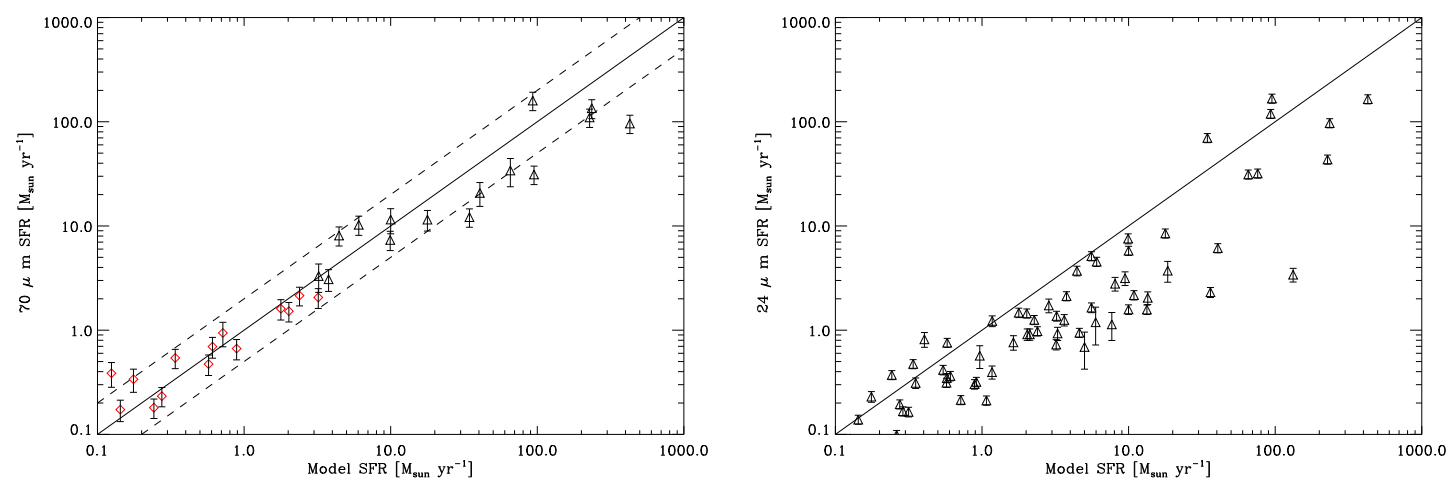

Fig. 12.- Comparison of model IR SFR to single band IR SFR. We compare the estimates from the Groves model star formation rates to the star formation rate estimates using the 70 micron luminosity in the left plot. The line represents a line of unity, not a fit. The dotted lines represent the boundary for a difference of a factor of two in star formation rate. The black triangles represent the high luminosity relation given in Calzetti et al. (2010) while the red diamonds use their relation for galaxies with low IR luminosities (and therefore a larger amount of the IR flux is produced by dust heated by evolved stars rather than hot stars). The right plot is a similar plot relating the 24 micron luminosity to the Groves model star formation rates. The 24 micron SFRs tend to have lower estimates as the 70 micron luminosities are found nearer to the peak of the dust blackbody and therefore more representative of the total IR luminosity and SFR. For the most luminous 70 microns galaxies, it appears that the model fit tends to overpredict the SFR and luminosity relative to the 70 micron flux estimate. 


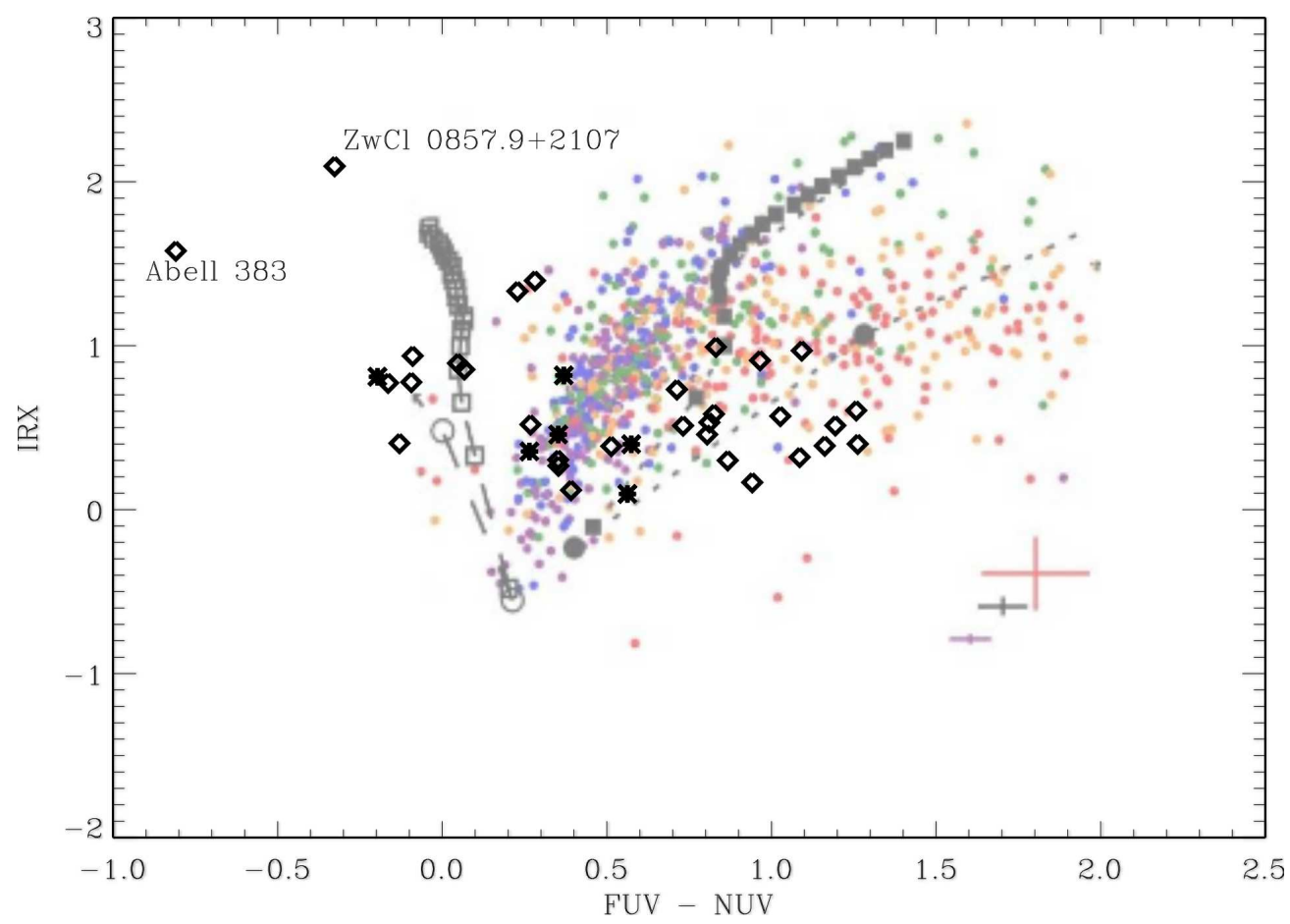

Fig. 13.- IR excess and UV color. The IR excess (IRX) is defined in Johnson et al. (2007a) to be the ratio of IR to UV luminosity. Objects from the cool core sample of Hicks et al. (2010) are marked with X's. Figure 6a from Johnson et al. (2007a) is plotted in the background on this graph. 


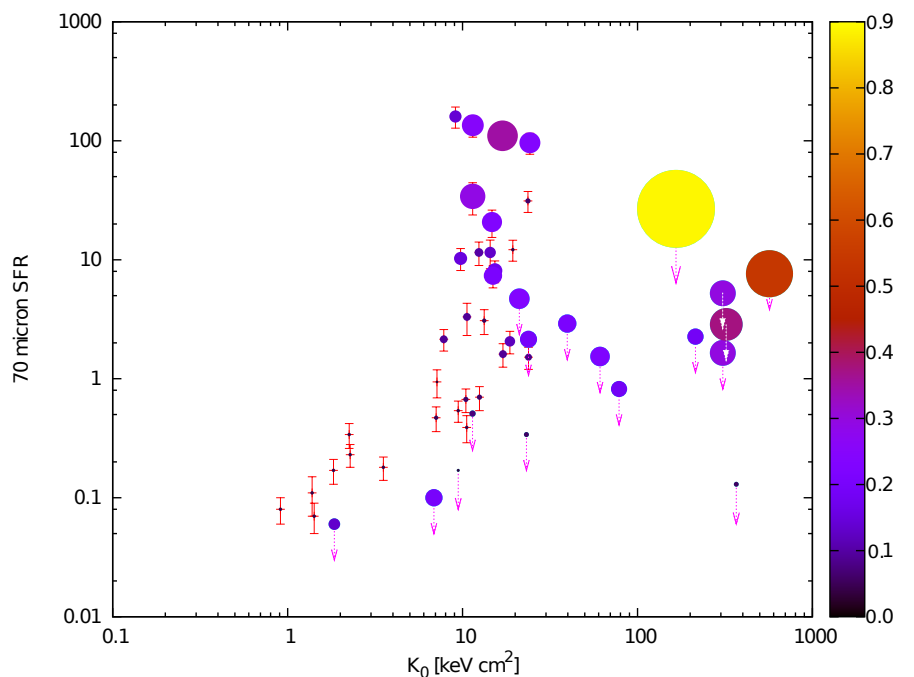

Fig. 14.- Relation between 70 micron SFR and central entropy. The objects are color coded and sized based on redshift (i.e. higher redshift, larger size). The color code ranges from redshift of 0.0 to 0.9. The "trend" that is seen in the lowest central entropy clusters is not a physical trend, but it is the same selection effect noted in Figure 4. Note that all BCGs in clusters with $K_{0}>30 \mathrm{keV} \mathrm{cm}{ }^{2}$ in this plot have only upper limits. 